Universidad Politécnica de Madrid

Escuela Técnica Superior de Ingenieros de Telecomunicación

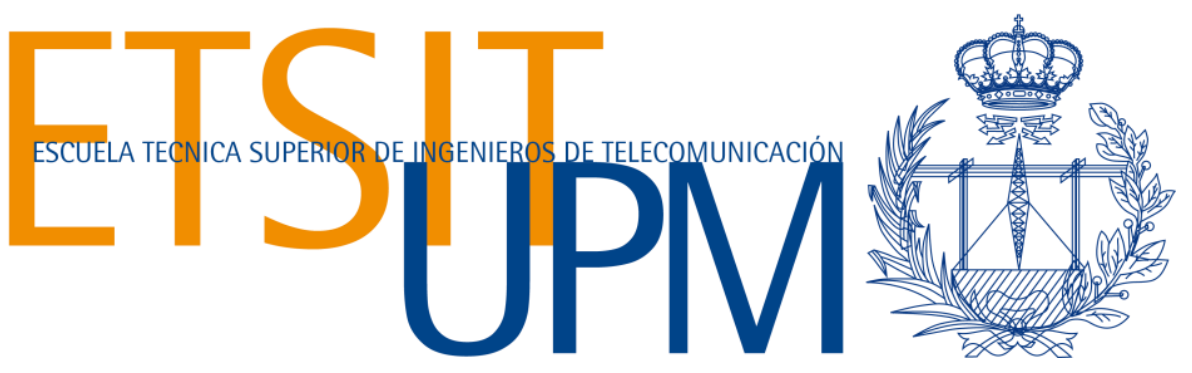

\title{
Optical imaging technologies and opto-biomechanical models for diagnostics and customized treatment in ophthalmology
}

Tesis doctoral

Andrés de la Hoz Durán

Ingeniero Biomédico 

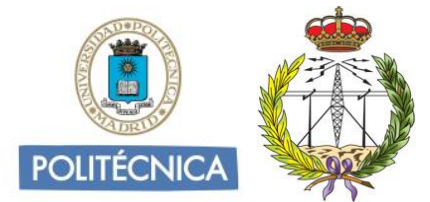

Departamento de Ingeniería Biomédica

Escuela Técnica Superior de Ingenieros de Telecomunicación

Universidad Politécnica de Madrid

\title{
Optical imaging technologies and opto-biomechanical models for diagnostics and customized treatment in ophthalmology
}

Tesis doctoral

\author{
Andrés de la Hoz Durán \\ Ingeniero Biomédico \\ Directores: \\ Susana Marcos Celestino \\ Dra. Ciencias Físicas \\ Francisco Javier Rojo Pérez \\ Dr. Ingeniero de Caminos, Canales y Puertos
}






\section{Table of Contents}
Abstract $\mathbf{v}$

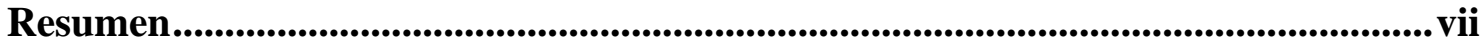

Acknowledgements ................................................................................................................................... ix

Index

1. Background ............................................................................................................ 1

1.1. Motivation ............................................................................................................................... 1

1.2. The Eye ........................................................................................................................................... 1

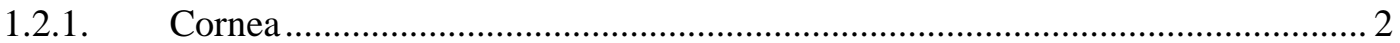

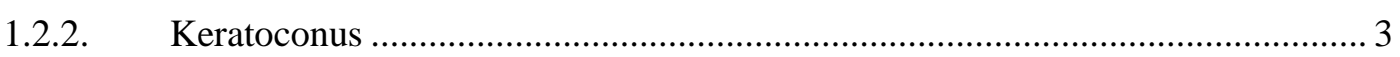

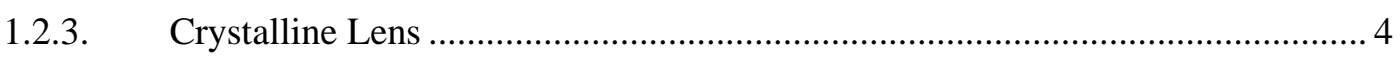

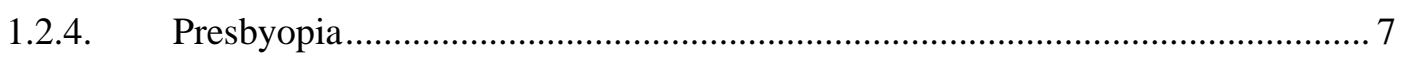

1.2.5. Proposed methods of presbyopia correction ................................................ 9

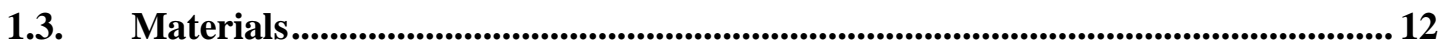

1.3.1. Intraocular Lens and Contact Lens Materials ................................................. 12

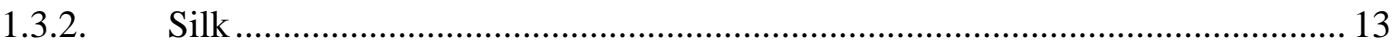

1.4. Optical Imaging Technologies ............................................................................................. 14

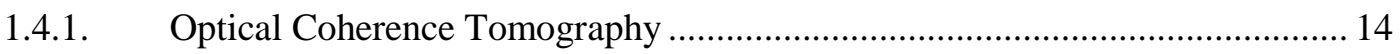

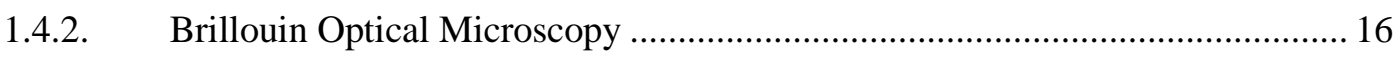

1.5. Continuum Mechanics............................................................................... 18

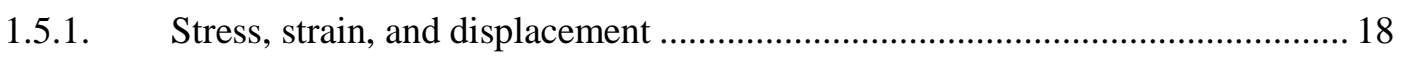

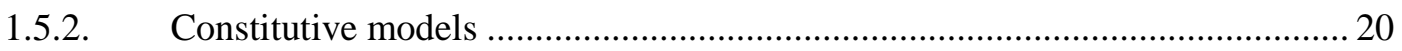

1.5.3. Fitting material data to constitutive models ..................................................... 24

1.6. The Finite Element Method ...................................................................................... 27

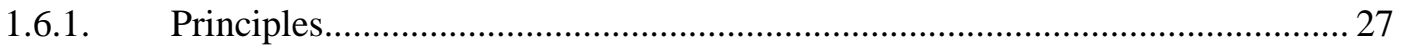

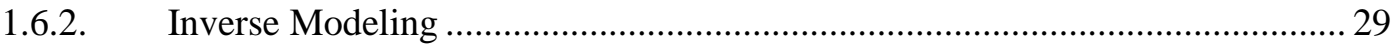

1.7. Hypothesis .................................................................................................................... 29

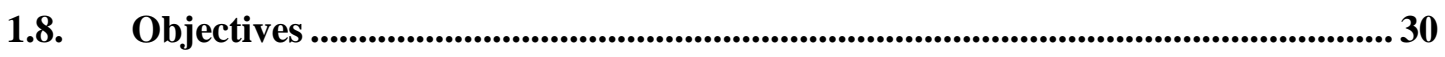

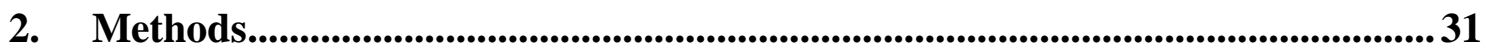

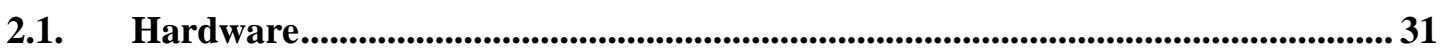

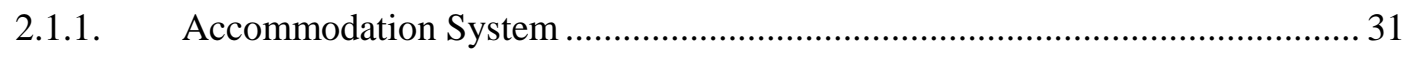

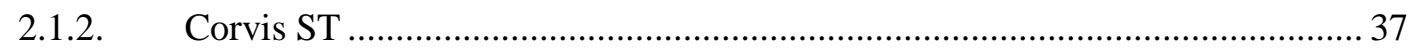

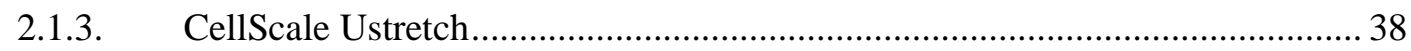




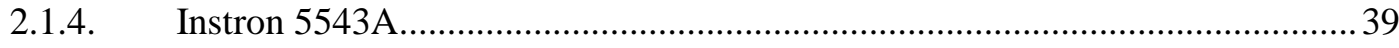

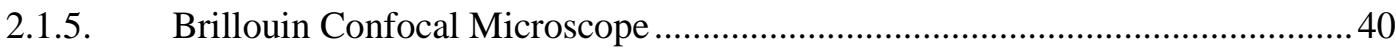

2.2. Software ..............................................................................................................................4 41

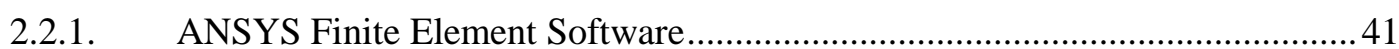

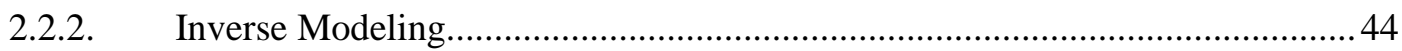

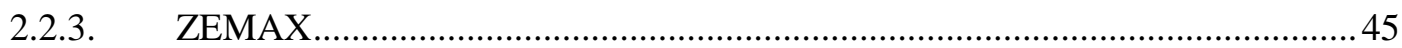

2.3. Applications of the technology in the thesis................................................................46

3. Material properties of the cornea from air puff deformation and numerical

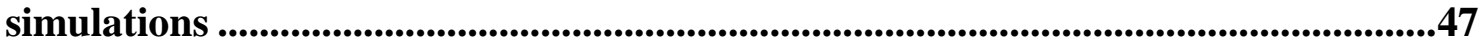

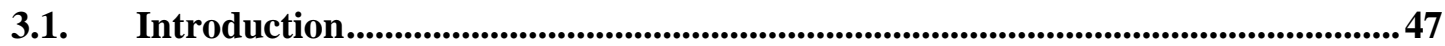

3.2. Protocols for corneal mechanical characterization................................................48

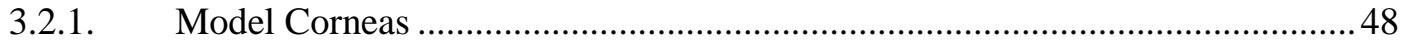

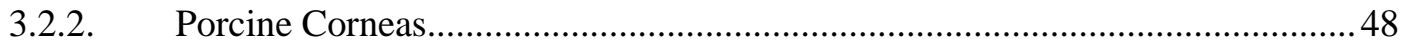

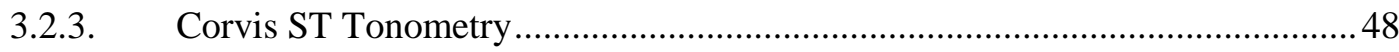

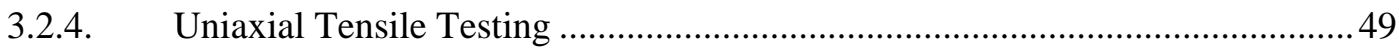

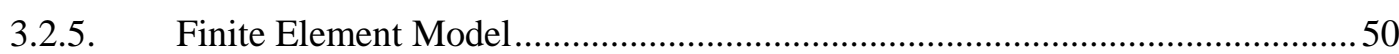

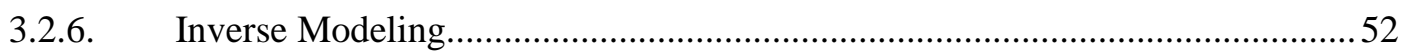

3.3. Mechanical behavior of corneas and model materials...........................................52

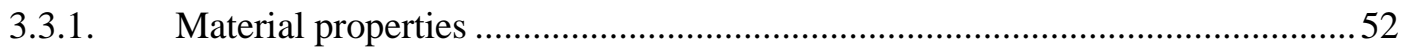

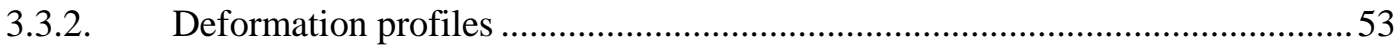

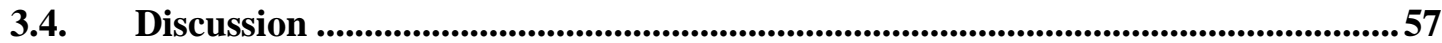

3.5. Conclusions ...............................................................................................................................58

4. Rose Bengal Photobonded Silk Fibroin Films for corneal wound healing ......59

4.1. Introduction.....................................................................................................................59

4.2. Protocols for silk film photobonding and evaluation.................................................60

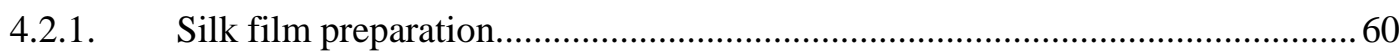

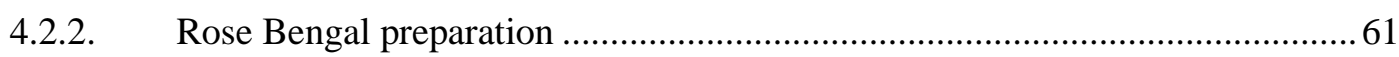

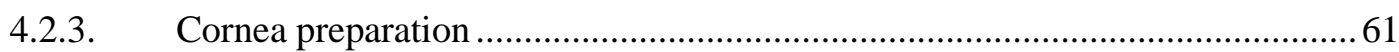

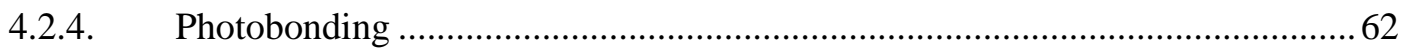

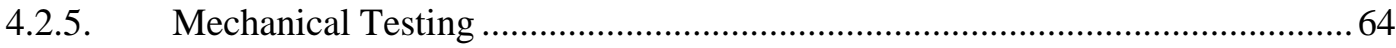

4.3. Mechanical performance of Rose Bengal photobonding .......................................66

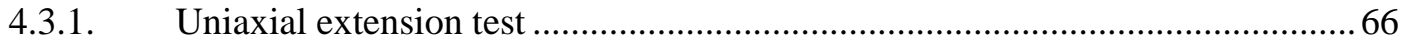

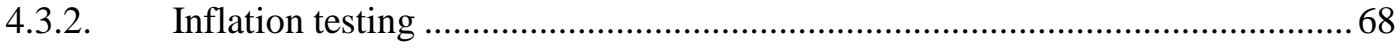

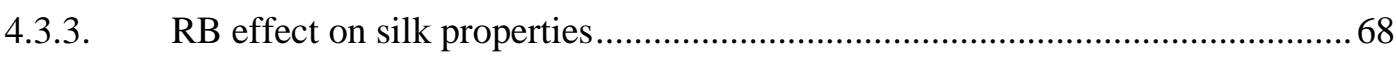




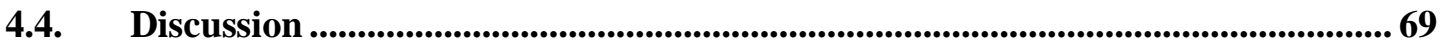

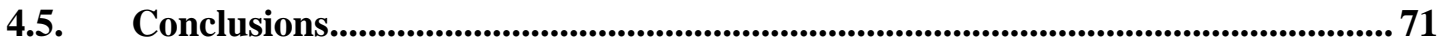

5. Material properties of the crystalline lens from Brillouin microscopy and

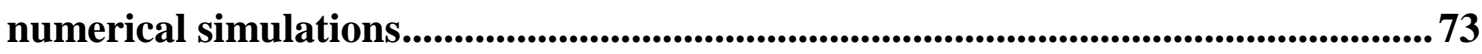

5.1. Introduction ...................................................................................................................... 73

5.2. Numerical analysis of the crystalline lens properties based on Brillouin microscopy measurements .................................................................................................................. 74

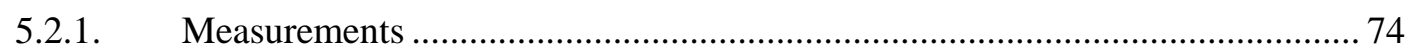

5.2.2. Finite Element Model of Lens Compression …….............................................. 75

5.2.3. Finite Element Model of Accommodation........................................................... 79

5.3. Crystalline lens properties and accommodation amplitude...................................... 82

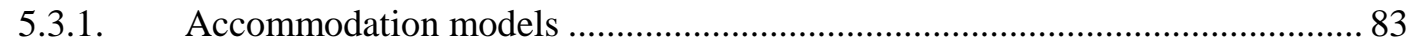

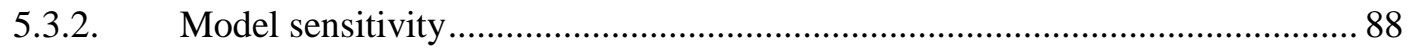

5.4. Limitations of Brillouin elasticity models for crystalline lenses ............................. 88

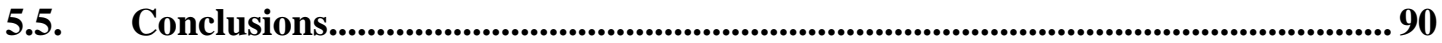

6. Design and ex situ performance of an accommodating intraocular lens..........91

6.1. Introduction .......................................................................................................................99

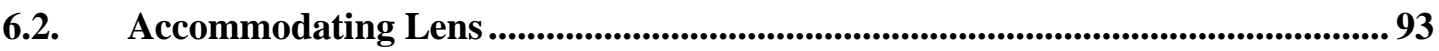

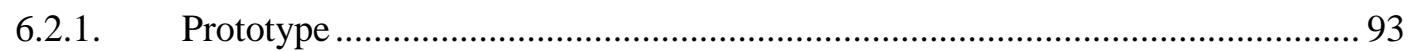

6.2.2. Material evaluation \& constitutive models .......................................................... 94

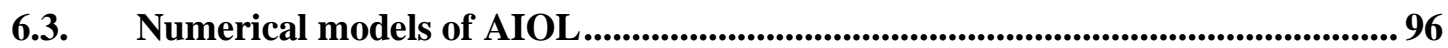

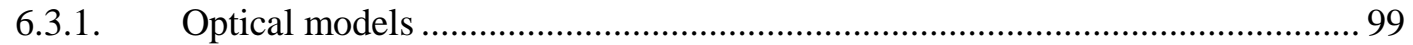

6.3.2. Experimental models for opto-mechanical evaluation..................................... 101

6.3.3. AIOL prototype evaluation: experimental protocols ...................................... 102

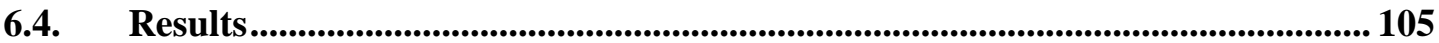

6.4.1. Material evaluation \& constitutive models .................................................... 105

6.4.2. Ex vivo optical changes upon accommodation ............................................. 107

6.4.3. Geometry changes upon accommodation ....................................................... 109

6.4.4. Comparison between LRT and OCT experiments ........................................ 113

6.4.5. Evaluation of material parameters in opto-mechanical in vivo model............. 114

6.4.6. Evaluation of geometric parameters in opto-mechanical in vivo model......... 116

6.4.7. AIOL higher order aberrations and their change with accommodation.......... 117

6.5. Discussion ........................................................................................................................... 118

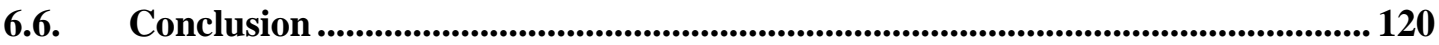

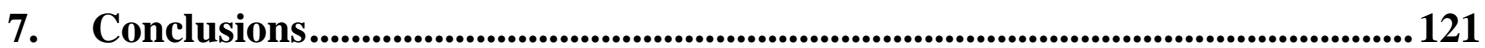




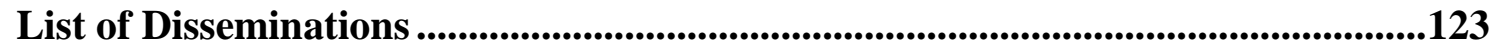

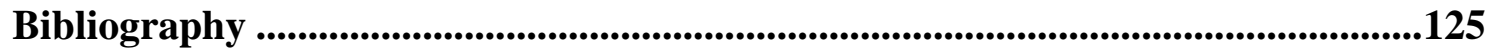




\section{Abstract}

This thesis presents an experimental work in which optical technologies and numerical and mechanical models are used to develop methods for diagnostics (through analysis of the mechanical properties of tissues) and treatment (through the development of techniques and devices) in ophthalmology, specifically on tissues of the anterior segment of the eye: the cornea and the crystalline lens.

Numerical models of crystalline lenses and corneas were developed. Experimental measurements of lenses and corneas under loading were taken, and an inverse modeling approach was used to combine the experimental data and numerical models to retrieve the mechanical parameters of the tissues. The results were validated in order to assess their reliability and evaluate the use of the models in diagnosing ocular conditions.

Numerical models were used to develop an accommodating intraocular lens. A custom experimental system for the evaluation of optical and geometric changes upon the lens under quantifiable loading were developed and validated. A prototype of the accommodating lens concept was manufactured and evaluated in the system, and the results were validated by numerical simulations.

Finally, the viability of a light-based technology, Rose Bengal photobonding, was evaluated for bonding of corneal tissue with silk fibroin films. Extensiometry and inflation measurements were done in order to evaluate the strength of the bonding force between tissue and silk. 


\section{Resumen}

Esta tesis presenta un trabajo de investigación en el que se emplearon tecnologías ópticas y mecánicas, así como modelos numéricos para desarrollar métodos de diagnóstico (mediante el análisis de las propiedades mecánicas de tejidos) y tratamiento (mediante el desarrollo de técnicas y dispositivos) en oftalmología, específicamente en tejidos del segmento anterior: la córnea y el cristalino.

Se desarrollaron modelos numéricos del cristalino y la córnea. Se hizo una serie de medidas experimentales de cristalinos y córneas bajo diferentes condiciones de carga. Mediante la técnica de modelado inverso se combinaron los datos experimentales y los modelos numéricos para obtener las propiedades mecánicas inherentes de los tejidos. Los resultados fueron validados y se evaluó su fiabilidad.

Se desarrollaron modelos numéricos para el diseño de una lente acomodativa. Se desarrolló y validó un sistema de evaluación experimental de los cambios de propiedades ópticas y mecánicas en acomodación. Se fabricó un prototipo de lente acomodativa que fue evaluado en este sistema, y los resultados se validaron mediante la plataforma de simulación numérica.

Se ha estudiado la viabilidad de una técnica óptica, fotoenlace químico con Rosa de Bengala, para el uso de películas de fibroína de seda sobre el tejido corneal. Se hicieron ensayos de tracción y de inflado, para evaluar la resistencia de la unión entre tejido y seda. 


\section{Acknowledgements}

I would like to thank the co-directors of this thesis, Drs. Susana Marcos and Francisco Rojo, for their steady guidance, patience, and wisdom. To Susana, thank you for giving me the opportunity to work at the Visual Optics and Biophotonics Group and the trust you have placed on me. I would also like to thank my past and present colleagues from the Visual Optics and Biophotonics Group for their contribution, assistance, and support during the period of my predoctoral research, as well as Dr. Fiorenzo Omenetto and the SilkLab group at Tufts University for welcoming me to their lab for a short-term research stay. And to Cassandra, thanks for your support, encouragement, and patience. This thesis could not have been done without all of your help and support. 



\section{Index}

\section{Keywords}

ACD: Anterior chamber depth

AIOL: Accommodating intraocular lens

AL: Axial length

CPR: Central-peripheral deformation ratio

CT: Corneal thickness

CXL: UV-corneal collagen cross-linking

D: Diopters

DA: Deformation Amplitude

EPP: Equatorial Plane Position

GRIN: Gradient Refractive Index

IOL: Intraocular lens

IOP: Intraocular pressure

LASIK: Laser-assisted in situ keratomileusis

LRT: Laser ray tracer/ing

MC: Model cornea

OCT: Optical coherence tomography

PALS: Progressive addition lenses

PC: Porcine corneas

RBCXL: Rose Bengal cross-linking

RBPh: Rose Bengal photobonding

\section{Tables}

Table 3.1: Nominal properties of model corneas................................................... 48

Table 4.1: Summary of experimental conditions for SF strips. ...................................63

Table 5.1: Geometry data for crystalline lenses of 29 and 45 years, from Burd 2002... 79

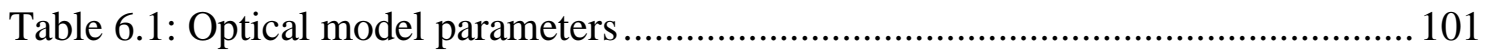

Table 6.2: Maximum stress and strain for various constitutive models. ....................... 106

Table 6.3: Neo-Hookean coefficient value for all evaluated materials ........................ 107 


\section{Figures}

Figure 1.1: Anterior segment of the eye. (Ortiz, 2012) ................................................2

Figure 1.2: The layers of the cornea. (The Discovery Eye Foundation) ...........................3

Figure 1.3: Corneal crosslinking. ( Keratoconus Australia Inc.) ....................................

Figure 1.4: Differences between accommodated and relaxed or disaccommodated state

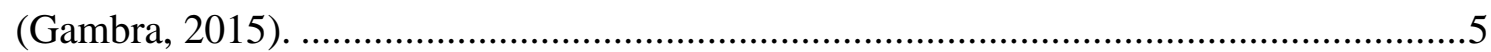

Figure 1.5: Representation of crystalline lens, divided into embryonic nucleus (en), fetal nucleus (fn), juvenile nucleus (jn), adult nucleus (an), and cortex. (Taylor et al., 1996) .6 Figure 1.6: Reconstruction of gradient refractive index of the crystalline lens (de Castro

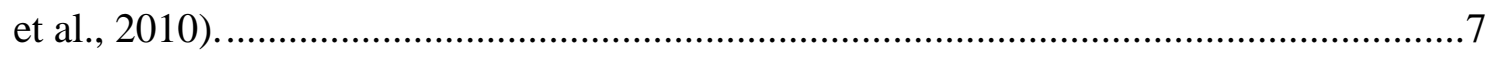

Figure 1.7: A) Crystalens. (Jorge L. Alió et al., 2017). B) FluidVision. (Alcon) ............11

Figure 1.8: Different types of materials and structures derived from bombyx mori silk

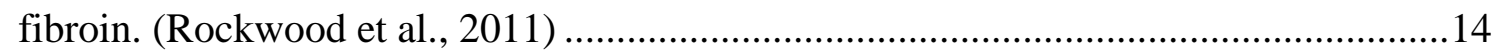

Figure 1.9: Anterior segment reconstruction with OCT (Ortiz et al., 2013)...................15

Figure 1.10: Brillouin frequency shift in cornea (Scarcelli et al., 2012).......................17

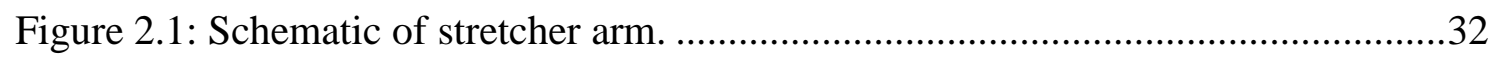

Figure 2.2. Schematic view of stretcher device from above. ......................................33

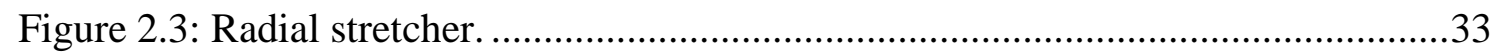

Figure 2.4. A) Schematic of Laser Ray Tracing system. B) Photograph of system. ......34

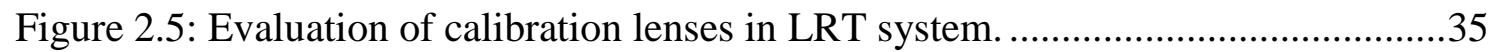

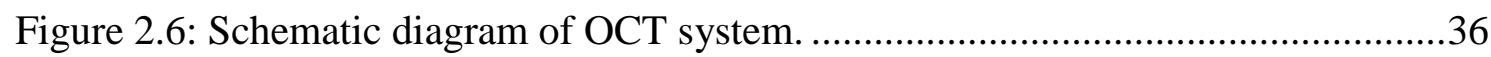

Figure 2.7: OCT component of the accommodation system: reference arm and

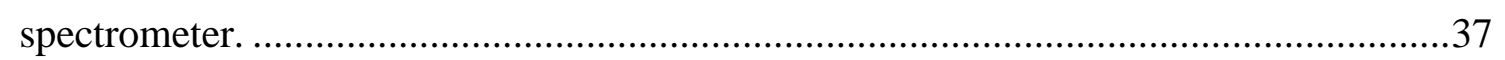

Figure 2.8: Top view of calibration grid retrieved from OCT measurements for fan

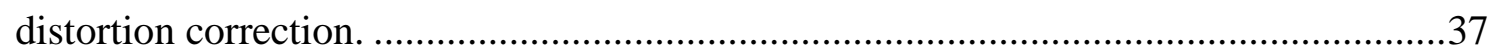

Figure 2.9: Ustretch system used for the work in this thesis......................................39

Figure 2.10: Instron 5543A. Clamps are within custom-built immersion bath...............40

Figure 2.11: ANSYS Workbench DesignModeler geometry of a 2D axisymmetric

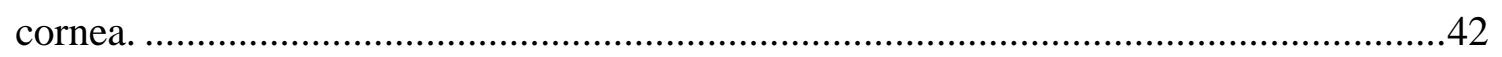

Figure 2.12: Meshing in A) ANSYS Mechanical APDL B) ANSYS Workbench. ........42

Figure 2.13: Element options for PLANE183 ….....................................................43 
Figure 2.14: Boundary conditions and loads in A) Mechanical APDL B) ANSYS

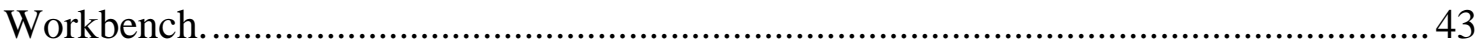

Figure 2.15: Displacement loads in A) Mechanical APDL B) Ansys Workbench. ....... 44

Figure 2.16: Schematic of inverse modeling procedures............................................ 45

Figure 3.1: Schematic of experimental set-up ......................................................... 49

Figure 3.2: Finite element model of MC under loading ............................................ 51

Figure 3.3: A) Material coefficients $\left(\mathrm{C}_{10}, \mathrm{C}_{01}\right)$ retrieved from inverse modelling process.

B) Equivalent Young's Modulus of materials at lower strain rates. $G=G e n t l e, S=$ Saphir,

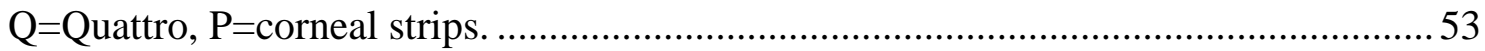

Figure 3.4: Reconstructed uniaxial response vs. experimental uniaxial response for

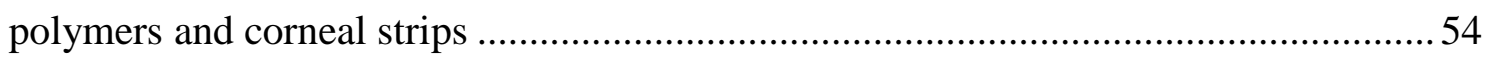

Figure 3.5: Deformation parameters for all corneas tested......................................... 55

Figure 3.6: Spatial and temporal profiles of MC and PC deformation.......................... 56

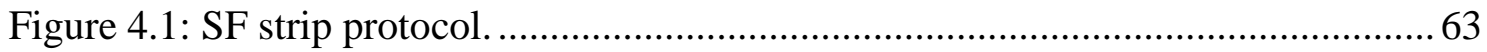

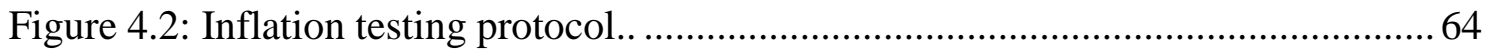

Figure 4.3: SF strip and corneal strip pulled apart in uniaxial stretcher.......................65

Figure 4.4: Inflation testing. Ocular globe is held inside container; syringe enters the

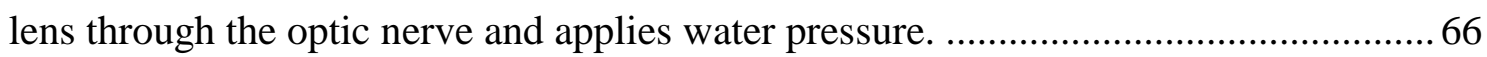

Figure 4.5: Maximum force from extensiometry testing as a function of fluence .........67 67

Figure 4.6: Comparison between RB-stained, rhodamine-stained, and unstained SF

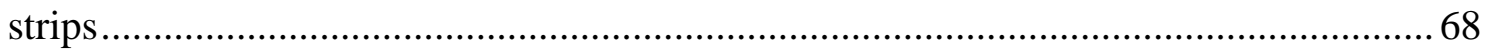

Figure 4.7: Maximum pressure recorded for RB-stained SF discs bonded onto cornea.

The effect of increasing fluence is reflected in the data. 69

Figure 4.8: Maximum stretch and stress values in RB-stained and control films.

Figure 5.1: A) Brillouin frequency shift map of the same lens (GHz). B) Compression

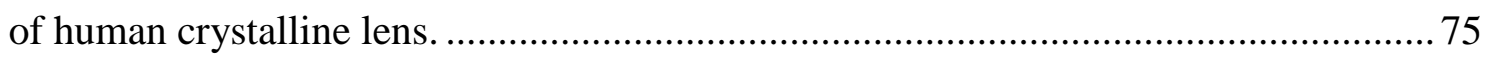

Figure 5.2: Finite element model of lens compression............................................. 76

Figure 5.3: Finite element model of accommodation for 29-year-old lens .................... 80

Figure 5.4: Lens compression model after solving. Von Mises strain. .......................... 82

Figure 5.5: Simulated and experimental compression results from 14 and 37 year old lenses.

Figure 5.6: Accommodative amplitude of 14-year-old elasticity maps and 29-year-old data from Burd. Boundary condition: displacement. 
Figure 5.7: Accommodative amplitude of 37-year-old elasticity maps and 29-year-old data from Burd .85

Figure 5.8: Accommodative amplitude of 14-year-old elasticity maps and 29-year-old data from Burd..

Figure 5.9: Comparison between deformed surfaces of Brillouin and Burd material

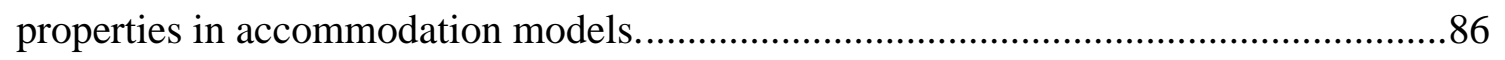

Figure 5.10: Evaluation of elasticity models with varying gradient coefficient k. .........86

Figure 5.11: Change in accommodative amplitude for reduced elasticity maps. ...........87

Figure 5.12: Finite element solution of accommodation model. ....................................87

Figure 6.1: AIOL concept. A) cross-sectional view. B) 3D isometric view..................93

Figure 6.2: AIOL prototype. .94

Figure 6.3: A) Finite element model of AIOL prototype. B) Displacement of AIOL prototype in model (units: $\mathrm{mm}$ ). C) ZEMAX model of lens in accommodation system's

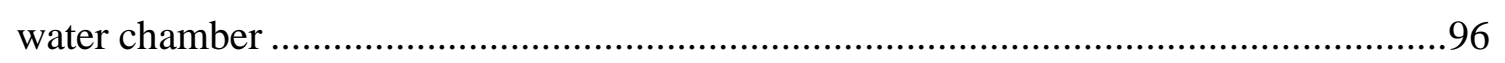

Figure 6.4: Finite element model of AIOL for in vivo performance. ............................97

Figure 6.5. Calculated curvature and thickness values and variation with element size 98 Figure 6.6: Schematic of optical model of eye and AIOL .........................................101

Figure 6.7: A) Radial stretcher system. B) Prototype on stretcher. ...............................102

Figure 6.8: Schematic of lens evaluation in accommodation system. ..........................103

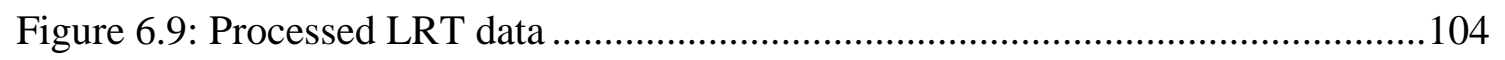

Figure 6.10: Stress-stretch ratio relationship for Polymacon material...........................106

Figure 6.11: Representative stress-stretch ratio curves for all materials evaluated ......106

Figure 6.12: Representative trial in LRT system. ................................................... 108

Figure 6.13: Full results of LRT experiments for 4 and $4.5 \mathrm{~mm}$ spots. ........................109

Figure 6.14. Results from OCT experiments and analysis. A) Anterior surface radius B)

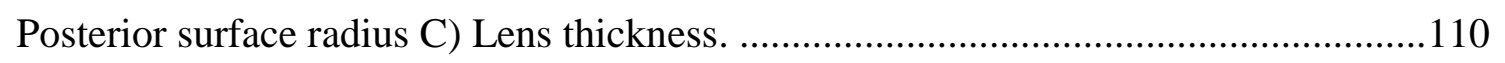

Figure 6.15: Reconstruction of OCT data. A) AIOL reconstruction with cross-sectional image of B-scan. B) Reconstruction of full volume in accommodated (right) and disacommodated (left) states. C) Top view of reconstruction... ..................................111

Figure 6.16. Comparison between spherical and conic reconstruction of lens surfaces.

Figure 6.17. Power calculations using OCT surface data. .114 
Figure 6.18: Diopter change calculated for all evaluated constitutive models.

Figure 6.19: Relationship between refractive index and equivalent Young's Modulus. 116

Figure 6.20: Evaluation of accommodative amplitude for varying mechanical and optical properties.

Figure 6.21: Effect of varying anterior and posterior radii of curvature on accommodative amplitude in finite element model.

Figure 6.22. Surface maps of disaccommodated AIOL from simulation. A) Anterior surface, B) Posterior surface. 118 



\section{Background}

\subsection{Motivation}

The two predominant structures of the eye's anterior segment are the cornea and the crystalline lens. Both are highly structured, organized tissues, and their response to loading is relevant to their functionality. The cornea and the crystalline lens can be affected by pathologies such as keratoconus or presbyopia, or conditions such as penetrative injuries, which negatively affect their structure and function. As such, there is both a need to assess these tissues' mechanical properties and behavior, and to develop effective treatments for these pathologies or conditions.

Optical imaging technologies are suitable to ophthalmology, due to the anterior segment structures' transparency, and as such, have been used for imaging and investigation of the cornea and crystalline lens. Additionally, optics-based technologies have been used to treat the cornea and crystalline lens' condition, in the form of approaches such as LASIK and crosslinking.

Mechanical testing of the anterior segment's structures is a complex endeavor. The application or measurement of loads and responses in vivo is not easily resolved into a simple analytical form, preventing the conversion of experimental data into more relevant parameters, such as a material modulus of elasticity. A method of bridging this gap is the use of numerical models, which can model behavior under loading. If targets of expected behavior are known or observed, the relevant parameters could be reconstructed from the model.

A better understanding of how optical technologies and mechanical/optical models can be used to retrieve material parameters and predict behavior under loading will help in designing and evaluating treatment for ocular pathologies.

\subsection{The Eye}

The eye is the organ of the visual system that receives and processes light. Light is refracted through the anterior segment, which includes the cornea and the crystalline lens, two lenses that have different structural and optical functions. The anterior segment also includes the iris, a pupil that modulates the amount of light entering the 
lens, and the ciliary body, a radial muscle that exerts forces upon the crystalline lens. The anterior segment is filled with aqueous humor, a transparent, watery fluid. Figure 1.1 shows a representation of the anterior segment.

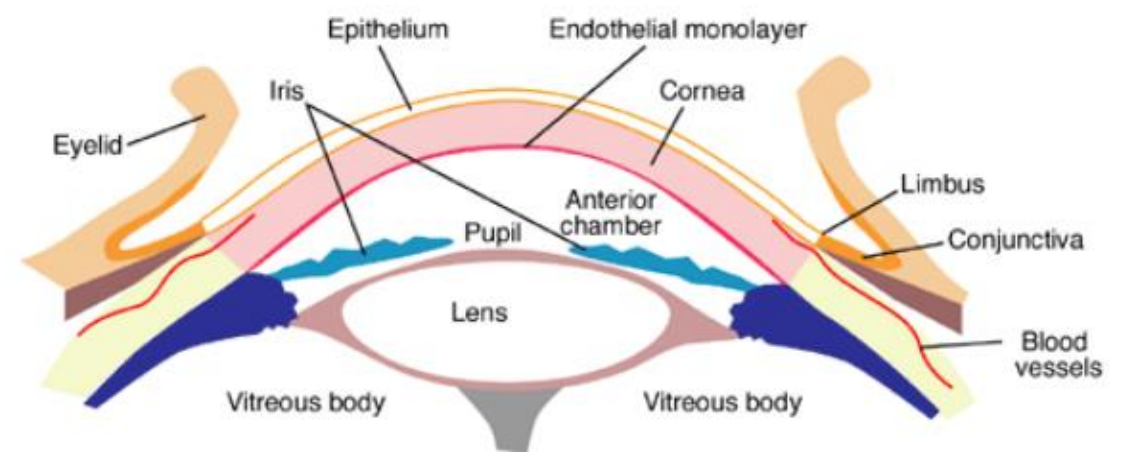

Figure 1.1: Anterior segment of the eye. (Ortiz, 2012)

\subsubsection{Cornea}

The cornea is the first refractive surface of the anterior segment and accounts for $2 / 3 \mathrm{rds}$ of the eye's refractive power. It is a thin, fixed-focus, meniscus-shaped lens of positive power and variable thickness, from $0.5 \mathrm{~mm}$ in the center to $0.65 \mathrm{~mm}$ in the periphery. The cornea is an avascular tissue composed of highly organized and spaced collagen fibrils, factors which contribute to its transparency (Qazi et al., 2010). Structurally it can be classified in 5 distinct regions or layers: the epithelium, Bowman's membrane, stroma, Descemet's membrane, and the endothelium, with the stroma comprising the majority of the corneal volume (represented in Figure 1.2). Its highly organized structure confers stiffness.

A mechanical parameter often used to describe corneal stiffness is the Young's Modulus, defined as the slope of the linear region of the material's stress-strain curve. Reports of the cornea's Young's Modulus (generally measured post-mortem) vary by close to 2 orders of magnitude, with values ranging from 0.2 to $3 \mathrm{MPa}$ as measured by uniaxial (Wollensak et al., 2003) and inflation testing (Elsheikh et al., 2007). As a result of its high fluid content (78\%), the cornea also exhibits viscoelastic properties. A number of pathologies can affect the cornea's structure and its ability to resist deformation, such as diabetes (Kaji et al., 2000) and keratoconus. 


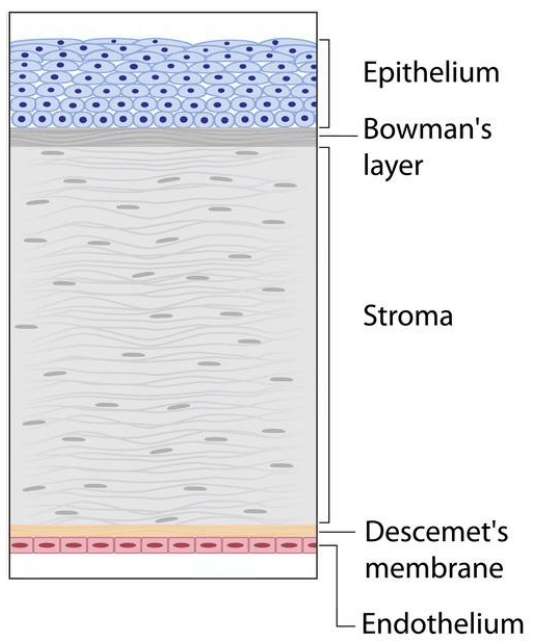

Figure 1.2: The layers of the cornea. (The Discovery Eye Foundation)

\subsubsection{Keratoconus}

Keratoconus is corneal pathology involving a regional loss of collagen fibril orientation resulting in thinning and weakening of the relevant area of the cornea, and excessive bulge under loading from pressure exerted by the aqueous humor (Vellara \& Patel, 2015). The bulge resembles a cone (hence the name) and causes an increase in the optical aberrations of the eye. Standard treatment for keratoconus was, until recent years, limited to corneal transplants, or the implantation of intrastromal rings made of PMMA to reshape the cornea.

Collagen cross-linking has been proposed as a way to treat the progression of keratoconus. This technique consists of dyeing or staining the cornea with a photosensitizer, and then irradiating with light of a specific wavelength. The combination of photosensitizer and irradiation induces the generation of cross-links between the collagen fibers, which increases the stiffness of the tissue (represented in Figure 1.3). The most common crosslinking technique is UV-Corneal Collagen Crosslinking (CXL), which uses riboflavin as photosensitizer and ultraviolet light.

Another type of crosslinking that has been proposed is Rose Bengal Crosslinking (RBCXL). This technique uses Rose Bengal sodium salt (RB) and green light (532 nm). This method is of interest because its duration is considerably shorter: CXL requires 30 minutes of irradiation, whereas RBCXL requires 10. An additional advantage is that diffusion of RB into the tissue is limited to $100 \mu \mathrm{m}$, therefore protecting the endothelial cells from photodamage. 


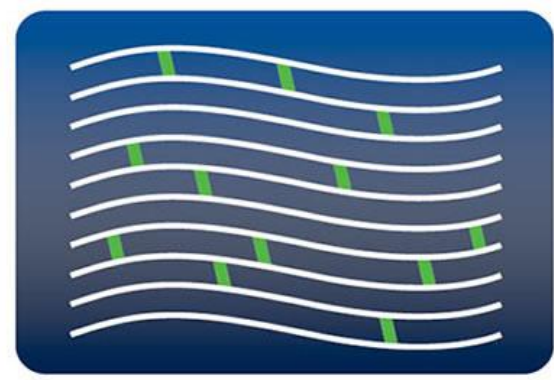

before CXL: weaker bonds in collagen

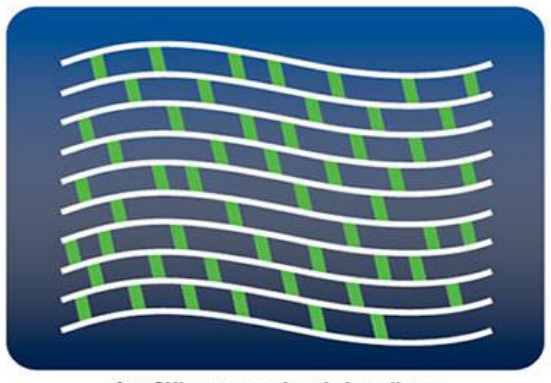

after CXL: stronger bonds in collagen

Figure 1.3: Corneal crosslinking. ( Keratoconus Australia Inc.)

\subsubsection{Crystalline Lens}

The crystalline lens accounts for the rest of the eye's refractive power, as well as giving the human visual system its capability to focus on objects at different distances. It is located behind the cornea and iris, in front of the vitreous body of the eye. The average central thickness of the crystalline lens is approximately $4 \mathrm{~mm}$, and the average diameter is $9 \mathrm{~mm}$ (Martinez-Enriquez et al., 2016).

\section{Accommodation}

The crystalline lens is connected to the ciliary muscle, a smooth muscle ring, through thin fibers called zonules, which extend to the lens' equator. The crystalline lens is responsible for the process of accommodation, which is the ability of the eye to change its focusing power. The most widely accepted theory of accommodation is Helmholtz's. Accommodation occurs when the ciliary muscle relaxes, the diameter increases, and the lens surfaces adopt their maximally curved shape. Conversely, when equatorial forces are applied to the crystalline lens through the zonules, the lens surfaces flatten, the equatorial diameter increases, and central thickness decreases. The geometrical changes in the lens result in changes in the optical power (Charman, 2008). Thus, far vision is associated with the relaxed state of the ciliary muscle, and near vision is associated with its contracted or accommodated state. Figure 1.4 shows a representation of the changes in the ciliary muscle, crystalline lens, and zonules observed during the accommodation process.

\section{Physiology}

The crystalline lens' regions have different mechanical and structural properties. The lens capsule is a relatively stiff membrane of approximately $13 \mu \mathrm{m}$ in thickness, and acts as a shell for the internal contents of the crystalline lens. The contents of the lens 
inside the capsule are generally classified into two distinct regions, the lens cortex and the lens nucleus. These regions depend on lens fiber cells, which are the main cells of the crystalline lens. These are highly elongated cells, lacking organelles and nuclei, and containing fiber-specific proteins (Kuwabara, 1975). They derive from epithelial cells and undergo a differentiation process, one that results in the lens growing and adding fibrous layers. The lens cortex consists of fiber cells undergoing differentiation. The lens nucleus consists of fully differentiated fiber cells, and has distinct sub-regions (the adult nucleus, juvenile nucleus, fetal nucleus, and embryonic nucleus) corresponding to the period in the organism's lifespan in which the fibers developed (Lim et al., 2009). Figure 1.5 shows a representation of the different layers of the lens.

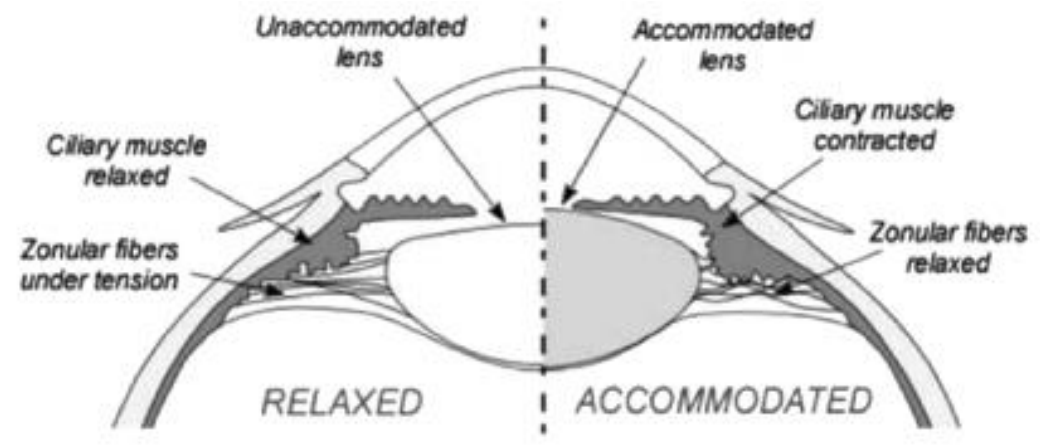

Figure 1.4: Differences between accommodated and relaxed or disaccommodated state (Gambra, 2015).

The physiological differences in the lens' internal structure are also observed in its mechanical properties. The lens nucleus and cortex have been measured to have distinct responses to loading. Mechanical testing such as indentation tests (Weeber et al., 2007) and spinning tests (Wilde et al., 2012) have found variations in lens stiffness across the two regions, with the cortex having higher stiffness than the nucleus. Non-invasive techniques such as Brillouin microscopy have found higher stiffness values in the nucleus than in the cortex (Besner et al., 2016). Imaging of the lens during accommodation shows thickness changes concentrating on the nucleus while central cortical thickness remains constant (Dubbelman et al., 2003), suggesting that it is the nucleus that deforms during loading, on account of having less stiffness than the cortex.

The lens fibers contain proteins called crystallins, which account for the high refractive index of the crystalline lens. These proteins account for a third of the lens' wet weight and $90 \%$ of the lens' dry weight (Michael \& Bron, 2011). The three types of crystallins present in the lens are $\alpha-, \beta$-, and $\gamma$-crystallins. $\alpha$-crystallins have structural and 
chaperone functions, whereas $\beta$ - and $\gamma$-crystallins are thought to be primarily structural. $\gamma$-crystallins are present at higher concentration in the nucleus of the lens and largely produced prenatally; refractive index increments are also higher for $\gamma$-crystallins (Zhao et al., 2011). The overall protein distribution in the lens varies spatially, with values of approximately $0.22 \mathrm{~g} / \mathrm{cm}^{3}$ in the cortical region and $0.45 \mathrm{~g} / \mathrm{cm}^{3}$ in the nuclear region, with variation in the cortical zone and relatively flat in the nucleus (Fagerholm et al., 1981).

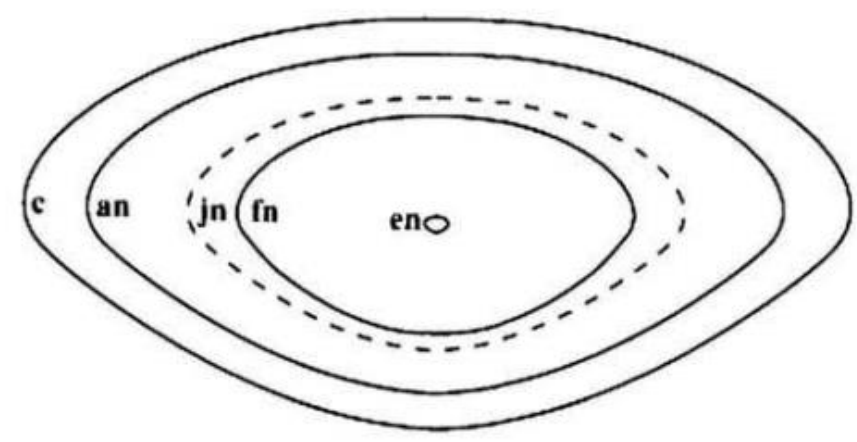

Figure 1.5: Representation of crystalline lens, divided into embryonic nucleus (en), fetal nucleus (fn), juvenile nucleus (jn), adult nucleus (an), and cortex. (Taylor et al., 1996)

The organization of the proteins within the fiber cells, the fiber cells' tightly packed and organized structure, and the lack of light-scattering organelles in the fibers are responsible for the lens' transparency (Bassnett et al., 2011). The distribution of proteins and the organization of the lens fibers spatially vary through the lens. This is due to the process of lens growth being a product of addition of new fibers to the lens surface. The more central fibers are fully differentiated, metabolically inactive, and denser. The newer fibers along the cortex are still undergoing differentiation. These changes in density and protein distribution mean that the crystalline lens is not optically homogenous; the refractive index decreases the closer we are to the periphery of the lens. This positive lens gradient (represented in Figure 1.6) results in an increased equivalent refractive index for the overall lens, and higher power overall (Charman $\&$ Atchison, 2014; de Castro et al., 2010; Jones et al., 2005). The lens' gradient refractive index, or GRIN, is also responsible for the negative spherical aberration of the crystalline lens. Flattening of the GRIN profile with aging is responsible for the shift of the spherical aberration towards positive values with age (Birkenfeld et al., 2013; Sheil \& Goncharov, 2017). 


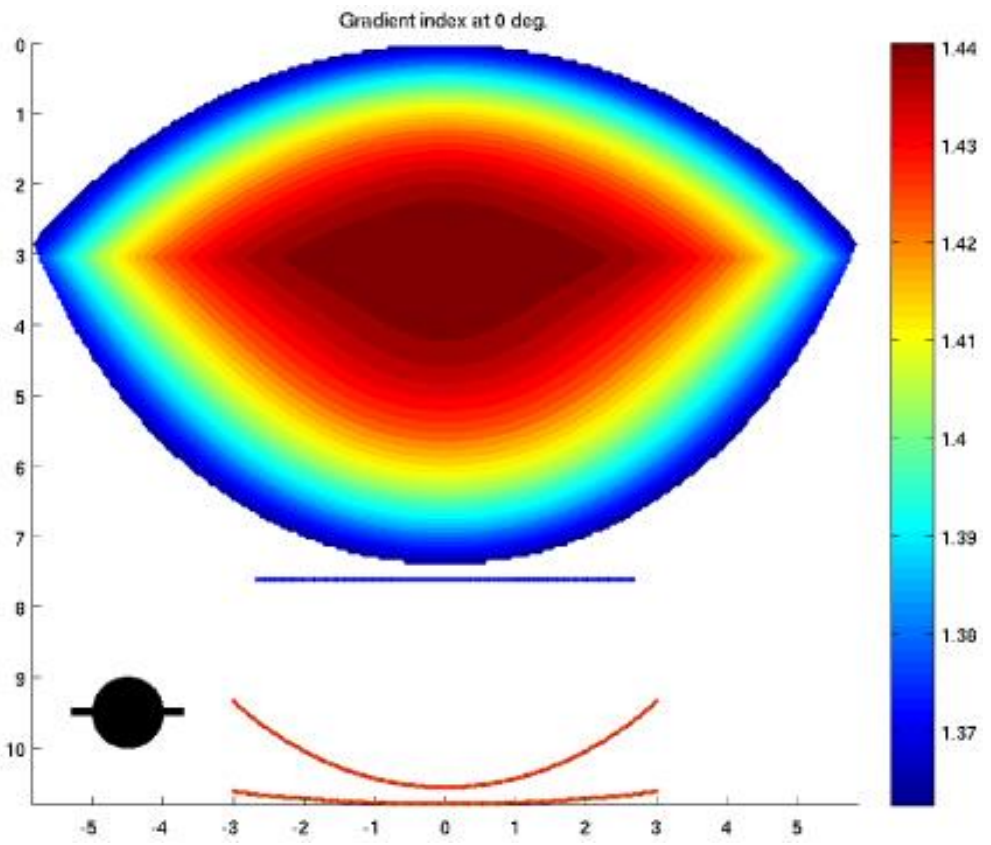

Figure 1.6: Reconstruction of gradient refractive index of the crystalline lens (de Castro et al., 2010).

\subsubsection{Presbyopia}

Presbyopia is the age-related loss of the eye's accommodative ability. Different theories have been proposed to explain this loss of accommodation, focusing on the different elements of the accommodative apparatus. The ciliary muscle has been shown to maintain its capability to contract and expand with aging (Tabernero et al., 2016), even though there are some age-related changes to its overall physiology (Croft et al., 2013). The source of presbyopia is believed to be the changes in the crystalline lens with aging, especially its stiffness.

A number of changes occur in the crystalline lens with aging. Its equatorial diameter and thickness increase with age (Kasthurirangan et al., 2011), as a result of additional fiber layers being added onto its periphery. The mechanical properties of the lens are also believed to change with aging, although the magnitude of the reported changes varies depending on the method used to measure. Spinning tests done by Fisher and improved upon by Wilde (Wilde et al., 2012) found that both cortex and nucleus harden with age, with the nucleus hardening at a quicker rate and becoming stiffer than the cortex at around age 45. Indentation testing done by Weeber (Weeber et al., 2007) found a similar trend of increasing stiffness in both nucleus and cortex at different rates, and a trend towards a negative gradient with age, matching closely with the onset of presbyopia. Other methods, such as Brillouin microscopy, have found higher stiffness 
values for the nucleus than the cortex, constant throughout age, and variations in the gradient profile, which steepens over time (Bailey et al., 2010; Scarcelli et al., 2011). In vitro stretching tests on rhesus monkey lenses performed by Glasser also found that the lens' increased stiffness after aging was responsible for the inability to accommodate (Glasser \& Wendt, 2013).

Changes are observed in the protein distribution and composition within the lens with aging. Proteins do not diffuse between regions of the lens, which indicates that the lens' central proteins are its oldest. The older proteins of the lens, present in its nucleus, unfold and denature with age. There is evidence that $\alpha$-crystallins are responsible for suppressing aggregation of these proteins (Rao et al., 1995). However, the proportion of water soluble, low molecular weight, to water insoluble, high molecular weight proteins changes with age, particularly in the nucleus, where the water soluble, low molecular weight $\alpha$-crystallin disappears after age 40 (McFall-Ngai et al., 1985). A study of these high molecular weight proteins found substantial differences in hardness of $\alpha$-crystallin pellets with different molecular weight, suggesting that there could be a relationship between the aggregation of crystallins into high molecular, water insoluble forms, and the lens' overall stiffness (Siezen \& Owen, 1983). Crystallin fragments have also been found to increase their presence in the lens' fibers with age (Santhoshkumar et al., 2008). These changes can also cause effects in lens transparency and light scattering, eventually taking on the form of a cataract.

Optically, the crystalline lens of the eye also presents changes with aging, as indicated above. A study using OCT to reconstruct the GRIN of lenses of varying ages found that while the central and surface refractive index values remained constant with age, the power coefficient of the model increased with age, resulting in a GRIN dominated by the central values for older lenses, a higher average refractive index, and a lower equivalent refractive index (de Castro et al., 2011). Estimations of the GRIN profile using MRI, assuming that index is proportional to water concentration, also reveal a parabolic profile with age that shifts to a plateau with age (Jones et al., 2005). Computational models of the optical variations in the lens with age are consistent with the observed trends in GRIN (Charman \& Atchison, 2014). These trends parallel the growth patterns of the crystalline lens, though no apparent relationship can be seen between the GRIN changes and the stiffness changes. 


\subsubsection{Proposed methods of presbyopia correction}

A number of approaches to treating presbyopia exist. The most common and successful approach is spectacles of positive power. These allow presbyopes to focus at near distance when wearing them, and at far distance when they are removed. Alternatively, progressive addition lenses (PALS), allow patients to see at far through the upper part of the spectacle, and progressively closer when they look down. Other increasingly prescribed alternatives involve surgical treatments (i.e. corneal surgery or intraocular lens implantation) or contact lenses.

\section{Static Corrections for presbyopia}

Most presbyopic solutions are static, aiming at providing the patient with a focus at far and a focus at near, or expanding the eye's depth of focus, but not attempting to restore dynamic accommodation. This approach includes monovision and simultaneous vision.

In monovision, one eye is corrected for near vision and the other remains suited for distance vision (typically the dominant eye). The patient adapts to both focal lengths and is able to have both near and distance vision. Monovision can be done through surgical means, such as presbyLASIK (corneal ablation), or corneal inlays, implanted in the cornea and aimed to produce a steeper central region or smaller aperture, increasing the depth of focus via the pinhole effect (Zare Mehrjerdi et al., 2017). Monovision is most often provided through intraocular lenses, and to a larger extent via contact lenses.

A more recent approach is multifocal intraocular lenses, which work using the simultaneous-image principle and consist of focusing objects of varying distances on different parts of the lens, allowing multiple focused images to be projected onto the retina. The drawback is that the focused image is superimposed with the blurred image from a different plane, causing some degradation to the overall image (Alio et al., 2017; Salerno et al., 2017).

\section{Dynamic corrections for presbyopia}

One of the proposals for dynamic correction of presbyopia is the removal of the aged, stiffer crystalline lens contents from the capsular bag, replacing it with a softer, deformable polymer or substance of sufficiently high refractive index. This technique is called lens refilling. A number of different substances have been used to replace lens contents, most typically a silicone polymer in viscous fluid form, which then gels inside 
the capsule. Some of these techniques have been tested and they find post-operatory accommodative range of 2-6 D (Koopmans et al., 2006; Nishi et al., 2008). However, this approach presents a number of problems. One is that preventing leakage of the material has not been entirely successful or practical. The other is capsular opacification that occurs after refilling. This opacification has an effect on static (by blocking light and lowering overall image quality) and dynamic (by resulting in increased stiffness of the overall lens and reduced accommodative amplitude) performance (Nibourg et al., 2015).

\section{Accommodating Intraocular Lenses}

Accommodating intraocular lenses are expected to be the ultimate correction for presbyopia, aiming at restoring the dynamic accommodation of young eyes. So far, few accommodating intraocular lenses have come out for mass-market use, and they are not truly 'accommodating,' in that the changes in power of the lens itself do not tend to be clinically relevant. In some approaches, movement of the optic(s) is meant to cause a change in overall power. The Crystalens (Bausch \& Lomb), an FDA-approved accommodating lens, offers a dynamic shift in focal length by shifting its single optical element forward and backward through specially designed haptic hinges (see Figure 1.7A). Although there were reported clinical outcomes that showed an improvement in near vision, experimental studies have shown that this mechanism did not function correctly for implanted lenses, and the movement was often in the opposite direction as the lens required (Koeppl et al., 2005; Marcos et al., 2014). It is likely that moderate improved vision at near may be associated to an increase in depth-of-focus, resulting from tilt and therefore coma in the lens (Pérez-Merino et al., 2014). Another similar single-optic accommodating lens was the Biocomfold, by Morcher. Experimental findings for these lenses also observed movement in the opposite direction as that predicted by the lens mechanism, and contradictory increases in pseudoaccommodation (Cleary et al., 2010; Legeais et al., 1999).

The Synchrony AIOL (VisionGen, AMO), a dual-optic AIOL that had a similar shifting mechanism, did not provide sufficient accommodation outcomes (Alió et al., 2012) and was eventually taken off the market. Another similar lens is the 1CU lens, a single optic that shifts axially. Similar to the Crystalens, some improved near vision outcomes were found, though it was also reported that excessive fibrosis led to posterior capsule 
opacification and the need for explantation (Mastropasqua et al., 2007). To the extent that improved visual outcomes have been found for these type of lenses, they are likely to be a result of pseudoaccommodation (static optical properties of the eye after lens implantation) rather than accommodation (a phenomenon reliant on the dynamic effects of the ciliary muscle movement on the lens) (Langenbucher et al., 2003).
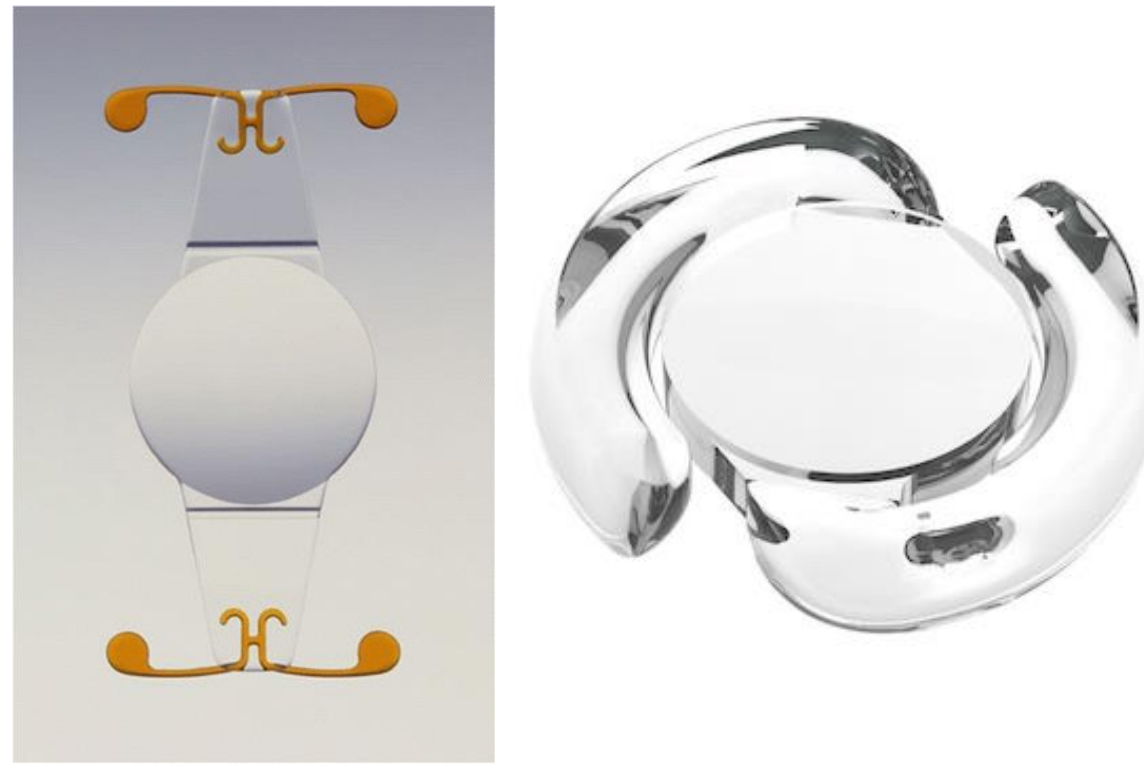

Figure 1.7: A) Crystalens. (Jorge L. Alió et al., 2017). B) FluidVision. (Alcon)

The Akkolens, or Lumina, is an accommodating intraocular lens consisting of two optical elements, the first a spherical lens and the second a cubic one. The lenses slide onto each other in order to create continuous focal shift. Results of clinical trials have recently been published, and show positive outcomes. The lens has not yet made it to market (Alió et al., 2018).

The FluidVision is another accommodating lens with dynamic range. It consists of a flexible shell-like optical element and two large hollow haptics. Both haptics and lens act as reservoirs for a fluid of high refractive index (see Figure 1.7B). Pressure on the haptics from the contraction of the ciliary muscle causes fluid to be displaced into the optic, resulting in an increase in curvature of the optic's surfaces and a corresponding change in power. Clinical trials for this lens remain underway; currently, the only published data relates to the long-term biocompatibility and stability of this lens (Kohl et al., 2014).

The NuLens is an accommodating that consists of an elastically deformable, hollow optical element with a silicone gel housed within it. Pistons, pushed by the ciliary 
muscle's contraction, apply pressure onto the silicone gel and allow the optic to curve further, which increases its power and results in increased accommodative amplitude. Surprisingly, despite the lens performing under a mechanism opposite to natural accommodation (more curved for relaxed accommodation and flatter to accommodate), clinical trials in 2009 showed promising results for visual acuity (Jorge Luciano Alió et al., 2009). However, the lens is still not on the market.

\subsection{Materials}

\subsubsection{Intraocular Lens and Contact Lens Materials}

The basic requirements for intraocular and contact lens materials are: biocompatible, stable, transparent, and mechanical properties suitable for wear.

The original material used for intraocular and contact lens application was polymethyl methacrylate (PMMA). This is an inert, transparent, rigid polymer. Its rigidity required a relatively large incision for intraocular implantation, and its limited oxygen permeability made it unsuitable for long-term contact lens use (Musgrave \& Fang, 2019).

Polymer hydrogels, originally based on hydroxyl ethyl methacrylate, were an improvement on PMMA. These materials are composed of hydrophilic monomers, allowing them to have higher water content and much less stiffness, allowing for foldable intraocular lenses and soft contact lenses. Additionally, their oxygen permeability is much improved compared to PMMA. For these polymer hydrogels, the mechanical and optical properties (Young's Modulus, tensile strength, elongation \% at break, refractive index) are a function of different factors: the co-polymers that compose the material; the amount of cross-linking induced in the polymerization process; and the final water content of the polymer (Musgrave \& Fang, 2019; Tranoudis \& Efron, 2004). This variability makes them suitable for both intraocular and contact lens applications, with polymers used for intraocular lenses typically having higher moduli (1-2 MPa), water content (20-30\%) and higher refractive indices (1.44-1.46) relative to polymers used for contact lenses (0.2-0.6 MPa, 38-70\% water content, 1.38-1.42 refractive index).

For contact lens applications, higher oxygen permeability was required, which led to the development of silicone-based co-polymers. The silicone monomer results in higher 
permeability, and a second, hydrophilic monomer counteracts the silicone's inherent hydrophobic character (which would otherwise result in tackiness and general discomfort during wear). These materials have lower water content and higher refractive index than hydrophilic materials.

\subsubsection{Silk}

Silk is a natural material, produced by a number of species (spider, bombyx mori silkworm), known for its mechanical toughness. Spider silk, in particular, is known for being tougher than materials such as Kevlar, is both strong and elastic, and considerably tougher than B. mori silk, but is largely inaccessible for large-scale applications due to the difficulties in obtaining the material from spiders (Römer \& Scheibel, 2008).

B. Mori silk has found applications as a biomaterial due to biocompatibility, accessibility (it is widely used in the textile industry and can be produced in bulk quantities), tunable properties, in vivo stability, and wide range of possible forms such as films, hydrogels, and sponges (as can be seen in Figure 1.8). B. mori silk is composed of fibroin protein coated by sericin protein. Sericin is believed to cause an autoimmune response when physically associated with fibroin (Panilaitis et al., 2003), is typically removed from the fibroin protein through the process of degumming, and the raw silk fibroin is used in different configurations. There has been interest and work in using silk as a potential scaffold for tissue engineering, as silk films have shown good attachment and growth promotion of for different types of cells and tissues, while allowing for control over the final mechanical and topographic properties of the scaffold (Altman et al., 2003).

The process of silk fibroin extraction and solution preparation involves degumming the fibroin from its sericin (using sodium carbonate and boiling water for $30 \mathrm{~min}$ to 3 hours depending on the desired molecular weight of the fibroin molecules), dissolving the fibroin with lithium bromide ( $\mathrm{LiBr}$ ), and dialyzing for two days until a silk solution of 6-10\% weight per volume remains, which is stable for up to a month at $4^{\circ} \mathrm{C}$ refrigeration (Rockwood et al., 2011).

Tissue engineering applications for ocular reconstruction have been considered, although the complexity of ocular structures makes this a more challenging problem. Silk poses a number of advantages when it comes to use for ocular tissue engineering. 
Its complex structure gives it multiple functional groups that can interact with other molecules, which could be tailored for specific needs. Silk films are already transparent, but they must preserve transparency after corneal stromal cells grow onto it. Their architecture and topography can be tuned, as well as their secondary structure, in order to encourage directional growth. They also have inherent mechanical strength that is higher than other scaffolding materials, which would preserve stability in the cornea (Harkin et al., 2011). Their main drawback is their low permeability, which must be offset through manufacturing techniques while maintaining other parameters such as transparency (Altman et al., 2003).

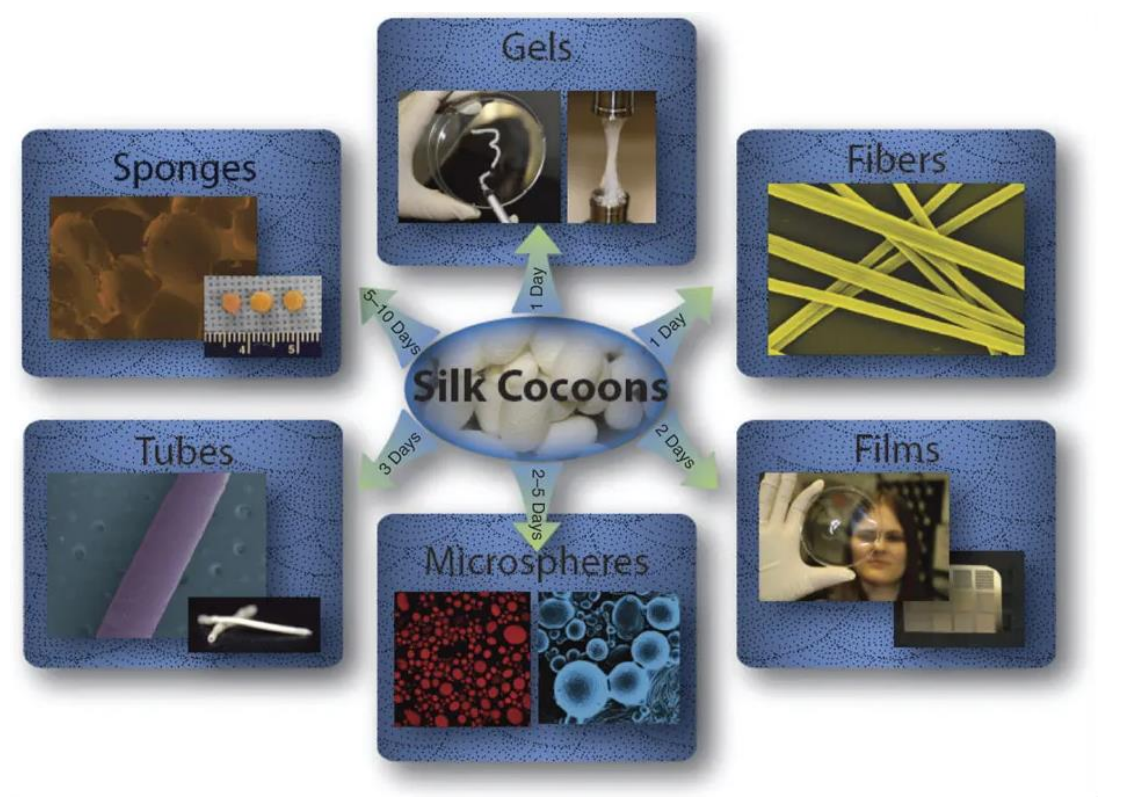

Figure 1.8: Different types of materials and structures derived from bombyx mori silk fibroin. (Rockwood et al., 2011)

\subsection{Optical Imaging Technologies}

\subsubsection{Optical Coherence Tomography}

Optical coherence tomography is an imaging technique that creates cross-sectional images of three-dimensional objects. The technique is based on low coherence interferometry, typically in a Michelson interferometer configuration. A light source is split by a beam splitter, with half of the beam reflecting off a reference mirror, and the other half reaching a sample surface. Light scattered from this sample surface is reflected back to the beam splitter. When recombined, the light from both arms 
produces an interference signal that is recorded by a detector unit. There are different types of OCT techniques used to obtain a depth scan.

Time Domain OCT generates a depth profile by axially translating the reference mirror to generate interferometric fringes for different depths. It typically uses a Michelson interferometer configuration and a broadband light source. In order to obtain a length profile the sample arm will have a transverse scanning system that directs the beam across the length of the sample (and across, for a fully three dimensional profile).

Spectral domain OCT generates a depth profile not by translation of the reference mirror, but by recording the intensity as a function of the wavelengths of the light source. A spectrometer is required to decode the spectral interferogram, and the backscattered depth profile is obtained after performing a Fourier transform on it.

A more in-depth overview of OCT technology and technique can be found in Optical Coherence Tomography: Development, Principles, and Applications (Fercher, 2010).

OCT has found widespread usage in ophthalmology. Its initial uses were limited to the posterior segment of the eye, to image the macula, retina, and ocular nerve. A thorough review of posterior segment applications can be found in Optical coherence tomography - current and future applications (Adhi \& Duker, 2013). OCT has also been extensively used for anterior segment imaging, in clinical and research contexts. A thorough review of anterior segment applications can be found in Anterior segment optical coherence tomography and its clinical applications (S B Wang et al., 2019).

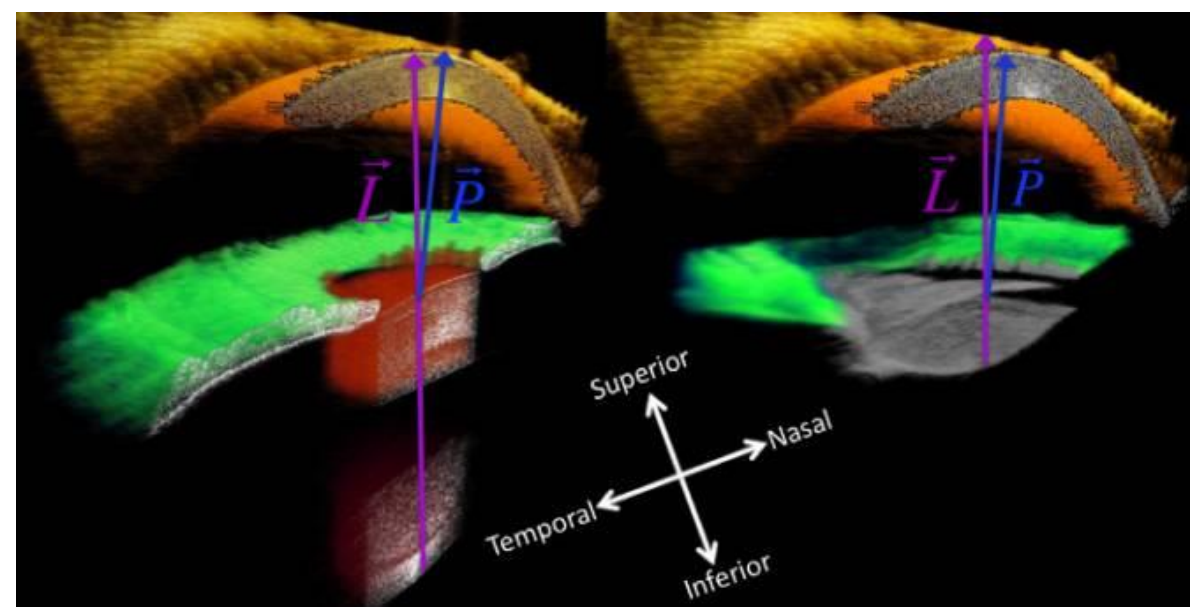

Figure 1.9: Anterior segment reconstruction with OCT (Ortiz et al., 2013). 
Raw images obtained from OCT are not fully quantitative, as they suffer from fan distortion (arising from the scanner architecture) and optical distortions (as surfaces such as the posterior cornea or the lens surfaces are imaged through refractive surfaces). Quantification algorithms have been developed that correct for these distortions as well as allow automatic signal processing from the images, extracting 3D elevation maps of ever surface of the cornea, iris, and lens (Ortiz et al., 2009). 3D quantitative OCT has been used in a research context to investigate various aspects of the behavior and anatomy of anterior segment tissues. Applications include: topographic analysis of patient corneas (Ortiz et al., 2011), keratoconic corneas implanted with intracorneal ring segments (Pérez-Merino et al., 2013), and corneas under air puff pressure (Dorronsoro et al., 2012); quantification of crystalline lens shape changes during accommodation (Gambra et al., 2013; Martinez-Enriquez et al., 2017), and reconstruction of crystalline lens' refractive index gradient (de Castro et al., 2019). Figure 1.9 shows an OCT reconstruction of all anterior segment tissues.

\subsubsection{Brillouin Optical Microscopy}

Brillouin light scattering is the inelastic scattering of electromagnetic waves after interacting with the acoustic modes of materials. The exchange of energy between photons and the acoustic waves results in a frequency shift between incident and scattered light, which is related to the mechanical properties of a material in the following way:

$$
\omega_{b}=\frac{2 n}{\lambda} \sqrt{\frac{M}{\rho}} \sin \frac{\theta}{2}
$$

Where $\omega_{b}$ is the frequency shift, $\mathrm{n}$ and $\omega_{b}$ are the refractive index and density of the sample, $\lambda$ and $\theta$ are the wavelength of the light source and the angle between incident and scattered wave vectors, and $M$ is the longitudinal modulus.

Brillouin optical microscopy is a non-invasive, non-destructive technique in which an imaging device based on Brillouin light scattering detects local mechanical properties of a tissue or material by measuring the frequency shift. This type of device is of particular interest for studies of the anterior segment of the eye, as the cornea and crystalline lens have been shown to have spatial and subject-specific variations in mechanical properties, believed to relate to various pathologies such as presbyopia and keratoconus. 
A number of studies have been done on crystalline and corneal structures using Brillouin optical microscopy techniques. Reiß and co-authors measured the longitudinal modulus for ex-vivo for rabbit and porcine eyes (Reiß et al., 2011). Bailey and coauthors did the same for ex-vivo human lenses ranging between 30 and 70 years of age (Bailey et al., 2010). Scarcelli and co-authors applied the technique in vivo for murine and porcine lenses and found an increase in longitudinal modulus with age, as well as a correlation between longitudinal modulus and experimentally measured Young's Modulus for lens samples (Scarcelli et al., 2011). Further work by Besner and Scarcelli for in vivo human lenses did not find an age-related increase in the longitudinal modulus of crystalline lenses (Besner et al., 2016).

In the cornea, a number of studies have looked at changes in stiffness in keratoconic corneas (Scarcelli et al., 2015; Shao et al., 2019), after corneal crosslinking (Scarcelli et al., 2013), and the effect of corneal hydration on Brillouin microscopy measurements (Shao et al., 2018). Figure 1.10 shows a representation of measured Brillouin frequency shift in a cornea.

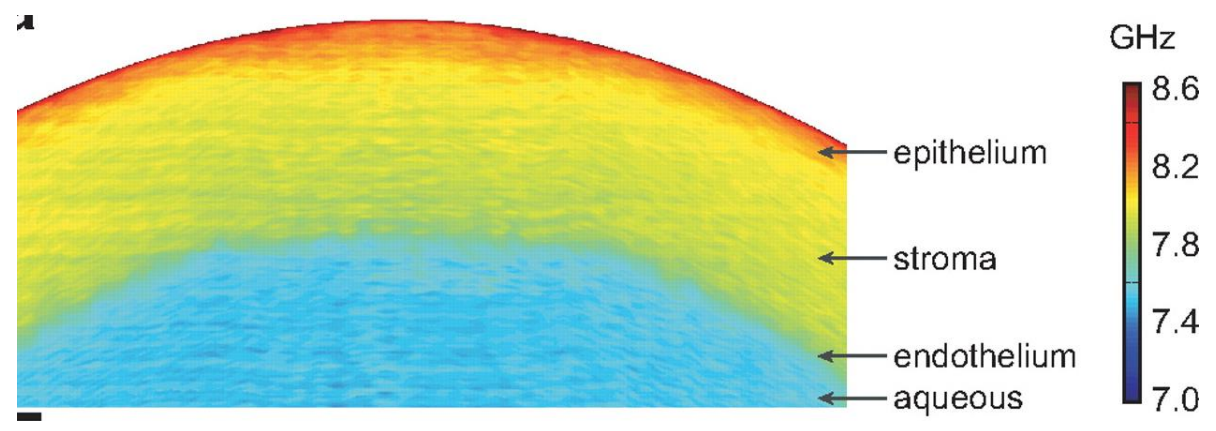

Figure 1.10: Brillouin frequency shift in cornea (Scarcelli et al., 2012).

The difficulty in Brillouin optical microscopy for material characterization lies in the interpretation of the data and what underlying mechanics are being measured. Biological tissues have high levels of water content; as the Brillouin shift measures the compressibility of the tissue, the measurements obtained can be highly dependent on the differences in hydration throughout the tissue rather than inherent stiffness (Wu et al., 2018). However, hydration is not entirely separate from mechanical properties, and there is some evidence that Brillouin optical microscopy is able to detect anisotropy in hydrated materials (Koski et al., 2013), meaning the frequency shift is not entirely dependent on the bulk properties of the fluid. Through theoretical material models, the 
solid and liquid mechanical properties can potentially be separated (Scarcelli \& Yun, 2018).

\subsection{Continuum Mechanics}

Continuum mechanics is the study of the mechanical behavior of materials when treated as a continuous solid of matter, i.e. ignoring its composition of particles / molecules. Thus, infinitesimal elements of the whole carry its bulk properties. A more detailed discussion and derivation of these concepts can be found in Introduction To Continuum Biomechanics (Athanasiou \& Natoli, 2008) and Biosolid Mechanics Lecture Notes (Hollister).

\subsubsection{Stress, strain, and displacement}

Two types of forces act on solid bodies. The first, body forces, are those acting uniformly on the entire volume. Gravity and electromagnetic are examples of body forces. The second, surface forces, are those that are localized or distributed on a region of the solid's surface. Contact between two objects is an example of a surface force.

When a body is constrained, and a force is applied to it, stresses (or internal force intensity) develop within the body as a result of these external forces. Those forces must be equivalent for the body to remain in equilibrium. For a three-dimensional object, these internal forces can be represented by a traction vector force acting on the body; the three vector forces acting on the surfaces can be decomposed to their components in an $x-y-z$ coordinate system. The nine components can be expressed as a second order tensor known as the Cauchy stress tensor, $\sigma_{i j}$. The stress tensor is symmetric, so $\sigma_{i j}=$ $\sigma_{j i}$. The equilibrium equations for stress are:

$$
\frac{\partial \sigma_{i j}}{\partial x_{i}}+b_{j}=0
$$

Where $x_{i}$ describes position in the deformed configuration and $b_{j}$ is a body force.

The stress equilibrium equations, on their own, are indeterminate, and cannot be used to directly solve for material stresses. Information on how the material deforms is required to solve the equations.

There is a tensor for the condition where deformation is small $(<1 \%)$, the small deformation strain tensor, a linear tensor defined as: 


$$
\varepsilon_{i j}=\frac{1}{2}\left(\frac{\partial u_{i}}{\partial x_{j}}+\frac{\partial u_{j}}{\partial x_{i}}\right)
$$

Where $u_{i}$ is the displacement and $x_{i}$ describes position in the deformed configuration.

In order to describe large deformation, other tensors are required. First, a tensor that describes the relationship between reference and deformed configurations. This is the deformation gradient tensor $F$, a non-symmetric tensor defined as:

$$
F_{i j}=\frac{\partial x_{i}}{\partial x^{\prime}}
$$

where $x^{\prime}$ is the reference configuration and $x$ is the deformed configuration of the body, which can also be expressed in terms of displacement and reference configuration:

$$
F_{i j}=\delta_{i j}+\frac{\partial u_{i}}{\partial x_{j}^{\prime}}
$$

The deformation gradient can also be presented in terms of rotation (via rotational tensor $R$ ) and stretch (via stretch tensor $V$ or $U$ ):

$$
\begin{aligned}
& F_{i j}=R_{i k} U_{k j} \\
& F_{i j}=V_{i k} R_{k j}
\end{aligned}
$$

Using the deformation gradient tensor, a finite strain tensor $E$ (for strains $>1 \%$ ) can be defined:

$$
E_{i j}=\frac{1}{2}\left(F_{k i} F_{k j}-\delta_{i j}\right)
$$

This finite strain tensor can also be described in terms of displacements and reference configurations:

$$
E_{i j}=\frac{1}{2}\left(\frac{\partial u_{i}}{\partial x_{j}^{\prime}}+\frac{\partial u_{j}}{\partial x_{i}^{\prime}}+\frac{\partial u_{k}}{\partial x_{i}^{\prime}} \frac{\partial u_{k}}{\partial x_{j}^{\prime}}\right)
$$

The finite strain tensor has a quadratic element, which makes it non-linear. It is defined using the reference configuration $x^{\prime}$, unlike the small strain tensor, which is defined using the deformed configuration $x$.

Two common tensors are the right and left Cauchy deformation tensors, described in terms of the deformation gradient tensor, as: 


$$
C_{i j}=F_{k i} F_{k j}
$$

(Right Cauchy deformation tensor)

$$
B_{i j}=F_{i k} F_{j k}
$$

(Left Cauchy deformation tensor)

The Cauchy stress tensor and small strain tensor are both defined in the deformed coordinate system; as such, they can be used together. The finite strain tensor is defined for the reference coordinate system, and thus requires different stress tensors. Those are the Piola-Kirchoff stress tensors.

The $1^{\text {st }}$ Piola-Kirchoff stress tensor is defined as:

$$
T_{i j}=\sigma_{i k} J\left(F_{j k}\right)^{-1}
$$

The $2^{\text {nd }}$ Piola-Kirchoff stress tensor is defined as:

$$
S_{i j}=\left(F_{i r}\right)^{-1} \sigma_{r k} J\left(F_{j k}\right)^{-1}
$$

Where $J=\operatorname{det} F$.

The corresponding equilibrium equations for the PK tensors are:

$$
\begin{gathered}
\frac{\partial T_{i j}}{\partial x_{j}^{\prime}}+p^{\prime} b^{\prime}{ }_{i}-p^{\prime} \frac{d^{2} x_{i}^{\prime}}{d t^{2}}=0 \\
\frac{\partial\left(F_{k i} S_{k j}\right)}{\partial x_{j}^{\prime}}+p^{\prime} b^{\prime}{ }_{i}-p^{\prime} \frac{d^{2} x_{i}^{\prime}}{d t^{2}}=0
\end{gathered}
$$

\subsubsection{Constitutive models}

Strain displacement and equilibrium equations are still indeterminate at this point and require additional equations in order to solve. These are constitutive equations, which describe the relationship between stress and strain, with variables that are dependent on the properties of the material.

An elastic material deforms after loading and returns to its initial shape when the load is removed. These materials store, but do not dissipate energy. A strain energy function $(W)$ can be defined for this material, and a relationship between stress and strain is defined as: 


$$
\begin{gathered}
\sigma_{i j}=\frac{\partial W\left(a_{i}, \varepsilon_{i j}\right)}{\partial \varepsilon_{i j}} \\
\text { infinitesimal strain } \\
S_{i j}=\frac{\partial W\left(a_{i}, E_{i j}\right)}{\partial E_{i j}}
\end{gathered}
$$

\section{finite strain tensor}

A constitutive equation for linear elasticity is derived from Hooke's Law and is in the form:

$$
\sigma_{i j}=C_{i j k l} \varepsilon_{k l}
$$

$C_{i j k l}$ has 81 constants for a three-dimensional case and 16 for a two-dimensional case. However, a number of symmetries (strain symmetry, stress symmetry, and strain energy symmetry) can reduce the number of constants to 6 independent constants for $2 \mathrm{D}$ and 21 for 3D (which can be further reduced to 2, 5, and 9 due to material symmetry).

For non-linear elastic materials, the relationship between strain energy function and stress (for $1^{\text {st }} \mathrm{PK}$ stress tensor) is:

$$
S_{i j}=\frac{\partial W}{\partial E_{i j}}
$$

Which can be also be expressed in terms of the right Cauchy deformation tensor:

$$
S_{i j}=2 \frac{\partial W}{\partial C_{i j}}
$$

This definition is often called the Doyle-Ericksen formula (Doyle \& Ericksen, 1956).

Invariants are functions of tensors that do not vary when evaluated under different coordinate systems. The definition of the invariants for a second-order tensor $A$ is:

$$
\begin{gathered}
I_{1}(A)=A_{i i} \\
I_{2}(A)=\frac{1}{2}\left(A_{i i} A_{j j}-A_{j i} A_{i j}\right) \\
I_{3}(A)=\operatorname{det}(\boldsymbol{A})
\end{gathered}
$$


For non-linear elastic materials, a strain energy function can be defined in terms of its three invariants:

$$
W=W\left[I_{1}\left(C_{i j}\right), I_{2}\left(C_{i j}\right), I_{3}\left(C_{i j}\right)\right]
$$

Non-linear elastic models are also often defined in terms of the principal stretches, which leads to the following definitions for first and second PK tensors and Cauchy stress tensor:

$$
\begin{gathered}
S_{i}=\frac{1}{\lambda_{i}} \frac{\partial W}{\partial \lambda_{i}} \\
T_{i}=\frac{\partial W}{\partial \lambda_{i}} \\
\sigma_{i}=J^{-1} \lambda_{i} \frac{\partial W}{\partial \lambda_{i}}
\end{gathered}
$$

For soft tissues and polymers, it is often the case that the material is incompressible, meaning its volume does not change regardless of forces applied or resulting deformation. For this kind of material, the strain energy function requires an additional term, an incompressibility condition:

$$
W=W(F)+p(J-1)
$$

$p$ is a Lagrange multiplier and represents the hydrostatic pressure. $J$, the determinant of $F$, has a physical meaning: it is the ratio of volume in deformed and reference configurations. For an incompressible material, $J$ must equal 1 . The corresponding equations for stress (PK, Cauchy) for an incompressible material are:

$$
\begin{gathered}
S_{i}=-\frac{1}{\lambda_{i}^{2}} p+\frac{1}{\lambda_{i}} \frac{\partial W}{\partial \lambda_{i}} \\
T_{i}=-\frac{1}{\lambda_{i}} p+\frac{\partial W}{\partial \lambda_{i}} \\
\sigma_{i}=-p+\lambda_{i} \frac{\partial W}{\partial \lambda_{i}}
\end{gathered}
$$

For the left Cauchy tensor, the invariants are:

$$
I_{1}=\lambda_{1}^{2}+\lambda_{2}^{2}+\lambda_{3}^{2}
$$




$$
\begin{gathered}
I_{2}=\lambda_{1}^{2} \lambda_{2}^{2}+\lambda_{2}^{2} \lambda_{3}^{2}+\lambda_{3}^{2} \lambda_{1}^{2} \\
I_{3}=\lambda_{1}^{2} \lambda_{2}^{2} \lambda_{3}^{2}
\end{gathered}
$$

Different types of constitutive models have been developed over the years. They can be broadly categorized as mechanistic models, which seek to describe the data through a representation of its underlying mechanical phenomena, and phenomenological models, which simply seek to best fit the experimental data. Phenomenological models include the Mooney-Rivlin, Ogden, and Yeoh models. Mechanistic models include the Arruda Boyce model (Ali et al., 2010).

The Mooney-Rivlin constitutive model is defined by the strain energy function:

$$
W=C_{1}\left(I_{1}-3\right)+C_{2}\left(I_{2}-3\right)+D_{1}(J-1)^{2}
$$

Where $C_{1}$ and $C_{2}$ are material constants, which must be obtained from experimental evaluation of strain response to stress, and D relates to the volumetric response. For an incompressible material, $\mathrm{D}_{1}=0$.

The Neo-Hookean constitutive model is a special case of the Mooney-Rivlin model, where $\mathrm{C}_{2}=0$.

Simplification of experimental conditions and symmetries allow us to define constitutive equations and material parameters in simpler forms.

Under small strain, the stress-strain curve is often linear. In this case, linear constitutive models can be used.

The Young's Modulus $(E)$ is defined as the relationship between the stress and strain from a force applied normal to a plane, using Hooke's Law.

$$
\sigma=E \varepsilon
$$

Poisson's Ratio, $v$, is the ratio of the contraction of a material in its transverse direction when undergoing a normal strain.

$$
v=-\frac{\varepsilon_{x}}{\varepsilon_{y}}=-\frac{\varepsilon_{x}}{\varepsilon_{z}}
$$

Poisson's Ratio can range from 0 to 0.5 . 
Shear stress $(\tau)$ and strain $(\gamma)$ are the response to a force applied parallel to a plane:

$$
\tau=G \gamma
$$

The shear modulus $(G)$ can be related to the Young's Modulus with the following equation:

$$
G=\frac{E}{2(1+v)}
$$

The bulk modulus, $K$, defines the effect of an external pressure on the volume of a substance. It can be defined in terms of $E$ and $v$ :

$$
K=\frac{E}{3(1-2 v)}
$$

It can be seen from this relationship that when a material is incompressible ( $v$ approaches 0.5), its bulk modulus is much greater than its Young's Modulus. Although 0.5 would represent an incompressible material, it is not mathematically appropriate to use this, as this expression approaches infinity; instead, hyperelastic models with incompressible terms should be used.

The p-wave or Longitudinal Modulus $(M)$ is defined as the ratio of axial stress to axial strain in a situation where all other strains are zero. It is related to the Bulk modulus by the following equation:

$$
M=\frac{3 K(1-v)}{1+v}
$$

It can be seen from this relationship that when a material is incompressible, $K$ and $M$ will be equal.

\subsubsection{Fitting material data to constitutive models}

For uniaxial stress, the principal stretch ratios can be simplified to:

$$
\lambda_{1}=\lambda, \lambda_{2}=\lambda_{3}=\frac{1}{\sqrt{\lambda}}
$$

Which means the invariants can be expressed in the following way:

$$
I_{1}=\lambda^{2}+\frac{2}{\lambda^{\prime}} I_{2}=2 \lambda+\frac{1}{\lambda^{2}}, I_{3}=1
$$

For uniaxial stress, the Cauchy stress tensor reduces to: 


$$
\sigma_{11}=2\left(\lambda+\frac{1}{\lambda^{2}}\right)\left(\frac{\partial W}{\partial I_{1}}+\frac{1}{\lambda} \frac{\partial W}{\partial I_{2}}\right)
$$

With this definition, an equation for fitting stress and strain (stretch ratio) data for different material models can be obtained. Uniaxial tensile tests are commonly used in materials characterization due to their configuration allowing for this reduction of independent parameters in constitutive models.

\section{Neo-Hookean Model}

The energy function for a 1-term Neo-Hookean model is defined as:

$$
W=\frac{\mu}{2}\left(I_{1}-3\right)
$$

And its partial derivative is:

$$
\frac{\partial W}{\partial I_{1}}=\frac{\mu}{2}
$$

After substituting 1.46 in 1.44 , the relationship between engineering stress and stretch ratio for a Neo-Hookean model is obtained:

$$
\sigma=\left(\lambda-\frac{1}{\lambda^{2}}\right)(\mu)
$$

For small deformations, this equation simplifies to $\sigma=3 \mu \varepsilon$. This corresponds to Equation 1.39, where $v=0.5$ for incompressibility, relating the Neo-Hookean coefficient and the shear modulus.

\section{Mooney-Rivlin Model}

\section{2-Parameter Model}

The energy function for a 2-term Mooney Rivlin model is:

$$
W=C_{10}\left(I_{1}-3\right)+C_{01}\left(I_{2}-3\right)
$$

And its partial derivatives are:

$$
\frac{\partial W}{\partial I_{1}}=C_{10} \quad \frac{\partial W}{\partial I_{2}}=C_{01}
$$

After substituting, the relationship between engineering stress and stretch ratio for a 2 term Mooney Rivlin model is found to be: 


$$
\sigma=2\left(\lambda-\frac{1}{\lambda^{2}}\right)\left(C_{10}+\frac{C_{01}}{\lambda}\right)
$$

\section{3-parameter model}

The energy function for a 3-term Mooney Rivlin model is defined as:

$$
W=C_{10}\left(I_{1}-3\right)+C_{01}\left(I_{2}-3\right)+C_{11}\left(I_{1}-3\right)\left(I_{2}-3\right)
$$

And its partial derivatives are:

$$
\frac{\partial W}{\partial I_{1}}=C_{10}+C_{11}\left(I_{2}-3\right) \frac{\partial W}{\partial I_{2}}=C_{01}+C_{11}\left(I_{1}-3\right)
$$

Substituting the partial derivative of the invariants into 1.44 , the relationship between engineering stress and stretch ratio for a 3-term Mooney Rivlin model is found:

$$
\sigma_{e}=2\left(\lambda-\frac{1}{\lambda^{2}}\right)\left(C_{10}+\frac{C_{01}}{\lambda}+3 C_{11}\left(\lambda+\frac{1}{\lambda^{2}}+\frac{1}{\lambda}-1\right)\right)
$$

\section{Yeoh Model}

\section{2-parameter model}

The energy function for a 2-term Yeoh model is defined as:

$$
W=C_{10}\left(I_{1}-3\right)+C_{20}\left(I_{1}-3\right)^{2}
$$

And its partial derivatives are:

$$
\frac{\partial W}{\partial I_{1}}=C_{10}+C_{20}\left(I_{1}-3\right)
$$

After substituting the partial derivative of the invariant into 1.44, the relationship between engineering stress and stretch ratio for this model is found:

$$
\sigma=2\left(\lambda-\frac{1}{\lambda^{2}}\right)\left(C_{10}+2 C_{20}\left(\lambda^{2}+\frac{2}{\lambda}-3\right)\right)
$$

\section{3-parameter model}

The energy function for a 3-term Yeoh model is defined as:

$$
W=C_{10}\left(I_{1}-3\right)+C_{20}\left(I_{1}-3\right)^{2}+3 C_{30}\left(I_{1}-3\right)^{3}
$$


And its partial derivative is:

$$
\frac{\partial W}{\partial I_{1}}=C_{10}+C_{20}\left(I_{1}-3\right)+C_{30}\left(I_{1}-3\right)^{2}
$$

After substituting the partial derivative of the invariant into 1.44 , the relationship between engineering stress and stretch ratio for a 3-term Yeoh model is found to be:

$$
\sigma=2\left(\lambda-\frac{1}{\lambda^{2}}\right)\left(C_{10}+2 C_{20}\left(\lambda^{2}+\frac{2}{\lambda}-3\right)+3 C_{30}\left(\lambda^{2}+\frac{2}{\lambda}-3\right)^{2}\right)
$$

\subsection{The Finite Element Method}

\subsubsection{Principles}

The equilibrium equations for continuum mechanics are partial differential equations. Various methods have been developed to solve for systems of partial differential equations, one of the most prominent ones being the Finite Element Method, or FEM. FEM was developed to solve complex structural mechanics problems (Turner et al., 1956).

The method requires discretizing a geometry into 'finite elements,' which represent an approximation of the displacement field. A 'test' function that represents this approximation is substituted into the partial differential equations, in order to convert them to algebraic equations, which can be solved using conventional numerical methods.

For the finite element approximation, the equilibrium problem is multiplied by a virtual displacement (the test function) and integrated over the length of the continuum. The constitutive equation and strain displacement relationship can be substituted into this integral, and boundary conditions and an approximation of the test function can be used to convert the problem into a set of linear equations. The system of linear equations for a linear constitutive material (Young's Modulus) and a 1D problem is:

$$
\begin{gathered}
\frac{d}{d x} \sigma=0=\int_{0}^{L} \frac{d v}{d x}\left(E A \frac{d u}{d x}\right) d x+v E A \frac{d u}{d x}(L) \mid=0 \\
\sum_{n=1}^{\# \text { elements }}\left(\frac{E A}{L}\left[\begin{array}{cc}
1 & -1 \\
-1 & 1
\end{array}\right]\left\{\frac{u_{1}}{u_{2}}\right\}-\left\{\frac{f_{1}}{f_{2}}\right\}\right)=0
\end{gathered}
$$


Where $E$ (Young's Modulus), $A$ (Area), $L$ (Length), and $f$ (force) are input conditions for the problem and must be given in order to solve. The $E A / L$ term is defined as the stiffness matrix; thus, the global set of linear equations can be described as:

$$
[K]\{u\}=\{f\}
$$

This set of linear equations is solved for all finite element approximations (even in nonlinear problems, as those are also approximated to a linear set).

The finite element method can be described by the following steps:

- The geometry is discretized into elements with set number of nodes.

- Interpolation functions are used to interpolate the field variable over the element, typically polynomial functions.

- The matrix equation, which relates nodal values of the unknown function to other parameters, is established. The most used approaches are the Galerkin method (if the physical formulation of the problem is known as a differential equation) and the variational formulation (if the physical problem can be formulated as minimization of a functional).

- The global equation system is assembled from the local element equations, based on element connectivities, and boundary conditions are imposed on nodes or geometries.

- The global equation system is solved, using direct or iterative methods.

A detailed explanation of the mathematics underpinning the method can be found in The Finite Element Method (Zienkiewicz et al., 2005).

Finite element software can be programmed by researchers and engineers. More common is the use of commercial finite element solvers with pre-programmed formulation methods, constitutive models, and element formulations. The finite element method has been used extensively in biomedical research (sources), including ophthalmology. The cornea and crystalline lens are both mechanical structures under loading, and FEM has been used to predict their response to loading (Burd et al., 2002; Crouch et al., 2004; Hermans et al., 2006; Kling et al., 2014; Lanchares et al., 2012; Z Liu et al., 2006; Ljubimova et al., 2008; Nejad et al., 2014; T D Nguyen \& Boyce, 2011; K Wang et al., 2016). 


\subsubsection{Inverse Modeling}

The standard approach in commercial finite solvers is to describe the problem in terms of initial geometry, boundary conditions, and constitutive models, and solve. The solution yields the displacement matrix and other relevant parameters can be calculated (stress, strain, forces). This approach is effective for engineering problems and product design, as it predicts behavior under loading. The input to the model is known or easily measurable compared to the output.

In biomedical engineering, this is not always the case. Although tissues can be characterized using standard mechanical testing, this is not suitable for diagnostics, as these are destructive approaches. It is possible to measure tissue deformation under nondestructive, non-invasive loading, but such loading is typically complex, making it impossible to reduce the number of independent variables (1.18) and analytically describe the material.

Inverse modeling is an iterative approach that uses the finite element method to resolve for unknown conditions or parameters in the problem formulation (Dennis et al., 2011). Measurements of the displacement of a tissue or structure under loading are obtained and quantified. A finite element model of this same problem is built. The model does not have sufficient input to be solved; a variable value is assigned to the unknown input property. In biomedical research, this unknown variable is the constitutive model that describes the stress-strain response, the boundary conditions of the solution, or both. The model is solved, and its displacement is compared to the experimentally measured displacement. An algorithm is used to iteratively solve the model, using the comparison between simulated and experimental displacement as a merit function to minimize, and the constitutive model coefficients as variables.

This approach has been used in ophthalmology research to assess mechanical properties of corneas (Kling et al., 2014; T D Nguyen \& Boyce, 2011; Sinha Roy et al., 2013) and crystalline lens (Reilly \& Cleaver, 2017).

\subsection{Hypothesis}

- Inverse modeling can be used to accurately retrieve mechanical properties of crystalline lens and cornea from experimental measurements. 
- These retrieved mechanical properties accurately describe the general behavior of the cornea and crystalline lens.

- Rose Bengal photobonding can be used as a tool for treatment of ocular conditions such as corneal wound healing and presbyopia.

- An accommodating lens that mimics the behavior of the crystalline lens can restore focusing range in presbyopic eyes.

\subsection{Objectives}

- To design an inverse modeling process that retrieves mechanical properties from cornea and crystalline lenses, and compare the results to properties retrieved from other experimental methods.

- To design an accommodating lens that changes the shape of its surfaces in response to a force, resulting in a change in power.

- To develop an experimental testing setup for evaluating power changes in an accommodating lens prototype.

- To evaluate the viability of photochemically bonding silk onto the cornea for applications such as corneal wound healing.

The thesis will advance the state of the art by 1) developing and validating inverse modeling methods, 2) developing and validating accommodating lens evaluation and design methods, 3 ) showing the viability of a photochemical technique to combine with the use of silk as an ocular bio-material. 


\section{Methods}

This chapter presents an overview of the devices and processes used to develop the work of the thesis.

\subsection{Hardware}

\subsubsection{Accommodation System}

The accommodation system is a combination of different optical and mechanical technologies. It was built to analyze the behavior of accommodating lens prototypes and compare their results to predicted behavior.

\section{Radial stretcher}

The primary component of the system is a radial stretcher mechanical device. This type of device is conceived for simulating accommodation of the eye and multiple designs have been developed over the years by a number of research groups. The version used for the work in this thesis was custom-developed specifically for this application. A precursor of this system is the EVAS II system, developed at the Bascom Palmer Eye Institute of the Miami University (Ehrmann et al., 2004).

The device consists of eight arms arranged radially around a central area that can hold a sample, which exert a pull on the sample and record the corresponding force. Eight arms is the conventional number used for this type of device (R. F. Fisher, 1977; Glasser \& Campbell, 1998), as well as the number of fixation points that the samples to be measured were expected to have. Although 8 arms are only an approximation for a fully equibiaxial load, calculations suggest the differences can be as little as $2 \%$ (Ehrmann et al., 2004). 16 arms have been used before, for full characterization of the elastic constants of elastomeric materials, where the relationship between force and stresses at all directions must be fully accounted for (Day \& Miller, 2000).

The base of each arm is a translation stage (M-111 Compact Micro-Translation Stage, PhysikInstrumente). The translation stage has a total displacement range of $15 \mathrm{~mm}$ and a minimum step size of $50 \mathrm{~nm}$. An aluminum plate is screwed onto the stage, and a force transducer (Fort10g, WPI) is mounted on top of it. The force transducer uses a semiconductor strain gauge; when its sensing leaf is displaced due to forces acting on it, 
a linear output force voltage is produced. The sensors used have a range of $10 \mathrm{~g}$ and a resolution of $<1 \mathrm{mg}$. Leaf springs are screwed on at the front and back of the inferior aluminum plate; a second, thin aluminum structure is also screwed on at the top. A wire is screwed onto the sensing leaf of the force transducer and the superior aluminum plate. An L-shaped base is screwed in front of the superior aluminum plate. A fixation shoe is attached to the L-shaped base's lower part. The top part of this fixation shoe is removable and fastened by a screw, allowing the sample to be clamped between both pieces.

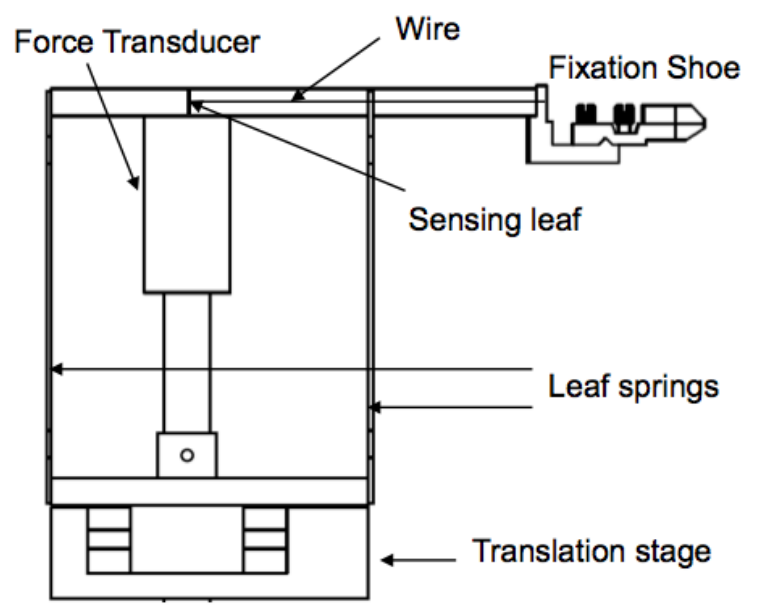

Figure 2.1: Schematic of stretcher arm. The translation stage displaces the structure and allows for a foce to be applied onto a sample attached to the fixation shoes. The force transducer is able to record this force. The system has eight separate stretcher arms aligned radially.

Both the translation stages and their corresponding force transducers are connected to a computer and their output can be registered. A computer program was designed in order to control the system. The user inputs a displacement (in $\mathrm{mm}$ ) for the stretcher's arms; the translation stage receives this input and applies the corresponding movement. The force transducer's sensing leaf generates a voltage from the applied force; this is the system's output.

The displacement of the translation stage is not equivalent to the displacement applied to the sample, due to the deflection of the leaf springs. Although strategies could be devised to monitor the true displacement applied to the sample, for the purposes of this work the system only has one output: the force from each individual transducer. Customized software was developed to control the displacement of the arms and obtain the force values corresponding to the voltage from the sensor. 


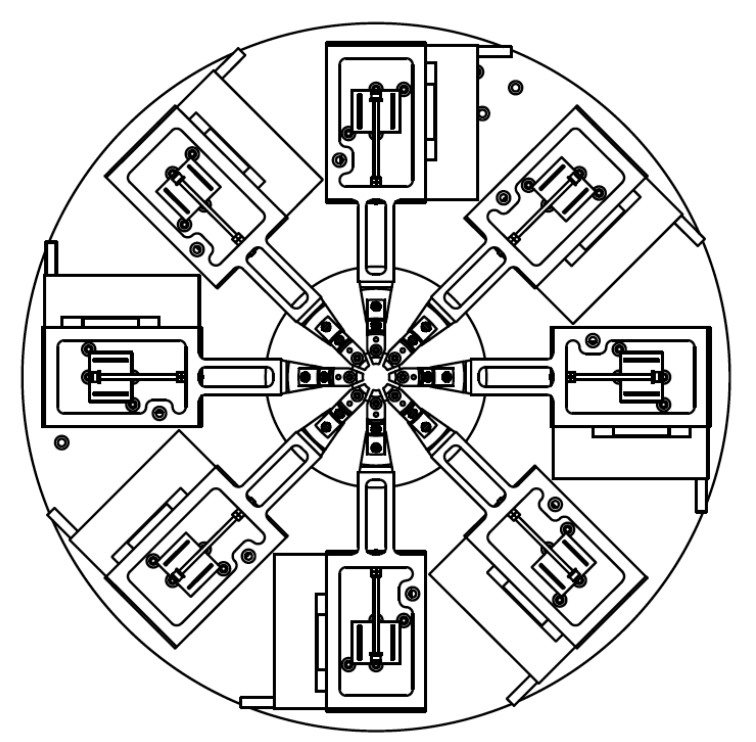

Figure 2.2. Schematic view of stretcher device from above.

In order to convert the voltage output to a force value, a calibration was done on the system. For this calibration, glass fibers of a known Young's Modulus (70 GPa) were stretched for each arm pair; the strain was measured using high-resolution photographs of each loading step. The force for each strain value was calculated from the known Young's Modulus and cross-sectional area of the fiber, and compared to the voltage reading from the system. The average value obtained was $0.0013 \mathrm{~N} / \mathrm{mV}$. The same procedure was repeated for a hydrophilic polymer of known strain-stress relationship. The average difference between the calibration values calculated for both measurements was under $5 \%$.

Below the fixation shoes of the stretcher, a plastic container of $100 \mathrm{~mm}$ diameter and 25 $\mathrm{mm}$ height was mounted. The central $3 \times 5 \mathrm{~mm}$ region of this container is a thin glass panel and allows for light to go through. This container can be filled with fluid during the experiments in order to have the samples properly hydrated.

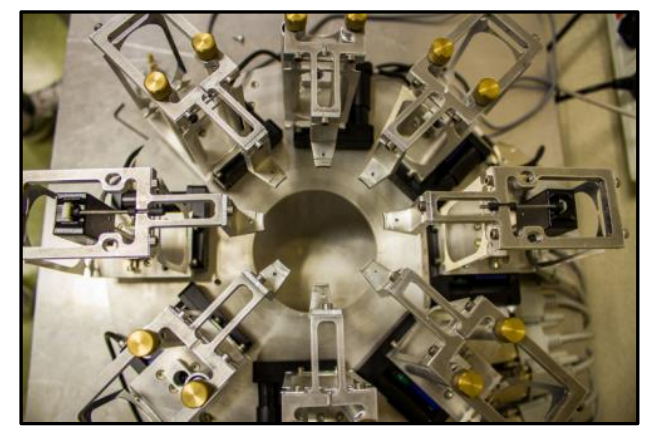

Figure 2.3: Radial stretcher. 


\section{Laser Ray Tracer System}

A Laser Ray Tracer system was incorporated onto the stretcher setup in order to measure the changes in lens power upon deformation. This system is an adaptation of a system used previously in the VioBio lab (Birkenfeld et al., 2013). The light source for the system is a HeNe laser $(\lambda=632 \mathrm{~nm})$ with a beam diameter of $1 \mathrm{~mm}$. A 2-D galvanometric scanning system (Mod. 6210, Cambridge Technology, Medford MA) and a $100 \mathrm{~mm}$ collimating lens generate a ring pattern of a selected diameter. Various mirrors are used to scan this pattern onto the surface of a lens mounted on the radial stretcher. The lens refracts the light, which passes through the thin glass panel and onto a CMOS camera (UC480, Thorlabs), which is mounted on a translating stage (UniSlide, Velmex Inc.). This translating stage is computer-controlled and allows the camera to be moved axially, and its position measured. This camera captures monochromatic images (5.2 $\mu \mathrm{m}$ per pixel, image resolution of $1280 \times 1024)$ and automatically stores the position in which they were taken. Figure $2.4 \mathrm{~A}$ describes the system schematically and Figure 2.4B shows a photograph of the actual system.
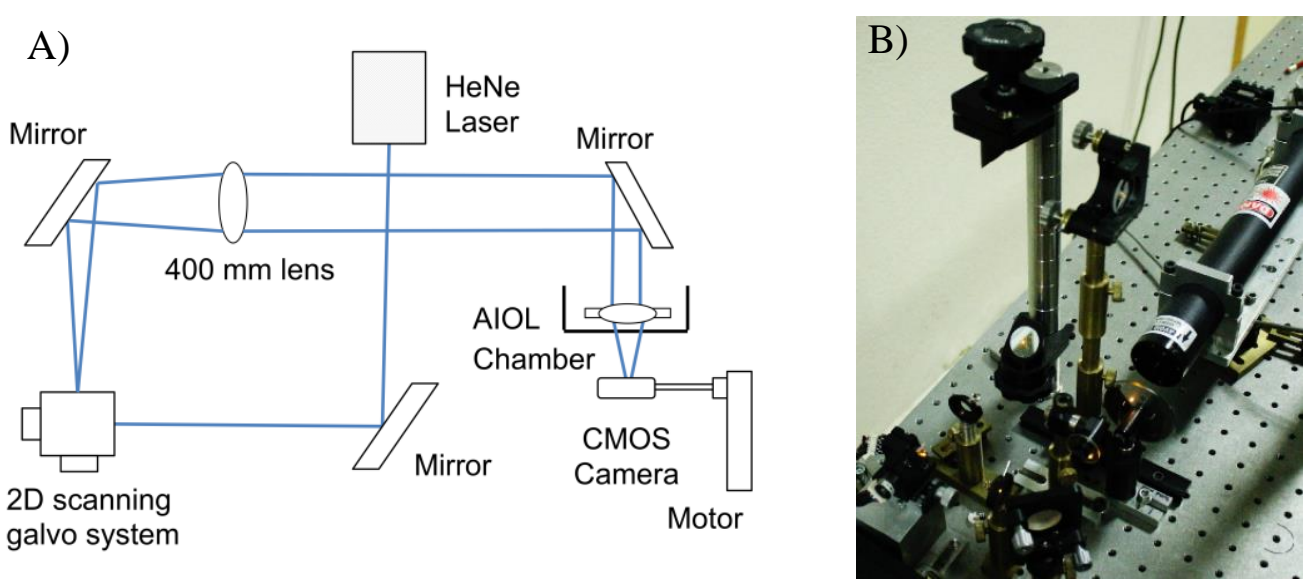

Figure 2.4. A) Schematic of Laser Ray Tracing system. B) Photograph of system.

These images are then analyzed with a custom MATLAB program, which calculates the size of the spot, based on the number of pixels that fall above a threshold of intensity. The position of minimum spot size (and focal length) can be obtained by fitting a parabolic function to the spot size per position.

Validation of the method was achieved by evaluating calibration lenses of a known focal length, capturing a series of images at a rate of 1 image per $0.25 \mathrm{~mm}$ change in stage position for a $30 \mathrm{~mm}$ sweep. The minimum spot size and its corresponding 
position were found for each lens and compared to the known focal lengths with a linear fit. The slope found was 1.06 and the intercept was found to be $28.7 \mathrm{~mm}$, which was close to the estimated distance between the sample and the camera surface at maximum height. This calibration can be seen in Figure 2.5.

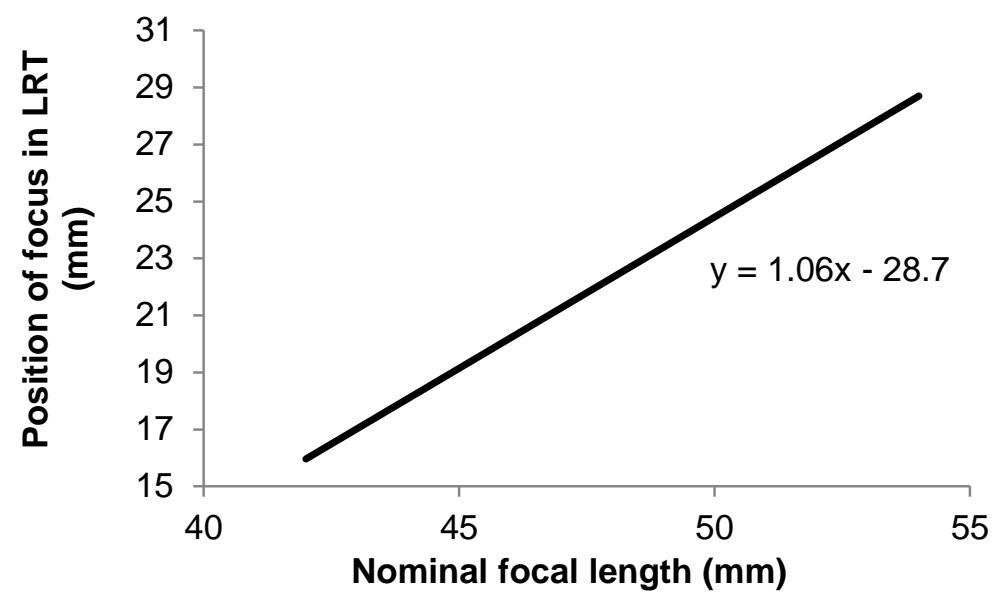

Figure 2.5: Evaluation of calibration lenses in LRT system.

\section{Optical Coherence Tomography}

The LRT system offers a measurement of the change in power, but cannot measure the underlying behavior that produces such a change (surface deformation). In order to study this behavior, a spectral domain OCT system was built for this study. The set-up of the system follows a fiber optics Michelson interferometer configuration (represented in Figure 2.6), and is similar to previous systems developed by the VioBio research group. Its main distinguishing feature is that the system is combined with both LRT system and the radial stretcher.

The system's light source is a superluminescent diode (Superlum SLD-371, Superlum) with a central wavelength of $840 \mathrm{~nm}$ and a bandwidth of $56.2 \mathrm{~nm}$. This wavelength is suitable for use in water and tissues due to deeper penetration. A 90/10 split is used; $90 \%$ of the light goes to the sample and the remainder to the reference mirror. At the sample arm, the light source is collimated and reflects on $\mathrm{X}-\mathrm{Y}$ scanning mirrors (ThorLabs GVS002). The sample arm has a $100 \mathrm{~mm}$ focal length lens (AC254, Thorlabs) anti-reflection coated for infrared light. The sample arm head is mounted on a translation stage, which allows for changing the position of the focus of the lens. 
The light from the sample arm and reference arm then goes through a collimator lens and to the spectrometer, which consists of a holographic Bragg transmission grating (HD 1800 1/mm @ 840 nm, Wasatch Photonics), and a CCD line camera (spL4096$140 \mathrm{~km}$ Basler Sprint, Basler), which records the interference fringes. The OCT system is controlled by a custom-built LabView program. The program controls the galvanometer mirrors, records the interference fringes, and provides a preview for alignment of the sample in two axes. The LabView program saves the data as A-line spectra. The saved data from the measurements is processed using a custom-built Matlab program. The program performs a Fourier transform on each individual spectrum, and compiles the A-lines into an image. The images (B-Scans) are exported for analysis. The entire system can be seen in Figure 2.7 .

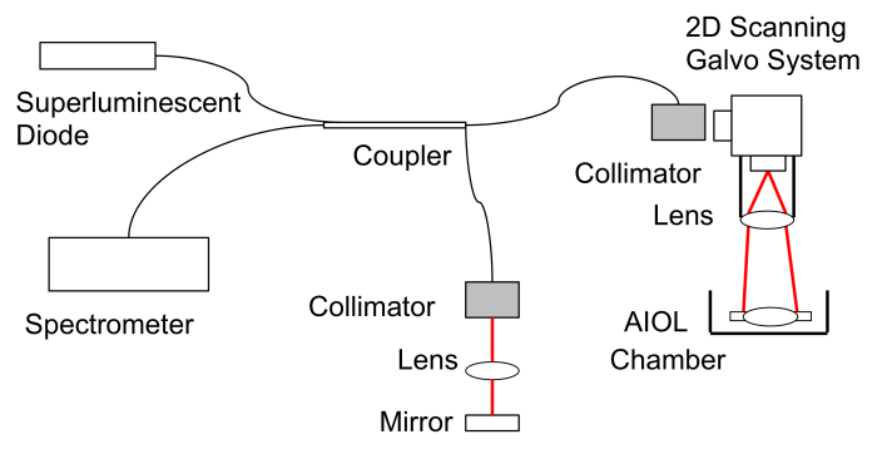

Figure 2.6: Schematic diagram of OCT system.

The axial acquisition rate of the system is $50000 \mathrm{~A}-\mathrm{scans} / \mathrm{s}$, with an axial range of approximately $5 \mathrm{~mm}$ and a theoretical pixel resolution of $5.2 \mu \mathrm{m}$, and a roll-off sensitivity of $-2.4 \mathrm{~dB} / \mathrm{mm}$. The system produces B-scans, cross-sectional images of the AIOL. These images are then processed using custom programs designed by the VioBio group, in order to reconstruct the volume and analyze its surfaces and thickness.

The acquired data is processed to correct for two important sources of distortion: optical and fan distortion. Optical distortion is produced by the refraction that occurs when rays are bent by the surfaces of the media they pass through. In the case of this experimental set-up, light passes through water (as the test is done immersion) and through the first surface of the lens, both of which cause distortion. Fan, or field distortion, is produced by the scanning architecture of the sOCT system. The fan of principal rays of the light source is not flat relative to the optical axis, which generates curved reproductions of 
flat surfaces. The VioBio group has previously designed and validated algorithms to correct for both optical and fan distortion. For fan distortion correction, a calibration plate consisting of an evenly spaced grid (Figure 2.8) was measured in the system and used to estimate the fan distortion. The algorithms are described in more detail in the literature (Ortiz et al., 2010; Ortiz et al., 2009).

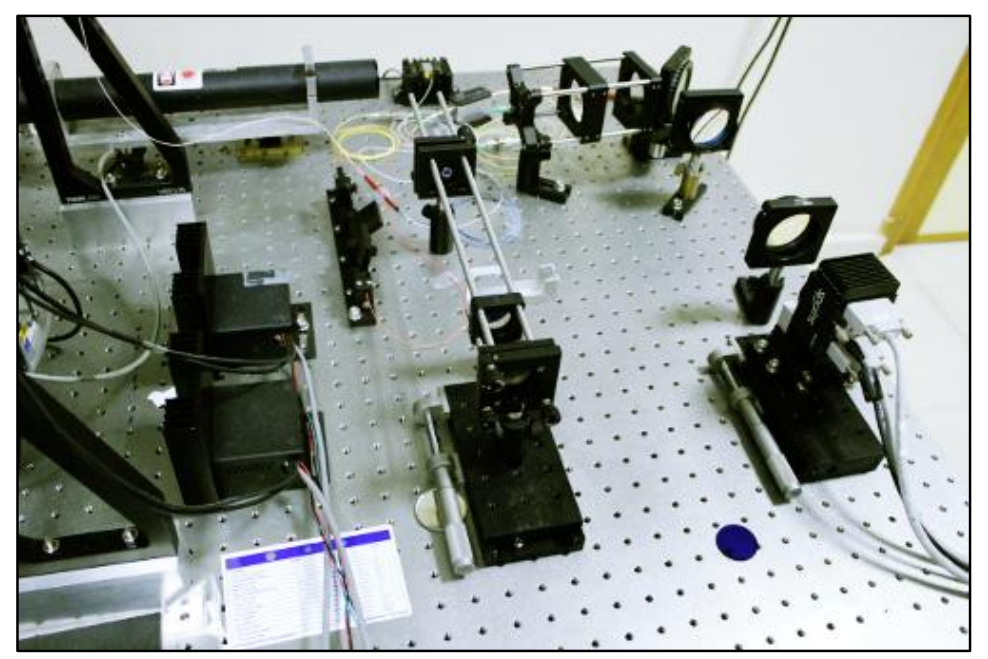

Figure 2.7: OCT component of the accommodation system: reference arm and spectrometer.

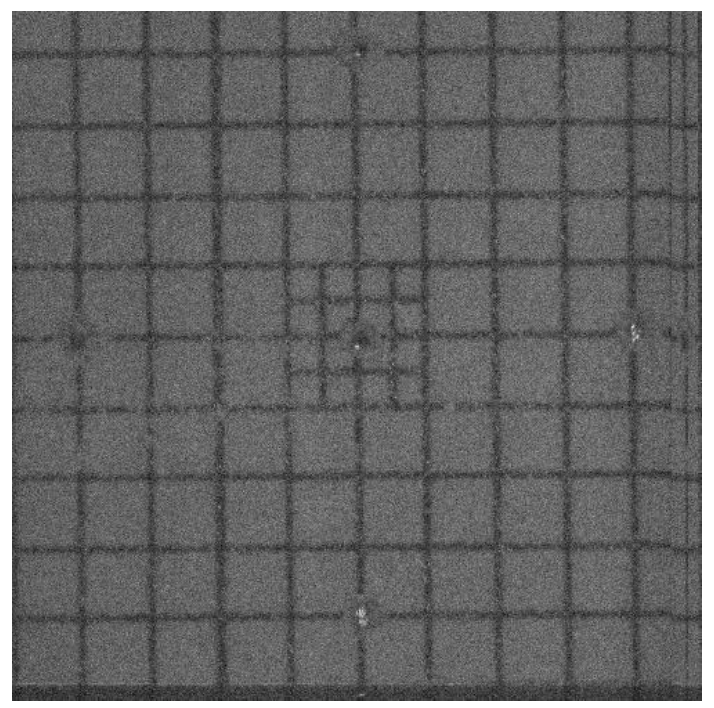

Figure 2.8: Top view of calibration grid retrieved from OCT measurements for fan distortion correction.

\subsubsection{Corvis $\mathbf{S T}$}

The Corvis ST (Oculus, Germany) is a commercial tonometer used in ophthalmology diagnostics. The Corvis ST applies a controlled air pulse onto the subject's cornea, which deforms it axially. The system is combined with a high-speed Scheimpflug imaging camera, which records the cornea's deformation. A slit of light is projected 
onto the eye at $45^{\circ}$ from the optical axis. The eye's horizontal meridian is imaged, and the system generates 140 images during the $30 \mathrm{~ms}$ corneal deformation event. The system then processes the images and quantifies the elevation data from the images, using proprietary parameters that define the cornea's deformation. These parameters are: HC (highest concavity) time, Deformation Amplitude (maximum amplitude at the apex in HC), A1 and A2 (first and second applanation) time and length. These parameters are used by the system to approximate an IOP value for the eye being measured.

Before its commercial release, Oculus provided a prototype of the Corvis ST to the VioBio Lab. This prototype enables the extraction of the quantified elevation data from the system. With this data, the temporal and spatial profiles of the cornea's deformation can be reconstructed. These spatial profiles were used to define and quantify our own relevant parameters.

The air puff was previously quantified at the lab using a pressure sensor to measure the air's temporal variation. This process is described in more detail in the literature (Kling et al., 2014).

\subsubsection{CellScale Ustretch}

The CellScale Ustretch (Cellscale, Waterloo, ON, Canada) is a commercial testile testing device designed for use with biomaterials. The system has two aligned mechanical arms. The first arm is attached to a $5 \mathrm{~N}$ load cell for measuring force. The second arm is attached to an actuator. A sample can be clamped between the two arms; the second arm applies a controlled strain (defined by either a constant displacement or constant force) and the first arm measures the force required to deform the sample.

The device can be used in vertical or horizontal configurations. When in a horizontal configuration, a water bath can be mounted underneath the sample, allowing for the sample to be hydrated and the measurement to be done in immersion conditions. A heating platform below the water bath, and a temperature sensor that runs from the device to the water bath, allow for the water temperature to be controlled and kept constant. The device has a built-in CCD imaging system that allows for the recording of images of the sample during the loading event. Proprietary software allows for a strain 
and stress analysis using these images. These features were not used in the work presented in this thesis.

The system produces raw data in .csv format, with the following outputs: step name, time (s), displacement $(\mathrm{mm})$, size $(\mathrm{mm})$, and force $(\mathrm{N})$. The size and force data can be used alongside measurements of the sample's cross-sectional area to calculate the stress and strain in the sample. The maximum force required to break photochemical bonds can also be obtained from the raw data.

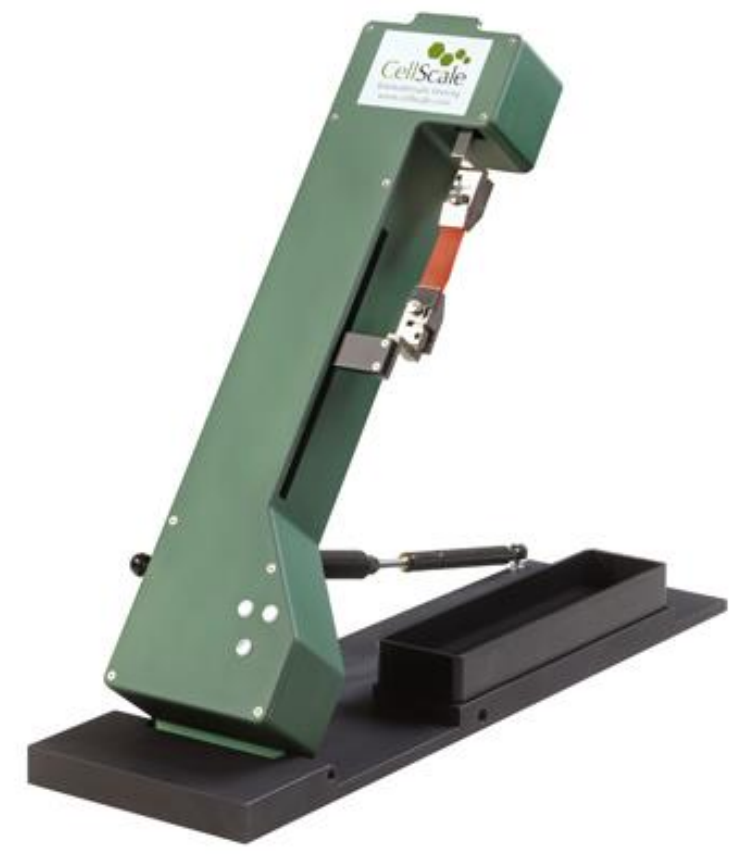

Figure 2.9: Ustretch system used for the work in this thesis.

\subsubsection{Instron 5543A}

The Instron 5543A (Instron Corp, Norwood, MA, USA) is a commercial uniaxial tensile testing system. The system used in this work belongs to the Center of Biomedical Technology of the Universidad Politécnica de Madrid. It is a more robust system than the Ustretch.

This system has a custom-built immersion bath. The bath is made of PMMA and is connected to a water reservoir. The water reservoir has a heating platform and temperature control, allowing the temperature of the liquid on the immersion bath to be set by the user. The Instron 5543A was used to evaluate stress-strain relationships of polymers. The testing chamber can be seen in Figure 2.10. 


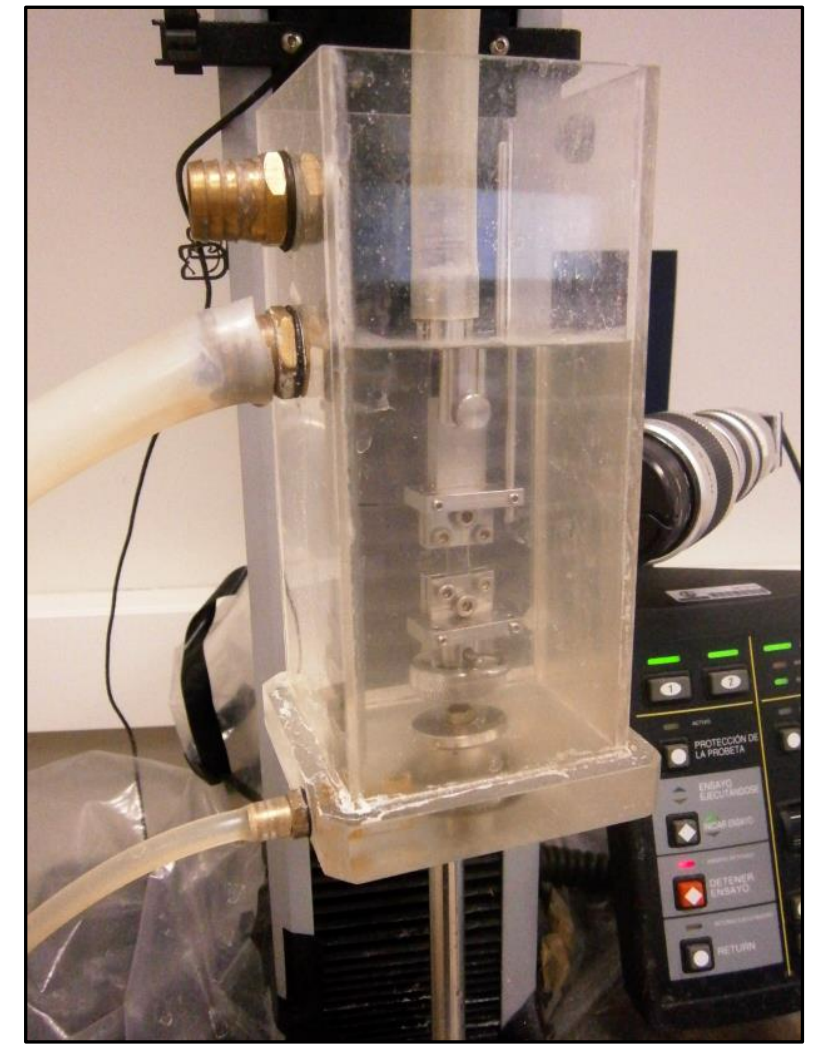

Figure 2.10: Instron 5543A. Clamps are within custom-built immersion bath.

\subsubsection{Brillouin Confocal Microscope}

Measurements taken with a Brillouin confocal microscope were used in this thesis and provided by researchers from the University of Maryland. The confocal microscope and its technical details are described in detail in the literature (Scarcelli et al., 2011; Scarcelli \& Yun, 2007). Briefly, a frequency-doubled Nd-YAG laser (Torus, Laser Quantum, UK) with a wavelength of $532 \mathrm{~nm}$ is focused by an objective lens onto a sample. The scattered light is collected from the sample by a single-mode optical fiber and measured by a spectrometer. The main technical feature of this Brilloun confocal microscope, is the incorporation of a virtually-imaged phased array for the spectrometer, allowing for parallel imaging and dramatically reducing the spectrum acquisition time to $0.1 \mathrm{~s}$. The sample is mounted on a 3 -axis translation stage and shifted in constant intervals, allowing for the measurement of a spatial profile of the sample. MATLAB is then used for spectral analysis and a custom algorithm is used to produce the Brillouin shift and magnitude as a function of position.

Reconstructed spatial profiles of lenses were provided by the University of Maryland and used for modeling and finite element analysis. 


\subsection{Software}

\subsubsection{ANSYS Finite Element Software}

The numerical models used in this work were built using the finite element software ANSYS 16.0 (ANSYS, Inc. Canonburg, PA, USA). ANSYS 16.0 has two main finite element platforms: Mechanical APDL and Workbench. Both were used to develop the models in this work.

Both Mechanical APDL and Workbench use the same finite element analysis code. APDL stands for ANSYS Parametric Design Language, the system's design language that is based on FORTRAN. Mechanical APDL has a GUI, but is largely codingoriented. It is suitable for applications where substantial pre- and post-processing is required. ANSYS Workbench is design-oriented; besides its advanced GUI, it comes with its own CAD platform, DesignModeler. It is suitable for applications where the pre- and post-processing is limited, and either complex geometries or substantial changes in initial geometry (for parametric optimization) are expected.

In this thesis, Finite Element models of the cornea, crystalline lens, and accommodating intraocular lens were created. Each model was compared with experimental data, either as part of an inverse modeling process or for validation of experimental results. The structure of the model was determined by the data: Cornea and crystalline lens models were 2-dimensional, and the accommodating IOL model was 3-dimensional.

An overview of the process of developing a finite element model is presented, using a 2-dimensional axisymmetric cornea model and 2-dimensional crystalline lens model as visual references.

\section{Geometry}

The process for building a numerical model is similar in both platforms. The first step is building the geometry. Workbench's DesignModeler GUI (Figure 2.11) allows for parametric geometry creation; APDL creates geometries of fixed length. Both platforms offer the same level of control in this step. The next step is to create materials. Material properties are introduced for a selection of standard material constitutive models (linear elastic, hyperelastic, viscoelastic, etc.). A material must be assigned to each body in the geometry. 


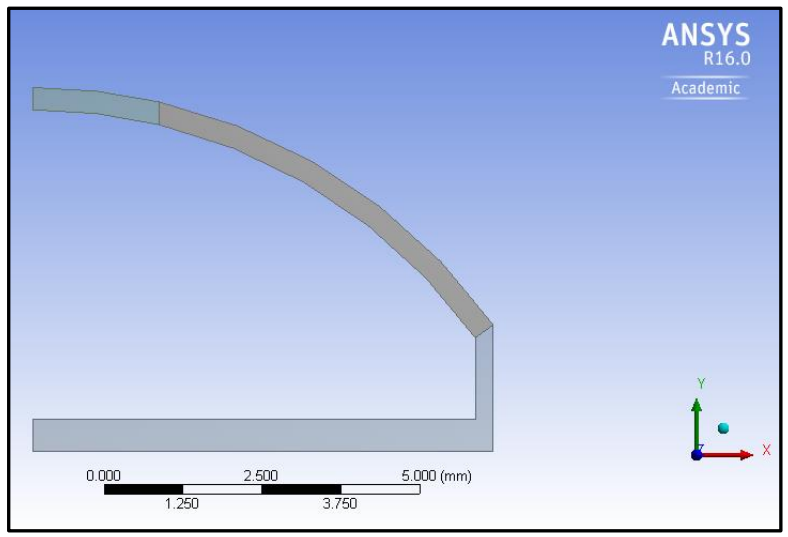

Figure 2.11: ANSYS Workbench DesignModeler geometry of a 2D axisymmetric cornea.

\section{Mesh}

The second step is to create the finite element mesh (Figure 2.12). A material must be assigned to each body in the geometry. The size of the mesh can be controlled by selecting edge length or element size for specific volumes, areas, or lines of the bodies to be meshed, and can be refined along sections of interest.

Elements must be selected and assigned to the geometries to be meshed. Element types are selected on the basis of problem type, analysis type, and loading conditions.

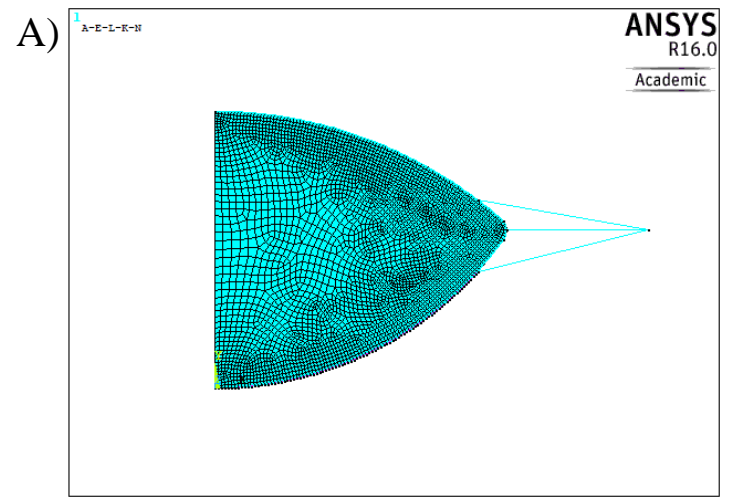

B)

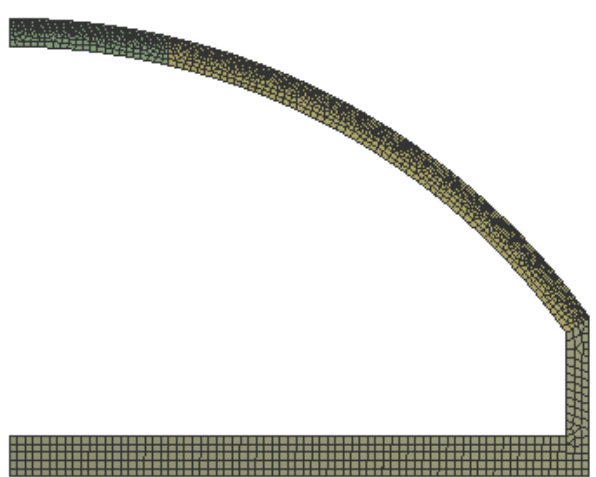

Figure 2.12: Meshing in A) ANSYS Mechanical APDL B) ANSYS Workbench. The APDL model is a 2D axisymmetric model of the crystalline lens; the Workbench model is the axisymmetric cornea.

Mechanical APDL allows for the selection of elements to be used in the model and for setting their individual properties, such as element behavior, formulation, and technology for solid elements (Figure 2.13). Workbench can select elements automatically or the user can select them. This completes the geometric and mesh model. 


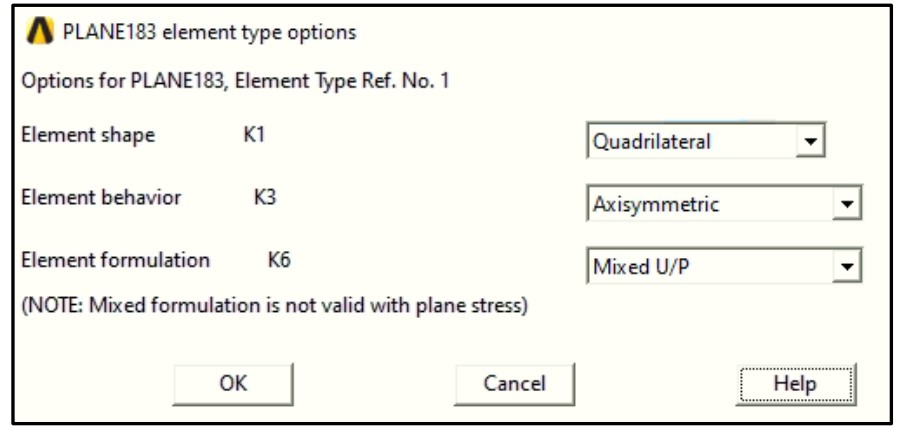

Figure 2.13: Element options for PLANE183

\section{Solution}

The third step is to define the solution. Both platforms allow a selection of the analysis type, the number of load steps and substeps, and the output to be saved. APDL also allows for the selection of the equation solver and selection of non-linear options. Then the user defines the boundary conditions in the problem: displacement, force, moment, pressure, or temperature loads applied to surfaces or lines, symmetries, and fixed regions (Figure 2.14). With this information, a solution can be computed. Depending on the number of elements and the number of substeps and load-steps this process can take anywhere from seconds to hours.
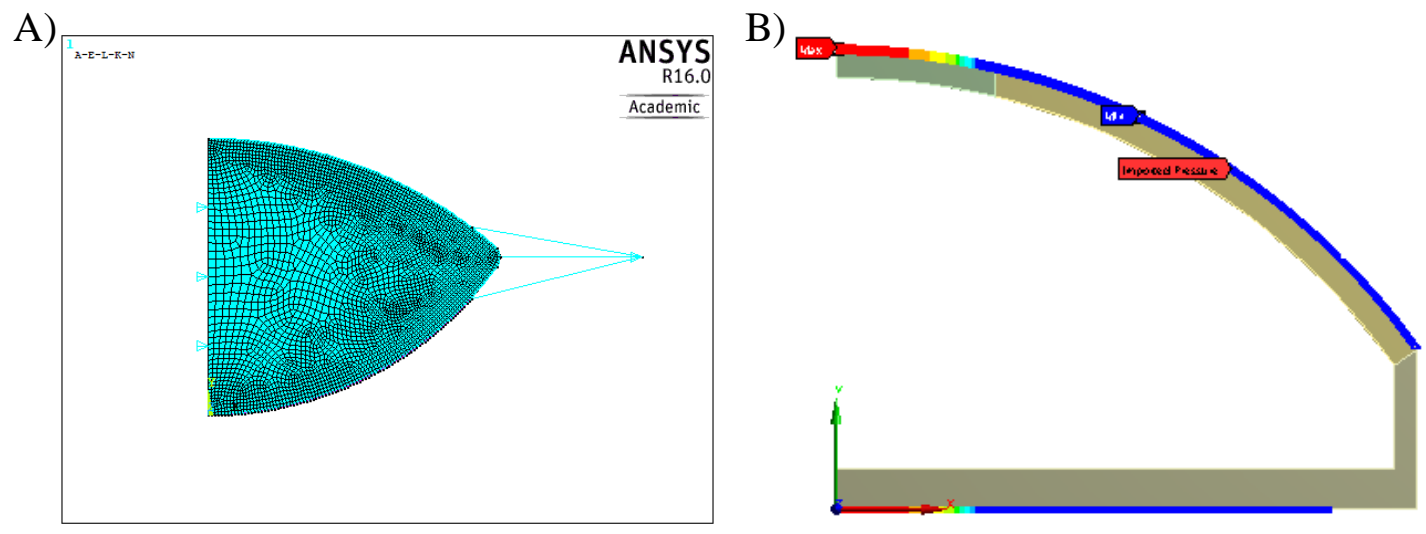

Figure 2.14: Boundary conditions and loads in A) Mechanical APDL B) ANSYS Workbench.

\section{Post-Processing}

The fourth step is post-processing of the solution. Both systems have built-in postprocessing and analysis for the most relevant mechanical data: displacement, stress, strain (elastic, plastic, etc.). Custom post-processing can be generated through the GUI if the information required is accessible through it. If not, APDL can be used to extract solution data from individual nodes and elements, and calculations can be done with 
this data. The data can also be exported as a matrix. APDL codes can be inserted within the Workbench model and used to process data. The main limitation of this approach is that Workbench can only display single variables, or export data. Therefore, the approach is not suitable for displaying matrices or tables of data. Figure 2.15 shows displacement loads in Mechanical APDL and Workbench.

Workbench was used to create models of the cornea and accommodating intraocular lenses, as these were more suited to a design-oriented GUI. Mechanical APDL was used to create models of the crystalline lens, as these required an important and laborious pre-processing operation as well as importing data into the coding environment.
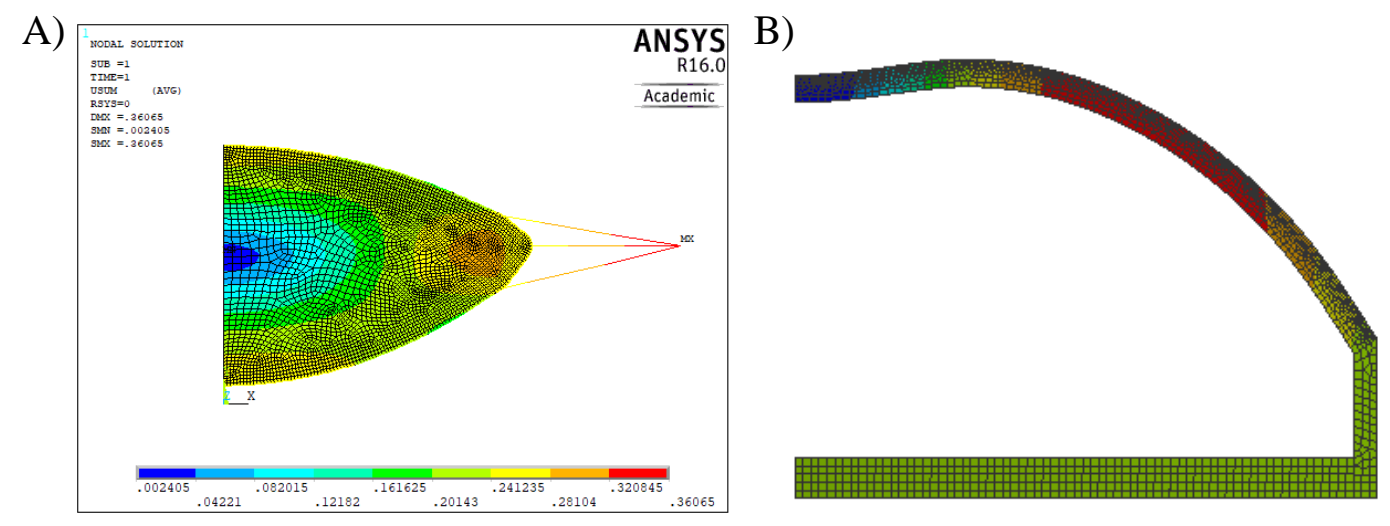

Figure 2.15: Displacement loads in A) Mechanical APDL B) Ansys Workbench.

\subsubsection{Inverse Modeling}

The finite element platforms were used as part of inverse modeling procedures. A schematic of these procedures is presented in Figure 2.16.

The finite element models were built following the procedure described in 2.2.1. The coefficients defining the mechanical properties of the materials were set as variables. Experimental data on lens displacement and load/boundary condition was retrieved experimentally. This data was used to generate a merit function to be minimized.

A code or algorithm was implemented to evaluate the finite element model for a given variable value, retrieve simulation results, import experimental data, evaluate the merit function, and then minimize the computed value of the function. This process was implemented using ANSYS' optimization module in one case, and Python programming language in another. 


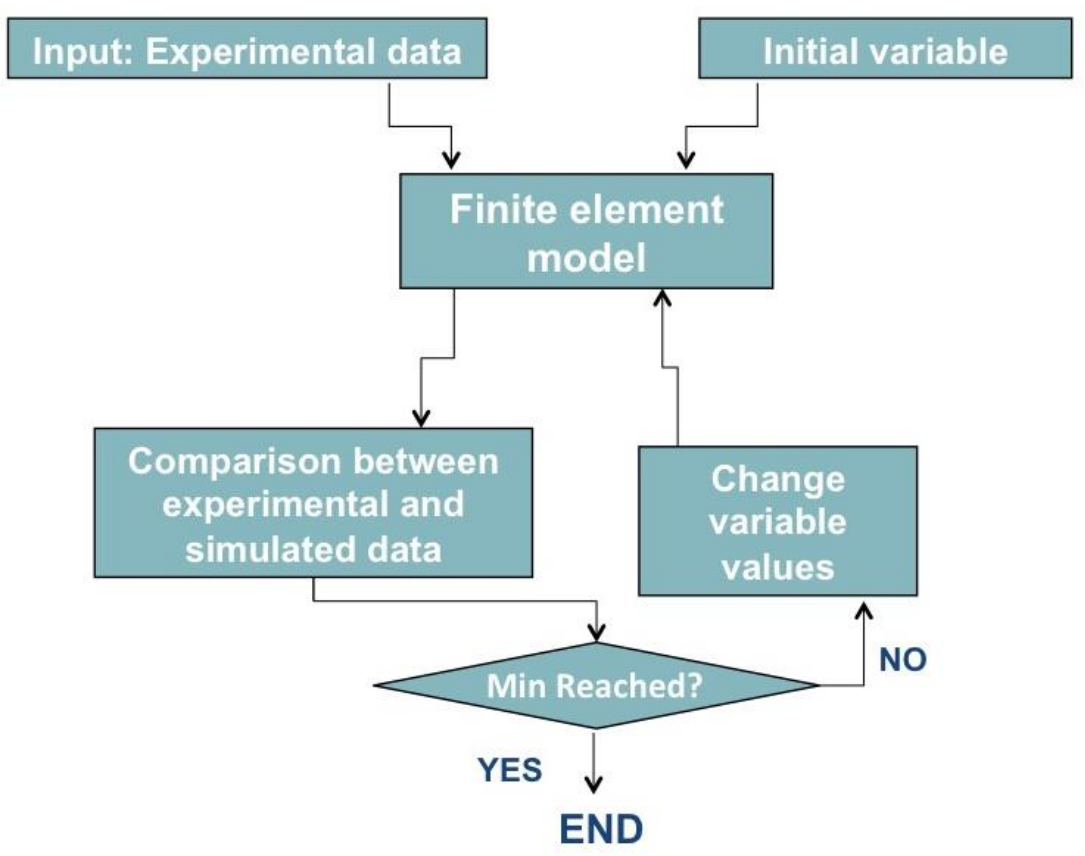

Figure 2.16: Schematic of inverse modeling procedures

\subsubsection{ZEMAX}

ZEMAX is a ray tracing software that allows for modeling of the propagation of rays through optical systems. Optical systems in ZEMAX are defined by surfaces, each with parameters controlling their curvature, thickness (or distance to next surface), refractive index, and diameter. The number of rays to be traced, their angle, and wavelength can also be defined. The software can provide output in optical terms (optical path difference, ray fan, field curvature, modulation transfer function) and system data (focal lengths, paraxial image height, etc.)

ZEMAX provides an extensive number of surface options. The Standard option consists of defining the surface via a radius and conic value, using the following equation:

$$
z=\frac{c r^{2}}{1+\sqrt{1-(1+k) c^{2} r^{2}}}
$$

Where $\mathrm{c}$ is the curvature (the reciprocal of the radius), $\mathrm{r}$ is the radial coordinate, and $\mathrm{k}$ is the conic constant. This surface type is used for the majority of modeling in this thesis.

Optical surfaces can also be defined by Zernike coefficients. In this work, the Zernike Standard Sag surface was used for this analysis. This surface is defined as: 


$$
\sum_{i=1}^{N} A_{i} Z_{i}(\rho, \varphi)
$$

Where $\mathrm{N}$ is the number of Zernike coefficients, $A i$ is the coefficient on the $i^{\text {th }}$ Zernike standard polynomial ("ZEMAX Optical Design Program User's Guide," 2005).

\subsection{Applications of the technology in the thesis}

The hardware and software presented in this chapter was used in the following way:

- ANSYS Finite Element Software was used to create models of the cornea (Chapter 3), crystalline lens (Chapter 5), and an accommodating IOL (Chapter 6). The cornea and crystalline lens models were combined with the inverse modeling method.

- Instron 5543A and CellScale Ustretch devices were used to evaluate the mechanical properties of corneal strips (Chapter 3) and polymers (Chapter 6), as well as the bonding strength of silk and corneal strips (Chapter 4).

- Corvis ST was used to obtain air pulse measurements of corneas (Chapter 3).

- The mechanical stretcher, OCT, and LRT systems were used to evaluate the performance of an accommodating IOL (Chapter 6).

- Brillouin Confocal Microscopy was used to measure the spatial mechanical profile of crystalline lenses (Chapter 5).

For both elements of the anterior segment (cornea and crystalline lens), a finite element inverse modeling method was used to evaluate objective mechanical properties from experimental data, and a proposed correction to a condition (wound healing, presbyopia) was evaluated. 


\section{Material properties of the cornea from air puff deformation and numerical simulations}

\subsection{Introduction}

The cornea is under loading from the eye's internal pressure (intraocular pressure, or IOP), and changes in its mechanical properties can cause a number of pathologies (corneal ectatic disorders), described in Section 1.2.2.

Most experimental studies on the mechanical properties of the cornea are ex vivo, destructive measurement of corneal strips or whole cornea, using standard mechanical testing. These are limited by the effect of post-mortem conditions on corneal mechanical properties, and can only yield information for research, and not diagnostic purposes, as they are invasive. For diagnostic purposes and for in vivo research, noninvasive methods are required.

A common approach has been to use tonometry to apply a characterized, consistent air pulse load to a cornea, use an imaging system to capture the cornea's response to this load, and use statistical analysis to separate and quantify the influence of various parameters (corneal mechanics, geometry, IOP).

Another possibility is to use finite element analysis. In traditional FEA, the problem is defined by geometry, constitutive stress-strain relationships, and boundary conditions or loads. The solution of the model is the deformed geometry after loading and its local stress and strain values. Tonometry and imaging provide the undeformed and deformed geometry, and some (or all) boundary conditions, but not the constitutive stress-strain models. An inverse modeling approach can be used in this case, where an algorithm solves the finite element model for a variable constitutive model (or boundary condition), compares the FE model's output to an expected output via a merit function, and finds the constitutive model that minimizes the merit function. This method has been used previously to study corneal biomechanical properties, with hyperelastic and viscoelastic material models. 
This work is a validation of this method. In this work mechanical properties retrieved from an inverse modeling method on model polymer corneas and porcine corneas are compared to mechanical properties retrieved from uniaxial testing.

\subsection{Protocols for corneal mechanical characterization}

\subsubsection{Model Corneas}

Three types of model corneas (MCs) were evaluated. The MCs were manufactured by a contact lens manufacturer (Mark'ennovy, Madrid, Spain). The three MCs were manufactured out of commercially available, characterized hydrophilic properties. The three MCs will be referred to by the initial of their brand names: Gentle (G), Saphir (S), and Quattro (Q). The nominal properties of the MCs are described in Table 3.1.

\begin{tabular}{|cccc|}
\hline Material name & Gentle (G) & Saphir (S) & Quattro (Q) \\
Young's Modulus (MPa) & 0.16 & 0.3 & 0.38 \\
Water content \% & $79+-2$ & $75+-1$ & $49+-2$ \\
Thickness & 350 & 450 & 550 \\
Anterior Radius of Curvature & 8.60 & 8.60 & 8.60 \\
Posterior Radius of Curvature & 8.95 & 9.05 & 9.15 \\
\hline
\end{tabular}

Table 3.1: Nominal properties of model corneas

\subsubsection{Porcine Corneas}

Two porcine eyes (PCs) were used for this study. Measurements were done 4 hours post-mortem. The eyes were kept refrigerated before sample preparations. Corneas ( $\mathrm{P} 1$, P2) were excised from the ocular globe for measurement purposes. The central corneal thickness was measured with the Corvis ST and the values were $724 \mu \mathrm{m}$ for P1 and 625 $\mu \mathrm{m}$ for $\mathrm{P} 2$.

\subsubsection{Corvis ST Tonometry}

The Corvis ST system (described in 2.1.2) was used to deform the corneas and record the deformation event. A plastic artificial eye chamber was used to house and restrain the MCs and PCs. The chamber was filled with saline solution, using a water column to set the baseline pressure at $15 \mathrm{~mm} \mathrm{Hg}$. The system is represented in Figure 3.1.

The MCs were previously stained in fluorescein $(0.1 \%)$ for $30 \mathrm{~s}$ in order to improve their detection. Both the MCs and the PCs were sprayed with water every 60 seconds to maintain their hydration levels. 


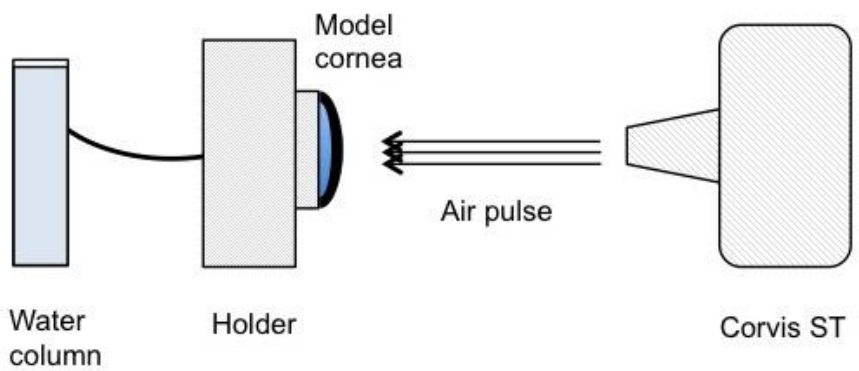

Figure 3.1: Schematic of experimental set-up

The eye chamber was placed in front of the Corvis ST and its imaging system was used to properly align the corneas and maintain a consistent distance. The system is set up to capture 140 cross-sectional images of the sample during the $30 \mathrm{~ms}$ deformation with a resolution of $640 \times 480$ pixels. The system processes the images and provides quantification of key deformation parameters.

After conducting the measurements, the raw data from the Corvis ST was extracted and used to build spatial and temporal profiles. The spatial profile is the displacement at all positions for the maximum deformation time (Time to Highest Concavity). The temporal profile is the displacement of the central node of the MC over time (Central Deformation Amplitude, or DA). Additionally, PDA (Peripheral Deformation Amplitude), which measures the displacement of a point $2 \mathrm{~mm}$ from the apex, was extracted and used to derive the Central-Peripheral Deformation Ratio, or CPR (DA/PDA).

\subsubsection{Uniaxial Tensile Testing}

Tensile tests were done in order to obtain an independent experimental measurement of mechanical properties of MCs and PCs.

Testing of MC materials was done at the Centro de Tecnología Biomedica in the Universidad Politécnica de Madrid using an Instron 5543A device (described in Section 2.1.4) with a $10 \mathrm{~g}$ load cell. Sample strips were obtained from the MCs. Tests were conducted in immersion $\left(\mathrm{H}_{2} \mathrm{O}\right)$ at a temperature of $20^{\circ} \mathrm{C}$. A measurement was done without a sample in order to quantify the force that the immersion fluid exerts on the measuring arm and this data was incorporated into the post-processing to remove the effect of the buoyant force. The dimensions of each sample of material were measured before the experiment. The dimensions were approximately $16 \times 3 \times 0.55 \mathrm{~mm}$ for all 
samples. The strips were clamped and mounted, with a consistent distance of $8 \mathrm{~mm}$ between the clamps. Three samples of each material were measured, at a rate of $1 \mathrm{~mm} / \mathrm{s}$.

Testing of porcine strips was done at the Institute of Optics using a CellScale Ustretch device (described in Section 2.1.3). The strips were cut from the same porcine corneas that were evaluated in the air puff, with an average length and width of 10 and $5 \mathrm{~mm}$ respectively. The measurements were done in immersion $\left(\mathrm{H}_{2} \mathrm{O}\right)$ at room temperature $\left(25^{\circ} \mathrm{C}\right)$, at a rate of $0.1 \mathrm{~mm} / \mathrm{s}$.

\subsubsection{Finite Element Model}

A finite element model of the experimental set-up was developed. The model follows a similar design procedure as previous works from our research group and was built using ANSYS Workbench 14 (described in Section 2.2.1).

\section{Geometry}

The geometries of the MCs and PCs were modeled from the nominal data and from the spatial profile obtained from the Corvis ST at $t=0$. The lens holder was modeled as a rectangular hollow chamber. The model is an axisymmetric cross-section of the cornea and holder.

The cornea was separated into two regions: a central region of $3 \mathrm{~mm}$ diameter, and the remainder of the cornea. This facilitated the selection of the node $1.5 \mathrm{~mm}$ away from the apex, used to calculate the PDA and CPR.

\section{Elements \& Mesh}

Figure 3.2 shows a representation of the model. The cornea and the holder were assigned PLANE182 4-node elements for compatibility with axisymmetric conditions. Both geometries were meshed with an element edge length of $0.1 \mathrm{~mm}$.

An APDL code was implemented to mesh HSFLD241 elements (2-d hydrostatic fluid elements) into the hollow chamber. These elements are automatically centered along a pressure node residing on the axisymmetry axis.

\section{Material Properties}

A linear elastic constitutive model was used for the holder, with a Young's Modulus value of $1500 \mathrm{MPa}$ and poisson's ratio of 0.49 . 
The 2-term Mooney Rivlin model allows for modeling of simple non-linear material behavior (exponentially increasing stress) and is suitable for the limited strain range expected from an air pulse deformation event.

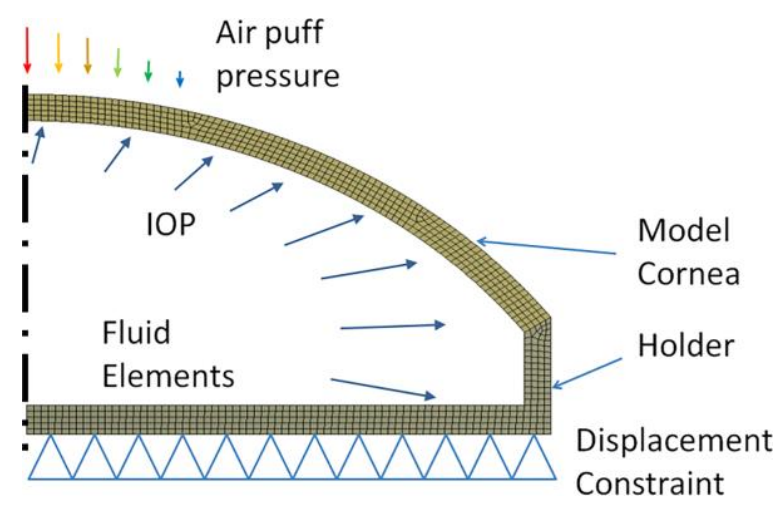

Figure 3.2: Finite element model of MC under loading

\section{Solution}

Transient analysis was selected, with axisymmetric geometry and large displacement. 141 steps were assigned to the model. The first 140 correspond to the 140 steps obtained from the Corvis ST and an additional load step was added to return to initial, pre-loaded state. The time assigned to each load step was selected on the basis of the time recorded by the Corvis ST.

Bonded contact was established between the cornea and the rectangular holder and a fixed displacement was assigned to the lowermost edge of the holder.

The IOP boundary condition ( $15 \mathrm{~mm} \mathrm{Hg}$ ) was applied on the pressure node connecting the HSFLD241 elements.

The air pulse was applied as a boundary condition. The spatial and temporal magnitudes of the air pulse have been previously characterized in our laboratory using a pressure sensor that captures the spatial and temporal profile on a flat surface, and simulating the corresponding flow onto a spherical surface such as the cornea. (Kling et al., 2014). This modified spatial distribution is imported into ANSYS as an Imported Load, and the temporal distribution is applied as a scaling factor to each load step. 


\section{Post-Processing}

Nodes are defined at the central apex and $1.5 \mathrm{~mm}$ away from the apex in order to evaluate the DA and the CPR. This data is exported to a comma separated values (csv) file.

\subsubsection{Inverse Modeling}

An inverse modeling procedure is used to retrieve the material properties of the MCs and PCs from the finite element model. The type of procedure is described in Section 2.2.2.

A Python code was implemented that generated input data $\left(\mathrm{C}_{10}\right.$ and $\mathrm{C}_{01}$ parameter values), ran the model in ANSYS Workbench using the pre-generated input data, retrieved the exported output (csv files with spatial profiles), and evaluated the output against the experimentally determined spatial profiles. The merit function was defined as:

$$
\Phi=\left|D A_{m}-D A_{s i m}\right|+\frac{\left|C P R_{m}-C P R_{\text {sim }}\right|}{3}
$$

A built-in adaptive multiple-objective optimization was used. It combines a Kriging surface response surface and a genetic algorithm.

The final values for the constitutive models of each material (C10 and $\mathrm{C} 01)$ were used to generate a stress-strain curve in uniaxial boundary conditions and the results were compared to the experimental stress-strain curves obtained for the same conditions.

\subsection{Mechanical behavior of corneas and model materials}

\subsubsection{Material properties}

Figure 3.3A shows the values of the constitutive model parameters retrieved from the inverse modeling. The variability in the retrieved parameters for MCs is low, less than $7 \%$ for $\mathrm{C}_{10}$ and $13 \%$ for $\mathrm{C}_{01}$. The variability was higher in the parameters for PCs, $30 \%$ for $\mathrm{C}_{10}$ and $33 \%$ for $\mathrm{C}_{01}$.

The two parameters in the Mooney-Rivlin constitutive model do not have a physical significance. In order to quantify the differences in stiffness of the materials, an equivalent Young's Modulus was calculated from the lower strain region $(\mathrm{e}<0.15$ for 
MCs, e>0.06 for PCs). The results are shown in Figure 3.3B. The trends in equivalent Young's modulus correspond to the trends expected from the documentation provided by the materials manufacturer, if not the magnitude of the modulus (likely due to different approaches in uniaxial testing and post-processing). For the MCs, the stressstrain curve is mostly linear in this strain range. The porcine corneal strips show greater non-linearity; as such, the equivalent Young's Modulus is a less accurate

A) approximation.

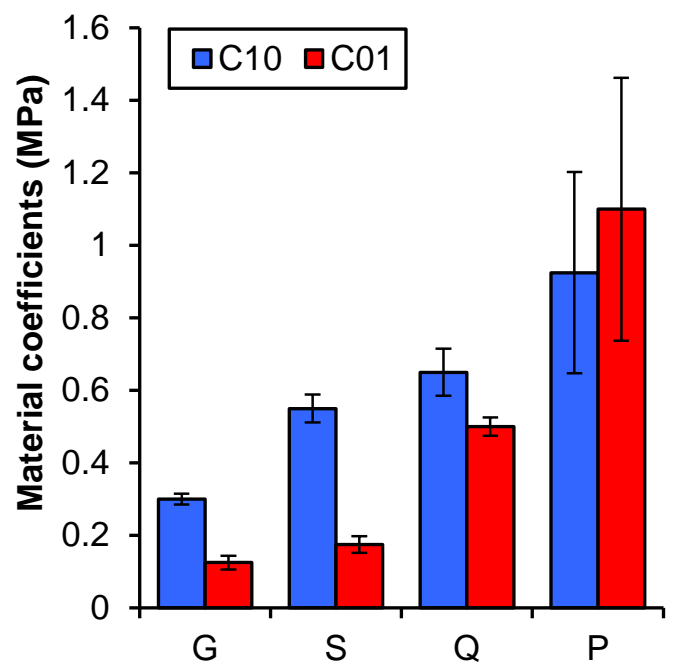

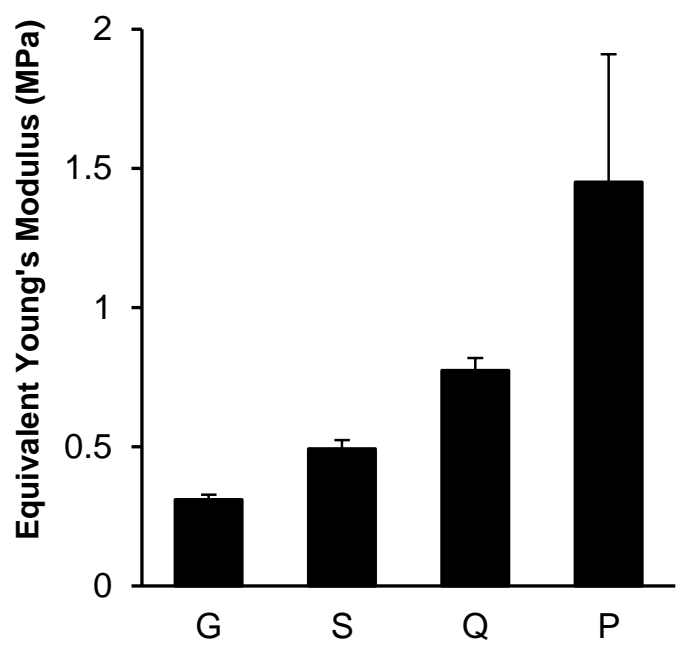

Figure 3.3: A) Material coefficients $\left(\mathrm{C}_{10}, \mathrm{C}_{01}\right)$ retrieved from inverse modelling process. B) Equivalent Young's Modulus of materials at lower strain rates. $\mathrm{G}=\mathrm{Gentle}, \mathrm{S}=$ Saphir, $\mathrm{Q}=\mathrm{Quattro}, \mathrm{P}=$ corneal strips.

The uniaxial response of the materials is shown in Figure 3.4. The response is calculated from the average coefficients of the constitutive model of each material evaluated. Of note is the increased divergence between experimental and simulated stress response with increasing strain for MCs. The numerical model of air puff deformation has maximum strain values of $0.20-0.30$; as such, the stress response for higher strain is not accurately modeled in the reconstruction. The divergence is more noticeable in the stiffer materials (where maximum strain in the simulation was lower). In the strain range of $0-0.4$, the average correspondence between simulated and experimental stress-strain curves was $95.15 \%$ for $\mathrm{G}, 93.52 \%$ for $\mathrm{S}$, and $95.5 \%$ for $\mathrm{Q}$.

\subsubsection{Deformation profiles}

Figure 3.5 shows the experimental and simulated DA and CPR parameters. The deformation amplitude is seen to decrease when the thickness of the cornea increases. A similar trend is observed in the CPR parameter. The differences between experimental 
and simulated CPR parameter are larger, as expected from the merit function weighting the central node.

A)

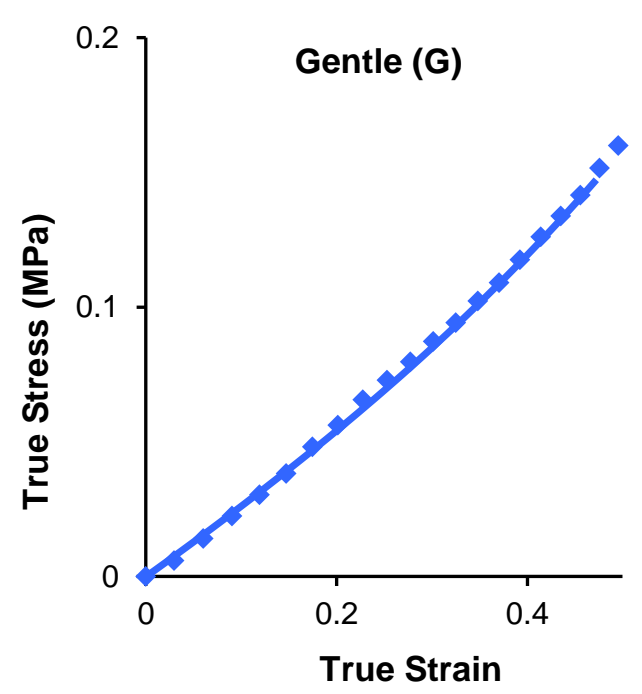

C)

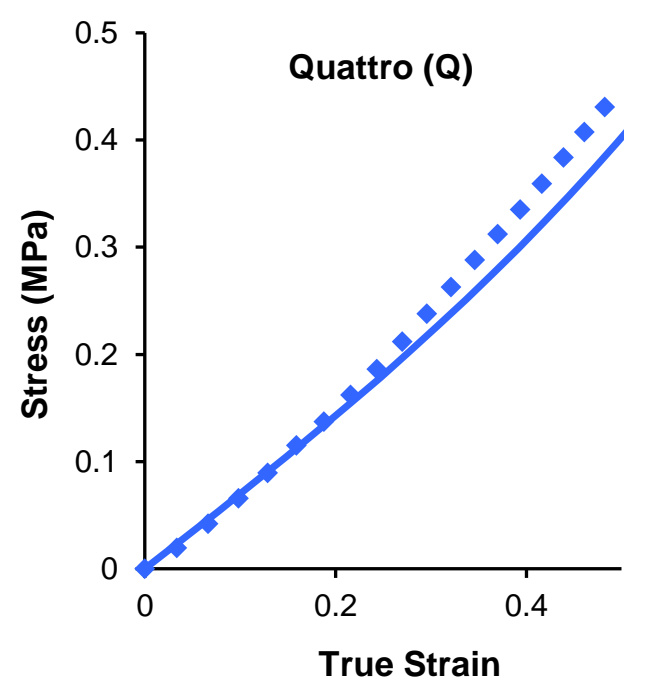

B)

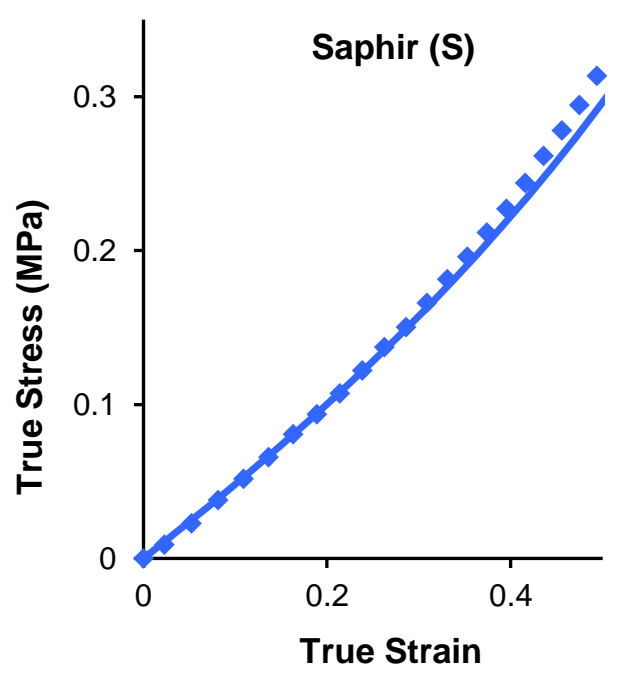

D)

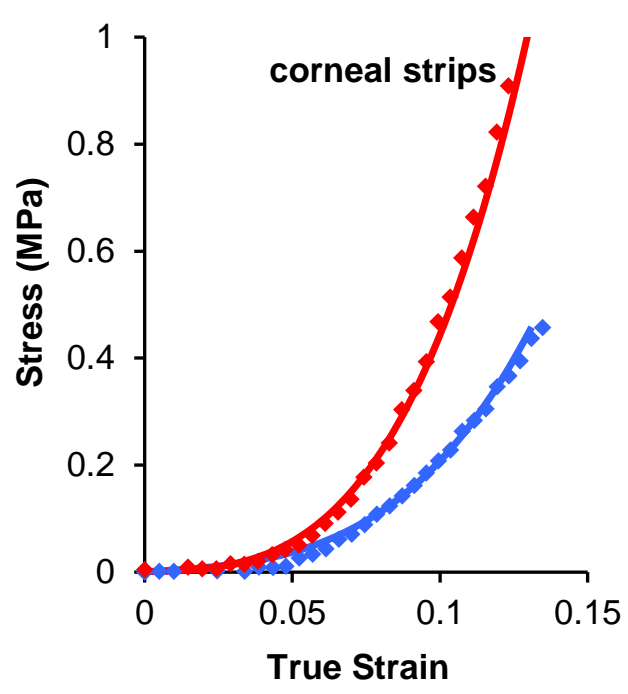

- 2 (exp)

Figure 3.4: Reconstructed uniaxial response vs. experimental uniaxial response for polymers and corneal strips. For corneal strips, results of strip 1 and strip 2 are represented. 
A)

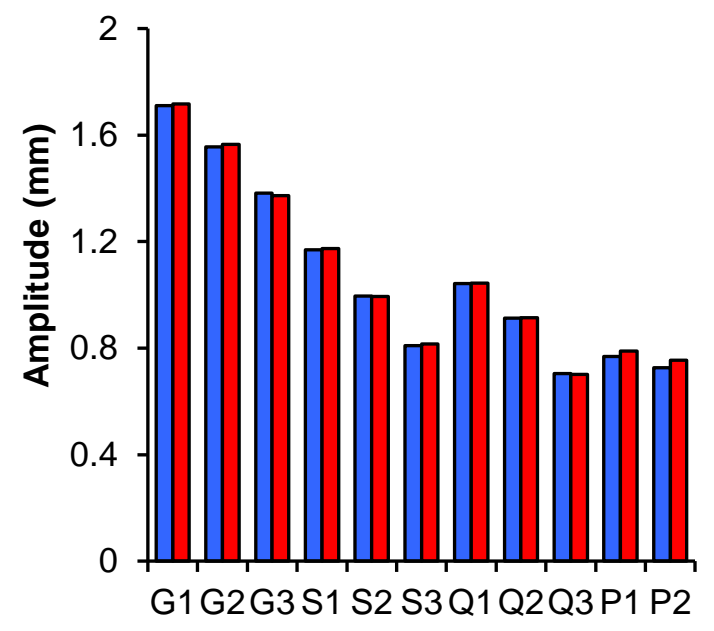

$$
\text { 口DA (sim) QDA (exp) }
$$

B)

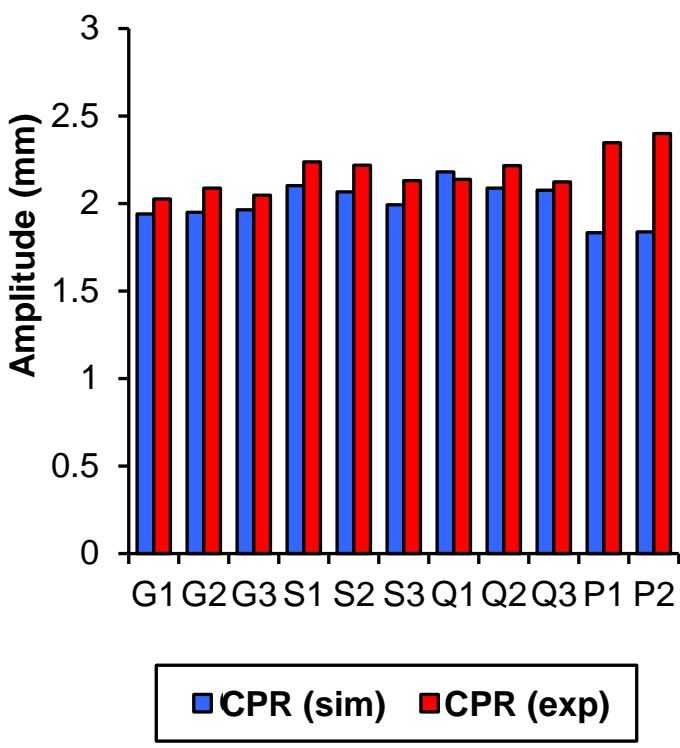

Figure 3.5: Experimental and simulated deformation parameters for all corneas tested. G1-3=Gentle trials 1 through 3; S1-3=Saphir trials 1 through 3; Q1-3=Quattro trials 1 through 3; P1-2=corneal strips.

Figure 3.6 shows the simulated and experimental spatial (at THC) and temporal profiles for all the corneas tested. For the model corneas, the average RMS difference between simulated and experimental profiles was $0.04 \mathrm{~mm}$ for spatial and $0.08 \mathrm{~mm}$ for temporal profiles. For the porcine corneas, the RMS difference was $0.08 \mathrm{~mm}$ for spatial and 0.14 $\mathrm{mm}$ for temporal profiles. The simulated temporal profiles were found to be less smooth than the experimental profiles. This is a result of the characterization of the air pulse. The larger difference in temporal profiles in porcine corneas relative to model corneas is likely due to the lack of a time-dependent constitutive model. 
A)

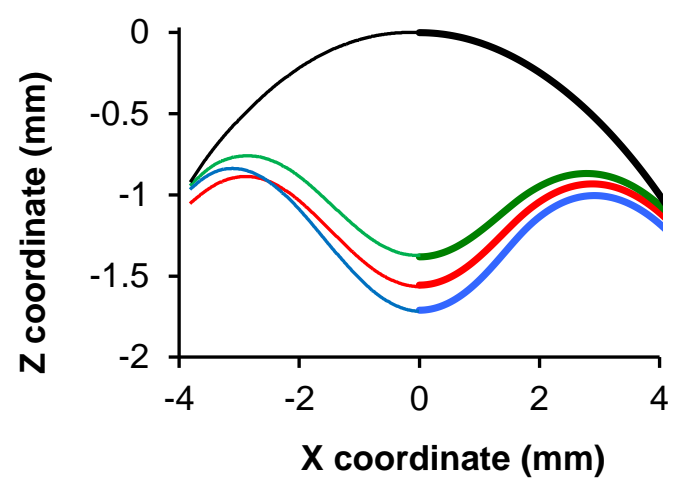

B)

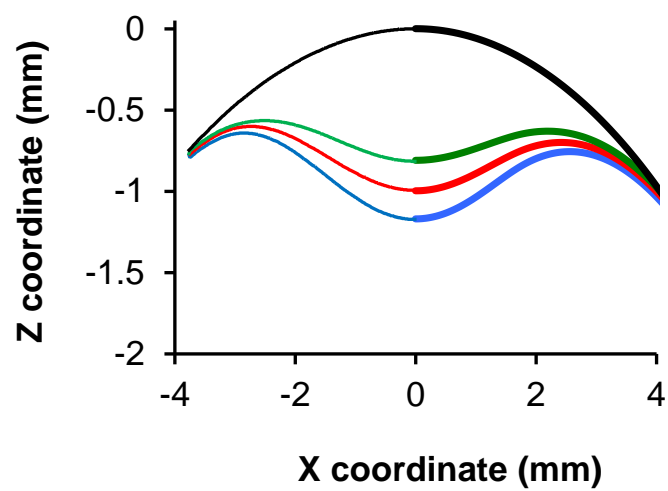

C)

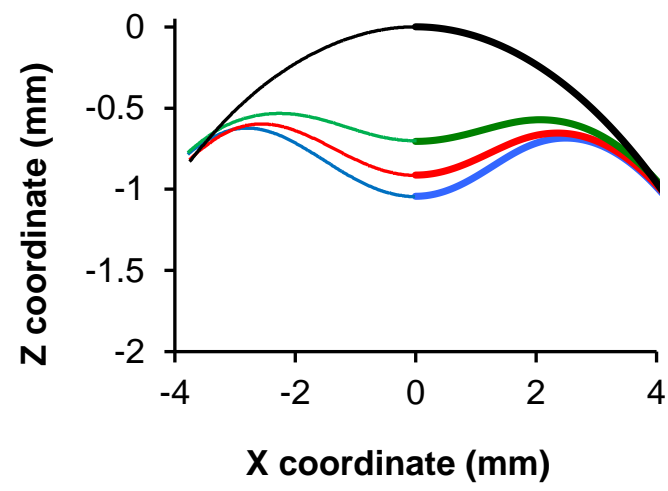

D)

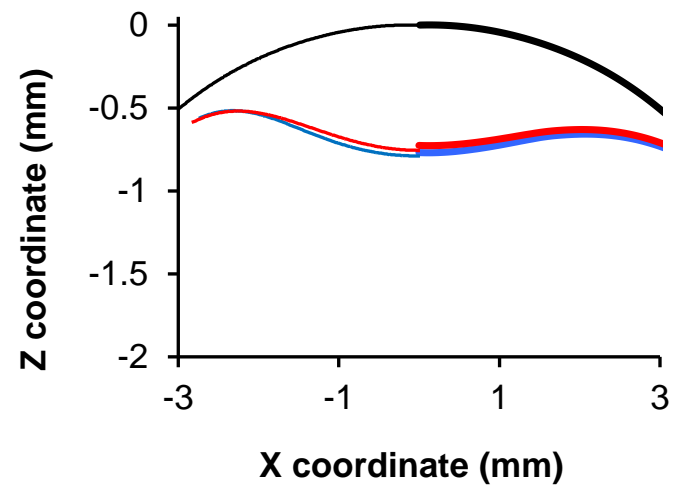

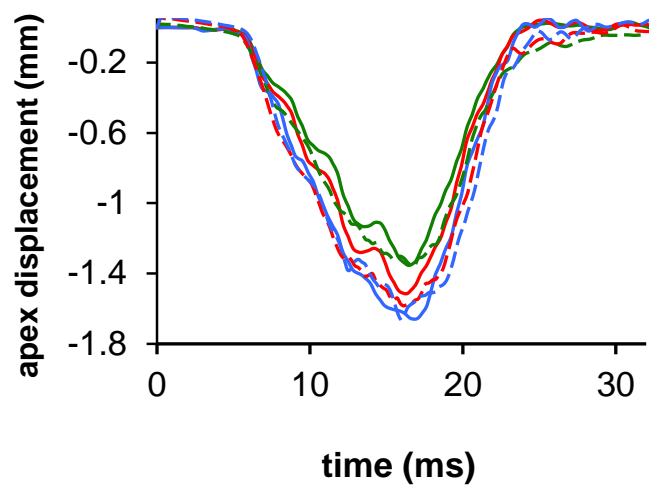
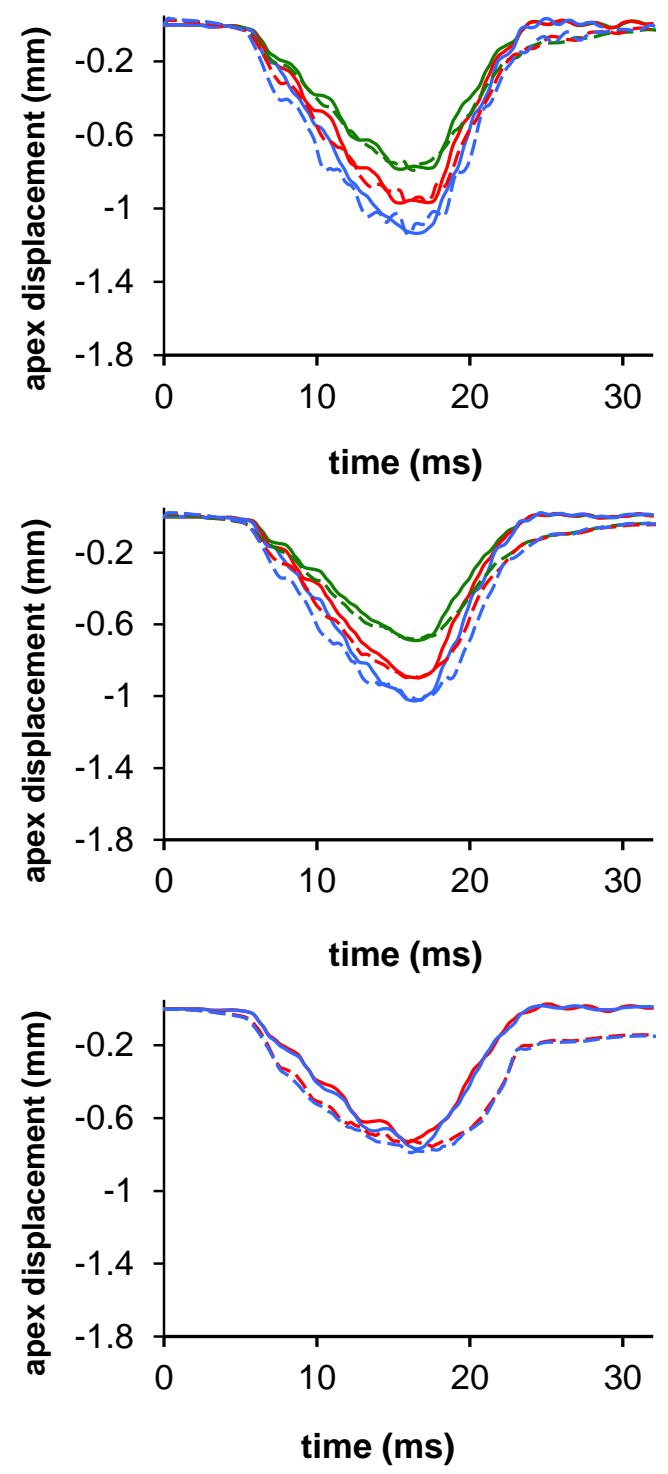

Corneal strips

\begin{tabular}{|llll|}
\hline$-1(\operatorname{sim})$ & $-2(\operatorname{sim})$ & $-3(\operatorname{sim})$ & - Initial $1(\operatorname{sim})$ \\
$---1(\exp )$ & $---2(\exp )$ & $---3(\exp )$ & --- Initial $1(\exp )$ \\
\hline
\end{tabular}

Figure 3.6: Spatial and temporal profiles of MC and PC deformation. For the deformed states, three trials (numbered as 1, 2, and 3) are represented. 


\subsection{Discussion}

The work presented in this chapter shows the validity and utility of an inverse modeling approach for retrieving biomechanical properties of the cornea through non-invasive means. High correspondence between simulated and experimental material and deformation parameters was found.

The method was validated using model polymer corneas with similar geometries and mechanical properties as real corneas. The method was found to accurately describe the mechanical properties of the cornea even accounting for differences in thickness. Air puff parameters were similar to those found in human corneas: $\mathrm{DA}=0.7-1.6 \mathrm{~mm}$ in this work compared to $0.9-1.26 \mathrm{~mm}$ in human corneas. In terms of material response, the main difference is that the stress-strain response of the MCs, while non-linear, can be described even using a single term (equivalent Young's Modulus). The corneas measured show larger non-linearity and a definition of Young's Modulus for them requires larger user interpretation and input. The cornea is comprised of collagen fibers, and collagen-based tissues have been experimentally shown to have non-linear, Jshaped material curves, arising from the progressive recruitment of strain-resistant components in certain loading conditions (Fratzl et al., 1998; Mitsuhashi et al., 2018).

Another important distinction is between temporal profiles. The constitutive models used in this work do not have a visco-elastic component and thus do not actively model time-dependent behavior. Polymer materials for contact lenses have a limited viscoelastic response, whereas the cornea is known to exhibit time-dependent behavior (Glass et al., 2008; Loewenstein \& Bettelheim, 1976; Zhang et al., 2017). For this reason, the simulated temporal profiles of MCs diverge less from their experimentally measured counterparts than the temporal profiles of porcine corneas.

A review of the literature indicates that the material properties retrieved from the inverse modeling procedure are in line with other techniques. Previous research has found corneal Young's modulus values of 0.6 to 3.0 MPa (see Section 1.2.1). The two porcine corneas measured showed different equivalent's Young Moduli (0.99 and 1.91 $\mathrm{MPa}$ ), both within the range of previous findings. The model developed in this work is two-dimensional and the material models used were isotropic, whereas the cornea is known to exhibit anisotropy (T-M Nguyen et al., 2014; Singh et al., 2016). 
Measurement of corneal biomechanics is particularly relevant to pathologies where spatial variations in stiffness are observed (keratoconus) and localized treatment (corneal cross-linking). Further validation work could be done to assess whether multiple material properties can be retrieved using the described protocol. This would likely require altering the merit function in order to incorporate additional data from the experimental measurements (such as deformation amplitude along specified nodes or coordinates). The main limitation of the approach is the two-dimensional nature of the measurements compared to the non-symmetrical nature of pathologies such as keratoconus. However, the use of displacement information from additional crosssectional images of the air puff imaging system could provide enough additional information to characterize a three-dimensional, non-symmetric response.

\subsection{Conclusions}

An inverse modeling, finite element procedure has been used to reconstruct material properties of model and porcine corneas using air pulse tonometry and Scheimpflug imaging. The procedure was able to accurately retrieve the material properties of corneas of different dimensions and reproduce stress-strain curves obtained through uniaxial extensiometry. The retrieved material properties and simulation accurately reproduced the spatial and temporal deformation profiles.

The work presented in this chapter provides a validation of reconstructing the mechanical properties of the cornea and contact lens polymer materials. This methodology has been applied to the evaluation of corneal treatments such as corneal collagen crosslinking with Rose Bengal and Green Light. In the next chapter, a novel biomaterial (silk-fibroin) in combination with Rose Bengal Green-Light is evaluated as a novel treatment for corneal wound healing. 


\section{Rose Bengal Photobonded Silk Fibroin Films for corneal wound healing}

\subsection{Introduction}

Penetrating wound injuries in the cornea are often treated by bandaging with a patch that protects the corneal surface and induces wound healing processes. The most common material used is amniotic membrane tissue, or amnion (Dua et al., 2004). Amnion is sutured onto the cornea, a time-consuming process that can also result in adverse reaction due to the injury caused by suturing.

A proposal to replace suturing is Rose Bengal Photobonding ( $\mathrm{RBPh})$, which consists of creating bonds between separate tissues by coating their surfaces with Rose Bengal, maintaining them in contact with each other, and irradiating with green light. $\mathrm{RBPh}$ has been proposed as a method for applications such as sealing lacerations(Tsao et al., 2012), or bonding corneal interfaces after LASIK surgery (Seiler et al., 2017). Researchers at the Wellman Center for Photomedicine found that amnion could be bonded to the cornea using Rose Bengal and green light, removing the need for sutures (Verter et al., 2011).

Amnion is frozen in a medium with glycerol or dimethylsulphoxide (DMSO) after it is obtained from the donor (after elective caesarian surgery). The preservation process has effects on the structural and chemical properties of the tissue, the most typical being the depletion of angiogenic factors (Gicquel et al., 2009; Wolbank et al., 2009). Various freezing methods have been proposed to solve some of these issues (Allen et al., 2013).

Amnion's origin as donor tissue and its preservation requirements are a limitation to its widespread usage. There is an interest in more readily available materials that can be used for the same application.

Bombyx mori silk fibroin is a biomaterial with widespread usage in biomedical engineering. It is readily accessible, biocompatible, can be used to form different types of structures (films, gels, foams), and its mechanical properties can be tailored to a desired application. Silk fibroin films have been explored as a material for corneal scaffolding, and have a number of advantages including mechanical strength and 
transparency. As such, silk is a candidate for use in corneal wound healing or grafting applications, and could have other possible uses in ophthalmology, should its engagement to ocular tissues be efficient.

The purpose of this work was to evaluate photochemical tissue bonding using silk fibroin (SF) films and corneal tissue.

\subsection{Protocols for silk film photobonding and evaluation}

Two types of bonding experiments were conducted in this work. The first was a uniaxial extension test, in which corneal strips and SF strips were photochemically bonded, and the force required to break the bond in uniaxial conditions was measured. These were the most extensive tests, done to evaluate and quantify the photobonding process. The second was an inflation test, in which a punctured ocular globe was covered with a SF disc dressing, and the surfaces were bonded. The maximum pressure required to break the bond was quantified.

\subsubsection{Silk film preparation}

Lyophilized silk fibroin was provided by the Biomaterials and Regenerative Medicine Engineering Laboratory at the Universidad Politécnica de Madrid.

A number of methods have been proposed to create insoluble silk films, such as water annealing, chemical treatment, and use of plasticizers such as glycerol(Lu et al., 2010). In this work, the latter method was used. This is a green chemistry approach and results in films that, unlike standard SF films, are flexible/ductile in their dry state, thus more suitable for manipulation. This method offers less control over the mechanical properties of the fibroin films than other proposed methods, but in the context of this experiment, fine-tuning mechanical properties was not a necessity.

The method is as follows. Solutions of silk fibroin and glycerol are mixed, such that the mass ratio of silk fibroin to glycerol is $75: 25$. This ratio is selected because previous studies have shown that higher than $20 \%$ glycerol is required to fully insolubilize the films after an immersion bath (Lu et al., 2010). The mixture is poured on a mold and allowed to set. After 36 hours, a silk fibroin-glycerol film is produced.

The fibroin-glycerol films were cut into strips with a width of $5 \mathrm{~mm}$ and a length of 15 $\mathrm{mm}$ (for the uniaxial extension test) or discs with an $8 \mathrm{~mm}$ diameter (for the inflation 
test). The strips or discs were then placed in distilled water for 3 hours, with the water changed every hour. The end result is a silk fibroin strip or disc that no longer has the ductility provided by the glycerol in its dry state.

\subsubsection{Rose Bengal preparation}

A $0.1 \%$ Rose Bengal solution was prepared by dissolving $0.01 \mathrm{~g}$ commercial RB sodium salt (Sigma-Aldrich Corp., St. Louis, MO, USA) into $10 \mathrm{~mL}$ of $\mathrm{H}_{2} 0$.

\subsubsection{Cornea preparation}

Porcine eyes were used for this experiment. All eyes were used no later than 48 hours after extraction.

\section{Uniaxial extension test}

For this experiment, the corneal epithelium was removed by applying alcohol to the corneal surface, then lightly scraping the epithelial layer with a scalpel. The cornea was then removed from the globe using surgical scissors, gently cutting along the interface between cornea and sclera.

The cutting device was used to cut a single strip from each cornea. The cutting device was applied to the center of the cornea in order to obtain a longer corneal strip.

\section{Inflation test}

For this experiment, the ocular globe was cleaned using surgical scissors to remove muscles and tissues, leaving only the globe. The optic nerve was cut and left at an extension of 3-4 $\mathrm{mm}$. The corneal epithelium was removed by applying alcohol to the corneal surface, then lightly wiping the epithelial layer with a scalpel.

The ocular globe was then mounted on a custom 3D-printed device. The device consists of a holder for the ocular globe, with a frontal cavity that exposes the cornea and a back cavity that exposes the rear of the globe and the optic nerve. The back of the holder has four rails, that allow for the insertion of a second piece, which restrains a $4 \mathrm{~cm}$ long needle. The ocular globe is first secured in the holder. Then, the second piece is moved forward, allowing it to pierce the optic nerve and remain stable.

After this, a puncture wound was created in the center of the cornea. The wound was applied on both axes, so that its shape resembled a cross. Each cut of the puncture had a 
length of approximately $2 \mathrm{~mm}$. The ocular globe was allowed to rest for a minute and any leaking fluid was dried with a paper towel.

\subsubsection{Photobonding}

\section{Uniaxial extension test}

Silk fibroin strips were fully immersed in the RB solution for 10 minutes. The strip was then removed from the solution, and its surfaces were dried by gently wiping it against a paper towel. The corneal strip's anterior surface was also dried with a paper towel. In addition, silk fibroin strips in other conditions were evaluated. Unstained silk fibroin films were used as controls for all irradiation conditions. Unstained silk fibroin films were also used as controls for non-irradiation condition (simple contact between surfaces). Finally, silk fibroin films stained in a $0.1 \%$ rhodamine solution were evaluated for one irradiation condition. Conditions are summarized in Table 4.1.

The corneal strip was placed on top of a container. Then, the silk fibroin strip was placed above the corneal strip, in such a way that $50 \%$ of the corneal strip was in contact with the silk fibroin strip. A metal washer with a central hole of $8 \mathrm{~mm}$ diameter was placed on top of the silk fibroin strip. This was done to maintain pressure and contact between the silk fibroin and corneal strips as well as to ensure consistency of the irradiated area.

The strips were placed under a solid-state green light laser source (CNI Tech, Co. Ltd., Changchun, China) and the light beam was centered on the washer. The strips were then irradiated with green light, at $0.175 \mathrm{~W} / \mathrm{cm}^{2}$ for different irradiation times $(0,5,10,15$ minutes). After the irradiation was completed, the washer was removed, and the two strips were carefully removed from the container. The process is represented in Figure 4.1.

\section{Inflation test}

SF discs were fully immersed in the RB solution for 10 minutes, then removed. Their surfaces were wiped of excess liquid. For each experiment, the SF disc was placed on the cornea of the ocular globe, aligned to the puncture wound.

The holder with the ocular globe was placed vertically, with the cornea facing the light beam. The metal washer was placed on top of the SF disc. The light beam was aligned 
with the washer, and the SF disc and ocular globe were irradiated with green light for a set amount of time. After irradiation was completed, the washer was removed, and the holder with the ocular globe was transferred to a pressure testing system. A diagram of the process can be seen in Figure 4.2.

\begin{tabular}{|ccc|}
\hline Condition & $\begin{array}{c}\text { Irradiation time at } \\
\mathbf{0 . 1 7 5 ~ W / \mathbf { c m } ^ { 2 }} \mathbf{( s )}\end{array}$ & $\begin{array}{c}\text { Number of } \\
\text { samples }\end{array}$ \\
RB-stained & 0 & 8 \\
RB-stained & 300 & 8 \\
RB-stained & 600 & 8 \\
RB-stained & 900 & 8 \\
Rhodamine-stained & 600 & 8 \\
Unstained & 0 & 8 \\
Unstained & 300 & 8 \\
Unstained & 600 & 8 \\
Unstained & 900 & 8 \\
\hline
\end{tabular}

Table 4.1: Summary of experimental conditions for SF strips.

A)

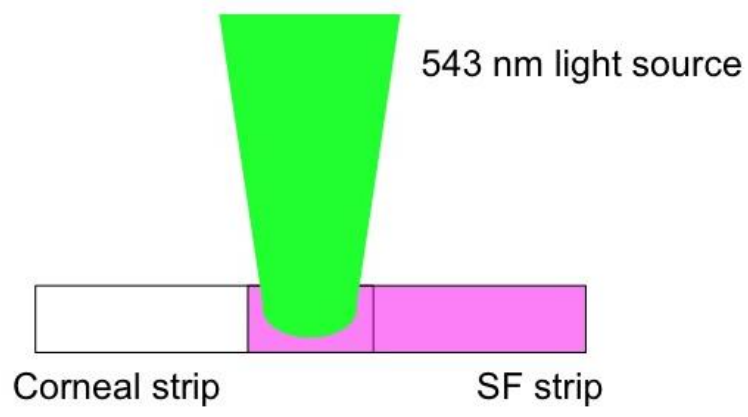

B)

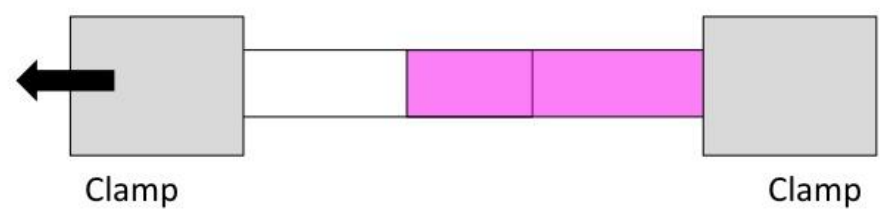

Uniaxial stretcher

Figure 4.1: SF strip protocol. A) Strips of SF (stained or unstained) are placed above a corneal strip and irradiated with green light. B) Bonded strips are placed on uniaxial stretcher and the maximum force before the bond breaks is recorded. 
A)

B)
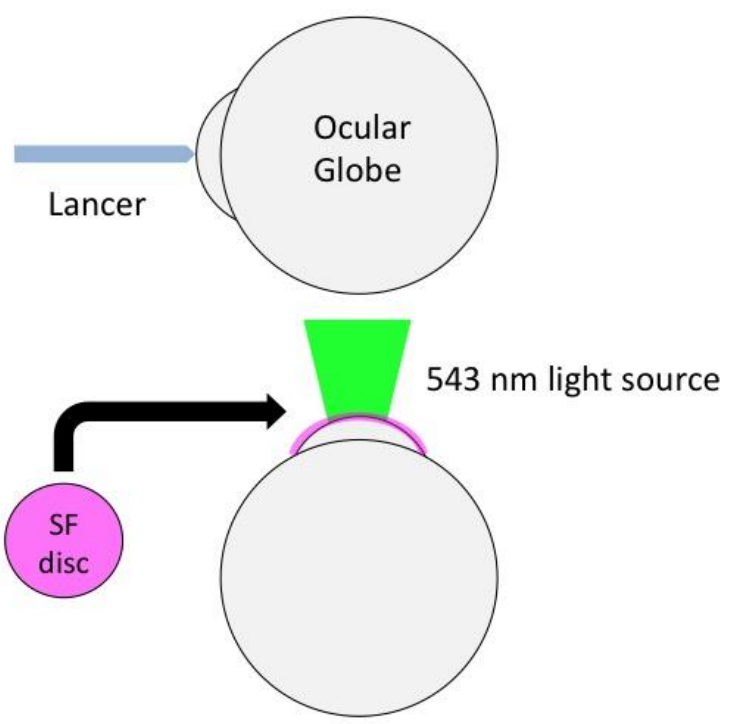

C)

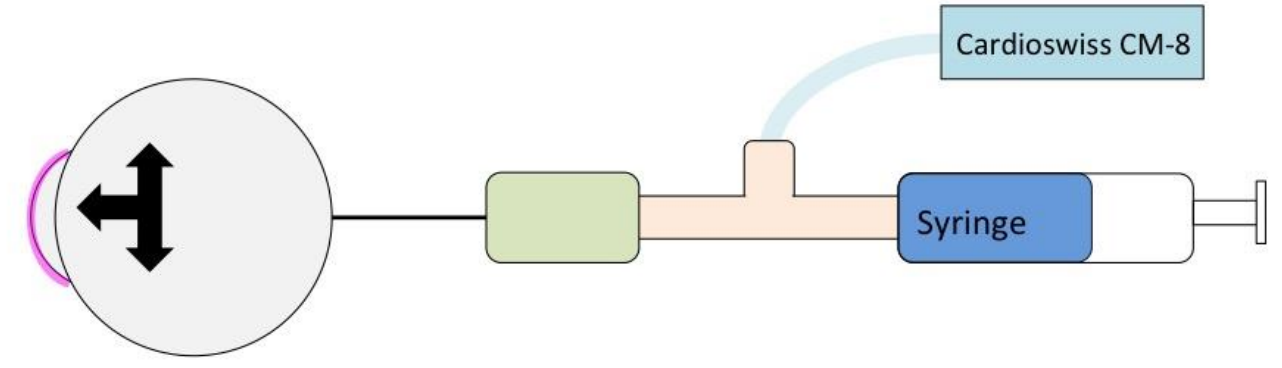

Figure 4.2: Inflation testing protocol. A) Cornea is penetrated with a lancer to simulate a wound. B) RBstained SF disc is placed above cornea and both are irradiated with green light. C) Syringe is used to fill ocular globe with water. Maximum pressure (from connection to EKG) is recorded.

\subsubsection{Mechanical Testing}

\section{Uniaxial extension test}

The CellScale UStretch uniaxial testing system (described in Section 2.1.3) was used to apply a uniaxial tensile force to the SF-cornea strip in order to measure the bonding strength.

The SF-cornea strips were mounted and fastened on the system's mechanical clamps. The SF strip was mounted on the right clamp, and the cornea strip on the left clamp. The experiments were conducted in immersion. This choice was made because previous testing found that dryness (from the irradiation and from general exposure to air) could result in false positives, as there is some natural adhesion between silk and the corneal surface. Ensuring that the SF strip and cornea were properly hydrated eliminated this effect. In order to test in immersion conditions, a water chamber was filled with tap 
water at room temperature. The SF and corneal strips were allowed to hydrate for 10 minutes.

A single uniaxial measurement was conducted for each sample. The measurement consists of extending the corneal strip at a rate of $1 \mathrm{~mm} / \mathrm{s}$ until failure of the bond or the samples. A photo capture of the uniaxial testing can be seen in Figure 4.3.

The data was then processed using a custom MATLAB code. The code zeroed the force values and extracted the maximum force before breakage, calculated the standard deviation with measurements in the same conditions, and compared the data to measurements done under different conditions.

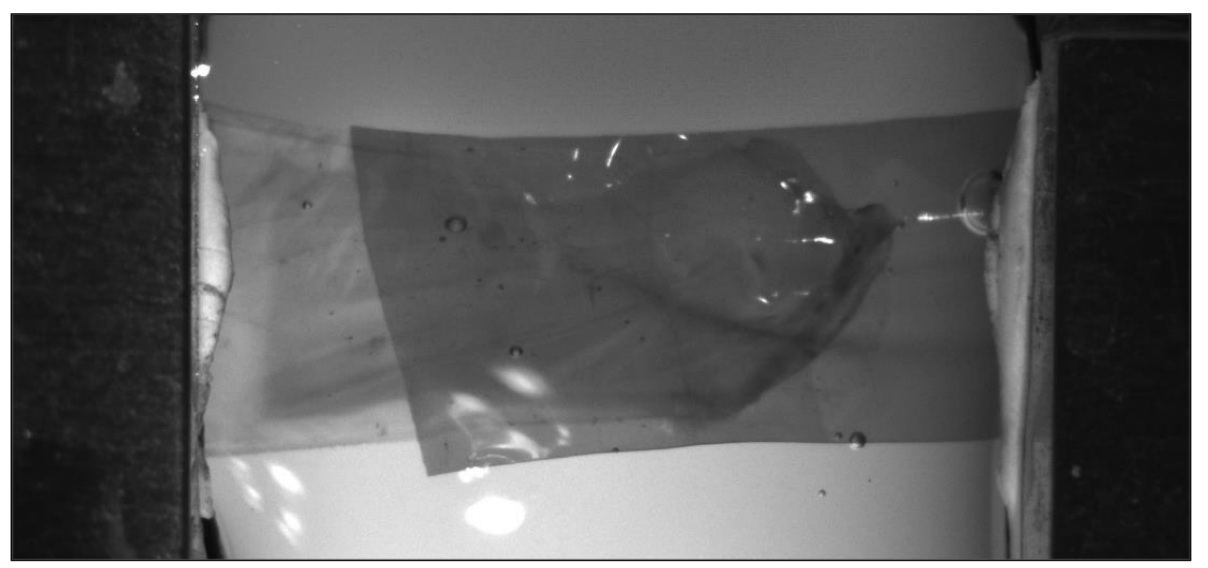

Figure 4.3: SF strip and corneal strip pulled apart in uniaxial stretcher.

\section{Inflation test}

A Cardioswiss CM-8 device (Schiller $\mathrm{Ag}$, Switzerland) was used to measure the pressure during inflation. This is a patient monitoring device used to record pressure. A three-way valve was used, with one opening having a $10 \mu \mathrm{m}$ syringe filled with water attached to it, the central opening connecting to the CM-8, and the final opening being attached to the hollow needle that was previously inserted into the ocular globe.

The syringe was used to inflate the ocular globe, and the pressure was read by the system. The syringe was manually operated and the device's screen was recorded, in order to obtain the pressure. The ocular globe was inflated until bursting was observed. The maximum pressure was recorded. 


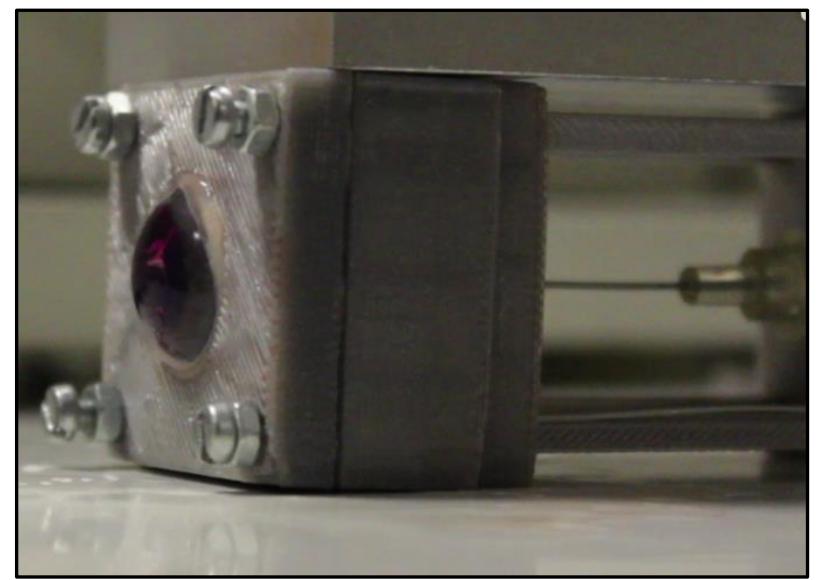

Figure 4.4: Inflation testing. Ocular globe is held inside container; syringe enters the lens through the optic nerve and applies water pressure.

\subsection{Mechanical performance of Rose Bengal photobonding}

\subsubsection{Uniaxial extension test}

Figure 4.5 shows the results of all RB-stained strips and control strips for all conditions. Each experimental condition is represented by its median value. The results show that, for RB-stained strips, there is a nearly linear increase in the maximum force obtained experimentally as a function of fluence (energy per unit area). The longer the strip is irradiated, the higher the required force to break the bond. For fluence up to $105 \mathrm{~J} / \mathrm{cm}^{2}$, the maximum force is recorded when the bond between the SF strip and the corneal strip is broken. However, for the highest fluence value $\left(157 \mathrm{~J} / \mathrm{cm}^{2}\right)$, the SF strips would break before the bond itself. This happened in 6 of the 8 measured samples. This is a function of the mechanical and physical properties of the SF strip. Given this outcome, and the magnitude of the fluence values, it was considered unnecessary to continue measuring for higher fluence.

The control strips show a different trend. The results show a small increase in the force required to break the bond when some irradiation is done on an unstained strip. However, additional irradiation does not seem to have a meaningful effect on the maximum force recorded. This suggests that there is some adhesion effect caused by irradiating both surfaces in contact which each other. The deviation in samples increased for the largest fluence value.

An additional control condition was evaluated but is not represented graphically. These were SF strips that were neither stained nor irradiated, but simply allowed to remain in 
contact with a corneal strip for the same periods of time as other strips were irradiated. These samples were mounted on the stretcher, but whatever bond the two strips had to each other did not remain during immersion, preventing any extension measurements. This is further evidence that there is at least a baseline level of adhesion from irradiation and contact, which is nonetheless lower than the bond generated through Rose Bengal and irradiation.
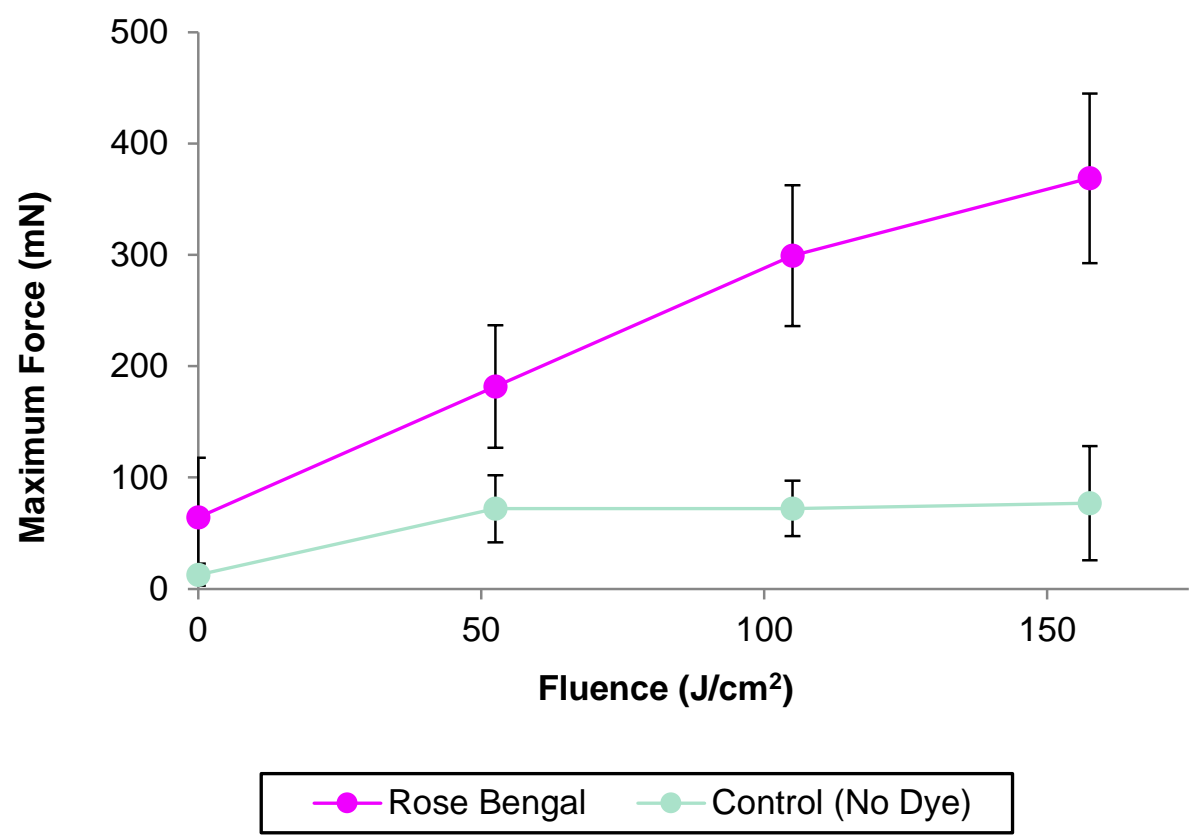

Figure 4.5: Maximum force from extensiometry testing as a function of fluence

Figure 4.6 shows an additional control: a comparison between SF strips stained with Rose Bengal, SF strips stained with rhodamine, and control (unstained) strips, for a fluence of $105 \mathrm{~J} / \mathrm{cm}^{2}$. The results show that, while the maximum forces in SF strips stained with Rose Bengal are higher than those found with control strips, the rhodamine-stained strips had the same maximum forces as the control strips. This suggests that Rose Bengal and light are generating a real photochemical bonding process, rather than the bonding being a result of increased heat adhesion due to light absorbance. 


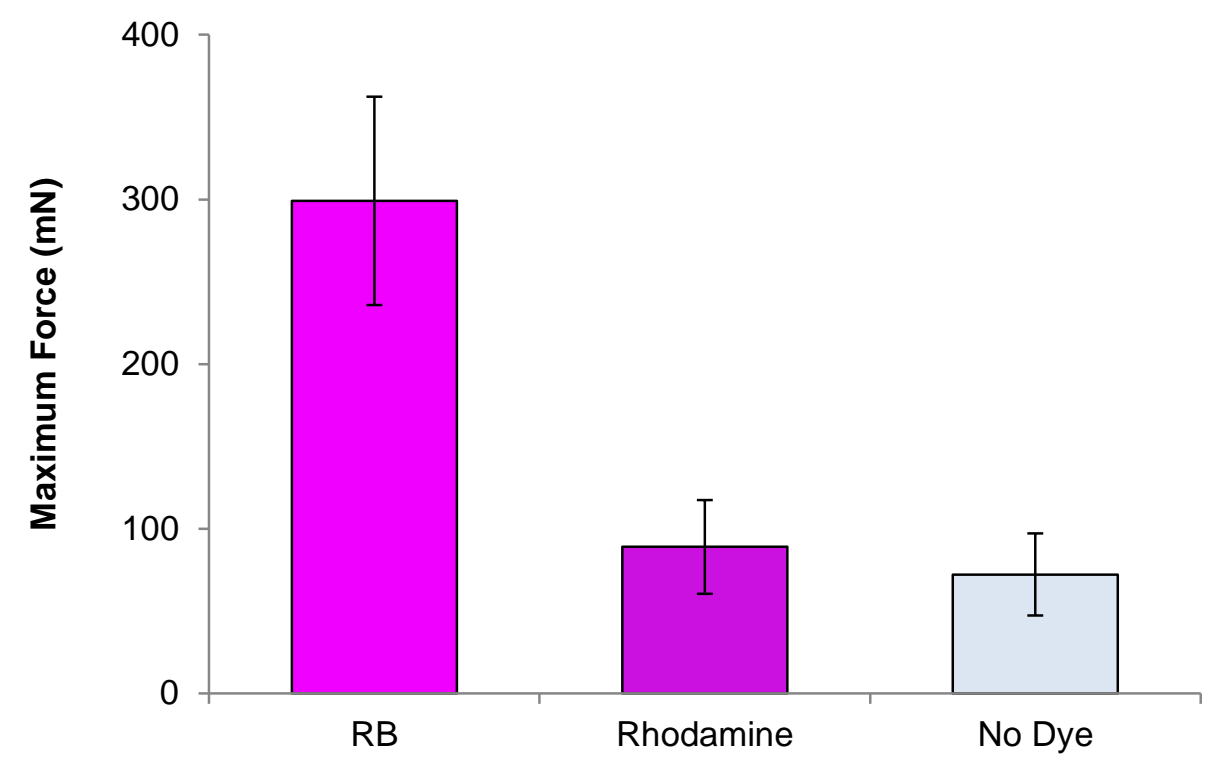

Figure 4.6: Comparison between RB-stained, rhodamine-stained, and unstained SF strips. Both controls result in significantly lower maximum force values than the RB-stained strips. Fluence in all samples was $105 \mathrm{~J} / \mathrm{cm}^{2}$.

\subsubsection{Inflation testing}

Figure 4.7 shows the results obtained from inflation testing. The influence of irradiation time on the maximum pressure values can also be observed.

The maximum pressure was the result of two situations. The first was when the SF disc would burst, typically from a puncture in its surface, close to where the corneal puncture wound was located. The second was when leakage would begin at a corner of the SF disc. As the SF discs are a flat surface, bonded against a spherical surface like the cornea, the disc could not always hermetically seal around the surface. After testing, the SF discs were manually removed from the cornea by peeling. The discs appeared to remain bonded to the cornea regardless of the bursting. The pressure recorded is beyond the intraocular pressure the eye typically experiences and resulted in substantial deformation of the ocular globe.

\subsubsection{RB effect on silk properties}

Figure 4.8 shows the results of evaluating for maximum stress and strain (in terms of stretch ratio) for RB-stained and control films. The maximum stretch before break was consistent for both conditions. However, the maximum stress before break was found to be lower for RB-stained, irradiated silk films. Further analysis found that the overall stiffness in the stained films was only $72 \%$ of the overall stiffness in the unstained 
films. Qualitative, it was observed that RB-stained, irradiated silk films in their dry state appeared more brittle than control films.

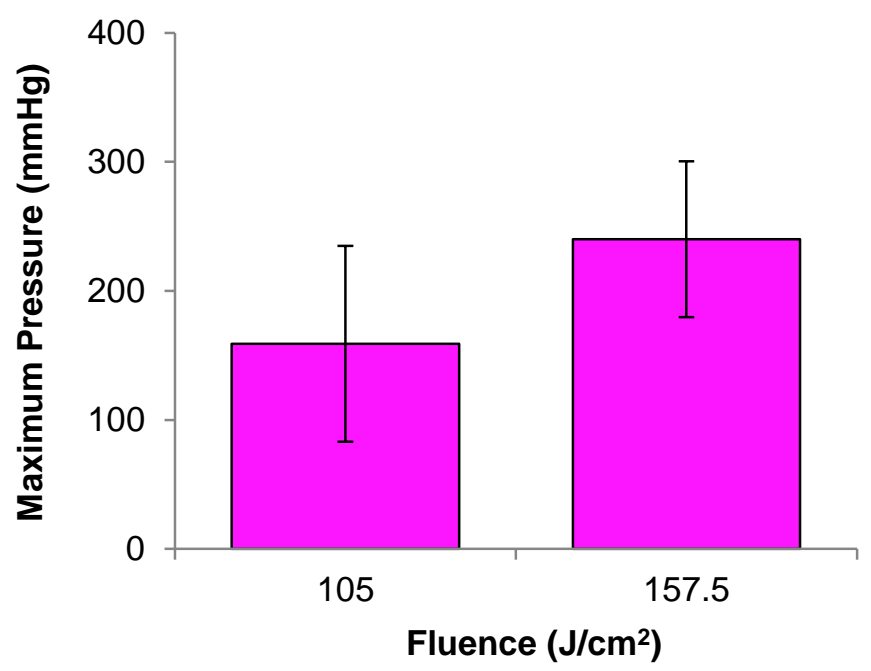

Figure 4.7: Maximum pressure recorded for RB-stained SF discs bonded onto cornea. The effect of increasing fluence is reflected in the data.

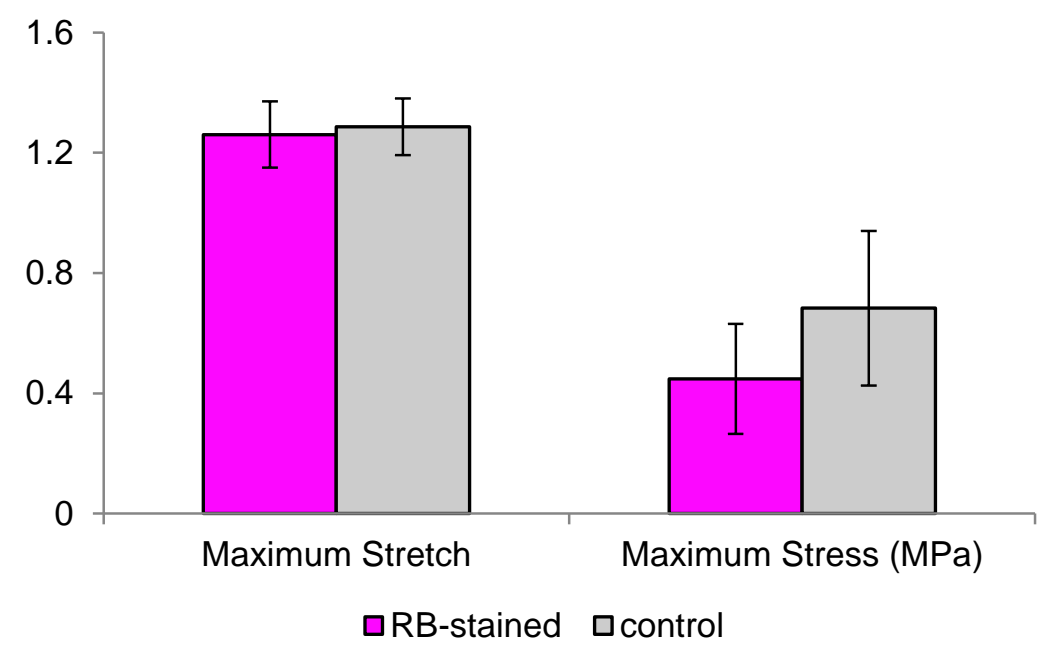

Figure 4.8: Maximum stretch and stress values found in RB-stained and control films. Although the average maximum stretch before breaking was consistent among both conditions, the recorded force and stress for RB-stained films was lower. Fluence: $105 \mathrm{~J} / \mathrm{cm}^{2}$.

\subsection{Discussion}

The results from these experiments show that Rose Bengal photobonding can be used to bond silk fibroin, in its film form, to the corneal tissue. The strength of the bond can be mediated by the irradiation dose. The photobonding appears to be distinct from other 
adhesion phenomena between silk and cornea, which were reduced as much as possible during these experiments.

The variation in the data is somewhat high, especially for RB-stained, bonded strips/discs. The analysis of the data is focused on maximum force values. Although the SF strips were all made in the same mold, some variations in thickness are expected and these could have an influence on the maximum force values. For the strip extension testing, an analysis of the data showed that, when bonding appears to occur (at maximum force values higher than $100 \mathrm{mN}$ ), it is more likely for there to be outliers in the data, especially on the higher end of the maximum force readings. Photobonding depends on uniform, dry contact between surfaces, and although efforts were made to guarantee uniformity in bonding surface area across all experiments, it's possible that some strips had a better bonding environment than others, resulting in stronger bonding. It is also possible that surface irregularities contribute to bonding strength. For these reasons, the results were presented in terms of the median value.

An important finding is that photobonding appears to have an effect on the mechanical properties of the SF films. This is not surprising, as Rose Bengal is already a subject of study for corneal crosslinking. In this case, the SF films appear to be weaker mechanically, not stronger, after irradiation. This has important implications in the use of Rose Bengal to bond silk to the cornea. For applications in which the specific mechanical properties of the film are very important, it could be necessary to limit the use of Rose Bengal to peripheral areas (where the bond is most necessary) or to alter the pre-irradiation mechanical properties to account for the expected post-irradiation change.

Amnion is metabolically active and its growth factors are believed to be responsible for its utility as a corneal bandage or graft. The viability of using silk fibroin films for this application has to be studied further, in cell culture and animal models, in order to assess healing and growth response. Although evidence exists that silk films are effective for cell growth (T-1 Liu et al., 2010), further studies would need to evaluate wound healing and post-implantation transparency. Growth factors can be added to silk films using various techniques (Zhou et al., 2016), and the topography of silk films can also be altered to encourage corneal cell growth in specific directions, improving transparency (Lawrence et al., 2009). Silk films also exhibit poor permeability, which a 
long-term application would need to improve using a method such as treating the films with PEG (Suzuki et al., 2015). Additionally, mechanical properties and overall thickness will have to be tailored to the application. A final formulation of SF films for corneal healing would likely require additional design input.

\subsection{Conclusions}

This chapter shows that it is possible to photochemically bond silk fibroin, in film form, to corneal surfaces, and that the strength of the bond can be modulated by the irradiation time. The force magnitude and speed of the process makes this an application with potential interest for researchers in corneal reconstruction and wound healing.

The last two chapters focused on material properties of the cornea and a new approach (using photochemical bonding) for corneal treatment. Similar frameworks for material reconstruction of material properties can be used for the other ocular component in the eye (the crystalline lens), and explore new approaches for a correction of the most prevalent condition in the lens (presbyopia), as will be presented in the next two chapters. 



\section{Material properties of the crystalline lens from Brillouin microscopy and numerical simulations}

\subsection{Introduction}

Brillouin optical microscopy is a novel technique that can be used to study a material's viscoelastic properties in a non-invasive and speedy way, and can quantify spatial differences in elasticity within a sample at a high resolution. Brillouin microscopy has potential applications in ophthalmology, allowing for non-invasive study of the mechanical properties of the two most relevant tissues of the anterior segment: the cornea and the crystalline lens (see Section 1.4.2).

The crystalline lens has an opto-mechanical function: its mechanical deformation produces a change in optical power. It is generally understood that its two main regions, the nucleus and cortex, have distinct mechanical properties. Additionally, increased lens stiffness is believed to be associated with presbyopia, the loss of accommodation capability. Brillouin optical microscopy is suitable to quantify these differences.

A number of studies on crystalline lens analysis using Brillouin optical microscopy have been published (Bailey et al., 2010; Besner et al., 2016; Reiß et al., 2011; Scarcelli et al., 2011). The most distinctive finding in these papers is that higher Longitudinal Modulus values (the standard output of Brillouin optical microscopy) are found in the nucleus, and lower values are found in the cortex, for lenses of all ages. Recent findings have shown little age-related variation in minimum and maximum longitudinal moduli values within the lens (Besner et al., 2016). These findings are at odds with those obtained from other techniques, which typically find lower Young's or shear modulus in the nucleus than in the cortex, at least in younger eyes (Wilde et al., 2012).

This discrepancy calls into question whether the longitudinal modulus values obtained from Brillouin microscopy are providing accurate or useful information about the crystalline lens' mechanical properties and behavior. Direct correlation of Brillouin microscopy and other mechanical data is complicated; the contents of the lens are very soft and difficult to evaluate with traditional material characterization techniques. Scarcelli et al evaluated the shear and longitudinal modulus of a small number of lens 
Material properties of the crystalline lens from Brillouin microscopy and numerical simulations

fragments and found a logarithmic relationship between both moduli (Scarcelli et al., 2011).

The goal of this work is to evaluate whether data obtained from Brillouin microscopy can be used to accurately reconstruct the mechanical behavior of the crystalline. Two finite element models of the crystalline lens were built. The first is a model of a lens undergoing compression. This model uses experimental data from lens compression and Brillouin microscopy as its inputs, and an inverse modeling approach is used to generate an elasticity map for the lens that is consistent with both data. The second model consists of a crystalline lens undergoing accommodation. The output from the first model (material properties) is used as an input for the second model, in order to observe the effects of a spatially varying elasticity map on the changes in thickness, surface curvature, and power of the crystalline lens.

\subsection{Numerical analysis of the crystalline lens properties based on Brillouin microscopy measurements}

\subsubsection{Measurements}

The University of Maryland provided the experimental data used in this work.

The datasets consisted of compression and Brillouin microscopy measurements of two human crystalline lenses, of 14 and 37 years of age. The lenses were de-capsulated before measurements. Each lens was measured using a custom-built Brillouin optical microscope. The technical specifications of this device are described in detail in the researchers' previous published work (Scarcelli et al., 2011).

The Brillouin microscopy system (described in Section 2.1.5) was used to obtain a 2dimensional cross section of the lens. The spatial resolution of the Brillouin microscopy measurements was of $0.2 \mathrm{~mm}$ along the $\mathrm{x}$ and $\mathrm{y}$-axes. The output of the measurements was a spatial map of Brillouin frequency shifts. The frequency shift is related to the Longitudinal modulus by the following equation:

$$
M^{\prime}=\frac{\rho \Omega^{2} \lambda^{2}}{4 n^{2} \sin ^{2}\left(\frac{\theta}{2}\right)}
$$


Where $\Omega$ is the Brillouin frequency shift, $\lambda$ is the optical wavelength of the system (532 $\mathrm{nm}), \rho$ is the mass density, $\mathrm{n}$ is the refractive index, and $\theta$ is the angle of measurement. The angle of measurement was $180^{\circ}$. The mass density and refractive index vary spatially across the lens. In the researchers' previous work, the ratio of $\rho / n^{2}$ was calculated and found to be constant, with an approximate value of $0.564 \mathrm{~g} / \mathrm{cm}^{3}$. These values were used to create a spatial map of longitudinal moduli values for each lens. Figure 5.1A shows a representative Brillouin map.

Following the Brillouin microscopy measurements, each lens was compressed using a custom-built compression device. The lenses were compressed by an 8mm-diameter piston. The force response and the displacement/position of the piston were the outputs of the system. Each lens was compressed once, and a maximum displacement of 1.5 mm was applied to the piston. The displacement-force data was analyzed and fitted to a 5-term polynomial equation. Figure 5.1B shows the compression setup.
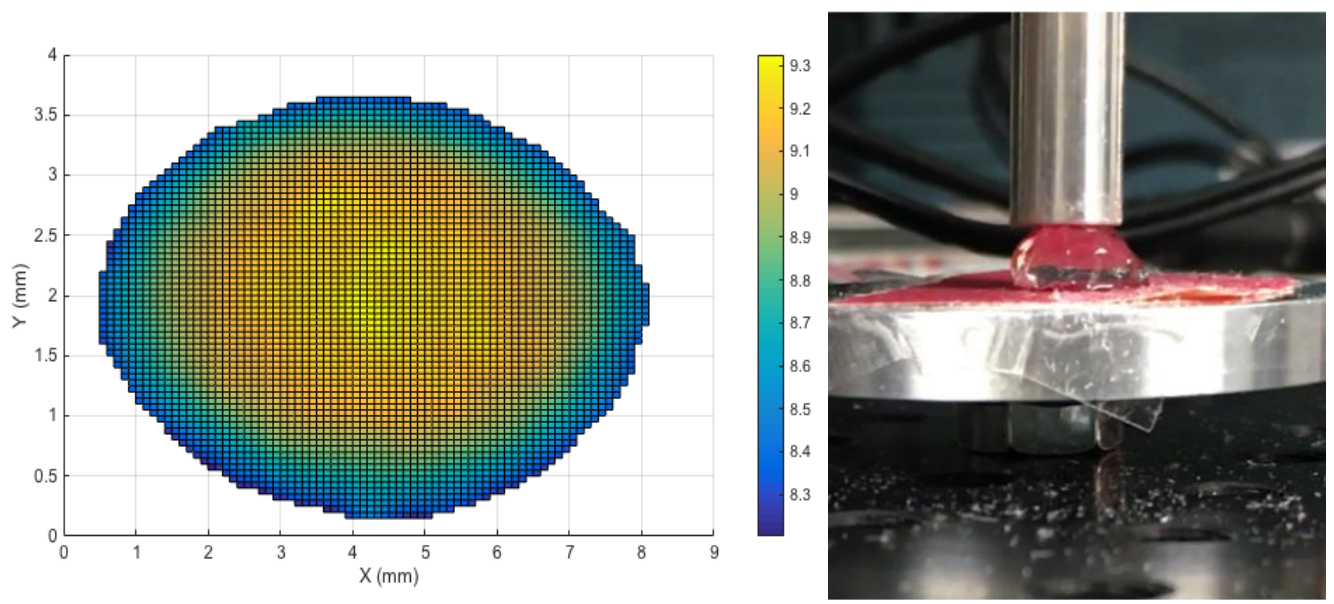

Figure 5.1: A) Brillouin frequency shift map of the same lens (GHz). B) Compression of human crystalline lens.

\subsubsection{Finite Element Model of Lens Compression}

\section{Lens Geometry}

The coordinate values from the Brillouin elasticity map were used to reconstruct the geometry of the crystalline lenses. The y-values were smoothed and fitted with splines in order to obtain a realistic representation of a smooth lens surface. The importance of this lies on the fact that the compression test, in this case, is a contact problem between a rigid and deformable surface. The force-displacement relationship depends on the area of contact between the compression piston and the lens. For a displacement of $1 \mathrm{~mm}$, 
Material properties of the crystalline lens from Brillouin microscopy and numerical simulations

the area of compression was $50 \%$ of the total lens diameter for the 14-year-old lens (smallest in overall size).

\section{Elements \& Mesh}

The crystalline lenses were reconstructed using ANSYS Mechanical APDL (described in Section 2.2.1). The crystalline lens was modeled as a 2-dimensional, axisymmetric model. Only the left half of the lens geometry was modeled. The lenses were meshed using PLANE183 elements. Figure 5.2 shows the meshed model. The surface on which the lens rested was modeled as a $0.5 \times 6 \mathrm{~mm}$ cylinder and the compression piston was modeled as a $0.5 \times 4 \mathrm{~mm}$ cylinder. Both structures were assigned PLANE183 elements. Two contact regions were modeled: the first, between the anterior lens surface and the compression piston, and the second between the posterior lens surface and the bottom surface/floor. The contact was modeled as frictionless, flexible surface-to-surface contact, using TARGE169 and CONTA172 elements.

An analysis found that the effect of element size on the final output of the model was minor $(<5 \%)$. As such, it was possible to use an element edge length of $0.2 \mathrm{~mm}$, which reduced computation time and matches the resolution of the Brillouin elasticity map.

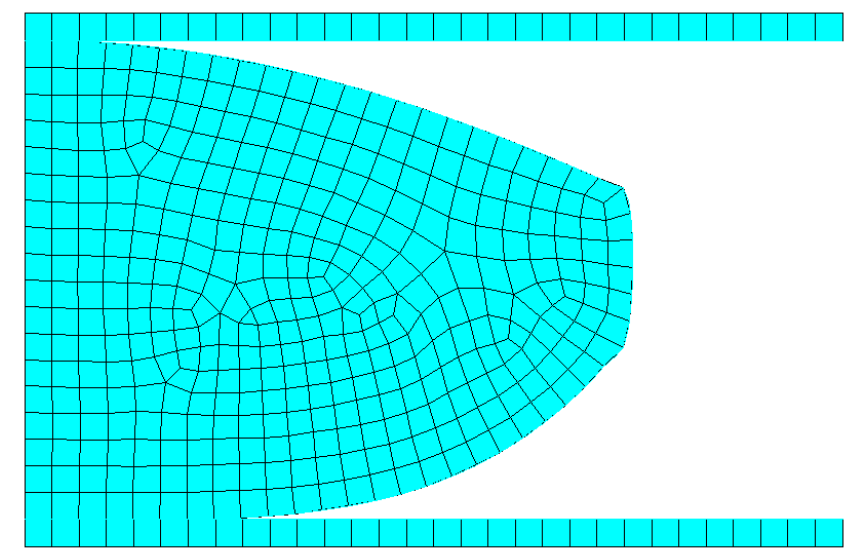

Figure 5.2: Finite element model of lens compression

\section{Material Properties}

ANSYS Mechanical APDL does not provide material models that account for spatially varying properties. In order to implement this feature into the model, an APDL code was developed. 
The code imports the Brillouin elasticity maps to the APDL environment. The $\mathrm{x}$ and $\mathrm{y}$ coordinates and longitudinal moduli are stored as arrays within the finite element model. The longitudinal moduli values are then converted to Young's modulus, using the empirical relationship found by Scarcelli et al:

$$
\operatorname{alog}(E)+b=\log B
$$

Where $a$ and $b$ are constants determined experimentally, $B$ is the longitudinal modulus, and $E$ is the Young's modulus.

The elements within the crystalline lens are initially assigned a linear elastic material model with a Young's modulus value of $1 \mathrm{MPa}$ and a poisson's ratio of 0.49 . The code retrieves the centroid of each element, and finds the nearest coordinates in the arrays of the Brillouin elasticity map. This value is used to replace the initial assigned material.

The Neo-Hookean coefficient is equivalent to the material shear modulus and is linearly related to the Young's modulus (1.39). The constitutive model used in this simulation was a Neo-Hookean model (described in Section 1.5.2), and the calculated Young's modulus was converted to shear modulus before being assigned to each element. The final gradient of elasticity is discrete rather than continuous.

\section{Solution}

Compression was modeled by displacing the compression piston $1 \mathrm{~mm}$ downward along the $y$-axis. The inferior surface was fixed along the $\mathrm{x}$ - and $\mathrm{y}$-axes. Analysis was set at "Large Displacement - Static" and the solution was broken into 50 substeps. The data generated for all substeps was stored and the finite element model was solved.

\section{Post-Processing}

After solving, the force reaction was computed from the addition of the component forces at the compression piston. The displacement was computed from the position of the uppermost node of the crystalline lens. Displacement and force reaction were computed for all 50 substeps.

The sum of the squared error between the simulated and the experimental forcedisplacement data was calculated. The experimental data was represented by the 5 -term polynomial equation fits. This sum was the output of the finite element model. 
Material properties of the crystalline lens from Brillouin microscopy and numerical simulations

$$
\Phi=\sum\left(f_{m}-f_{\text {sim }}\right)^{2}
$$

\section{Inverse Modeling}

The finite element models of the crystalline lens were used to develop an inverse modeling process. The general structure of this type of model is described in Section 2.2.2.

The ANSYS Mechanical APDL optimization environment was used for this process. The finite element code for the crystalline lens was assigned as the analysis file. Two design variables were assigned: $a$ and $b$, the parameters used to convert the longitudinal modulus to Young's modulus. A pre-analysis was done to calculate Young's moduli for various combinations of $a$ and $b$ parameters and evaluate the ratio of maximum and minimum moduli within the lens. Parameters resulting in ratios higher than 1000 were discarded, both for plausibility (outside the range of most experimental work on crystalline lens stiffness) and model stability (solution was unlikely to converge with very high differences in stiffness within the lens). With this pre-analysis, boundaries of $a=0.01-0.5$ and $b=7.5-9.4$ were assigned to the variables. The sum of the squared errors between experimental and simulated force-displacement was selected as the objective function to minimize. A first-order analysis was selected, with a maximum iteration of 50 and a percent step size of 10 .

After minimizing the sum of the squared errors and retrieving a material model that fitted the compression data, the simulated force-displacement was compared to the experimental values.

Initially, the objective was to obtain a single elasticity map (in terms of Young's modulus) for each of the two lenses evaluated. However, it was found that the optimization process could not find a global minimum, and the parameter combinations were linearly related. The force-displacement relationship of the lens under compression is defined by its overall stiffness, and cannot be described by two unique values. Due to this, a series of elasticity maps whose force-displacement relationship matched the experimental relationship were obtained and used for the next models. 


\subsubsection{Finite Element Model of Accommodation}

The accommodation model used in this work is based on a model developed by Burd and collaborators (Burd et al., 2002). Their model uses experimental data from Fisher in order to model the accommodation of the crystalline lens for different ages. The model and its underlying experimental data have been replicated or reused extensively in accommodation models (Lanchares et al., 2012; K Wang et al., 2016), as there is a paucity of mechanical and geometric data to build finite element models of accommodation.

Burd et al modeled lenses of three different ages: 11, 29, and 45. The 11-year-old model was deemed by Burd to yield inaccurate or plausible accommodation data. For the purposes of this work, only the 29 and 45 -year model data were used.

\section{Lens Geometry}

The geometric parameters of the two lens models, as well as the mechanical properties, are summarized in Table 5.1.

\section{Elements \& Mesh}

The crystalline lens was meshed using PLANE183 axisymmetric elements. Elements were refined along the external surface of the lens and assigned an element edge length of $0.05 \mathrm{~mm}$. The crystalline lens capsule was modeled using SHELL208 axisymmetric membrane elements and assigned a constant thickness of $0.013 \mathrm{~mm}$. This is a simplification from Burd's model, in which a more sophisticated implementation of spatially varying capsular thickness was implemented.

\begin{tabular}{|ccc|}
\hline Dimension & $\begin{array}{c}\text { 29-year-old } \\
\text { model }\end{array}$ & $\begin{array}{c}\text { 45-year-old } \\
\text { model }\end{array}$ \\
Equatorial Diameter & 4.3136 & 4.448 \\
Ciliary Body Diameter & 6.474 & 6.33 \\
Lens Thickness & 4.13 & 4.84 \\
Anterior Radius of Curvature & 6.5 & 7.6 \\
Posterior Radius of Curvature & 5.3 & 8.1 \\
Nucleus Equatorial Offset & 0.5119 & 0.6292 \\
\hline
\end{tabular}

Table 5.1: Geometry data for crystalline lenses of 29 and 45 years, from Burd 2002.

The lens' zonules were modeled as springs and assigned COMBIN14 elements. 
Material properties of the crystalline lens from Brillouin microscopy and numerical simulations

\section{Material Properties}

Mapping the Brillouin elasticity maps directly onto the lens accommodation models was not viable due to the differences in geometry. Instead, the spatially varying material models were reproduced using a gradient elasticity model. The elasticity map is defined as:

$$
E(r, \theta)=E_{n}-\Delta E\left(\frac{r}{r_{s}}\right)^{k}
$$

In this equation, $\mathrm{E}_{\mathrm{n}}$ is the Young's Modulus of the central region of the lens, $\Delta E$ is $\mathrm{E}_{\mathrm{n}}$ $E_{s}$, where $E_{s}$ is the Young's Modulus at the periphery of the lens, $r$ is the distance between center and centroid of the element at $\theta, \mathrm{r}_{\mathrm{s}}$ is the distance between the center and surface at $\theta$, and $k$ is the gradient coefficient, which defines the form of the transition between central and peripheral moduli.

A material assignment procedure similar to the one used for the lens compression model was implemented in the accommodation model. The code calculated the centroid of each element, computed the corresponding Young's modulus, converted the value to shear modulus, and assigned this value to the element. $\mathrm{K}$ was calculated from the Brillouin elasticity maps (at $y=0$ and $x=0$ ) and found to be 5 for the 14 -year old and 7 for the 37-year-old lens maps.

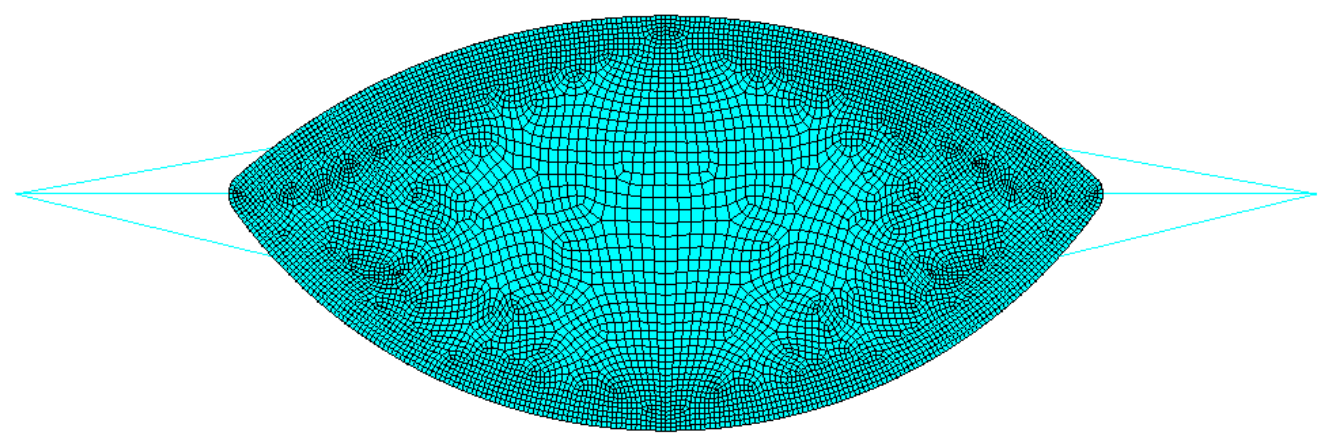

Figure 5.3: Finite element model of accommodation for 29-year-old lens

The elasticity models from the lenses aged 14 and 37 were used in the 29-year-old and 45-year-old accommodation models. 
The material models from Burd's model were used as controls. The nucleus and cortex values for lenses aged 29 and 45 were used as the $E_{n}$ and $E_{s}$ values in the gradient elasticity model.

The values for capsule elasticity were calculated in the same manner as the Burd model, in which experimental data (Krag et al., 1997) is used to obtain the following relationship:

$$
E_{\text {cap }}=a(A-35)+1.45
$$

Where $A$ is the age (in years) and $a=0.03 \mathrm{~N} / \mathrm{mm}^{2}$ for $A<35$ and $a=0$ for $A>35$.

The material properties of the zonules were assigned as spring constants. The procedure to select the spring constant value was adapted from the Burd model. A spring constant value was found such that the ciliary body displacement and lens radius diameter in the disaccommodated state matched experimental displacement data. The full model is represented in Figure 5.3.

\section{Solution}

Two different boundary conditions were evaluated. The first was a force of $0.08 \mathrm{~N}$ applied to the ciliary body. The second was a displacement applied to the ciliary body. The magnitude of the displacement was adapted from the data used in Burd's model, and was defined as:

$$
\delta(m m)=0.5129-0.00525 * A
$$

Where $A$ is age in years.

The analysis type was set at "Large Displacement - Static" and assigned a substep count of 50. Only the data from the last substep was stored.

The experimental data used by Burd et al did not have geometric or physical parameters as outputs, which is its main limitation. It is not possible to compare the output of the finite element model to specific experimental data on surface curvature and thickness. Instead, the power of the lens in its accommodated (undeformed) and disaccommodated (deformed) states can be calculated using surface curvature and thickness data. The 
accommodation amplitude can be compared to expected amplitude in that age range to assess the credibility and plausibility of the model.

To quantify the surfaces in their undeformed and deformed states, a code was implemented in ANSYS. The code adapts an open source MATLAB program called Circle Fit (Pratt Method), which fits a circle to a set of data points using a method described in the literature. This method was chosen because it is non-iterative; the ANSYS APDL environment does not readily allow the introduction of iterative operations as part of pre- and post-processing. The initial and final position of the nodes along the anterior and posterior surfaces of the crystalline lens were used as input. Only the nodes along a central radius of $1 \mathrm{~mm}$ were used.

The thickness in the deformed state was calculated from the final positions of the upperand lowermost nodes. With the surface radii and thickness, the lens power was calculated using the thick lens equation:

$$
\text { Optical power }=\frac{n_{1}-n_{a}}{r_{a}}+\frac{n_{1}-n_{a}}{r_{p}}-\frac{t\left(n_{1}-n_{a}\right)^{2}}{r_{a} r_{p} n_{1}}
$$

Where $n_{1}$ is the refractive index of the lens (a value of 1.42 is used), and $n_{a}$ is the refractive index of the aqueous and vitreous (1.336).

The difference between optical power in deformed and undeformed states is computed as the accommodative amplitude.

\subsection{Crystalline lens properties and accommodation amplitude}

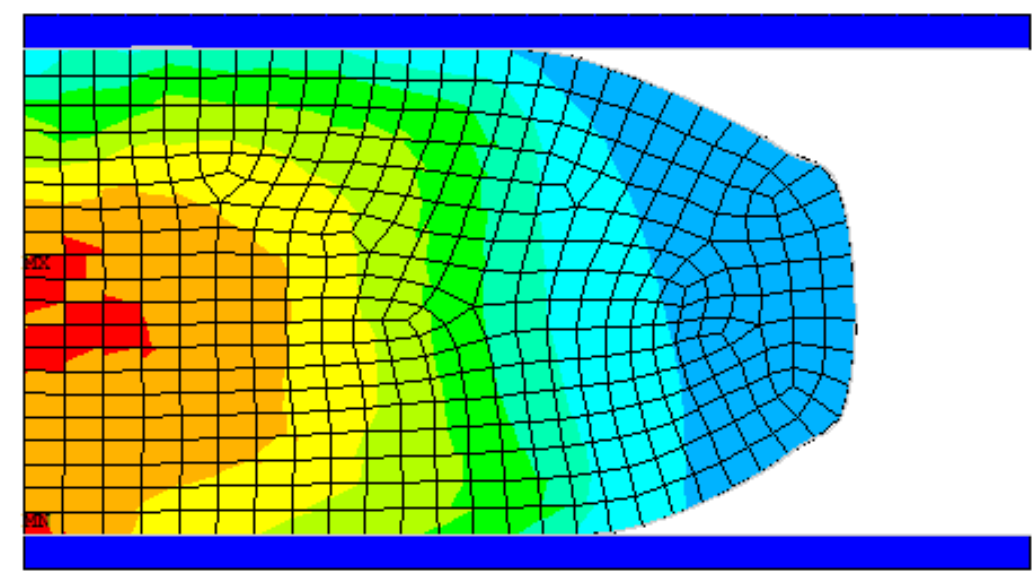

Figure 5.4: Lens compression model after solving. Von Mises strain. 


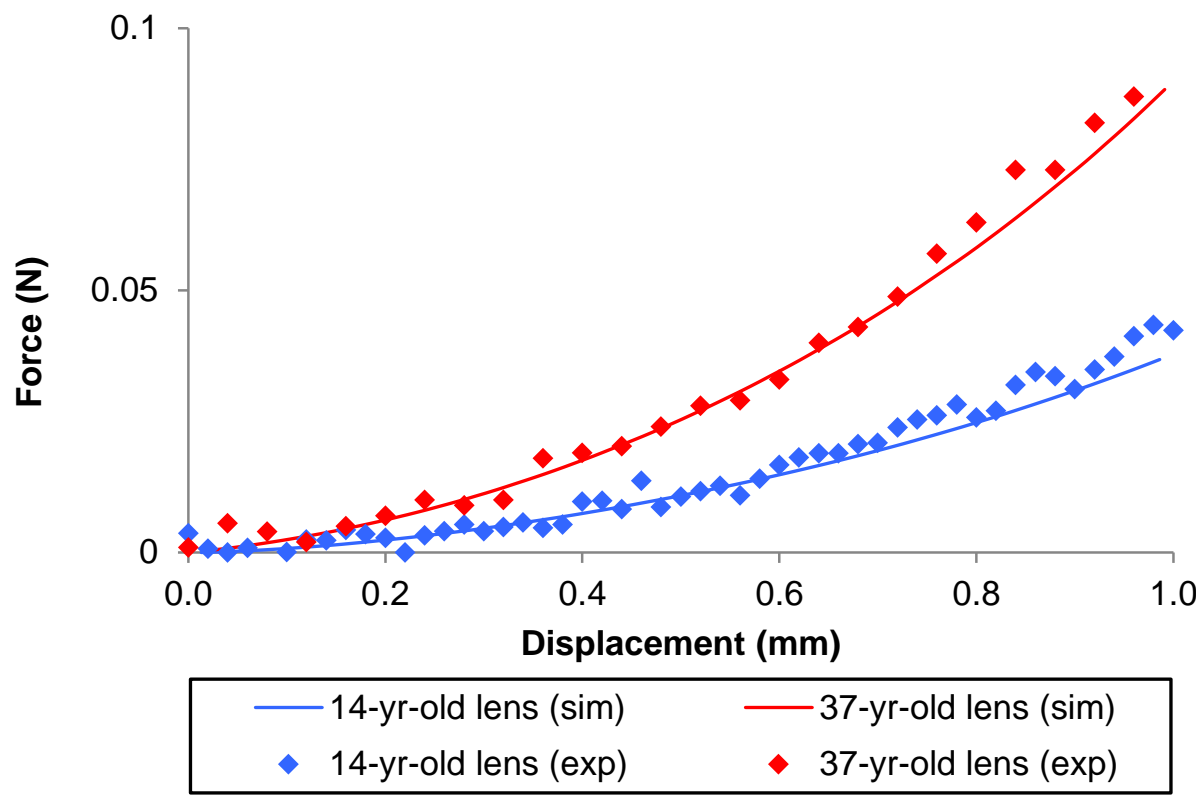

Figure 5.5: Simulated and experimental compression results from 14 and 37 year old lenses.

Figure 5.5 shows the results of the compression testing on both 14- and 37-year-old lenses. The simulated results represent the output of the model after minimization of the merit function.

\subsubsection{Accommodation models}

Figure 5.6 shows the accommodative amplitude obtained using three different Brillouin elasticity maps retrieved from the 14-year-old lens, compared to the amplitude obtained using data from Burd/Fisher, in a 29-year-old lens model. The boundary condition was a displacement of $0.29 \mathrm{~mm}$ on the ciliary body. There is a considerable difference between the amplitude obtained using the Brillouin elasticity maps and the Burd/Fisher data. The Burd/Fisher model has an accommodation amplitude of 5.9 D whereas the Brillouin models yield an amplitude of 2.5 D. The 14-year-old lens would be expected to show larger range of accommodation amplitude.

Figure 5.7 shows the accommodative amplitude for the models retrieved from the 37 year-old lens. The results are similar as in the 14-year-old lens. This is partly due to the displacement boundary condition. By imposing a radial deformation of a certain magnitude, the influence of the overall stiffness is reduced.

Figure 5.8 shows the accommodative amplitude for the 14-year-old elasticity models using a force boundary. $1 \mathrm{D}$ of amplitude is lost when using this boundary condition, on 
account of the overall stiffness for the Brillouin elasticity models being higher than the stiffness of the Burd model.

Figure 5.9 shows a reconstruction of the anterior and posterior surfaces of the lens using a Brillouin elasticity model and the Burd model. The reconstruction shows that the Burd model, where $E_{s}>E_{n}$, shows larger central deformation, decreased thickness, and flatter surfaces. The Brillouin model can be seen to deform less in the central area and slightly more in the peripheral region closer to the zonules.

The evaluated models used $k$ values of 5 to 7 , as determined from a previous evaluation of the Brillouin elasticity maps. The effect of the $\mathrm{k}$ value on the results was evaluated and the results are presented in Figure 5.10, for the first Brillouin elasticity maps of ages 14 and 37, compared to Burd's data for a 29-year-old lens. The results give insight into the effect of this elasticity distribution on the accommodative amplitude. The elasticity map is more homogenous when $\mathrm{k}$ increases, with higher $\mathrm{k}$ values representing an almost entirely homogenous lens. The results show that, for the Burd model, an increase in the value of $k$ reduces the accommodative amplitude. For the Brillouin models, the opposite is true: an increase in the value of $k$ increases the accommodative amplitude. The ratio of nuclear to cortical or peripheral stiffness has an important role in determining the accommodative amplitude. As $k$ increases, the majority of the lens' volume is described by $\mathrm{E}_{\mathrm{n}}$, and in practice becomes a homogenous solid.

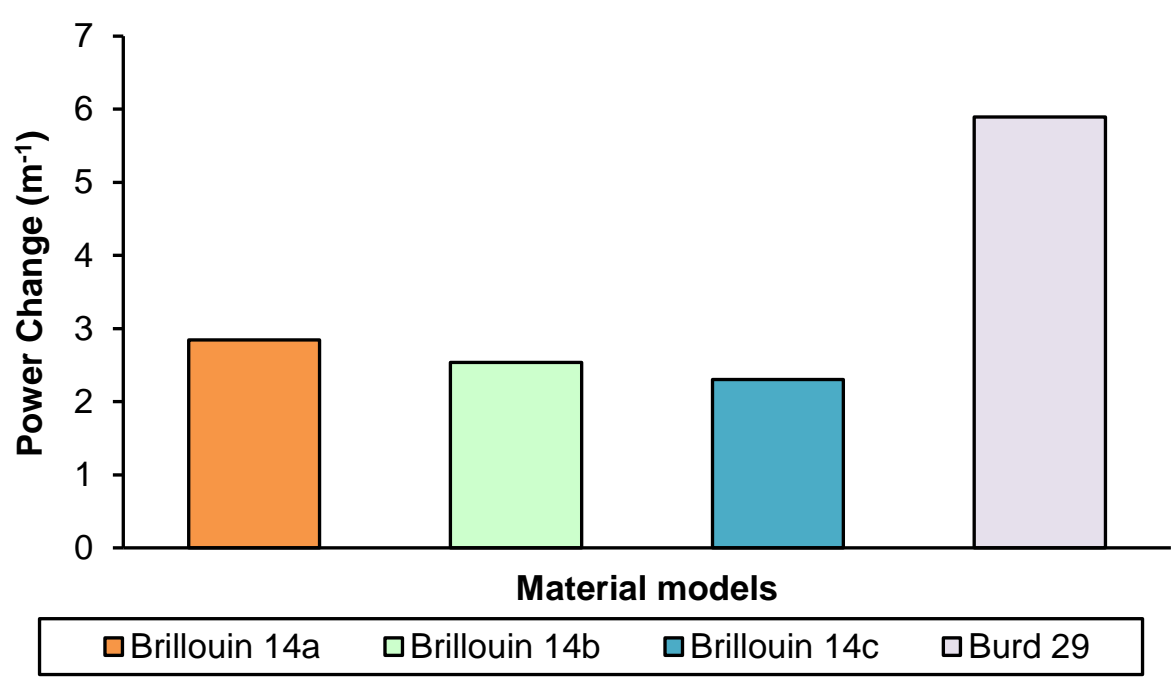

Figure 5.6: Accommodative amplitude of 14-year-old elasticity maps and 29-year-old data from Burd. Boundary condition: displacement. 


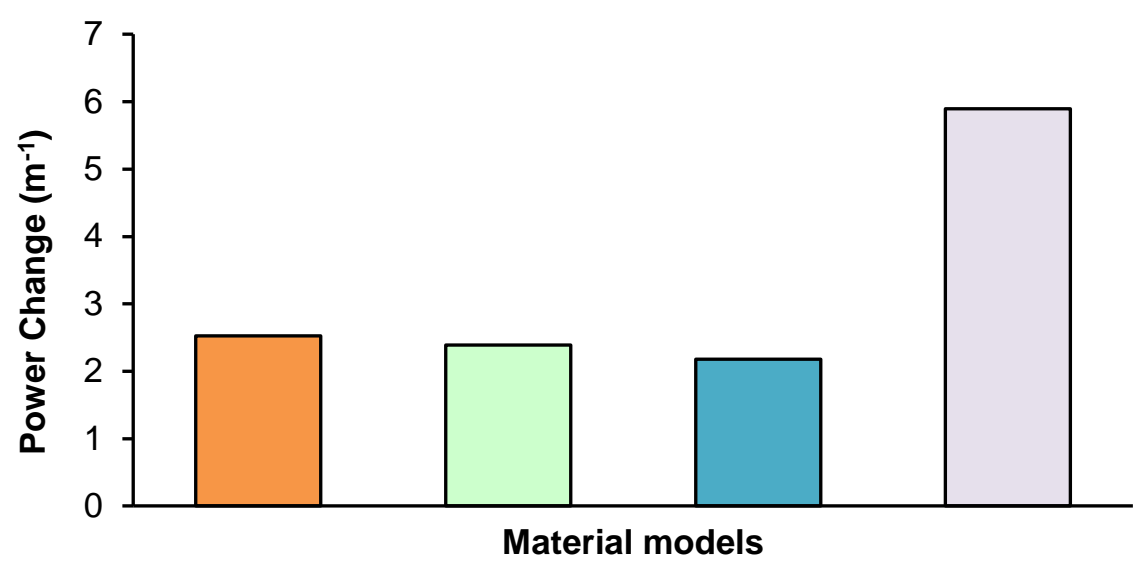

$\square$ Brillouin 37c $\quad$ BBillouin 37a $\quad$ BBillouin 37b $\quad$ aBurd 29

Figure 5.7: Accommodative amplitude of 37-year-old elasticity maps and 29-year-old data from Burd. Boundary condition: displacement.

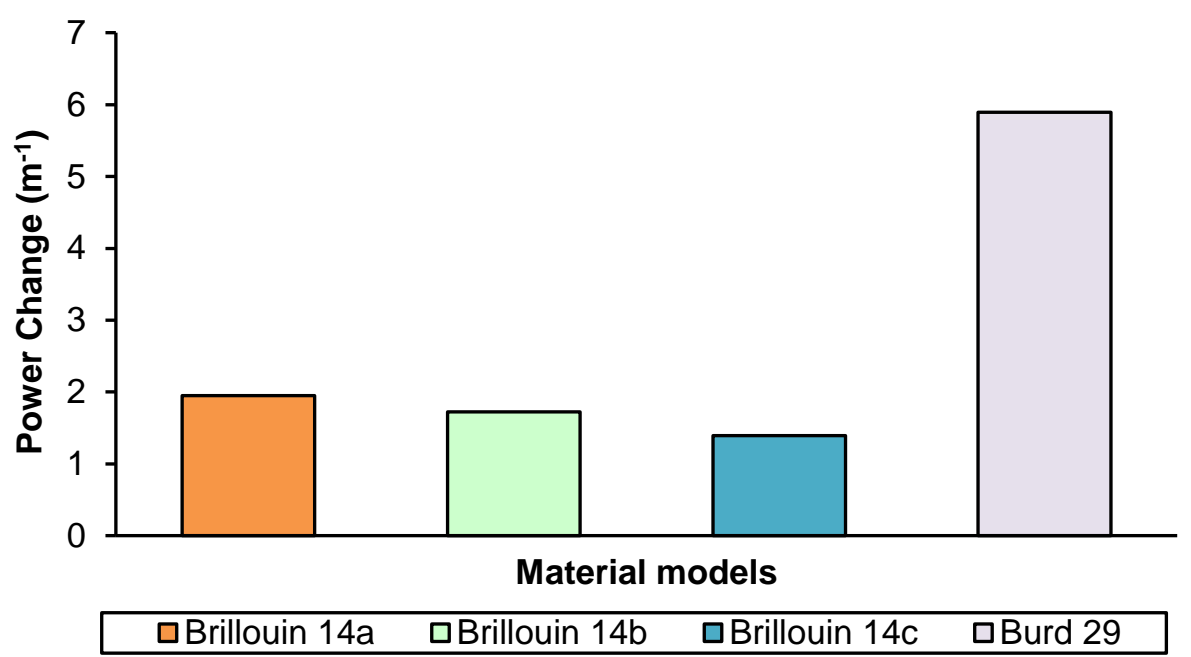

Figure 5.8: Accommodative amplitude of 14-year-old elasticity maps and 29-year-old data from Burd. Boundary condition: $0.08 \mathrm{~N}$ force. 
Material properties of the crystalline lens from Brillouin microscopy and numerical simulations

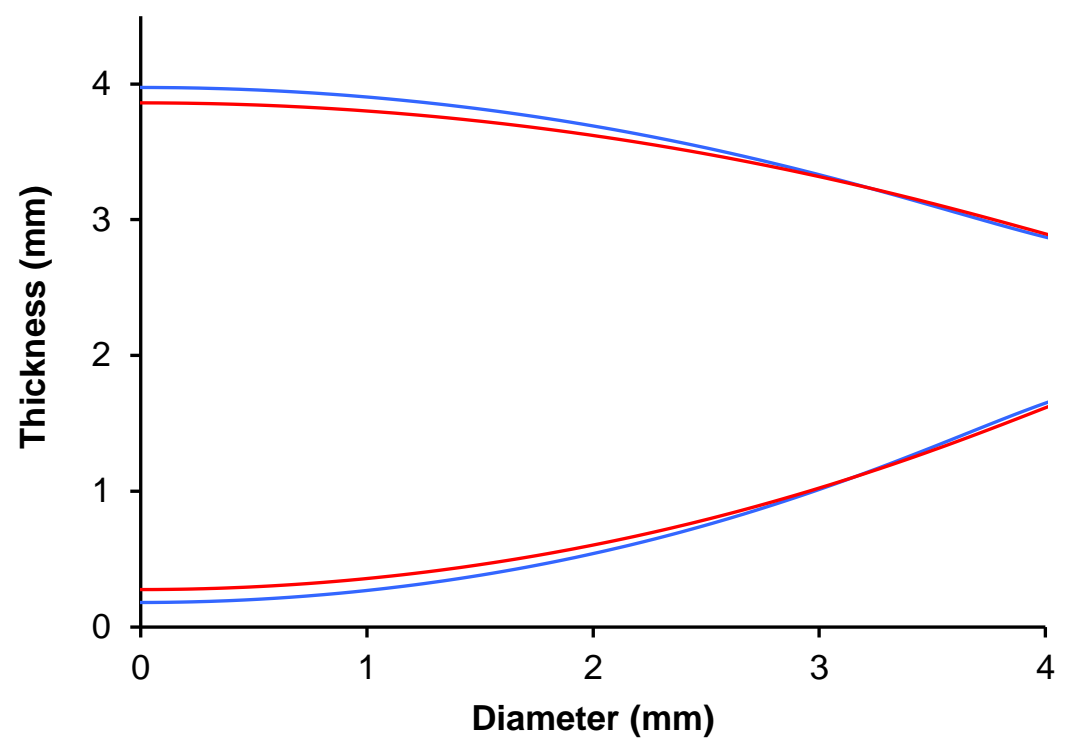

Brillouin Model —_Burd Model

Figure 5.9: Comparison between deformed surfaces of Brillouin and Burd material properties in accommodation models.

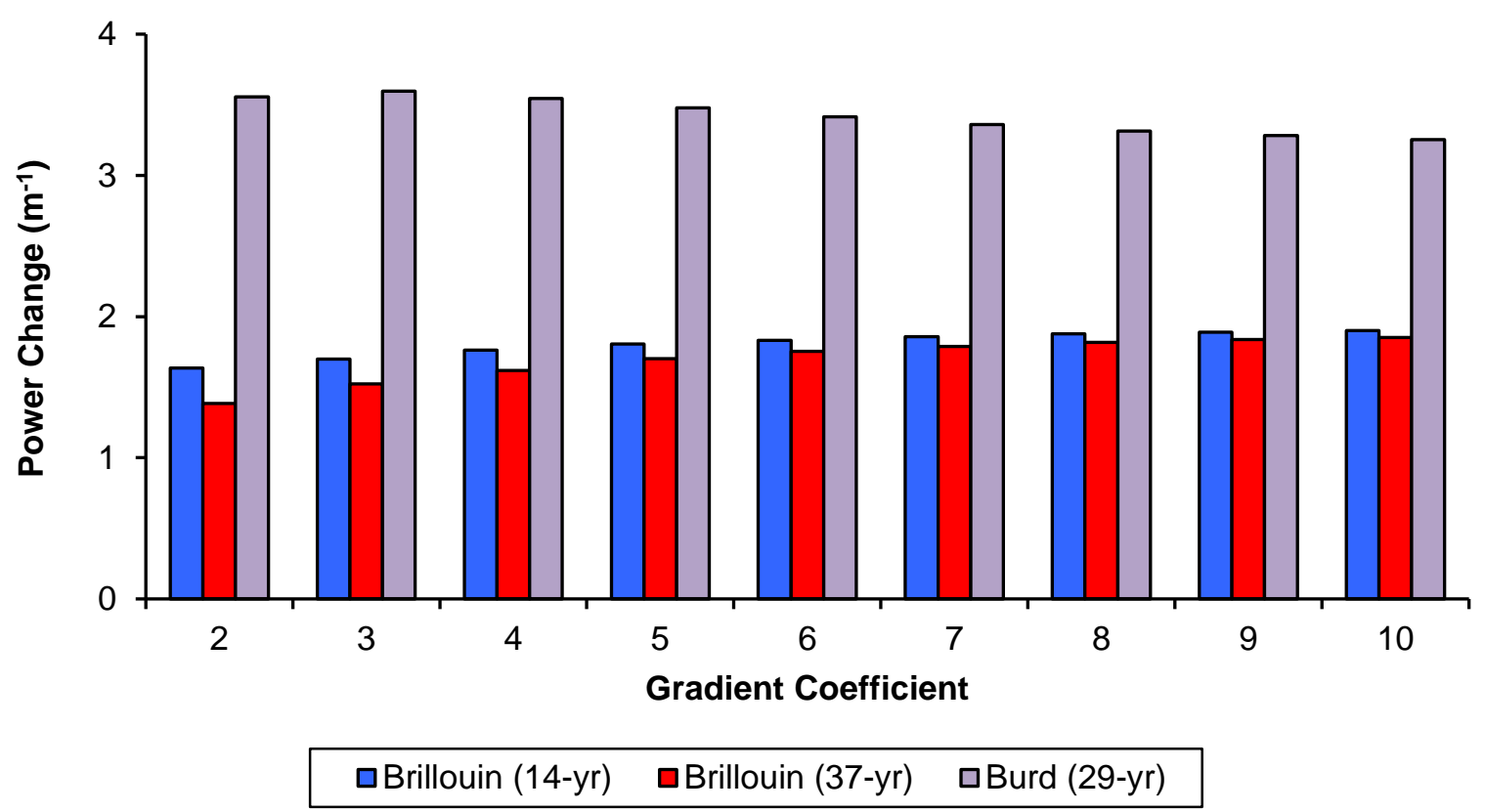

Figure 5.10: Evaluation of elasticity models with varying gradient coefficient $k$.

The Brillouin elasticity models obtained from the experimental data exhibit overall stiffness that is higher than the one calculated from the Burd models. This accounts for some of the difference in accommodative amplitude, although applying the 
displacement load should reduce this dependency on stiffness magnitude. To verify that these differences in magnitude where not the cause of the findings, new elasticity models were generated that were a fraction of the previously evaluated models.

Figure 5.11 shows the result of evaluating these models. The accommodative amplitude is seen to increase when the Brillouin elasticity map is a smaller fraction of the original. In order to obtain accommodative amplitude similar to the one obtained by the Burd material model, an elasticity map with a magnitude of only $5 \%$ of the original one is required. This is considerably lower stiffness than both the Burd model and the original Brillouin model. Although experimental conditions might affect the measured stiffness, there is no reason to expect such a large discrepancy. This further suggests that a crystalline lens with a stiffer nucleus than cortex or periphery is less suited for accommodation than one with a stiffer cortex.

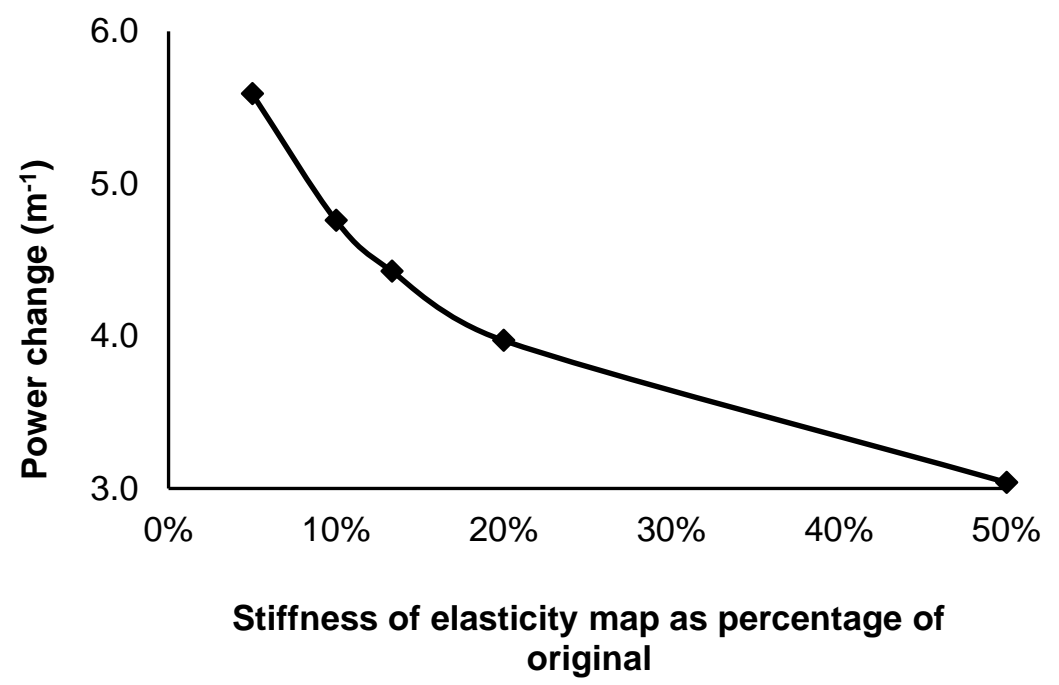

Figure 5.11: Change in accommodative amplitude for reduced elasticity maps.

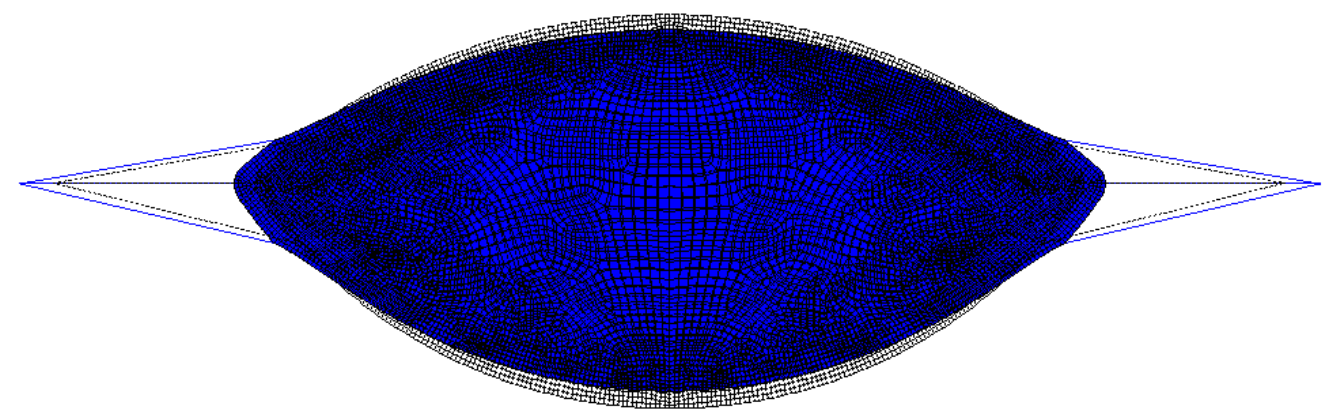

Figure 5.12: Finite element solution of accommodation model. Blue represents deformed lens, shading represents original version. 
Material properties of the crystalline lens from Brillouin microscopy and numerical

simulations

\subsubsection{Model sensitivity}

Additional simulations of the accommodation model were done in order to evaluate the model's sensitivity to other parameters. Changes of 50-100\% in magnitude of capsular thickness, capsular Young's modulus, and zonule spring constant were not found to have a substantial effect on the accommodative amplitude $(<5 \%)$.

\subsection{Limitations of Brillouin elasticity models for crystalline lenses}

Although an overall description of the lens stiffness can be obtained from reverse modeling of a compression test, there is no unique solution for two independent unknowns. The reverse modeling procedure was found to be successful to retrieve stiffness values, although it is sensitive to the shape of the object being compressed.

The results from the accommodation finite element model show that elasticity maps where the nucleus has a higher stiffness than the lens cortex yield a much lower accommodation range than elasticity maps where the nucleus has a lower stiffness than the cortex. The effect is much larger than the variations that can be expected from model sensitivity and from potential differences between the overall stiffness of the models being compared. Additionally, varying the gradient parameter $k$ shows that the accommodation range increases with $\mathrm{k}$ when $\mathrm{E}_{\mathrm{n}}>\mathrm{E}_{\mathrm{s}}$ and decreases with $k$ when $\mathrm{E}_{\mathrm{s}}>\mathrm{E}_{\mathrm{n}}$.

Given the amount of data and the limitations inherent to building accommodation models it would be premature to make strong assertions about the Brillouin elasticity models. If the crystalline lens is modeled as an elastic solid, the change in accommodative amplitude obtained from the simulation is more influenced by the spatial distribution of material properties than by the overall stiffness. The influence of this distribution appears to be the determinant factor in how the model performs. This suggests that, if the deformation of the real crystalline lens is that of an elastic solid, its behavior is unlikely to be accurately described by an elasticity model where the center, or nucleus, is stiffer than the periphery, or cortex.

The accommodation model's results are lower than those obtained by Burd. The maximum change in power found was $6 \mathrm{D}$, whereas Burd's paper is closer to $7.5 \mathrm{D}$. The material gradient models used are a likely cause; Burd's model uses discrete nucleus and cortical regions, with the nuclear region covers a smaller percentage of the overall 
volume and being offset along the equator. This further concentrates the deformation in the central region of the lens, with the likely effect of flattening the central surfaces further and thus yielding increased accommodation amplitude.

The lack of experimental deformed geometric parameters is a limitation of the model. It would be ideal to be able to compare the results from the numerical model to either an objective power measurement or to experimentally retrieve deformed surfaces and thickness.

The compression test is unable to retrieve multiple mechanical parameters using only the variables of displacement and force. One possible solution is to use additional data that can be retrieved from the compression experiment. Reilly et al, who reached the same conclusion about retrieval of multiple parameters, attempted an elastographic method, using images taken from a compression test and used the deformed shape of the lens as an input. In their work, however, the stiffness of the internal part of the lens is described by a single parameter (the $2^{\text {nd }}$ parameter they retrieve is the capsular stiffness). It is unclear whether two independent parameters describing the lens' internal contents and additional capsular parameters would yield a unique answer with this extra information.

If, as the results of this work suggest, the Brillouin elasticity models do not sufficiently describe the mechanical behavior of the crystalline lens, it's worth considering why. The crystalline lens has high water content (at least 67\%), and as such, its bulk modulus is much larger than its shear and Young's moduli. It is possible, then, that the spatial variations in bulk modulus do not reflect a similar spatial variation in the shear modulus. The spatial distributions found by Brillouin microscopy resemble both the gradient refractive index of the lens and the water content gradient (all of which are related). It does not appear that these properties determine the lens' response to stretching/accommodating. Brillouin microscopy is known to be affected by variations in water content, and methods have been suggested to separate the water content component from the tissue mechanics component (Scarcelli \& Yun, 2018; Shao et al., 2018).

Finite element models of the crystalline lens, as well as some mechanical studies of its properties, assume that the lens can be treated as an elastic solid. It is possible that this assumption masks the underlying phenomena in the lens. Limited work has been done 
Material properties of the crystalline lens from Brillouin microscopy and numerical simulations

on modeling the physiology of the crystalline lens, and it has been proposed that the lens' macrostructure (its central sutures) have an important influence in accommodation. This response would not be readily captured by high resolution Brillouin microscopy.

Both possibilities suggest that, although the information gathered by Brillouin microscopy is accurate, it might have limited usage as a diagnostics or research tool for presbyopia.

\subsection{Conclusions}

The accommodative amplitude of crystalline lenses modeled with a Brillouin elasticity map $\left(E_{n}>E_{s}\right)$ was found to be lower than the accommodative amplitude of lenses modeled with the opposite material gradient $\left(E_{s}>E_{n}\right)$. Under standard modeling assumptions, the ratio of nuclear to cortical stiffness has a larger effect on the accommodative amplitude than the aggregate stiffness. The findings suggest that Brillouin elasticity data from crystalline lenses might not fully describe the lens' behavior under standard loading conditions.

Understanding the mechanical properties of the crystalline lens in the young eye is important as an inspiration for new approaches to the restoration of accommodation in the presbyopic eyes, such as accommodating intraocular lenses. The next chapter presents the opto-mechanical design and evaluation of a novel accommodating IOL. 


\section{Design and ex situ performance of an accommodating intraocular lens}

\subsection{Introduction}

The most widely accepted theory of presbyopia (described in Section 1.2.4) is the lenticular theory, which states that changes in the crystalline lens are responsible for the loss of accommodation amplitude. There is evidence that older crystalline lenses exhibit increased stiffness (Charman, 2008; R.F. Fisher, 1971; Weeber et al., 2007; Wilde et al., 2012), and that the force required to deform ex vivo crystalline lenses is larger for aged lenses (Manns et al., 2007). The ciliary muscle, meanwhile, is believed to retain all or most of its functions (Pardue \& Sivak, 2000; Stachs et al., 2002; Tabernero et al., 2016).

Treatment of presbyopia largely consists of near vision aid such as spectacles. Multifocal corrections, such as multifocal contact lenses and multifocal IOLs, have also been proposed. Although these corrections have made it to market, their optical methods inherently result in reduced contrast and patients often report optical phenomena such as glaring and halos (Alio et al., 2017; Salerno et al., 2017). Dynamic proposals are less common and have not yet reached the market. An overview of these proposals can be read in Chapter 1.2.5.

Deformable AIOLs have been proposed, but not yet made it to market or resulted in published data on their mechanism of function (Sheppard et al., 2010). These type of lenses require some mechanism of engagement between the AIOL and the accommodative apparatus. Some proposals include the natural fibrotic process (McLeod, 2006), biocompatible adhesives (Agarwal et al., 2008), or mechanical attachments (Peng et al., 2003). Except for the Z lens capture haptics (Beer, 2015), to our knowledge these mechanisms have not been materialized.

At the time of writing, there is limited published work on experimental evaluation of AIOL concepts or fully implemented designs. Though various studies on AIOL performance have been conducted, these are largely focused on visual outcomes in patients (Ang et al., 2013; Lan et al., 2017; Marques \& Castanheira-Dinis, 2014; Tomás-Juan \& Murueta-Goyena, 2015). As previous cases show, visual outcomes can 
be affected by optical phenomena unrelated to dynamic accommodation (Pérez-Merino et al., 2014).

The VioBio research group has previously proposed and demonstrated a photochemical method for bonding hydrophilic polymers, such as those used in IOLs, to capsular tissue. The method is Rose Bengal Photobonding, described in - and demonstrated with silk in Chapter 4. Using the same photosensitizer and green light source, a hydrophilic polymer sample stained with Rose Bengal and in contact with capsular bag tissue can be quickly (5-10 min) bonded to the capsular bag, with recorded maximum forces higher than those found in the accommodative apparatus. The efficacy and viability of this method to bond hydrophilic polymer strips and entire commercially available IOLs to the capsular bag has been demonstrated, both ex vivo (Marcos et al., 2015) and intraocularly, in a porcine eye model, following cataract surgery procedures (AlejandreAlba et al., 2018).

This technique, which allows for the engagement of an IOL within the accommodative apparatus, much like the real crystalline lens, can be the viable mechanism of force translation into an accommodating lens. By photobonding the AIOL in the desired area of engagement, the need for full capsular bag integrity is mitigated. The VioBio research group has proposed an AIOL concept, which relies on this method of force translation to deform the surfaces of a soft optic, mimicking the real crystalline lens' changes upon accommodation, in order to obtain a change in power. This is done by bonding an AIOL with transverse, radially symmetric lens haptics of approximately 1 $\mathrm{mm}$ in height, to the equatorial capsular region (Dorronsoro et al., 2017).

This chapter evaluates the viability of this accommodating lens concept. Optomechanical models consisting of a finite element component and an optical component were built to evaluate the accommodative amplitude of the AIOL in various conditions. An accommodation system, consisting of three separate optical and mechanical platforms, was built in order to evaluate the physical and optical performance of an AIOL prototype. The results of this experimental work were contrasted with the results from the experimental platform. 


\subsection{Accommodating Lens}

The proposed design consists of two elements. The first is a deformable optic with eight haptics. The external surface of the haptics is curved and shaped to resemble the curvature of the crystalline lens' equator. It is this external surface that is in contact with the crystalline lens' capsule. The haptics contain Rose Bengal, which is deposited onto the surface as part of the manufacturing process, or manually applied before implantation. These haptics are bonded to the equatorial region of the capsular bag, allowing the ciliary muscle to apply a radial force to the lens through the capsular bag. In response, the optic deforms, with a subsequent change in optical power. The second element is a thinner optic, engaged to the deformable component. The optical power of this element remains constant, and is selected to correct for the individual patient's refractive error. A representation can be seen in Figure 6.1.
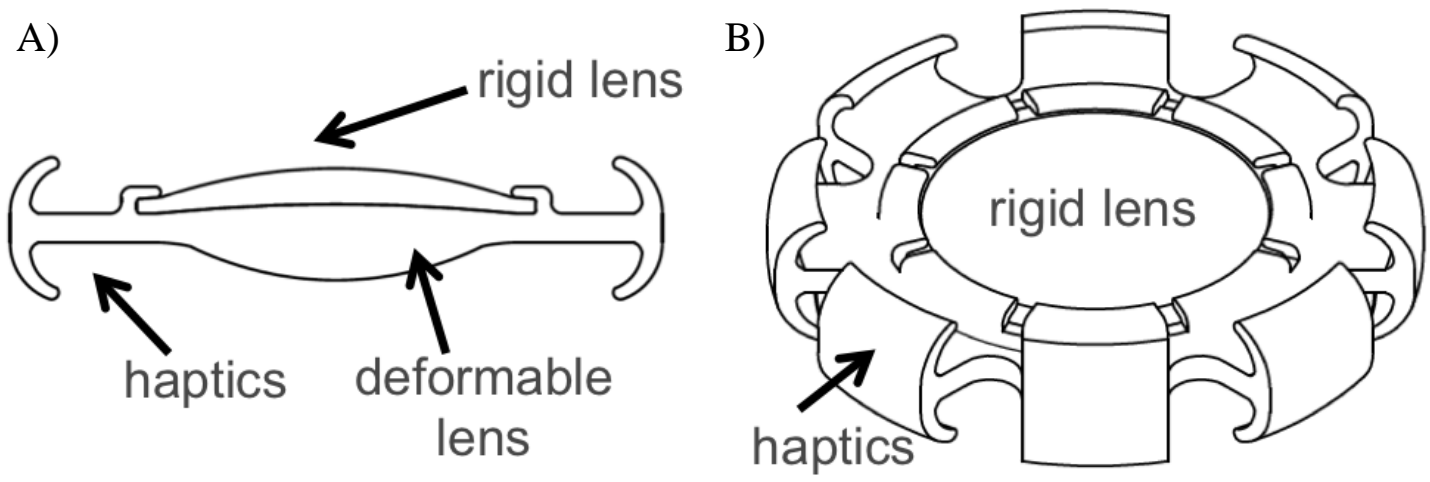

Figure 6.1: AIOL concept. A) cross-sectional view. B) 3D isometric view.

\subsubsection{Prototype}

AIOL prototypes were manufactured by Universidad del Norte (Barranquilla, Colombia) in order to evaluate in an experimental platform. A representative prototype is shown in Figure 6.2. The prototype design covers only the deformable component of the AIOL, as it is intended primarily to assess the response to loading. Therefore, no second optic or engagement system between first and second optic were manufactured.

The optical region of the prototype has an overall diameter of $5 \mathrm{~mm}$. The lens has 8 haptics, which extend $6 \mathrm{~mm}$ outward, for a total diameter of $11 \mathrm{~mm}$. The haptics in this prototype were designed to be fully flat, in order to clamp them onto the flat fixation shoes of the mechanical stretcher. The central thickness of the optical region of the prototype is $1.433 \mathrm{~mm}$, and the anterior and posterior radii of curvature are $12 \mathrm{~mm}$ and 
$5 \mathrm{~mm}$. The geometry was selected partly based on input from simulations, but with increased thickness and curvature in order to improve manual handling of the prototype and ease the quantification of geometric parameters in the OCT system.

The material used for the prototype was the Contaflex Hefilcon material, which has a refractive index of 1.42 . The material has a slightly blue tint.

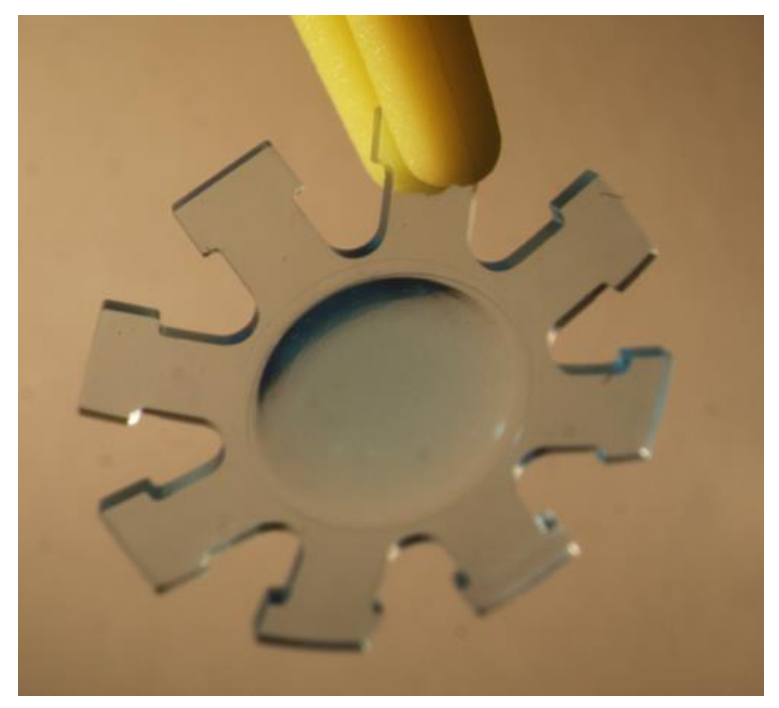

Figure 6.2: AIOL prototype.

\subsubsection{Material evaluation $\&$ constitutive models}

A study of the mechanical properties of transparent, hydrophilic polymers was conducted in order to characterize them for the purposes of finite element modeling.

A series of hydrophilic materials produced by two IOL and contact lens material manufacturing companies (Contamac and Vista Optics) were procured. Both companies provided samples of materials in their intraocular (water content $=25 \%$ ) and contact lens $($ water content $=35-50 \%$ ) ranges. The materials evaluated were Vistaflex $45 \%$, Hefilcon $42 \%$, Polymacon $38 \%$, IOSoft $26 \%$ and Contaflex 26\%. The materials were provided in the standard manufacturing shape, which is a disc of 14.5 to $16 \mathrm{~mm}$ in diameter and 2.5 to $5 \mathrm{~mm}$ in height. The cylindrical samples were cut using a diamond wire saw, into a rectangular shape of approximately 0.5 millimeters in thickness, 11$12.5 \mathrm{~mm}$ in length, and 2.5-5 $\mathrm{mm}$ in length (dependent on the initial height of the disc. The samples were then left to hydrate in water, for 24 hours. Hydrophilic polymers change their dimensions upon hydration (their radial expansion coefficients range from 1.15 to 1.27 for the materials evaluated). 
The mechanical properties of the materials were evaluated with a uniaxial extensiometry test. The uniaxial testing was done at the Universidad Politécnica de Madrid's Centro de Tecnología Biomédica. An Instron 5543A device was used, described in Section 2.1.4.

Before any measurements are done, a calibration is required, in order to quantify the force that water exerts on the measuring arm due to the volume it displaces. This push results in a positive force that varies with the position of the arm and must be subtracted from experimental measurements. In order to measure this force, the clamps were mounted on the arms and the distance between the two clamps is set to $8 \mathrm{~mm}$. The plastic chamber was fastened and filled with water. Then a uniaxial measurement was recorded for the full length of the movement of the arm.

The dimensions of each sample of material were measured before each experiment. The sample was then placed between the two clamps, and compressed until fixed. Care was taken to maintain a consistent distance of $8 \mathrm{~mm}$ between the two clamps. The clamps were mounted onto the Instron device, inside the bath. The bath is filled with water. The sample was allowed to rest in immersion for 15 minutes in order to rehydrate it (as a precaution from minor hydration loss during the clamping process). The tensile test measurement began after this point. After completion of the measurement, the bath was emptied, the clamps were removed, and a new sample was mounted.

Five samples of each material were measured. Five hydrophilic materials were measured in total. Previous data from the material manufacturer indicated that the material's viscoelastic response was limited within a reasonable range of deformation rates, and that the elastic behavior of the material is unaffected by a normal range of water temperature. The tests were done at a temperature of $20^{\circ} \mathrm{C}$. A verification of an influence of the rate was done using a sample from one material and no effect larger than the standard experimental variations within samples were found. Therefore, all tests were done for a fixed rate of $0.2 \mathrm{~mm} / \mathrm{s}$.

The data was then processed. The calibration force was subtracted from the recorded force in order to obtain the actual force applied to the sample. The force and displacement were plotted in order to find the true beginning of the uniaxial stretch (as the initial mounting often had a small amount of slack). This point was selected as the starting point; the force at this point is subtracted from all other values to set zero. 
Engineering stress is obtained by dividing the force by the initial cross-sectional area. The deformation was defined by the stretch ratio, taking the length at force zero as the initial size of the sample.

Hyperelastic, isotropic material models were used to fit the uniaxial stress-strain data. Different material models (Neo-Hookean, Mooney-Rivlin and Yeoh 2- and 3parameter models) and their suitability was evaluated. The models are described in Section 1.5.2 and the fitting equations are described in Section 1.5.3. The BakerEricksen inequalities are used to constrain the models, by ensuring that $\partial W / \partial I_{i}$ is positive for a large range of stretch ratios $(\lambda=3)$.

\subsection{Numerical models of AIOL}

Finite element models were developed in ANSYS Workbench (described in Section 2.2.1) to evaluate the AIOL's response under loading. Two separate models were developed, following the same procedure. The first model simulates the ex vivo performance of the AIOL prototype in the bench testing platform. The mesh of this model is presented in Figure 6.3A. The second model evaluates AIOL performance as a function of material and design parameters. The mesh of this model is presented in Figure 6.4.

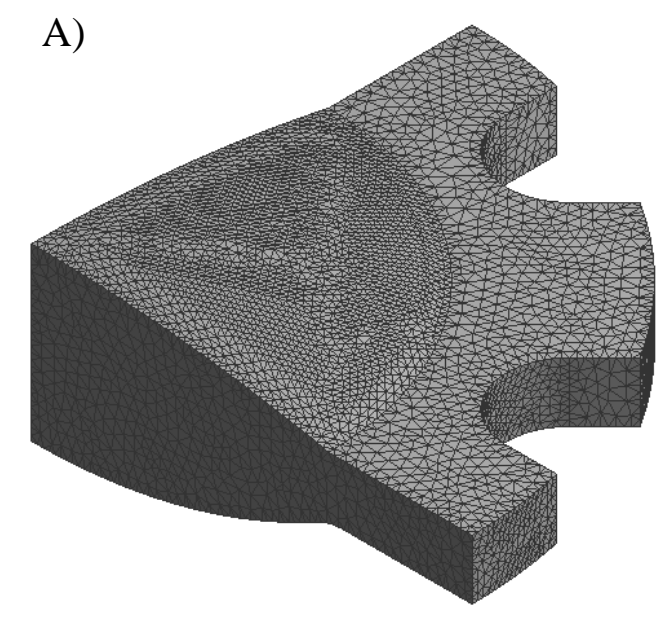

B)

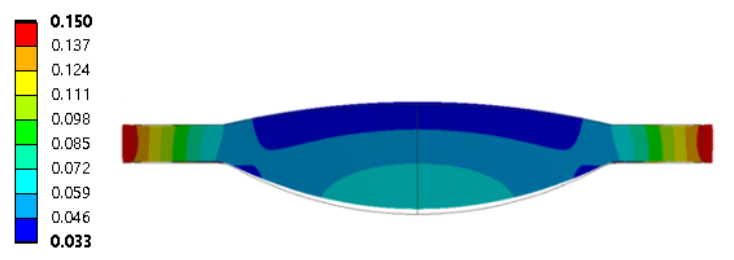

C)

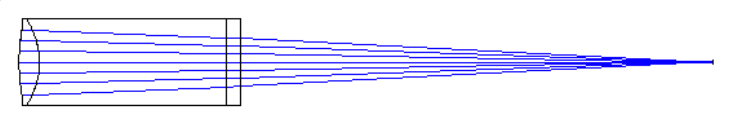

Figure 6.3: A) Finite element model of AIOL prototype. B) Displacement of AIOL prototype in model (units: mm). C) ZEMAX model of lens in accommodation system's water chamber-

Finite element simulation is a processor-heavy task. Simplification of the models, in order to reduce processing time, while still yielding accurate and usable results, is a necessity. Symmetry is the most standard form of simplification. The AIOL modeled is 
a symmetrical lens, with eight haptics evenly spaced. The maximum symmetry available is separating the lens in eight parts. However, this requires that other symmetrical lens components (namely the system that may hold the rigid and deformable lenses together) align symmetrically with the haptic symmetry planes. For the purpose of these simulations, the geometric model consists of one fourth of the total volume of the AIOL, with symmetry on the $\mathrm{X}$ and $\mathrm{Z}$ planes.

The haptics of the AIOL were not modeled fully. Instead, the haptics extend $2 \mathrm{~mm}$ and $0.75 \mathrm{~mm}$ from the $5 \mathrm{~mm}$ optical region of the AIOL in the first and second models, respectively. In the first model, this simplification reflects the clamping of the prototype's flat haptics. In the second model, the simplification is done for two reasons. The first is that it saves computational time by not having to describe the entirety of the haptic, especially the larger region that is photobonded to the lens' capsule. The second is that it is not possible to accurately model the full accommodation mechanism, at least not at the same level as the AIOL itself can be modeled. A study by Glasser and colleagues suggests that changes in surface shape and power of the biological crystalline lens can be represented (and thus modeled) with an equatorial force, without accounting for additional forces (Glasser \& Wendt, 2013). Although some work on finite element modeling uses zonulae and ciliary body to apply the accommodation force (such as the work presented in Chapter 0), the parameters used to model the zonulae are retrofitted to achieve expected changes, an approach not suitable to the needs of this work.

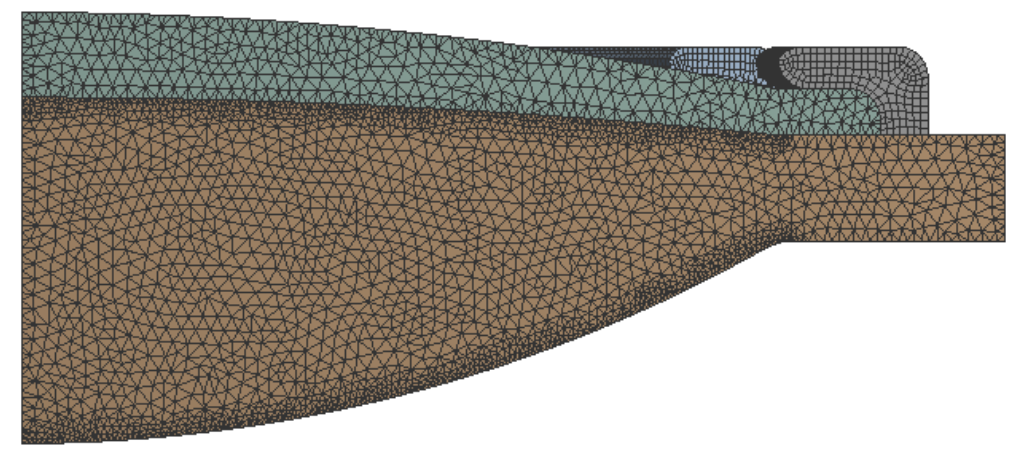

Figure 6.4: Finite element model of AIOL for in vivo performance.

The AIOL was modeled using the SOLID187 element type. This is a 3-dimensional, 10node tetrahedral solid element. The element is suitable for the purpose of the model due to the following characteristics: allows for irregular meshing (for complex geometries), 
and has hyperelastic and finite strain capabilities. In order to find the appropriate element size for the model, a sequence of simulations were made with decreasing element sizes, and the radii of curvature and thickness were evaluated. Figure 6.5 shows the result of these simulations. The values for radii and thickness stabilize at an element size of $0.05 \mathrm{~mm}$. This value was selected as the element size for the remainder of the simulations.

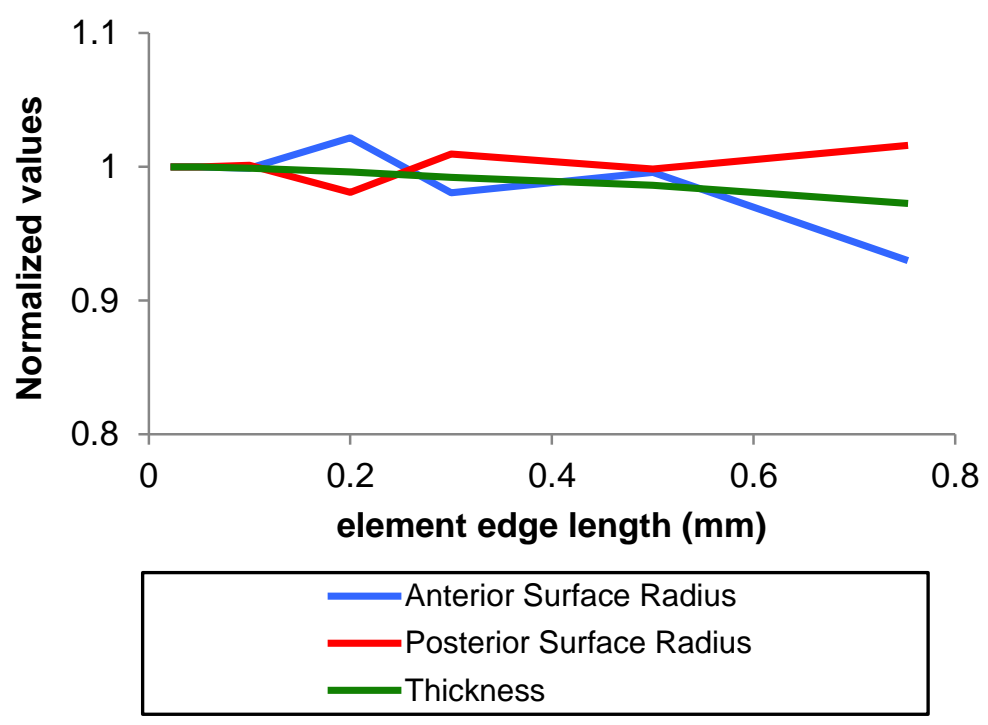

Figure 6.5. Calculated curvature and thickness values and variation with element size. Results converge at 0.05 mm.

After meshing, the boundary conditions are applied to the model. There are two boundary restrictions. The first is a cylindrical support - applied to the external surface of the haptics. This ensures that the nodes on this surface remain on the same axial coordinate (in a cylindrical coordinate system) throughout the simulation. The second is the load, which is also applied onto the external surface of the haptics. In the ex vivo performance model, a pressure load equivalent to $0.6 \mathrm{~N}$ was used. In the in vivo model, a pressure load equivalent to $0.08 \mathrm{~N}$ was applied. This load is consistent with values found in the literature (referenced in section 1.2.3 of this thesis) and used in the crystalline lens accommodation model presented in this thesis (Section 0).

Once the solution of the numerical model was obtained, the geometric changes were evaluated. The three main variables that were evaluated were the radii of curvature, and the thickness of the lens. The thickness of the deformed AIOL was evaluated by subtracting the coordinates of the displaced nodes lying at the center of the anterior and posterior surfaces (at $\mathrm{x}, \mathrm{z}=0$ ). 
For the evaluation of the deformed surfaces, a number of different methods were used. In order to quantify the changes in the surfaces within the ANSYS software, the code that adapts the Pratt method (described in Section 0) was implemented into this finite element model. The input were the nodes along a 2 dimensional cross-section at $\mathrm{z}=0$. In order to ensure the resulting circle was centered at 0 , the data points were mirrored.

Additionally, the position of nodes lying on the anterior and posterior surfaces of the AIOL model were extracted and evaluated with a custom MATLAB code in order to do a 3-dimensional reconstruction of the surface. The code evaluates the surfaces as conics (in terms of a radius $\mathrm{R}$ and an asphericity $\mathrm{Q}$ ) and as 23-term Zernike surfaces.

\subsubsection{Optical models}

A model of the experimental conditions was designed in ZEMAX (described in Section 2.2.3). It is an axisymmetric model with surfaces representing the water, AIOL, air, and CCD camera used experimentally. This first optical model was used to evaluate the results from the first finite element model, in order to simulate the AIOL's performance ex vivo. The change in focal length of the system after loading was obtained from the model. The model is represented in Figure 6.3C.

A second optical model was developed to evaluate the AIOL's design and material parameters in vivo. This model represents the eye and replaces the crystalline lens with the accommodating IOL. To build the model, information on the geometrical and optical parameters is required. Figure 6.6 shows a schematic representation of the optical model and its definitions.

The first refractive element is the cornea, defined by two surfaces (anterior and posterior curvature of the cornea) and a refractive index. The anterior and posterior curvature of the cornea can be measured through Scheimpflug imaging or OCT. Hernandez et al measured corneal radii using a commercial Scheimpflug imaging system and found average radii of 7.64 and $6.29 \mathrm{~mm}$ (Hernandez et al., 2015) for 16 subjects of a mean age of $24.5 \pm 3.3$ yrs. The refractive index of the cornea is established in the literature as 1.376 (Levin et al., 2011).

For the model, the values used were: corneal radii of curvature of 7.70 and $6.40 \mathrm{~mm}$, central thickness of $0.5 \mathrm{~mm}$, and refractive index of 1.376 . 
The anterior chamber depth (ACD) is defined as the distance between the posterior surface of the central cornea and the anterior surface of the crystalline lens. ACD has been previously measured using OCT systems. Hernandez and colleagues, using a spectral-domain OCT system, found a mean ACD value of $3.26 \mathrm{~mm}$ (Hernandez et al., 2015). Pérez-Merino and colleagues, using a similar system, found a mean ACD value of $3.19 \pm 0.21 \mathrm{~mm}$ in a maximally accommodated state of 6D (Pérez-Merino et al., 2015). With the same system, a mean ACD value of $2.96 \mathrm{~mm} \pm 0.21 \mathrm{~mm}$ was obtained in a separate work (Martinez-Enriquez et al., 2017). For this model, the value used for the ACD was $3.1 \mathrm{~mm}$. This is set as the distance between the cornea and the iris. The refractive index of the anterior chamber (consisting of aqueous humor) was defined as 1.336 for the model.

As the crystalline lens contents are replaced by the AIOL, the relevant parameter is the thickness of the lens (LT). Perez-Merino and Martinez-Enriquez found a mean LT of $4.12 \pm 0.2 \mathrm{~mm}$ and $4.134 \pm 0.2 \mathrm{~mm}$, respectively was found for the maximally accommodated state (Pérez-Merino et al., 2015). Hernandez and colleagues found a mean value of $3.634 \pm 0.174 \mathrm{~mm}$. Rosen and colleagues, in a study of in vitro crystalline lenses, were able to fit a relationship between lens thickness and age as follows: $0.0123( \pm 0.003) \times$ Age $+3.97( \pm 0.16) \mathrm{mm}$ for lenses of subjects aged 20 to 99 (Rosen et al., 2006). An OCT study by Richdale et al found a relationship of $0.021 \mathrm{x}$ Age $+3.14 \mathrm{~mm}$ for subjects aged 36 to 50 (Richdale et al., 2008).

A relevant parameter related to the lens thickness is the equatorial plane position (EPP), defined as the distance between the anterior lens surface and its equatorial plane. As the lens' anterior and posterior surfaces differ substantially, the EPP is typically located closer to the anterior surface than to the posterior one. Martinez-Enriquez found an EPP value of $1.61 \pm 0.10 \mathrm{~mm}$, and Rosen found an age-EPP relationship of $0.0049 \mathrm{x}$ Age + 1.65. For this model, the $1.61 \mathrm{~mm}$ EPP value was chosen. The AIOL's deformable component is aligned with this plane, with the rigid component positioned in front. This means around $40 \%$ of the deformable component's thickness (around 0.4-0.5 mm on average), plus the rigid component (of varying thickness, but approximately $0.5 \mathrm{~mm}$ ) lies between the iris and the EPP.

The last relevant distance is the vitreous depth. Since the vitreous depth is measured from the posterior surface of the crystalline lens, which is considerably thicker than the 
AIOL, this depth will not be used as the value in the model. Instead the vitreous depth is set as a function of the total depth of the eye, from the anterior surface of the cornea to the retina. A number of studies have found the axial length of the eye to be between 2225 mm (Bhardwaj \& Rajeshbhai, 2013; Dong et al., 2018; Kim et al., 2019). The model uses a value of $24 \mathrm{~mm}$. The refractive index of the vitreous humor, as with the aqueous humor, is 1.336. The parameter values used for the model are described in Table 6.1.

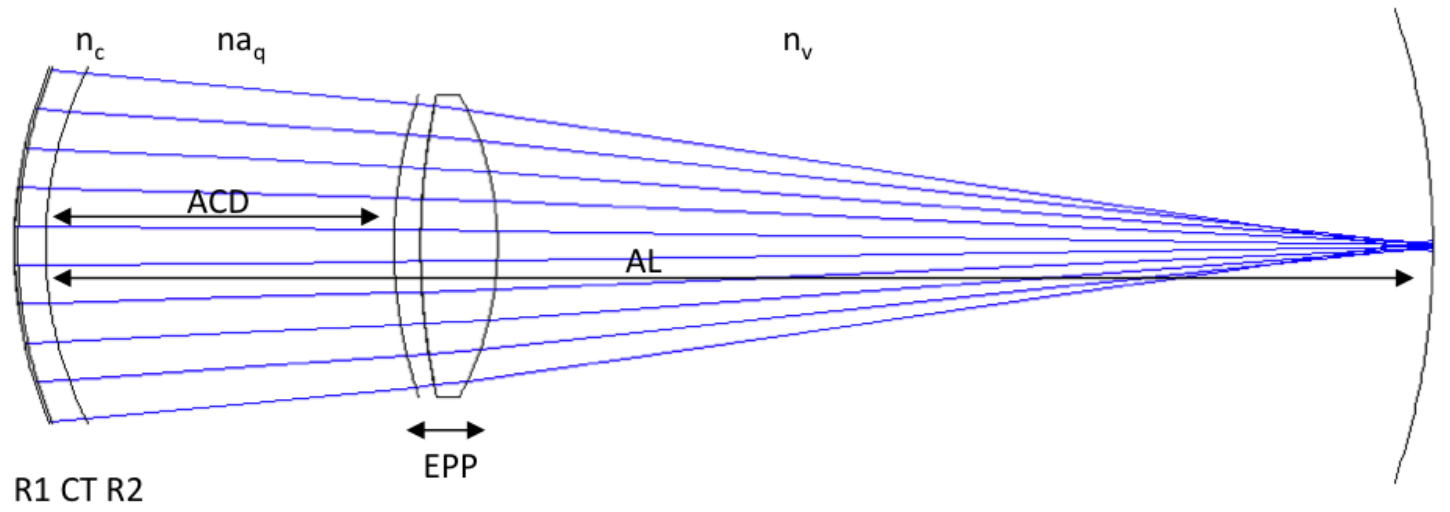

Figure 6.6: Schematic of optical model of eye and AIOL

\begin{tabular}{|cc|}
\hline Parameter & Value \\
Anterior corneal radius $(R 1)$ & $7.70 \mathrm{~mm}$ \\
Posterior corneal radius $(R 2)$ & $6.40 \mathrm{~mm}$ \\
Corneal thickness $(C T)$ & $0.50 \mathrm{~mm}$ \\
Corneal refractive index $\left(n_{c}\right)$ & 1.376 \\
Anterior chamber depth $(A C D)$ & $3.12 \mathrm{~mm}$ \\
Aqueous humour refractive index $\left(n_{a q}\right)$ & 1.336 \\
Equatorial plane position $(E P P)$ & 1.61 \\
Vitreous humor refractive index $\left(n_{v}\right)$ & 1.336 \\
Axial length $(A L)$ & $24 \mathrm{~mm}$ \\
\hline
\end{tabular}

Table 6.1: Optical model parameters

The analysis procedure was the following: nominal surface values for the surfaces of the AIOL are input into the ZEMAX optical model. The object distance is calculated by setting as variable to be solved. The deformed surface values are input into the model and the same procedure is followed. The inverse of the distances is taken as the lens power, and the difference between the two is taken as the change in power after deformation.

\subsubsection{Experimental models for opto-mechanical evaluation}

The accommodation system described in Chapter 2.1.1 was used in this work. The system is a combination of different optical and mechanical technologies that apply a 
load to an intraocular lens, quantify it, and characterize the optical and physical response to the load.

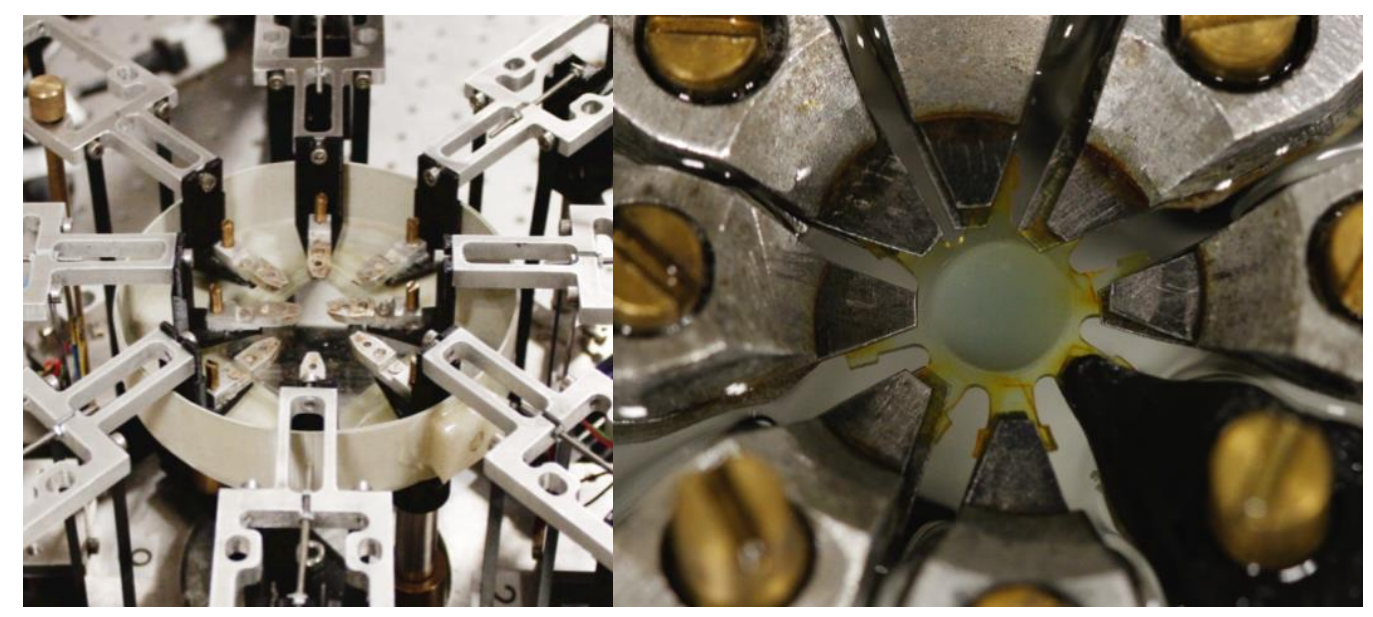

Figure 6.7: A) Radial stretcher system. B) Prototype clamped and mounted on stretcher.

\section{Radial stretcher}

The AIOL prototype was mounted onto the radial stretcher for extension. The system applies a fixed displacement and outputs the force required to achieve it.

\section{Laser Ray Tracer system}

The LRT system scans a circle of light onto the AIOL and records the image produced by the lens at different heights. With this information, the change in focal length in the system can be quantified.

\section{Optical Coherence Tomography system}

The OCT system images the AIOL before and after loading. The images can be used to create a 3-dimensional reconstruction of the AIOL. The surfaces and thickness of this reconstruction can be quantified using MATLAB algorithms.

\subsubsection{AIOL prototype evaluation: experimental protocols}

\section{Radial stretcher}

The mechanical stretcher's arms were aligned and the AIOL prototype was placed in their center, and then clamped with the eight fixation shoes. The immersion chamber was then filled with distilled water and the AIOL prototype was allowed to recover any lost hydration. A small initial force $(0.01 \mathrm{~N})$ was then applied to the AIOL to remove 
any potential slack from the mounting process. The mechanical stretcher is aligned to ensure the light from the laser ray tracing is symmetrical along the optical surface.

The structure for the experimental measurements is the following: Each cycle consists of five substeps (force applied) in which the AIOL is progressively stretched (after the first sub-step) by applying a consistent displacement on each of the 8 arms. The total force applied at the end of the final sub-step is approximately $0.55 \mathrm{~N}$. Then the prototype is returned to its initial deformation state, in order to begin a new cycle. Five total cycles are done for each device: laser ray tracing system, and OCT system. The cycles are not simultaneous; the laser ray tracing cycles are done first, and the OCT cycle afterward. The five total cycles taken constitute a trial.

\section{Laser Ray Tracer}

Each sub-step of the LRT system consists of a sweep of the CCD camera, for a total axial distance of $20 \mathrm{~mm}$, at a motor step size of $0.25 \mathrm{~mm}$. At each step of the motor, the camera captures a spot image of the light source going through the lens.

The images are then processed using a MATLAB code. The code converts each image into a binary image with two levels (white and black) based on a threshold value previously optimized for the camera's images. A histogram of the binary image is obtained and the pixel count for the white level is used to assess the total size of the spot of light. The spot size is plotted in terms of the motor position, and a polynomial fit of the relationship is found. The polynomial fit is evaluated at axial intervals of $0.1 \mathrm{~mm}$ and the minimum value is found. This axial value is selected as the focal length of the lens in the system. The initial focal length value is subtracted from all measurements. The measured focal length values were then compared to simulated focal length changes calculated from the ANSYS-ZEMAX model.

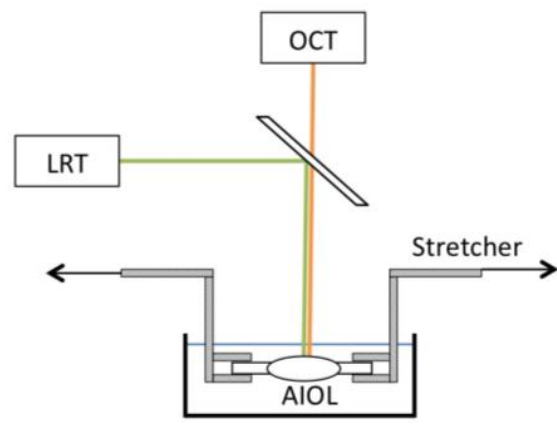

Figure 6.8: Schematic of lens evaluation in accommodation system. 


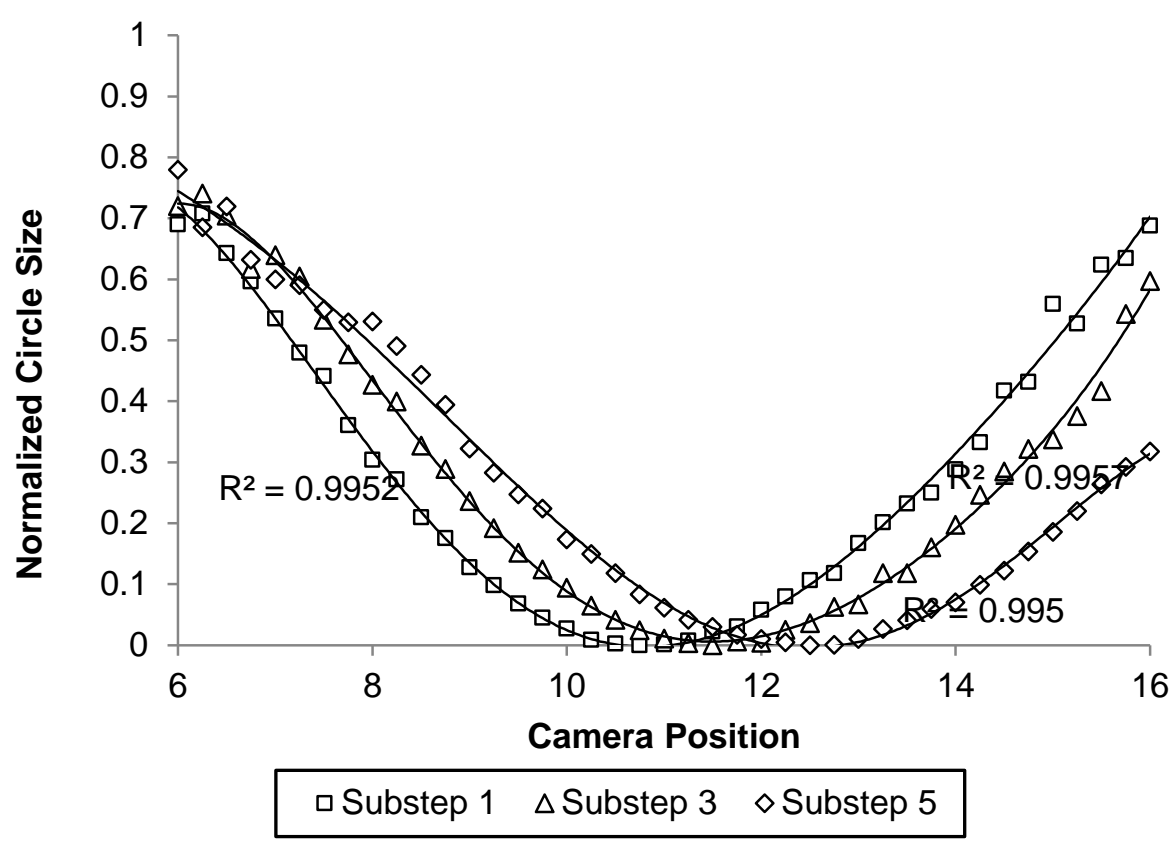

Figure 6.9: Processed LRT data

Figure 6.9 shows the intensity/spot size curves for substeps 1, 3, and 5 of a single cycle in a trial. It was found that, for a sweep of $20 \mathrm{~mm}$, the results for the spot size became noisier 5-6 mm away from the minimum spot size; this is due to the variations in intensity over distance being too large for the threshold method used. Therefore, a cutoff was established, and only the central $10 \mathrm{~mm}$ of the total $20 \mathrm{~mm}$ sweep were used for processing.

\section{OCT}

The OCT measurements use the same structure of 5 sub-steps and 5 total cycles. For each measurement, 300 B-scans, of 300 A-Scans per B-Scan, were obtained. The dimensions selected were $6 \mathrm{~mm}$ by $6 \mathrm{~mm}$ in $\mathrm{x}-\mathrm{y}$ plane; the depth of the system is $5 \mathrm{~mm}$ in the $\mathrm{z}$-axis. For OCT, additional trials were done for the AIOL in a reverse configuration (posterior surface facing up toward the rays). The images were then processed in MATLAB using custom-made algorithms developed by the VioBio lab. First, optical (produced by refraction caused by the optical surfaces) and fan (produced by the scanning architecture of the OCT system) distortion were corrected. The automatic surface segmentation is based on algorithms described previously. After obtaining a volume representation of the AIOL, a previously written MATLAB code was used to quantify the surfaces as spheres. 


\subsection{Results}

\subsubsection{Material evaluation \& constitutive models}

Figure 6.10 shows experimental results for the Hefilcon material. The results are consistent across samples, especially on the lower range of stretch ratio. There is much larger variation in the maximum elongation before break. As the samples were clamped rather than glued, and due to being flexible, it is likely that compressive forces at the edge account for early tensile break in some samples.

Figure 6.11 shows representative results for samples of the hydrophilic materials evaluated. The differences in elasticity between different types of materials can be clearly observed, with the intraocular lens materials (Contaflex, IOSOft) having higher stress upon deformation than the contact lens materials (Hefilcon, Polymacon, Vistaflex). The maximum stretch ratio for most materials was between 1.8 and 2.0, with the IOSoft material extending up to 2.5-3.0.

The experimental data was used to obtain the coefficient values for various constitutive models. The models studied were the Neo-Hookean, 2- and 3-term Mooney Rivlin models, and 2- and 3- term Yeoh models. In order to select the most suitable model, the constitutive models with the calculated coefficient values were used to evaluate an equibiaxial stress-strain relationship. The analysis showed that the stress response varied considerably for higher values of stretch ratio. Additionally, the uniaxial stressstrain response was separated in terms of its components $\left(\mathrm{C}_{10}, \mathrm{C}_{01}\right.$, etc.). What the results suggest is that, for the stretch ratio range of 1.0-1.5, 2- and 3-parameter models are not necessary to describe the shape of the stress-strain curve, and as such, these constitutive models were over-determined, and this over-determination causes problems in the response under other loading conditions (such as equibiaxial tension). 


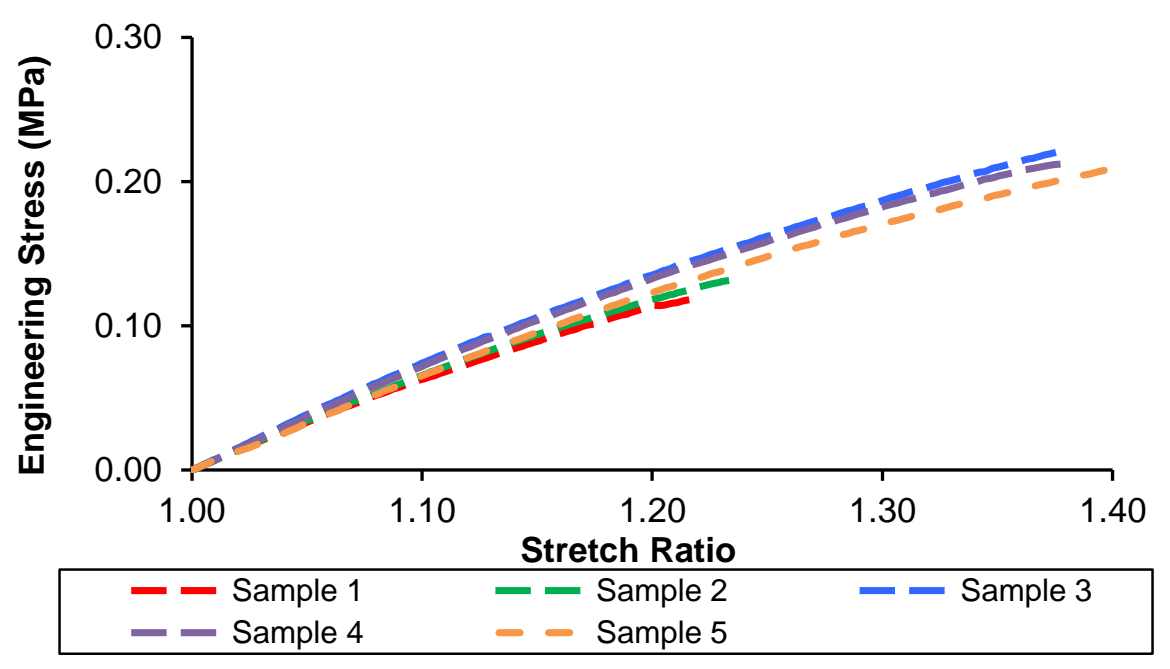

Figure 6.10: Stress-stretch ratio relationship for Polymacon material.

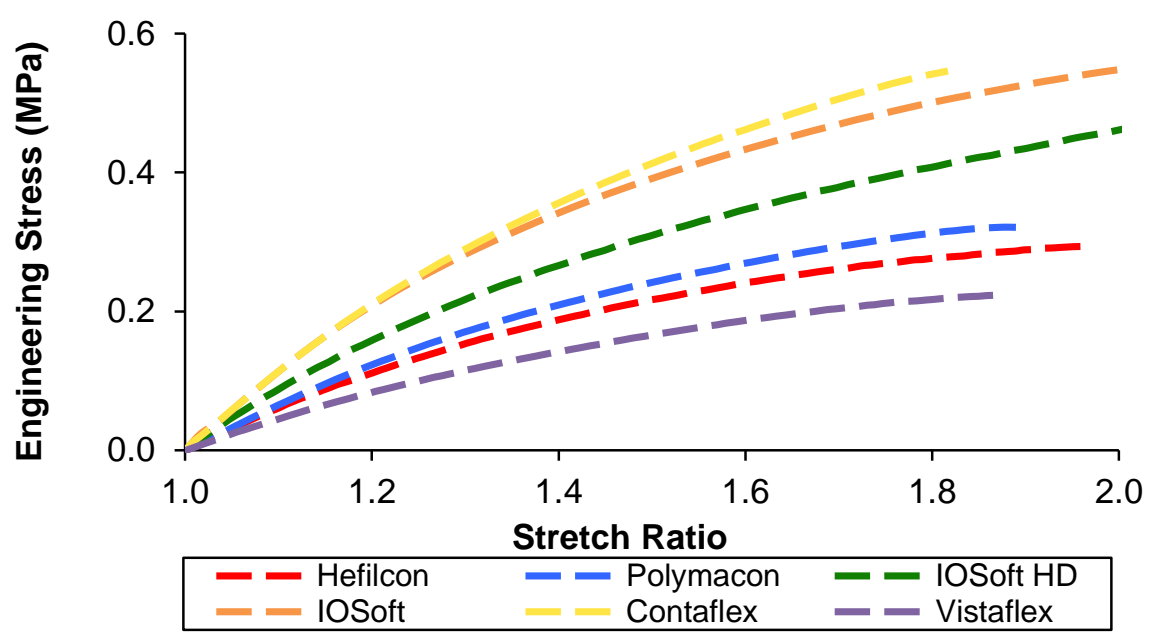

Figure 6.11: Representative stress-stretch ratio curves for all materials evaluated

The constitutive models were evaluated in the finite element model and the overall response is presented in Table 6.2. The strain values are the same for all models except the 3-parameter Yeoh model.

\begin{tabular}{|ccc|}
\hline Material Model & Maximum True & Maximum True \\
& Stress (MPa) & Strain \\
Neo-Hookean & 0.0909 & 0.1303 \\
Mooney-Rivlin 2-parameter & 0.0909 & 0.1301 \\
Mooney-Rivlin 3-parameter & 0.0910 & 0.1302 \\
Yeoh 2-parameter & 0.0909 & 0.1302 \\
Yeoh 3-parameter & 0.0919 & 0.1375 \\
\hline
\end{tabular}

Table 6.2: Maximum stress and strain for various constitutive models. 
Taking this into consideration, the Neo-Hookean model, with 1 coefficient, was chosen for all further material models. The stretch ratio range of 1.0-1.5 was used to obtain the material coefficient $\mu$. The calculated $\mu$ value is displayed in Table 6.3.

\begin{tabular}{|cc|}
\hline Material & Neo-Hookean coefficient \\
Vistaflex Advantage 49\% & 0.155 \\
Hefilcon 42\% & 0.217 \\
Polymacon 38\% & 0.257 \\
IOSoft 26\% & 0.388 \\
IOSoft HD 26\% & 0.3024 \\
Contaflex 26\% & 0.394 \\
\hline
\end{tabular}

Table 6.3: Neo-Hookean coefficient value for all evaluated materials

\subsubsection{Ex vivo optical changes upon accommodation}

Figure 6.12 shows the camera position in which the minimum spot size was found, as a function of the force applied to the AIOL. The absolute values of the minimum spot size distance (or focal length of the AIOL at a given stretching condition) show a standard deviation of $0.23 \mathrm{~mm}$. The majority of this deviation is related to the offset in individual cycles of measurement, due to variations in the initial focal length. If the position values for each cycle are zeroed at their starting position, the standard deviation drops to 0.071 $\mathrm{mm}$.

Figure 6.13 shows the predicted results (obtained from the opto-mechanical model) as well as the experimental results of the AIOL performance ex vivo as evaluated in the laser ray tracing system. Figure 6.13A represents experimental results for a circle of total diameter of $4 \mathrm{~mm}$, and Figure 6.13B represents experimental results for a circle of $4.5 \mathrm{~mm}$ diameter. Each step in each trial that is represented on the graph is the average of the 5 measurements obtained for that stretching force, and the error bars represent the standard deviation of the measurements. The average standard deviation of the change in focal length is $0.064 \mathrm{~mm}$. This deviation comes both from variations in the experimental measurements and from variations in the curve fitting done in postprocessing. The average standard deviation of the force is $0.0124 \mathrm{~N}$, from variations in the recorded force measurement for the same motor movement. 


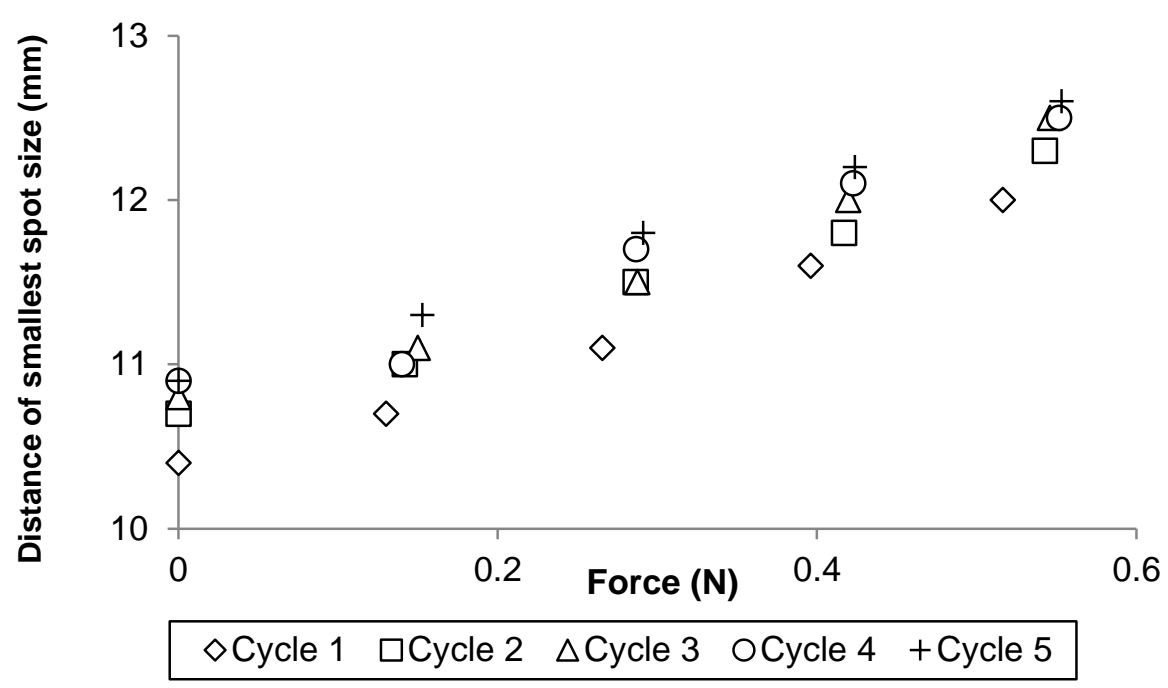

Figure 6.12: Representative trial in LRT system.

The slopes obtained for the linear fit of all data are $3.16 \mathrm{~mm} / \mathrm{N}$ and $3.55 \mathrm{~mm} / \mathrm{N}$, respectively, with $\mathrm{R}$ values of 0.978 and 0.973 . The predicted slopes are $3.0 \mathrm{~mm} / \mathrm{N}$ and $3.26 \mathrm{~mm} / \mathrm{N}$, showing a difference of $5 \%$ and $8 \%$ from the experimentally calculated values. In both cases, a y-intercept is found: $0.1 \mathrm{~mm}$ and $0.07 \mathrm{~mm}$. These intercepts suggest the first stretching of the lens does not follow the linear trend, which could be accounted by the experimental setup (movement or shifting of the lens during the first stretching movement). However, the values of the intercept are below both the resolution of the experimental measurements $(0.25 \mathrm{~mm})$ and that of the fitted curve $(0.1$ $\mathrm{mm})$. The significance is likely minimal. It was found that the value of this intercept is reduced when the lens is pre-stretched. The standard deviation of slopes per trial found was $0.072 \mathrm{~mm}$.

Overall, the experiments showed a strong correlation with the predicted behavior of the lens. 

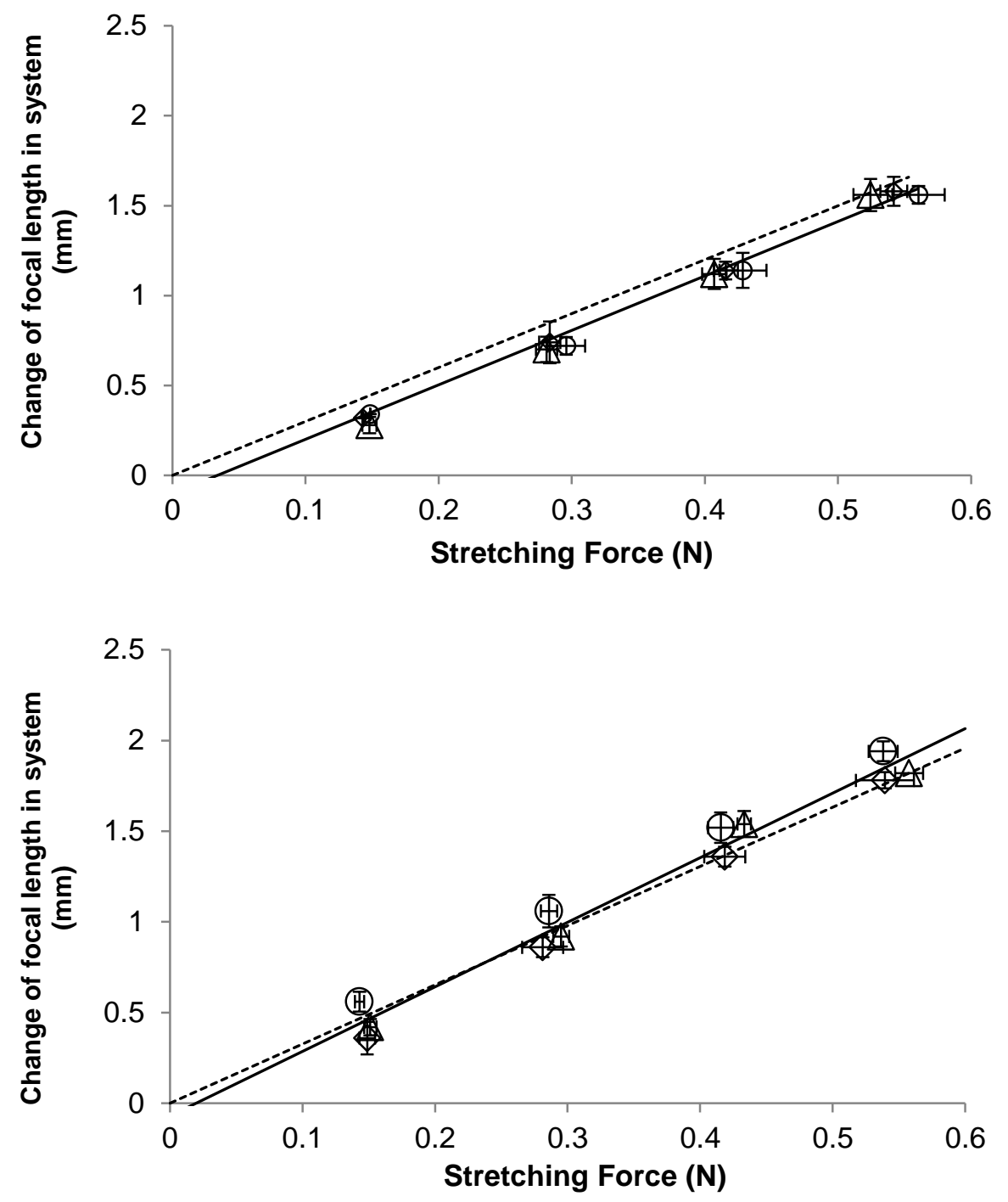

○ Trial $1 \quad \Delta$ Trial $2 \quad \diamond \quad$ Trial $3 \quad$ Linear Fit

Figure 6.13: Full results of LRT experiments for 4 and $4.5 \mathrm{~mm}$ spots.

\subsubsection{Geometry changes upon accommodation}

Figure 6.14 shows predicted (from opto-mechanical model) and evaluated (from OCT system) values for lens thickness, anterior and posterior surface radius with respect to stretching force. The figure shows results from trials with the anterior surface being the first surface, and for trials in which the posterior surface is the first surface. The second surface is reconstructed after correcting for optical distortion produced by the first surface. 

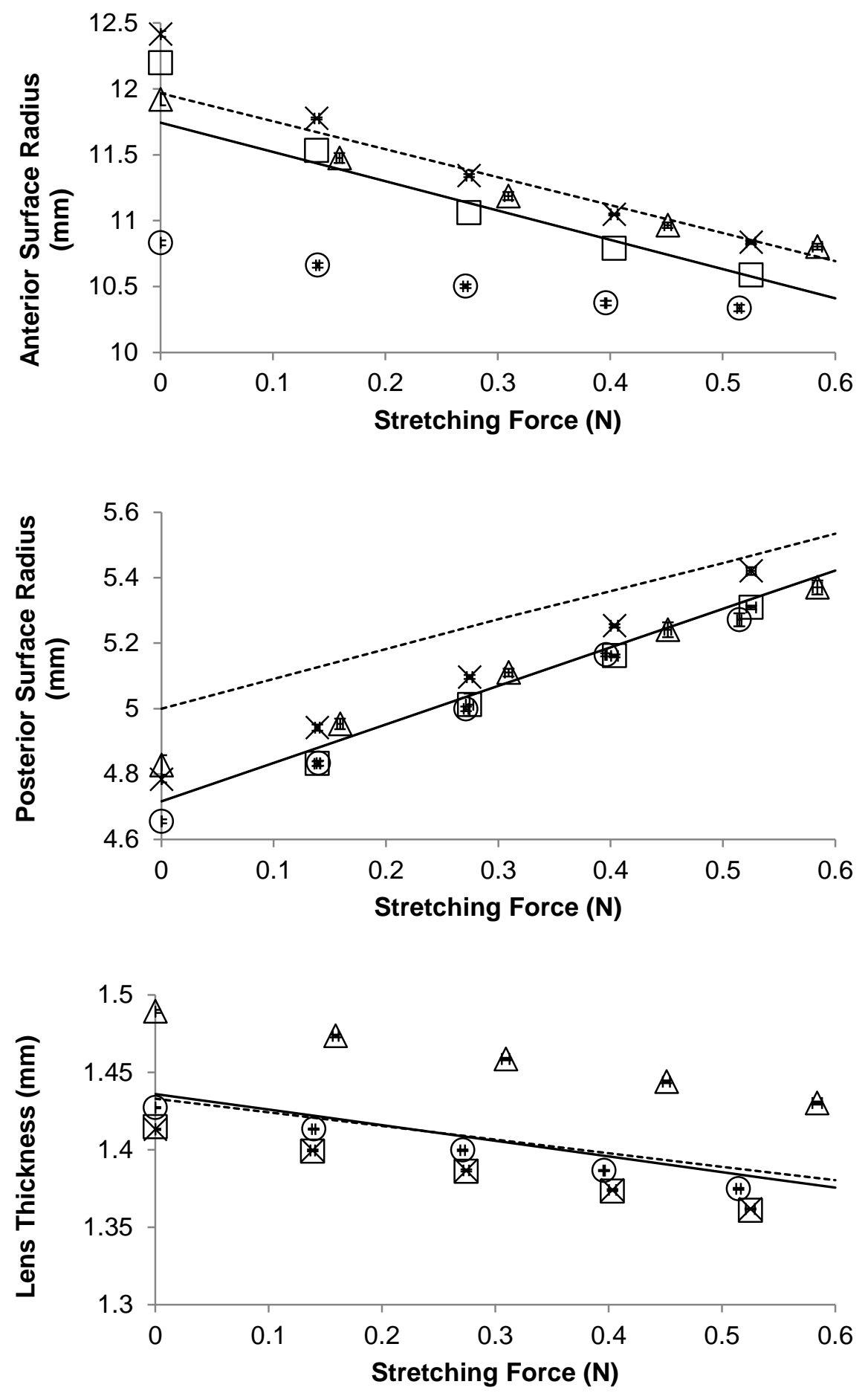

\begin{tabular}{|cccc|}
\hline$\times$ & Trial 1 (Post Up) & $\circ$ & Trial 2 (Post Up) \\
$\Delta$ & Trial 3 (Ant Up) & $\square$ & Trial 4 (Ant Up) \\
& Linear Fit & ------- Predicted Results \\
\hline
\end{tabular}

Figure 6.14. Results from OCT experiments and analysis. A) Anterior surface radius B) Posterior surface radius C) Lens thickness. 


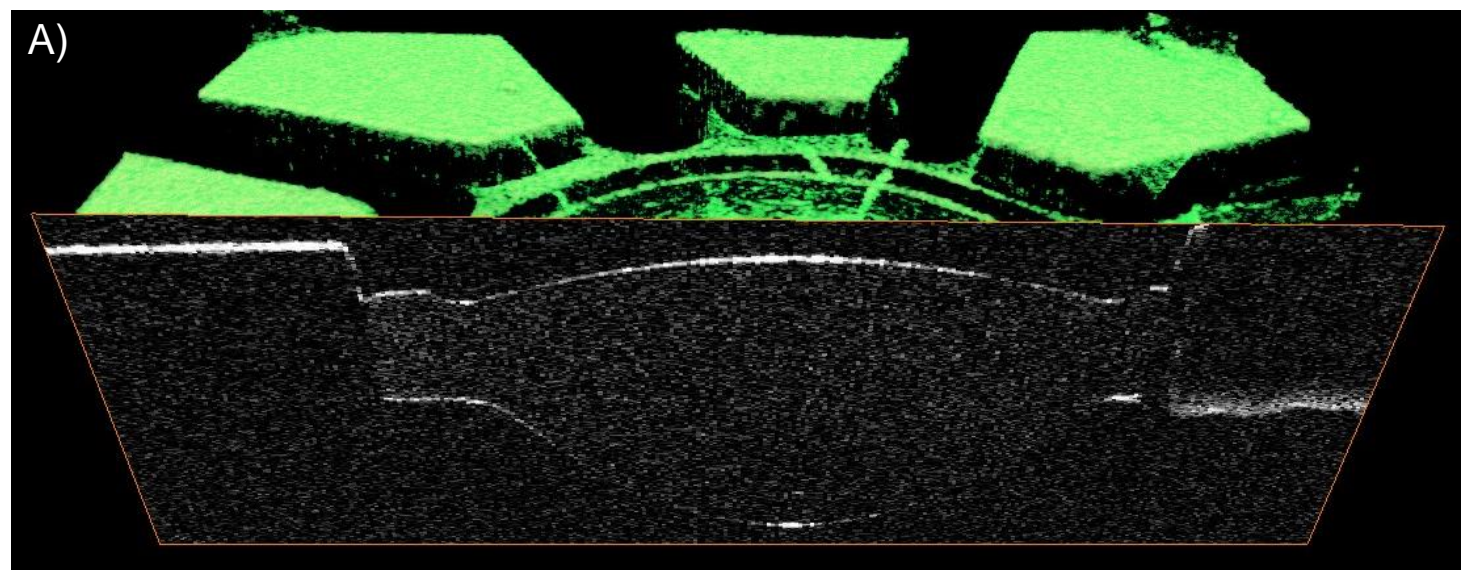

B)
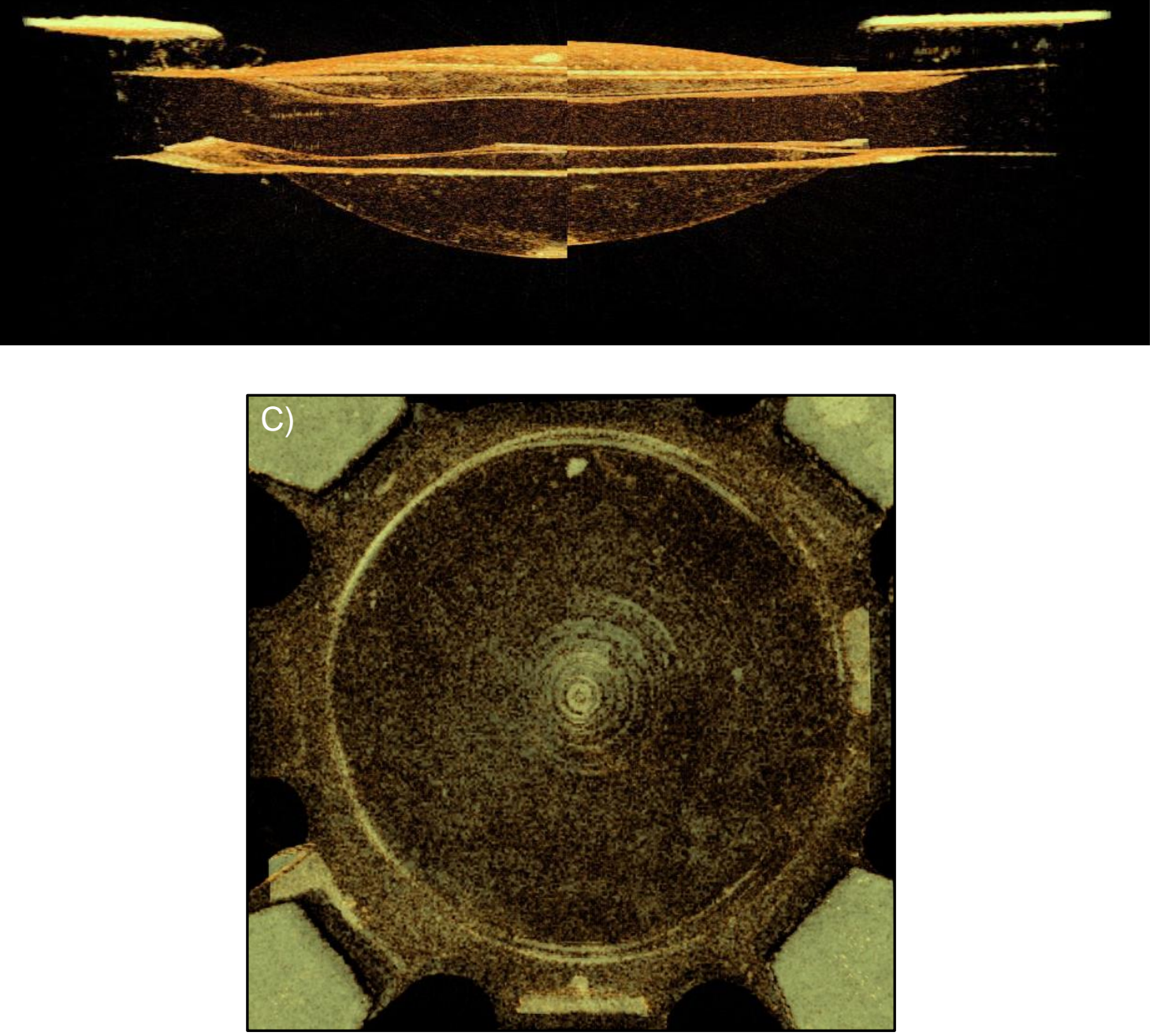

Figure 6.15: Reconstruction of OCT data. A) AIOL reconstruction with cross-sectional image of B-scan. B) Reconstruction of full volume in accommodated (right) and disacommodated (left) states. C) Top view of reconstruction. Stretcher clamps can be observed in the corners of the image.

Figure 6.14A shows the thickness change due to stretching force. The change in thickness over force was found to be linear for all experimental results, as the 
simulations predicted. The average experimental thickness was only $0.21 \%$ different than the predicted thickness, with the caveat that there is an outlier value (for Trial 3 , Anterior Surface Up). Thickness was found to change, on average, $-3.90 \%$ for a total force of $0.6 \mathrm{~N}$. The expected change in thickness from simulations was calculated to be $3.70 \%$. The standard deviation of the trials was $0.0298 \mathrm{~mm}$, and $0.0013 \mathrm{~mm}$ for the measurements within the trial.

Figure 6.14B shows the anterior surface (12 $\mathrm{mm}$ surface radius) change due to stretching force. Unlike the prediction, the experimentally measured change in surface radius was not always found to be linear. The average experimental radius was found to be $2.15 \%$ different than the predicted radius. As in the thickness measurements, there is an outlier, in which the overall radii are lower. The standard deviation of the trials was $0.361 \mathrm{~mm}$, and $0.068 \mathrm{~mm}$ for the measurements within the trial. The anterior surface radius was found to change, on average, $-10.86 \%$ for a total force of $0.6 \mathrm{~N}$, compared to the expected change of $-10.15 \%$ from the simulations.

Figure $6.14 \mathrm{C}$ shows the posterior surface $(5 \mathrm{~mm}$ surface radius) change due to stretching force. The change in surface radius was found to be linear across all trials, as the simulations predicted. The average surface radius was found to be $5.84 \%$ different than the predicted values, the highest difference among the measured parameters. The standard deviation of the trials was $0.062 \mathrm{~mm}$, and $0.032 \mathrm{~mm}$ for the measurements within the trial. The posterior surface was found to change, on average, $12.96 \%$, compared to the expected change of $11.24 \%$.

For these key parameters, the behavior observed in the experimental results corresponds to the predictions: a reduction in the AIOL's thickness and flattening of the posterior surface, and a slight curving of the anterior surface.

OCT reconstruction of the lens can be seen in Figure 6.15. Figure 6.15A shows a representative B-Scan from the center of the AIOL and a three-dimensional reconstruction. The stretcher clamp edges can be observed. Figure 6.15B shows a comparison between accommodated and disacommodated states; the change in thickness can be observed. Figure 6.15C shows the AIOL as seen from above.

Figure 6.16 shows a comparison between the AIOL's surfaces reconstructed as a single radius value $(\mathrm{yA}, \mathrm{yP})$ and reconstructed as a conic, with a radius and $\mathrm{k}$ value (yAL, 
yPL). The differences are minor and concentrated on the outermost region of the AIOL's diameter.

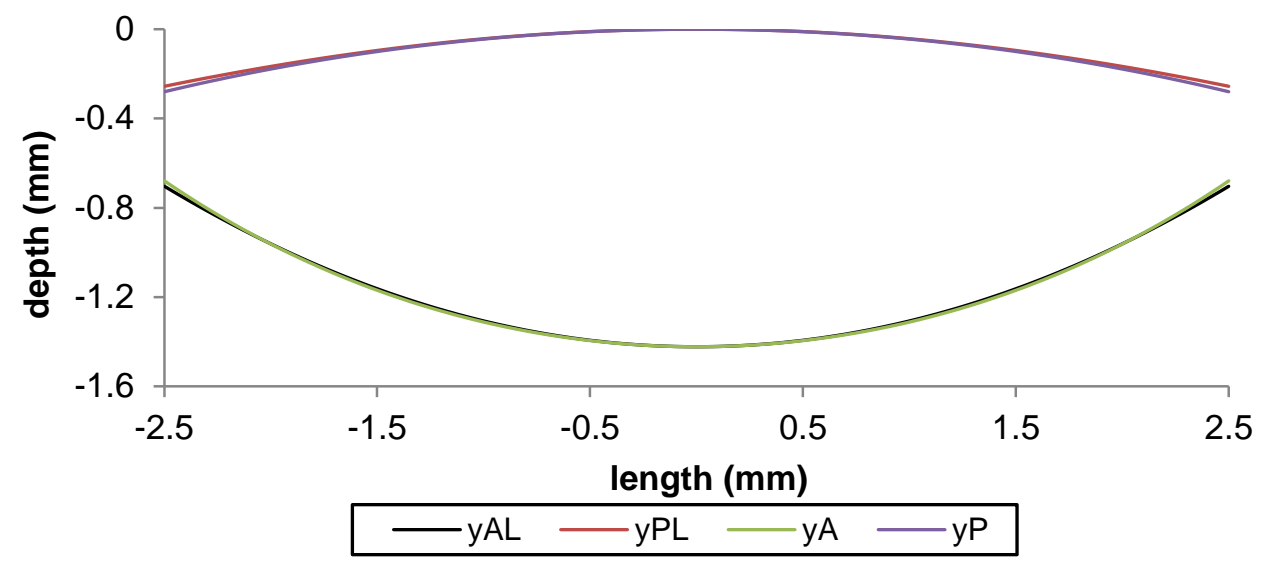

Figure 6.16. Comparison between spherical and conic reconstruction of lens surfaces.

\subsubsection{Comparison between LRT and OCT experiments}

The standard deviation of the LRT results is higher between the cycles of each trial $(9 \%)$ than the standard deviation of the OCT results $(>1 \%)$. The noise in the spot size measurement of the LRT post-processing (which is partly due to image imperfections introduced by the system and partly due to the automated nature of the image processing) is the likely reason for this, with the standard deviation being larger for the initial substeps (where the total magnitude is smaller) than for the subsequent ones. The OCT results are remarkably precise on a per trial basis, suggesting that for further measurements, a briefer measurement protocol could be applied.

In the standard deviation among the trials, however, the OCT system shows larger differences. Each trial represents a distinct experiment; as the AIOL was mounted differently for each trial, some differences could be expected. The standard deviation in the OCT, between trials, is always at least two times larger, and there are some noticeable outlier values in the data. Taking the values obtained as being the true value of the surfaces during that experiment, the expected change in focus can be calculated. Figure 6.17 shows the calculated change in focus for the OCT measurements. The change in focal length for the maximum force applied is consistently higher than the change found in the LRT system, and the deviation between trials is much larger than the one calculated for the LRT system. There is also a small relationship between the 
initial surface radii values and the maximum change in focal length, for the OCT measurements.

This suggests that the variation in surface radii and thickness found in the OCT measurements is an artifact of the experimental set-up and post-processing, rather than an accurate representation of real differences. The posterior surface values obtained in the OCT are more curved than the expected/ nominal value. It is unlikely that the lens can be compressed (due to its haptic geometry bending on compression), suggesting that the OCT system is slightly overstating the curvature.

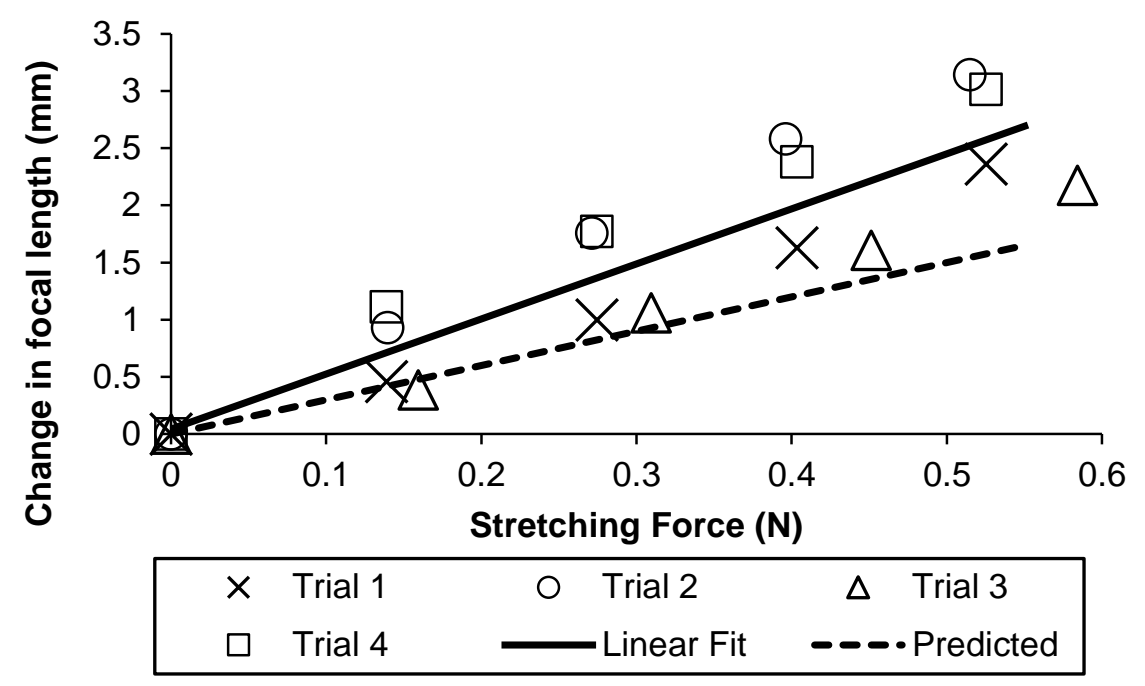

Figure 6.17. Power calculations using OCT surface data.

The results from OCT and LRT system measurements match closely the results predicted by the simulation platform, demonstrating the reliability of the platform. The experimental measurements used a force of $0.55 \mathrm{~N}$, higher than the forces in the accommodative apparatus. The second model was used to evaluate material and design performance in simulated in vivo conditions.

\subsubsection{Evaluation of material parameters in opto-mechanical in vivo model}

Figure 6.18 shows the calculated diopter change for the evaluated materials. The calculated values were found to be low (around $0.4 \mathrm{~m}^{-1}$ ) compared to regulatory minimum values for accommodation $\left(1 \mathrm{~m}^{-1}\right)$ and desired target values $\left(2 \mathrm{~m}^{-1}\right)$. The results were found to be broadly consistent despite the differences in stiffness for all evaluated materials. The main cause for this is the combination of optical and mechanical properties of the evaluated materials. Figure 6.19 shows the relationship 
between the refractive index of the materials and their equivalent Young's modulus. A broadly linear relationship between the properties can be observed. This means the gains obtained from using a material with reduced stiffness were offset by the loss of refractive index, as a lower refractive index requires higher deformation.

This finding suggests that off-the-shelf materials might not yield sufficient change in surface shape and optical power to function as a correction for presbyopia. This is not entirely unsurprising, as hydrophilic polymers are designed for contact lens or intraocular lens applications, and their properties and behavior are typically tailored to these usages. The next step was to use the opto-mechanical model to evaluate for a range of optical and mechanical properties of an idealized material.

Figure 6.20 shows a calculation of the accommodation performance of the finite element model for varying mechanical and optical properties. The Young's Modulus was evaluated at intervals of $0.2 \mathrm{MPa}$ and the refractive index at intervals of 0.01 . The results show that the equivalent Young's Modulus of the material needs to be in the range of 0.1-0.2 $\mathrm{MPa}$, for the finite element model evaluated. The refractive index should be at least 1.42, with better results obtained for higher values of up to 1.46 . The accommodation performance of the model is driven by the mechanical, rather than the optical properties of the material. Although the refractive index is important for accommodation performance, this is only substantially important for low equivalent Young's Moduli. The decrease of accommodative amplitude from 1.46 to 1.40 , is constant for a fixed equivalent Young's Modulus, and is approximately $48 \%$.

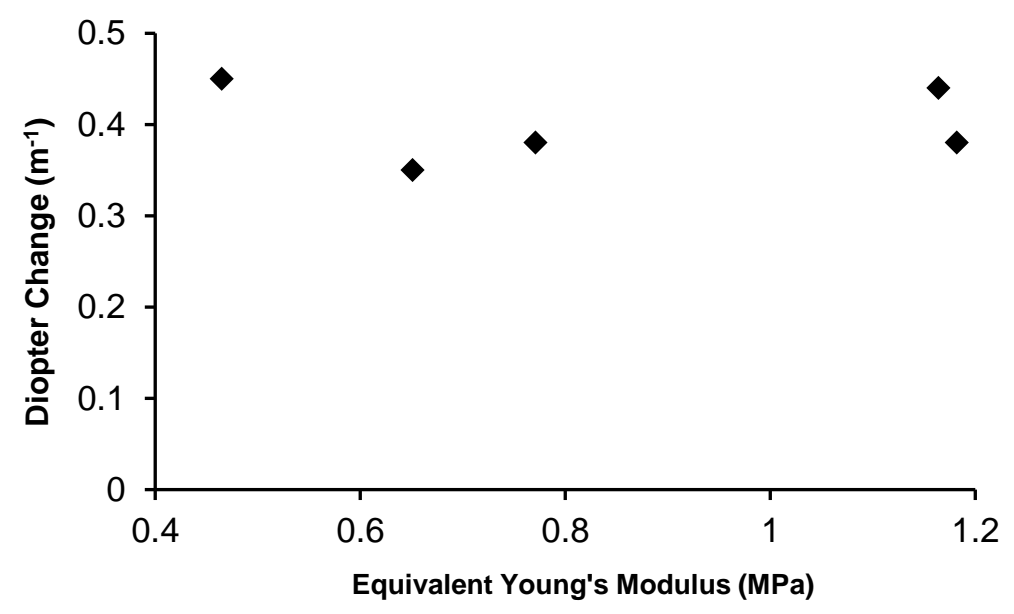

Figure 6.18: Diopter change calculated for all evaluated constitutive models. 


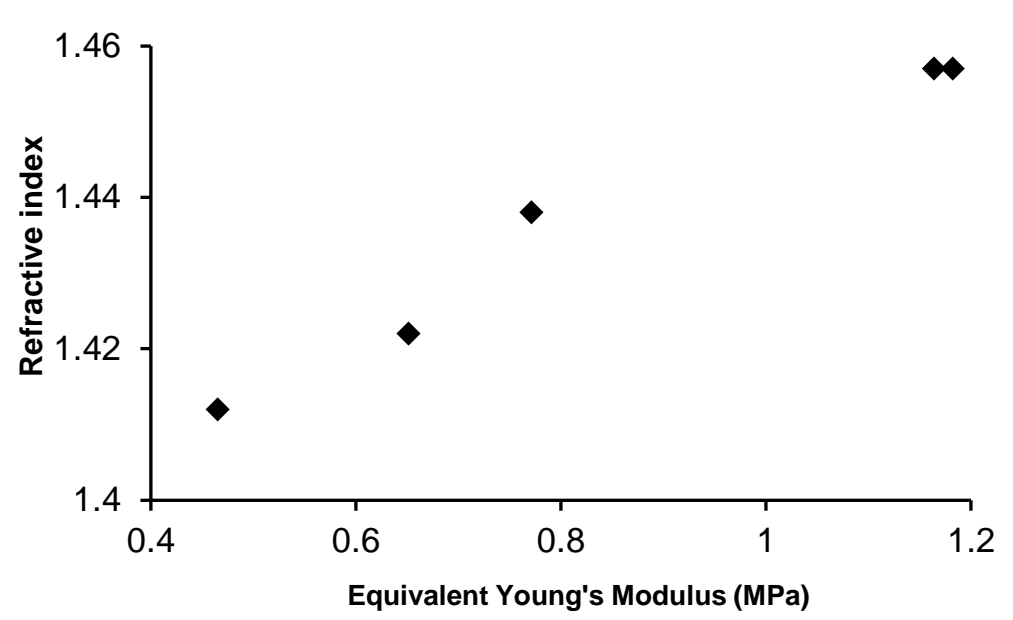

Figure 6.19: Relationship between refractive index and equivalent Young's Modulus.

\subsubsection{Evaluation of geometric parameters in opto-mechanical in vivo model}

Figure 6.21 shows a calculation of the accommodation performance as a function of design parameters. The finite element model was evaluated for varying anterior and posterior radii of curvature. Geometry is found to have some effect on the accommodative amplitude, with a larger posterior radius and a smaller anterior radius increasing the accommodative amplitude. This influence allows for the optimization of the design, but its magnitude is not sufficient to counteract the dominant effect of material stiffness.

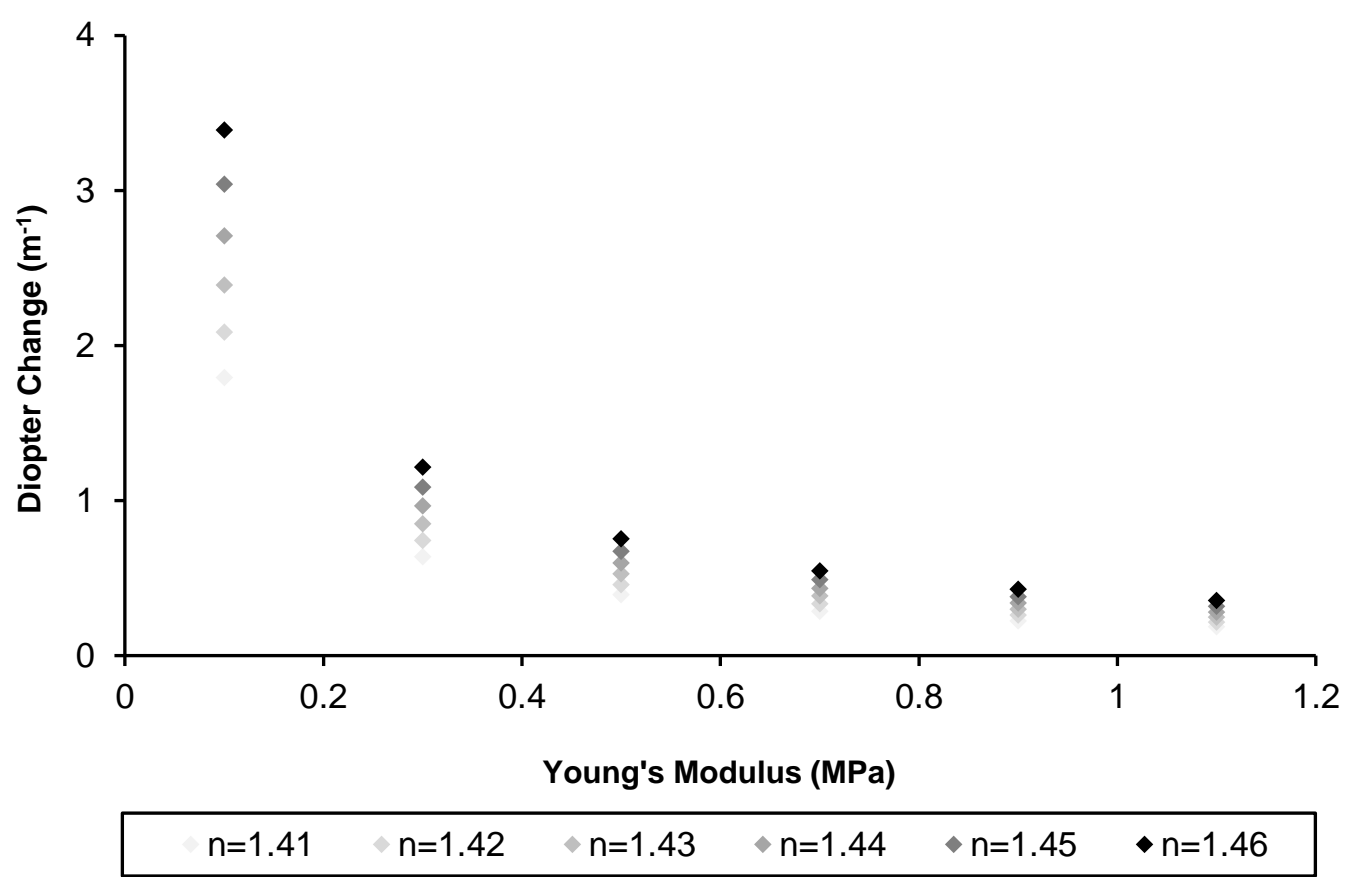

Figure 6.20: Evaluation of accommodative amplitude for varying mechanical and optical properties. 


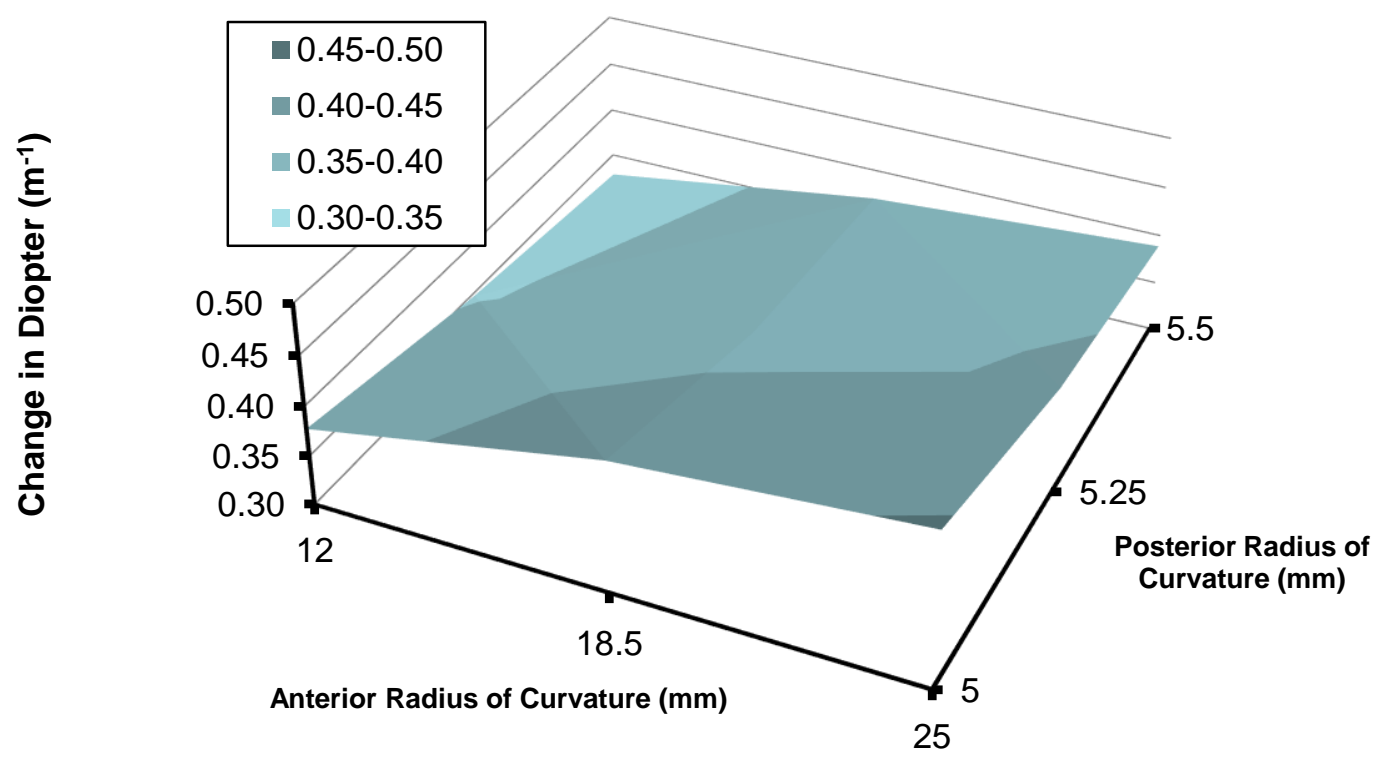

Figure 6.21: Effect of varying anterior and posterior radii of curvature on accommodative amplitude in finite element model.

\subsubsection{AIOL higher order aberrations and their change with accommodation}

Surface topographies were extracted from the finite element simulation and analyzed to investigate potential asymmetries and induction of higher order aberration. The nonzero octo-foil surface terms found in this analysis $(-0.161$ for anterior surface and 0.447 for posterior surface) were consistent with stretching along 8 haptics rather than uniformly along the equator. The effect was minor, with a difference of only $5 \mu \mathrm{m}$ in the periphery of the topographic profile. The measured surface asphericities were $\mathrm{Q}=-0.23$ and -0.62 for the anterior and posterior surfaces, respectively. RMS wave front error was $0.30004 \mu \mathrm{m}$ at 0.1728 of spherical aberration. RMS wavefront error increased to $0.73776 \mu \mathrm{m}$ at maximally accommodated state. The surface maps can be observed in Figure 6.22. 

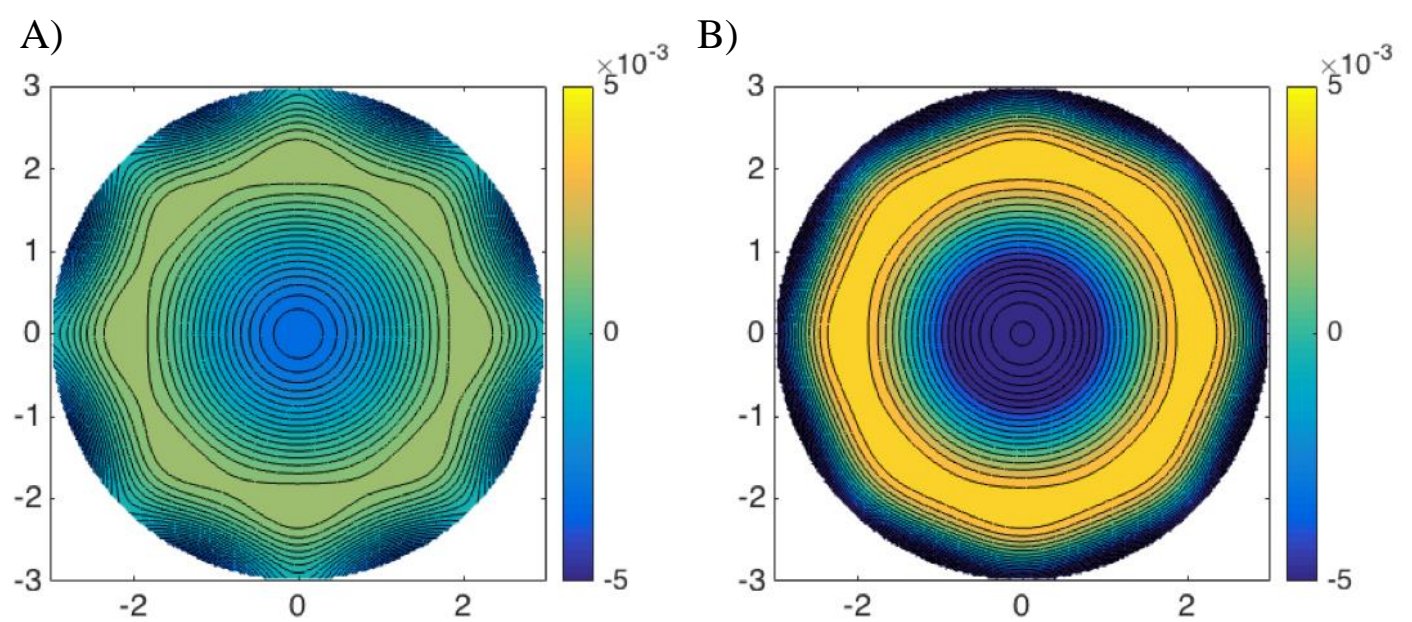

Figure 6.22. Surface maps of disaccommodated AIOL from simulation. A) Anterior surface, B) Posterior surface. Small asymmetry due to loading can be observed.

\subsection{Discussion}

The quantitative techniques (stretcher, LRT, OCT) used in this work show that the AIOL prototype behaves in the predicted way. Its dimensions change in the predicted manner, resulting in a change in power that corresponds to the value predicted from optical simulation. The observations match the behavior of the crystalline lens during accommodation (except the change in anterior surface curvature, likely an effect of bending forces) and show that the proposed design mimics the accommodation process. The simulation platform shows that a surface-changing, accommodating IOL concept can produce a change in surface curvature that can result in a change in optical power. The magnitude of the change in optical power is determined primarily by the mechanical properties of the material, and then by its optical properties.

The forces used in the ex vivo measurements are higher $(0.55 \mathrm{~N})$ than the force found in the accommodative mechanism. The opto-mechanical model was used to calculate the ideal material properties for the proposed AIOL concept. An equivalent Young's Modulus between 0.1-0.2 MPa and a refractive index of 1.43-1.46 are best suited for higher changes in accommodative amplitude (>2D), according to the model. The ranges evaluated for stiffness (0.1-1.1 MPa) and refractive index (1.40-1.46) are within the existing range in standard hydrophilic polymers used in ophthalmology. While Figure 6.19 shows a direct relationship between the equivalent Young's Modulus and the refractive index, the two parameters are not fully dependent. A third parameter, the water content, is directly related to the refractive index and almost entirely determines 
its overall value. Water content is also directly related to stiffness, but is not the only factor affecting it, with chemical composition and cross-linking processes having a large influence, opening the possibility of reducing material stiffness while holding refractive index constant. Therefore, it should be feasible to obtain a material with the desired mechanical properties. The development of such a material falls outside the scope of this work, and is largely an engineering and industrial development rather than a scientific one.

The systems presented are able to quantitative assess the mechanical and optical behavior of an AIOL prototype. The system has some limitations in terms of repeatability and margin of error, as a result of the number of degrees of freedom it requires and the amount of information it is intended to capture. As part of the purpose of this work was to compare experimental results to simulations, measuring the forces required to deform the AIOL was essential. For future work, it should be possible to use the radial stretcher to deform the AIOL without measuring the force. This would allow for a reduction in degrees of freedom on the stretcher, which would make quantification of aberrations and non-symmetrical phenomena viable. The simulation work suggests that asymmetrical effects from the force application are minor (see Figure 6.22). In terms of design input, it is possible that aspheric or conic designs could result in increased accommodation amplitude. To develop the AIOL from concept to fully realized product would require studying these factors.

The prototype and models evaluated in this work represent the simplest version of this AIOL concept: a homogenous material with constant mechanical and optical properties. The real crystalline lens has both mechanical and optical heterogeneity, with distinct regions that respond differently under loading, affecting the accommodative amplitude. Furthermore, the refractive index is spatially varying as well, affecting the overall lens power. It is possible, then, that processes by which mechanical and optical properties of the polymer can be spatially varied could reduce requirements for the ideal AIOL polymer.

Further work is necessary to develop a fully working AIOL. For the prototype presented and evaluated, the haptics were manufactured as flat. A final version of haptic geometry must be curved, and with a transversal height of at least $1 \mathrm{~mm}$, allowing for full engagement with the equatorial capsular region and offering separation of the capsular 
bag. An ideal experiment would be to implant the lens ex vivo, and mount the physiological structure (ciliary body, zonules, lens capsule, and AIOL) onto the radial stretcher, for evaluation with LRT and OCT systems. In practice, the fragile nature of these tissues might make such mounting, and the subsequent loading, impractical. Current approaches for photobonding have involved directly applying the Rose Bengal onto the entire surface by soaking the object in a Rose Bengal solution. The absorption of light by Rose Bengal covering all surfaces can reduce the irradiation reaching the external surface of the haptic. AIOL implantation would require the use of a controlled quantity of Rose Bengal to release during the implantation process, minimizing toxicity concerns while maximizing bonding strength. Proposals include encapsulating the Rose Bengal in nanoparticles, delivering it through a microfluidic channel or structural deposit, or a controlled and localized soaking procedure. The mechanical and optical interaction, if any, between the deformable optic and the second optic is another aspect requiring further development and study.

\subsection{Conclusion}

A surface-changing AIOL prototype was evaluated mechanically and optically and shown to change the shape of its surfaces and its thickness, with a corresponding change in optical power, matching the predictions made by our simulation platform. Future work will focus on material tuning, haptic design, and implantation. 


\section{Conclusions}

In this thesis,

- Inverse modeling procedures for retrieving mechanical properties from the cornea were developed using optical imaging, air puff deformation, and finite element software

- The use of an optical technology to bond biocompatible silk fibroin films to the cornea was demonstrated for treatment of wounds or grafts.

- Inverse modeling procedures for retrieving mechanical properties from the crystalline lens using Brillouin optical microscopy and mechanical testing, and a finite element model were developed to validate the Brillouin models.

- A novel accommodating lens concept for presbyopia was developed, along with opto-mechanical models to assess its viability and necessary parameters.

- An accommodation system with mechanical and optical measurement and quantification systems was implemented.

- An accommodating lens prototype was fabricated and evaluated ex vivo.

And our findings were:

- The isotropic mechanical properties of model and porcine corneas can be reconstructed from air puff tonometry data, accounting for the effect of geometry.

- Silk fibroin can be successfully bonded to the cornea using green light and Rose Bengal, and the bonding strength can be controlled by the irradiation dose.

- The spatially varying mechanical properties of the crystalline lens can be reconstructed through Brillouin optical microscopy and inverse modeling. However, the reconstructed elasticity profile yields a limited power change in a finite element model of accommodation, suggesting that the Brillouin optical microscopy data is not sufficient to describe the lens' behavior under loading.

- The behavior of a surface-changing accommodating lens can be predicted and accurately evaluated with an opto-mechanical model, as well as on an accommodation system. 
- Such a lens could yield power change to correct for presbyopia with material properties within the range of currently existing commercial materials.

A number of scientific and industrial advances should continue the work presented in this thesis.

The inverse modeling procedure for the retrieval of corneal mechanical properties can be expanded by including additional relevant unknowns, the most important being the intraocular pressure.

The biocompatibility and the function of the silk fibroin films should be evaluated in cell cultures and in vivo, in order to assess their use in corneal wound healing. The mechanical, optical, and topographic parameters of the silk fibroin films can then be tailored to their function.

Additional measurements of Brillouin optical microscopy, with corresponding compression measurements, should allow for a more thorough correlation between both measurements. Another form of mechanical testing, such as loading under an accommodation system as described in this work, should allow for more accurate targets to assess the correlation between the Brillouin elasticity models and the lens' behavior.

Work should be undertaken to develop a hydrophilic polymer with parameters that match the targets described in this work. Additionally, more sophisticated optical models can be developed to more thoroughly assess an AIOL's optical performance. With these advances, a prototype that more closely resembles the desired performance can be evaluated in the accommodation platform. Furthermore, a full prototype could be photobonded to the capsular bag, and the entire structure could be evaluated in the accommodation platform. 


\section{List of Disseminations}

\section{Papers}

- de la Hoz A., Germann J., Martinez-Enriquez E., Pascual D., Bekesi N., Alejandre-Alba N., Dorronsoro C., Marcos S. (2019) "Design and ex situ performance of a shape-changing accommodating intraocular lens." Optica 6(8), 1050-1057.

- Bekesi N., Dorronsoro C., de la Hoz A., Marcos S. (2016) "Material Properties from Air Puff Corneal Deformation by Numerical Simulations on Model Corneas" PLOS ONE 11(10), e0165669.

\section{Conference papers}

- de la Hoz A., Enriquez-Martinez E., Subhash H., Pascual D., Bekesi N., Dorronsoro C., Alejandre-Alba N., Marcos S. (2016) Design and evaluation of a surface-changing accommodating IOL. ARVO Annual Meeting, Program number 3131-B0136.

- de la Hoz A., Enriquez-Martinez E., Subhash H., Pascual D., Bekesi N., Dorronsoro C., Alejandre-Alba N., Marcos S. (2016) Surface-changing Accommodating Intraocular Lens for Presbyopia Correction. Frontiers in Optics.

- de la Hoz A., Enriquez-Martinez E., Germann, J., Pascual D., Bekesi N., Dorronsoro C., Alejandre-Alba N., Marcos S. (2018) Diseño y Evaluación de una nueva Lente Acomodativa Deformable. Reunión Nacional de Óptica.

- de la Hoz, A., Kochevar I., Omenetto F., Marcos S. (2019) Photobonded silkfibroin films for corneal dressing. ARVO Annual Meeting. Program number 3146249.

- de la Hoz A., Scarcelli G., Yun, S.H., Marcos S. (2017) Finite Element Accommodation models using elasticity gradients from Brillouin microscopy. ARVO Annual Meeting, program number 326-B0571. 



\section{Bibliography}

Adhi, M., \& Duker, J. S. (2013). Optical coherence tomography--current and future applications. Current opinion in ophthalmology, 24(3), 213-221.

Agarwal, A., Kumar, D. A., Jacob, S., Baid, C., Agarwal, A., \& Srinivasan, S. (2008). Fibrin glue assisted sutureless posterior chamber intraocular lens implantation in eyes with deficient posterior capsules. Journal of Cataract \& Refractive Surgery, 34(9), 1433-1438.

Alejandre-Alba, N., Gutierrez-Contreras, R., Dorronsoro, C., \& Marcos, S. (2018). Intraocular Photobonding to Enable Accommodating Intraocular Lens Function. Translational vision science \& technology, 7(5), 27-27.

Ali, A., Fouladi, M. H., \& Sahari, B. (2010). A review of constitutive models for rubber-like materials. American Journal of Engineering and Applied Sciences, 3(1), 232-239.

Alió, J. L., Alió Del Barrio, J. L., \& Vega-Estrada, A. (2017). Accommodative intraocular lenses: where are we and where we are going. Eye and vision (London, England), 4, 16-16.

Alió, J. L., Ben-nun, J., Rodríguez-Prats, J. L., \& Plaza, A. B. (2009). Visual and accommodative outcomes 1 year after implantation of an accommodating intraocular lens based on a new concept. Journal of Cataract \& Refractive Surgery, 35(10), 1671-1678.

Alio, J. L., Plaza-Puche, A. B., Fernandez-Buenaga, R., Pikkel, J., \& Maldonado, M. (2017). Multifocal intraocular lenses: an overview. Survey of Ophthalmology, 62(5), 611-634.

Alió, J. L., Plaza-Puche, A. B., Montalban, R., \& Ortega, P. (2012). Near visual outcomes with single-optic and dual-optic accommodating intraocular lenses. Journal of Cataract \& Refractive Surgery, 38(9), 1568-1575.

Alió, J. L., Simonov, A., Romero, D., Angelov, A., Angelov, Y., van Lawick, W., \& Rombach, M. (2018). Analysis of Accommodative Performance of a New Accommodative Intraocular Lens. Journal of Refractive Surgery, 34, 78-83.

Allen, C. L., Clare, G., Stewart, E. A., Branch, M. J., McIntosh, O. D., Dadhwal, M., . . - Hopkinson, A. (2013). Augmented dried versus cryopreserved amniotic membrane as an ocular surface dressing. PLOS ONE, 8(10), e78441-e78441.

Altman, G. H., Diaz, F., Jakuba, C., Calabro, T., Horan, R. L., Chen, J., . . Kaplan, D. L. (2003). Silk-based biomaterials. Biomaterials, 24(3), 401-416.

Ang, R., Martinez, G., Cruz, E., Tiongson, A., \& Dela Cruz, A. (2013). Prospective evaluation of visual outcomes with three presbyopia-correcting intraocular lenses following cataract surgery. Clinical ophthalmology (Auckland, N.Z.), 7, 1811-1823.

Athanasiou, K. A., \& Natoli, R. M. (2008). Introduction to Continuum Biomechanics. Synthesis Lectures on Biomedical Engineering, 3(1), 1-206.

Bailey, S. T., Twa, M. D., Gump, J. C., Venkiteshwar, M., Bullimore, M. A., \& Sooryakumar, R. (2010). Light-scattering study of the normal human eye lens: elastic properties and age dependence. IEEE transactions on bio-medical engineering, 57(12), 2910-2917. 
Bassnett, S., Shi, Y., \& Vrensen, G. F. J. M. (2011). Biological glass: structural determinants of eye lens transparency. Philosophical transactions of the Royal Society of London. Series B, Biological sciences, 366(1568), 1250-1264.

Beer, P. (2015). US Patent No. 9220590B2.

Besner, S., Scarcelli, G., Pineda, R., \& Yun, S.-H. (2016). In Vivo Brillouin Analysis of the Aging Crystalline Lens. Investigative Ophthalmology \& Visual Science, 57(13), 5093-5100.

Bhardwaj, V., \& Rajeshbhai, G. P. (2013). Axial length, anterior chamber depth-a study in different age groups and refractive errors. Journal of clinical and diagnostic research : JCDR, 7(10), 2211-2212.

Birkenfeld, J., de Castro, A., Ortiz, S., Pascual, D., \& Marcos, S. (2013). Contribution of the gradient refractive index and shape to the crystalline lens spherical aberration and astigmatism. Vision Research, 86, 27-34.

Burd, H. J., Judge, S. J., \& Cross, J. A. (2002). Numerical modelling of the accommodating lens. Vision Research, 42(18), 2235-2251.

Charman, W. N. (2008). The eye in focus: accommodation and presbyopia. Clinical and Experimental Optometry, 91(3), 207-225.

Charman, W. N., \& Atchison, D. A. (2014). Age-dependence of the average and equivalent refractive indices of the crystalline lens. Biomedical Optics Express, 5(1), 31-39.

Cleary, G., Spalton, D. J., \& Gala, K. B. (2010). A Randomized Intraindividual Comparison of the Accommodative Performance of the Bag-in-the-Lens Intraocular Lens in Presbyopic Eyes. American Journal of Ophthalmology, 150(5), 619-627.e611.

Croft, M. A., McDonald, J. P., Katz, A., Lin, T.-L., Lütjen-Drecoll, E., \& Kaufman, P. L. (2013). Extralenticular and Lenticular Aspects of Accommodation and Presbyopia in Human Versus Monkey EyesZonula and Ciliary Muscle Function. Investigative Ophthalmology \& Visual Science, 54(7), 5035-5048.

Crouch, J. R., Merriam, J. C., Iii, E. R. C., \& Khandji, A. (2004). Finite Element Analysis Applied to Cornea Modeling and Tissue Deformation. Investigative Ophthalmology \& Visual Science, 45(13), 309-309.

Day, J., \& Miller, K. (2000, 2000). Equibiaxial Stretching of Elastomeric Sheets, An Analytical Verification of Experimental Technique. Paper presented at the ABAQUS users' conference, Newport, RI.

de Castro, A., Birkenfeld, J., Heilman, B. M., Ruggeri, M., Arrieta, E., Parel, J.-M., . . . Marcos, S. (2019). Off-axis optical coherence tomography imaging of the crystalline lens to reconstruct the gradient refractive index using optical methods. Biomedical Optics Express, 10(7), 3622-3634.

de Castro, A., Ortiz, S., Gambra, E., Siedlecki, D., \& Marcos, S. (2010). Threedimensional reconstruction of the crystalline lens gradient index distribution from OCT imaging. Optics Express, 18(21), 21905-21917.

de Castro, A., Siedlecki, D., Borja, D., Uhlhorn, S., Parel, J.-M., Manns, F., \& Marcos, S. (2011). Age-dependent variation of the Gradient Index profile in human crystalline lenses. Journal of modern optics, 58(19-20), 1781-1787.

Dennis, B. H., Jin, W., Dulikravich, G., \& Jaric, J. (2011). Application of the finite element method to inverse problems in solid mechanics. Journal of Structural Changes in Solids, 3, 11-21.

Dong, J., Zhang, Y., Zhang, H., Jia, Z., Zhang, S., \& Wang, X. (2018). Comparison of axial length, anterior chamber depth and intraocular lens power between 
IOLMaster and ultrasound in normal, long and short eyes. PLOS ONE, 13(3), $\mathrm{e} 0194273$.

Dorronsoro, C., Alejandre-Alba, N., Bekesi, N., \& Marcos, S. (2017). ES EP WO US Patent No.

Dorronsoro, C., Pascual, D., Pérez-Merino, P., Kling, S., \& Marcos, S. (2012). Dynamic OCT measurement of corneal deformation by an air puff in normal and crosslinked corneas. Biomedical Optics Express, 3(3), 473-487.

Doyle, T. C., \& Ericksen, J. L. (1956). Nonlinear Elasticity. In H. L. Dryden \& T. von Kármán (Eds.), Advances in Applied Mechanics (Vol. 4, pp. 53-115): Elsevier.

Dua, H. S., Gomes, J. A. P., King, A. J., \& Maharajan, V. S. (2004). The amniotic membrane in ophthalmology. Survey of Ophthalmology, 49(1), 51-77.

Dubbelman, M., Van der Heijde, G. L., Weeber, H. A., \& Vrensen, G. F. J. M. (2003). Changes in the internal structure of the human crystalline lens with age and accommodation. Vision Research, 43(22), 2363-2375.

Ehrmann, K., Ho, A., \& Parel, J.-M. (2004). Ex vivo accommodation simulator II: concept and preliminary results. Paper presented at the Biomedical Optics 2004.

Elsheikh, A., Wang, D., \& Pye, D. (2007). Determination of the Modulus of Elasticity of the Human Cornea. Journal of refractive surgery (Thorofare, N.J. : 1995), 23, 808-818.

Fagerholm, P. P., Philipson, B. T., \& Lindström, B. (1981). Normal human lens-the distribution of protein. Experimental Eye Research, 33(6), 615-620.

Fercher, A. F. (2010). Optical coherence tomography - development, principles, applications. Zeitschrift für Medizinische Physik, 20(4), 251-276.

Fisher, R. F. (1971). The elastic constants of the human lens. The Journal of Physiology, 212, 147-180.

Fisher, R. F. (1977). The force of contraction of the human ciliary muscle during accommodation. The Journal of Physiology, 270(1), 51-74.

Fratzl, P., Misof, K., Zizak, I., Rapp, G., Amenitsch, H., \& Bernstorff, S. (1998). Fibrillar Structure and Mechanical Properties of Collagen. Journal of Structural Biology, 122(1), 119-122.

Gambra, E. (2015). Accommodation: Optical Function and Crystalline Lens Imaging. (Doctorate), Universidad de Valladolid, Valladolid, Spain.

Gambra, E., Ortiz, S., Perez-Merino, P., Gora, M., Wojtkowski, M., \& Marcos, S. (2013). Static and dynamic crystalline lens accommodation evaluated using quantitative 3-D OCT. Biomedical Optics Express, 4(9), 1595-1609.

Gicquel, J.-J., Dua, H. S., Brodie, A., Mohammed, I., Suleman, H., Lazutina, E., . . . Hopkinson, A. (2009). Epidermal Growth Factor Variations in Amniotic Membrane Used for Ex Vivo Tissue Constructs. Tissue Engineering Part A, 15(8), 1919-1927.

Glass, D. H., Roberts, C. J., Litsky, A. S., \& Weber, P. A. (2008). A Viscoelastic Biomechanical Model of the Cornea Describing the Effect of Viscosity and Elasticity on Hysteresis. Investigative Ophthalmology \& Visual Science, 49(9), 3919-3926.

Glasser, A., \& Campbell, M. C. W. (1998). Presbyopia and the optical changes in the human crystalline lens with age. Vision Research, 38(2), 209-229.

Glasser, A., \& Wendt, M. (2013). Age-related loss of accommodation in rhesus monkeys is associated with an age-related increase in lens stiffness. Investigative Ophthalmology \& Visual Science, 54, 4274. 
Harkin, D. G., George, K. A., Madden, P. W., Schwab, I. R., Hutmacher, D. W., \& Chirila, T. V. (2011). Silk fibroin in ocular tissue reconstruction. Biomaterials, 32(10), 2445-2458.

Hermans, E. A., Dubbelman, M., van der Heijde, G. L., \& Heethaar, R. M. (2006). Estimating the external force acting on the human eye lens during accommodation by finite element modelling. Vision Research, 46(21), 36423650 .

Hernandez, V. M., Cabot, F., Ruggeri, M., de Freitas, C., Ho, A., Yoo, S., . . Manns, F. (2015). Calculation of crystalline lens power using a modification of the Bennett method. Biomedical Optics Express, 6(11), 4501-4515.

Hollister, S. J. BME 456: Biosolid Mechanics: Modeling and Applications. Retrieved from http://www.umich.edu/ bme456/

Jones, C. E., Atchison, D. A., Meder, R., \& Pope, J. M. (2005). Refractive index distribution and optical properties of the isolated human lens measured using magnetic resonance imaging (MRI). Vision Research, 45(18), 2352-2366.

Kaji, Y., Usui, T., Oshika, T., Matsubara, M., Yamashita, H., Araie, M., . . Amano, S. (2000). Advanced Glycation End Products in Diabetic Corneas. Investigative Ophthalmology \& Visual Science, 41(2), 362-368.

Kasthurirangan, S., Markwell, E. L., Atchison, D. A., \& Pope, J. M. (2011). MRI study of the changes in crystalline lens shape with accommodation and aging in humans. Journal of Vision, 11(3), 19-19.

Kim, H.-S., Yu, D.-S., Cho, H. G., Moon, B.-Y., \& Kim, S.-Y. (2019). Comparison of predicted and measured axial length for ophthalmic lens design. PLOS ONE, 14(1), e0210387.

Kling, S., Bekesi, N., Dorronsoro, C., Pascual, D., \& Marcos, S. (2014). Corneal Viscoelastic Properties from Finite-Element Analysis of In Vivo Air-Puff Deformation. PLOS ONE, 9(8), e104904.

Koeppl, C., Findl, O., Menapace, R., Kriechbaum, K., Wirtitsch, M., Buehl, W., ... Drexler, W. (2005). Pilocarpine-induced shift of an accommodating intraocular lens: AT-45 Crystalens. Journal of Cataract \& Refractive Surgery, 31(7), 12901297.

Kohl, J. C., Werner, L., Ford, J. R., Cole, S. C., Vasavada, S. A., Gardiner, G. L., . . . Mamalis, N. (2014). Long-term uveal and capsular biocompatibility of a new accommodating intraocular lens. Journal of Cataract \& Refractive Surgery, 40(12), 2113-2119.

Koopmans, S. A., Terwee, T., Glasser, A., Wendt, M., Vilupuru, A. S., van Kooten, T. G., . . . Kooijman, A. C. (2006). Accommodative lens refilling in rhesus monkeys. Investigative Ophthalmology \& Visual Science, 47(7), 2976-2984.

Koski, K. J., Akhenblit, P., McKiernan, K., \& Yarger, J. L. (2013). Non-invasive determination of the complete elastic moduli of spider silks. Nature Materials, $12,262$.

Krag, S., Olsen, T., \& Andreassen, T. T. (1997). Biomechanical characteristics of the human anterior lens capsule in relation to age. Investigative Ophthalmology \& Visual Science, 38(2), 357-363.

Kuwabara, T. (1975). The maturation of the lens cell: a morphologic study. Experimental Eye Research, 20(5), 427-443.

Lan, J., Huang, Y.-S., Dai, Y.-H., Wu, X.-M., Sun, J.-J., \& Xie, L.-X. (2017). Visual performance with accommodating and multifocal intraocular lenses. International journal of ophthalmology, 10(2), 235-240. 
Lanchares, E., Navarro, R., \& Calvo, B. (2012). Hyperelastic modelling of the crystalline lens: Accommodation and presbyopia. Journal of Optometry, 5(3), 110-120.

Langenbucher, A., Huber, S., Nguyen, N. X., Seitz, B., Gusek-Schneider, G. C., \& Küchle, M. (2003). Measurement of accommodation after implantation of an accommodating posterior chamber intraocular lens11None of the authors has a financial or proprietary interest in any material or method mentioned. Journal of Cataract \& Refractive Surgery, 29(4), 677-685.

Lawrence, B. D., Marchant, J. K., Pindrus, M. A., Omenetto, F. G., \& Kaplan, D. L. (2009). Silk film biomaterials for cornea tissue engineering. Biomaterials, 30(7), 1299-1308.

Legeais, J.-M., Werner, L., Werner, L., Abenhaim, A., \& Renard, G. (1999). Pseudoaccommodation: BioComFoldversus a foldable silicone intraocular lens. Journal of Cataract \& Refractive Surgery, 25(2), 262-267.

Levin, L., Nilsson, S., Ver Hoeve, J., Wu, S., Kaufman, P. L., \& Alm, A. (2011). Adler's Physiology of the Eye (11 ed.): Saunders.

Lim, J. C., Walker, K. L., Sherwin, T., Schey, K. L., \& Donaldson, P. J. (2009). Confocal Microscopy Reveals Zones of Membrane Remodeling in the Outer Cortex of the Human Lens. Investigative Ophthalmology \& Visual Science, 50(9), 4304-4310.

Liu, T.-1., Miao, J.-c., Sheng, W.-h., Xie, Y.-f., Huang, Q., Shan, Y.-b., \& Yang, J.-c. (2010). Cytocompatibility of regenerated silk fibroin film: a medical biomaterial applicable to wound healing. Journal of Zhejiang University. Science. B, 11(1), 10-16.

Liu, Z., Wang, B., Xu, X., \& Wang, C. (2006). A study for accommodating the human crystalline lens by finite element simulation. Computerized Medical Imaging and Graphics, 30(6), 371-376.

Ljubimova, D., Eriksson, A., \& Bauer, S. (2008). Aspects of eye accommodation evaluated by finite elements. Biomechanics and Modeling in Mechanobiology, $7(2), 139-150$.

Loewenstein, M. A., \& Bettelheim, F. A. (1976). Comparative rheology of cornea. Comparative Biochemistry and Physiology Part A: Physiology, 55(4), 363-365.

Lu, S., Wang, X., Lu, Q., Zhang, X., Kluge, J. A., Uppal, N., . . Kaplan, D. L. (2010). Insoluble and Flexible Silk Films Containing Glycerol. Biomacromolecules, 11(1), 143-150.

Manns, F., Parel, J.-M., Denham, D., Billotte, C., Ziebarth, N., Borja, D., . . Holden, B. (2007). Optomechanical Response of Human and Monkey Lenses in a Lens Stretcher. Investigative Ophthalmology \& Visual Science, 48(7), 3260-3268.

Marcos, S., Alejandre, N., Lamela, J., Dorronsoro, C., \& Kochevar, I. E. (2015). Toward New Engagement Paradigms For Intraocular Lenses: Light-Initiated Bonding of Capsular Bag to Lens Materials. Investigative Ophthalmology \& Visual Science, 56(8), 4249-4256.

Marcos, S., Ortiz, S., Pérez-Merino, P., Birkenfeld, J., Durán, S., \& Jiménez-Alfaro, I. (2014). Three-Dimensional Evaluation of Accommodating Intraocular Lens Shift and Alignment In Vivo. Ophthalmology, 121(1), 45-55.

Marques, E. F., \& Castanheira-Dinis, A. (2014). Clinical performance of a new aspheric dual-optic accommodating intraocular lens. Clinical ophthalmology (Auckland, N.Z.), 8, 2289-2295. 
Martinez-Enriquez, E., Pérez-Merino, P., Velasco-Ocana, M., \& Marcos, S. (2017). OCT-based full crystalline lens shape change during accommodation in vivo. Biomedical Optics Express, 8(2), 918-933.

Martinez-Enriquez, E., Sun, M., Velasco-Ocana, M., Birkenfeld, J., Pérez-Merino, P., \& Marcos, S. (2016). Optical Coherence Tomography Based Estimates of Crystalline Lens Volume, Equatorial Diameter, and Plane PositionOCT-Based Estimation of the Entire Crystalline Lens Geometry. Investigative Ophthalmology \& Visual Science, 57(9), OCT600-OCT610.

Mastropasqua, L., Toto, L., Falconio, G., Nubile, M., Carpineto, P., Ciancaglini, M., . . . Ballone, E. (2007). Longterm results of 1 CUß accommodative intraocular lens implantation: 2-year follow-up study. Acta Ophthalmologica Scandinavica, 85(4), 409-414.

McFall-Ngai, M. J., Ding, L. L., Takemoto, L. J., \& Horwitz, J. (1985). Spatial and temporal mapping of the age-related changes in human lens crystallins. Experimental Eye Research, 41(6), 745-758.

McLeod, S. D. (2006). Optical principles, biomechanics, and initial clinical performance of a dual-optic accommodating intraocular lens (an American Ophthalmological Society thesis). Transactions of the American Ophthalmological Society, 104, 437-452.

Michael, R., \& Bron, A. J. (2011). The ageing lens and cataract: a model of normal and pathological ageing. Philosophical transactions of the Royal Society of London. Series B, Biological sciences, 366(1568), 1278-1292.

Mitsuhashi, K., Ghosh, S., \& Koibuchi, H. (2018). Mathematical Modeling and Simulations for Large-Strain J-Shaped Diagrams of Soft Biological Materials. Polymers, 10(7), 715.

Musgrave, C. S. A., \& Fang, F. (2019). Contact Lens Materials: A Materials Science Perspective. Materials (Basel, Switzerland), 12(2), 261.

Nejad, T. M., Foster, C., \& Gongal, D. (2014). Finite element modelling of cornea mechanics: a review. Arquivos Brasileiros de Oftalmologia, 77, 60-65.

Nguyen, T.-M., Aubry, J.-F., Fink, M., Bercoff, J., \& Tanter, M. (2014). In Vivo Evidence of Porcine Cornea Anisotropy Using Supersonic Shear Wave Imaging. Investigative Ophthalmology \& Visual Science, 55(11), 7545-7552.

Nguyen, T. D., \& Boyce, B. L. (2011). An inverse finite element method for determining the anisotropic properties of the cornea. Biomechanics and Modeling in Mechanobiology, 10(3), 323-337.

Nibourg, L. M., Sharma, P. K., van Kooten, T. G., \& Koopmans, S. A. (2015). Changes in lens stiffness due to capsular opacification in accommodative lens refilling. Experimental Eye Research, 134, 148-154.

Nishi, O., Nishi, K., Nishi, Y., \& Chang, S. (2008). Capsular bag refilling using a new accommodating intraocular lens. Journal of cataract and refractive surgery, 34(2), 302-309.

Ortiz, S. (2012). Quantitative Anterior Segment Optical Coherence Tomography. (Doctorate Thesis), Universidad de Valladolid, Valladolid, Spain.

Ortiz, S., Pérez-Merino, P., Durán, S., Velasco-Ocana, M., Birkenfeld, J., de Castro, A., . . . Marcos, S. (2013). Full OCT anterior segment biometry: an application in cataract surgery. Biomedical Optics Express, 4(3), 387-396.

Ortiz, S., Siedlecki, D., Grulkowski, I., Remon, L., Pascual, D., Wojtkowski, M., \& Marcos, S. (2010). Optical distortion correction in Optical Coherence Tomography for quantitative ocular anterior segment by three-dimensional imaging. Optics Express, 18(3), 2782-2796. 
Ortiz, S., Siedlecki, D., Pérez-Merino, P., Chia, N., de Castro, A., Szkulmowski, M., .. . Marcos, S. (2011). Corneal topography from spectral optical coherence tomography (sOCT). Biomedical Optics Express, 2(12), 3232-3247.

Ortiz, S., Siedlecki, D., Remon, L., \& Marcos, S. (2009). Optical coherence tomography for quantitative surface topography. Applied Optics, 48(35), 6708-6715.

Panilaitis, B., Altman, G. H., Chen, J., Jin, H.-J., Karageorgiou, V., \& Kaplan, D. L. (2003). Macrophage responses to silk. Biomaterials, 24(18), 3079-3085.

Pardue, M. T., \& Sivak, J. G. (2000). Age-Related Changes in Human Ciliary Muscle. Optometry and Vision Science, 77(4), 204-210.

Peng, Q., Yang, Y., \& Zhang, X. (2003). US Patent No.

Pérez-Merino, P., Birkenfeld, J., Dorronsoro, C., Ortiz, S., Durán, S., Jiménez-Alfaro, I., \& Marcos, S. (2014). Aberrometry in Patients Implanted With Accommodative Intraocular Lenses. American Journal of Ophthalmology, 157(5), 1077-1089.e1071.

Pérez-Merino, P., Ortiz, S., Alejandre, N., Jiménez-Alfaro, I., \& Marcos, S. (2013). Quantitative OCT-Based Longitudinal Evaluation of Intracorneal Ring Segment Implantation in Keratoconus. Investigative Ophthalmology \& Visual Science, 54(9), 6040-6051.

Pérez-Merino, P., Velasco-Ocana, M., Martinez-Enriquez, E., \& Marcos, S. (2015). OCT-based crystalline lens topography in accommodating eyes. Biomedical Optics Express, 6(12), 5039-5054.

Qazi, Y., Wong, G., Monson, B., Stringham, J., \& Ambati, B. K. (2010). Corneal transparency: genesis, maintenance and dysfunction. Brain research bulletin, 81(2-3), 198-210.

Rao, P. V., Huang, Q.-1., Horwitz, J., \& Zigler, J. S. (1995). Evidence that $\alpha$-crystallin prevents non-specific protein aggregation in the intact eye lens. Biochimica et Biophysica Acta (BBA) - General Subjects, 1245(3), 439-447.

Reilly, M. A., \& Cleaver, A. (2017). Inverse elastographic method for analyzing the ocular lens compression test. Journal of Innovative Optical Health Sciences, 10(06), 1742009.

Reiß, S., Burau, G., Stachs, O., Guthoff, R., \& Stolz, H. (2011). Spatially resolved Brillouin spectroscopy to determine the rheological properties of the eye lens. Biomedical Optics Express, 2(8), 2144-2159.

Richdale, K., Bullimore, M. A., \& Zadnik, K. (2008). Lens thickness with age and accommodation by optical coherence tomography. Ophthalmic \& physiological optics : the journal of the British College of Ophthalmic Opticians (Optometrists), 28(5), 441-447.

Rockwood, D. N., Preda, R. C., Yücel, T., Wang, X., Lovett, M. L., \& Kaplan, D. L. (2011). Materials fabrication from Bombyx mori silk fibroin. Nature Protocols, $6,1612$.

Römer, L., \& Scheibel, T. (2008). The elaborate structure of spider silk: structure and function of a natural high performance fiber. Prion, 2(4), 154-161.

Rosen, A. M., Denham, D. B., Fernandez, V., Borja, D., Ho, A., Manns, F., . . . Augusteyn, R. C. (2006). In vitro dimensions and curvatures of human lenses. Vision Research, 46(6), 1002-1009.

Salerno, L. C., Tiveron, M. C., Jr., \& Alió, J. L. (2017). Multifocal intraocular lenses: Types, outcomes, complications and how to solve them. Taiwan journal of ophthalmology, 7(4), 179-184. 
Santhoshkumar, P., Udupa, P., Murugesan, R., \& Sharma, K. K. (2008). Significance of interactions of low molecular weight crystallin fragments in lens aging and cataract formation. The Journal of biological chemistry, 283(13), 8477-8485.

Scarcelli, G., Besner, S., Pineda, R., Kalout, P., \& Yun, S. H. (2015). In Vivo Biomechanical Mapping of Normal and Keratoconus CorneasIn Vivo Biomechanical Mapping of CorneasLetters. JAMA Ophthalmology, 133(4), 480482.

Scarcelli, G., Kim, P., \& Yun, Seok H. (2011). In Vivo Measurement of Age-Related Stiffening in the Crystalline Lens by Brillouin Optical Microscopy. Biophysical Journal, 101(6), 1539-1545.

Scarcelli, G., Kling, S., Quijano, E., Pineda, R., Marcos, S., \& Yun, S. H. (2013). Brillouin microscopy of collagen crosslinking: noncontact depth-dependent analysis of corneal elastic modulus. Investigative Ophthalmology \& Visual Science, 54(2), 1418-1425.

Scarcelli, G., Pineda, R., \& Yun, S. H. (2012). Brillouin Optical Microscopy for Corneal Biomechanics. Investigative Ophthalmology \& Visual Science, 53(1), 185-190.

Scarcelli, G., \& Yun, S. H. (2007). Confocal Brillouin microscopy for threedimensional mechanical imaging. Nature Photonics, 2, 39.

Scarcelli, G., \& Yun, S. H. (2018). Reply to 'Water content, not stiffness, dominates Brillouin spectroscopy measurements in hydrated materials'. Nature Methods, 15(8), 562-563.

Seiler, T. G., Engler, M., Beck, E., Birngruber, R., \& Kochevar, I. (2017). Interface Bonding With Corneal Crosslinking (CXL) After LASIK Ex Vivo. Investigative Ophthalmology \& Visual Science, 58(14), 6292-6298.

Shao, P., Eltony, A., Seiler, T., Tavakol, B., Pineda, R., Koller, T., . . . Yun, S.-H. (2019). Spatially-resolved Brillouin spectroscopy reveals biomechanical abnormalities in mild to advanced keratoconus in vivo. Scientific Reports, 9(1), 7467.

Shao, P., Seiler, T. G., Eltony, A. M., Ramier, A., Kwok, S. J. J., Scarcelli, G., . . Yun, S.-H. (2018). Effects of Corneal Hydration on Brillouin Microscopy In VivoEffect of Corneal Hydration on Brillouin Frequency. Investigative Ophthalmology \& Visual Science, 59(7), 3020-3027.

Sheil, C. J., \& Goncharov, A. V. (2017). Crystalline lens paradoxes revisited: significance of age-related restructuring of the GRIN. Biomedical Optics Express, 8(9), 4172-4180.

Sheppard, A. L., Bashir, A., Wolffsohn, J. S., \& Davies, L. N. (2010). Accommodating intraocular lenses: a review of design concepts, usage and assessment methods. Clinical and Experimental Optometry, 93(6), 441-452.

Siezen, R. J., \& Owen, E. A. (1983). Physicochemical characterization of highmolecular-weight $\alpha$-crystallin subpopulations from the calf lens nucleus. Biochimica et Biophysica Acta (BBA) - Protein Structure and Molecular Enzymology, 749(3), 227-237.

Singh, M., Li, J., Han, Z., Wu, C., Aglyamov, S. R., Twa, M. D., \& Larin, K. V. (2016). Investigating Elastic Anisotropy of the Porcine Cornea as a Function of Intraocular Pressure With Optical Coherence Elastography. Journal of refractive surgery (Thorofare, N.J. : 1995), 32(8), 562-567.

Sinha Roy, A., Rocha, K. M., Randleman, J. B., Stulting, R. D., \& Dupps, W. J., Jr. (2013). Inverse computational analysis of in vivo corneal elastic modulus 
change after collagen crosslinking for keratoconus. Experimental Eye Research, 113, 92-104.

Stachs, O., Martin, H., Kirchhoff, A., Stave, J., Terwee, T., \& Guthoff, R. (2002). Monitoring accommodative ciliary muscle function using three-dimensional ultrasound. Graefe's Archive for Clinical and Experimental Ophthalmology, 240(11), 906-912.

Suzuki, S., Dawson, R. A., Chirila, T. V., Shadforth, A. M. A., Hogerheyde, T. A., Edwards, G. A., \& Harkin, D. G. (2015). Treatment of Silk Fibroin with Poly(ethylene glycol) for the Enhancement of Corneal Epithelial Cell Growth. Journal of functional biomaterials, 6(2), 345-366.

Tabernero, J., Chirre, E., Hervella, L., Prieto, P., \& Artal, P. (2016). The accommodative ciliary muscle function is preserved in older humans. Scientific Reports, 6, $25551 \mathrm{EP}$.

Taylor, V. L., al-Ghoul, K. J., Lane, C. W., Davis, V. A., Kuszak, J. R., \& Costello, M. J. (1996). Morphology of the normal human lens. Investigative Ophthalmology \& Visual Science, 37(7), 1396-1410.

Tomás-Juan, J., \& Murueta-Goyena, A. (2015). Axial movement of the dual-optic accommodating intraocular lens for the correction of the presbyopia: optical performance and clinical outcomes. Journal of Optometry, 8(2), 67-76.

Tranoudis, I., \& Efron, N. (2004). Tensile properties of soft contact lens materials. Contact Lens and Anterior Eye, 27(4), 177-191.

Tsao, S., Yao, M., Tsao, H., Henry, F. P., Zhao, Y., Kochevar, J. J., . . Kochevar, I. E. (2012). Light-activated tissue bonding for excisional wound closure: a splitlesion clinical trial. British Journal of Dermatology, 166(3), 555-563.

Turner, M. J., Clough, R. W., Martin, H. C., \& Topp, L. J. (1956). Stiffness and Deflection Analysis of Complex Structures. Journal of the Aeronautical Sciences, 23(9), 805-823.

Vellara, H. R., \& Patel, D. V. (2015). Biomechanical properties of the keratoconic cornea: a review. Clinical and Experimental Optometry, 98(1), 31-38.

Verter, E. E., Gisel, T. E., Yang, P., Johnson, A. J., Redmond, R. W., \& Kochevar, I. E. (2011). Light-Initiated Bonding of Amniotic Membrane to Cornea. Investigative Ophthalmology \& Visual Science, 52(13), 9470-9477.

Wang, K., Venetsanos, D., Wang, J., \& Pierscionek, B. K. (2016). Gradient moduli lens models: how material properties and application of forces can affect deformation and distributions of stress. Scientific Reports, 6, 31171.

Wang, S. B., Cornish, E. E., Grigg, J. R., \& McCluskey, P. J. (2019). Anterior segment optical coherence tomography and its clinical applications. Clinical and Experimental Optometry, 102(3), 195-207.

Weeber, H. A., Eckert, G., Pechhold, W., \& van der Heijde, R. G. L. (2007). Stiffness gradient in the crystalline lens. Graefe's Archive for Clinical and Experimental Ophthalmology, 245(9), 1357-1366.

Wilde, G. S., Burd, H. J., \& Judge, S. J. (2012). Shear modulus data for the human lens determined from a spinning lens test. Experimental Eye Research, 97(1), 36-48.

Wolbank, S., Hildner, F., Redl, H., van Griensven, M., Gabriel, C., \& Hennerbichler, S. (2009). Impact of human amniotic membrane preparation on release of angiogenic factors. Journal of Tissue Engineering and Regenerative Medicine, 3(8), 651-654.

Wollensak, G., Spoerl, E., \& Seiler, T. (2003). Stress-strain measurements of human and porcine corneas after riboflavin-ultraviolet-A-induced cross-linking. Journal of Cataract \& Refractive Surgery, 29(9), 1780-1785. 
Wu, P.-J., Kabakova, I., Ruberti, J., Sherwood, J., Dunlop, I. E., Paterson, C., . . . Overby, D. (2018). Water content, not stiffness, dominates Brillouin spectroscopy measurements in hydrated materials. Nature Methods, 15(8), 561562.

Zare Mehrjerdi, M. A., Mohebbi, M., \& Zandian, M. (2017). Review of Static Approaches to Surgical Correction of Presbyopia. Journal of ophthalmic \& vision research, 12(4), 413-418.

ZEMAX Optical Design Program User's Guide. (2005). In: Zemax Development Corporation.

Zhang, H., Qian, X., Li, L. I. N., \& Liu, Z. (2017). Understanding the viscoelastic properties of rabbit cornea based on stress relaxation tests and cyclic uniaxial tests. Journal of Mechanics in Medicine and Biology, 17(07), 1740035.

Zhao, H., Brown, P., Magone, M. T., \& Schuck, P. (2011). The Molecular Refractive Function of Lens $\gamma$-Crystallins. Journal of Molecular Biology, 411(3), 680-699.

Zhou, J., Zhang, B., Liu, X., Shi, L., Zhu, J., Wei, D., . . He, D. (2016). Facile method to prepare silk fibroin/hyaluronic acid films for vascular endothelial growth factor release. Carbohydrate Polymers, 143, 301-309.

Zienkiewicz, O. C., Taylor, R. L., \& Zhu, J. Z. (2005). The Finite Element Method. Oxford: Butterworth-Heinemann. 